\title{
Synthesis of quinoxaline derivatives via tandem oxidative azidation/cyclization reaction of $\mathrm{N}$-arylenamines
}

\author{
Haichao Ma, Dianjun Li, Wei Yu* \\ State Key Laboratory of Applied Organic Chemistry, College of \\ Chemistry and Chemical Engineering, Lanzhou University, Lanzhou, \\ Gansu 730000, P. R. China \\ yuwei@lzu.edu.cn
}

\section{Supporting Imformation}

\section{Contents}

Page

General methods

General procedure for the synthesis of $\mathbf{1}$

General procedure for the reactions of $\mathbf{1}$

Characterization data for substrates and products

S3-18

References

S18

Copies of ${ }^{1} \mathrm{H}$ NMR and ${ }^{13} \mathrm{C}$ NMR spectra of substrates

Copies of ${ }^{1} \mathrm{H}$ NMR and ${ }^{13} \mathrm{C}$ NMR spectra of products

S46-73

Single crystal X-ray diffraction data of $\mathbf{2 f}$

S74 


\section{General methods}

The ${ }^{1} \mathrm{H}$ and ${ }^{13} \mathrm{C}$ NMR spectra were recorded on an Agilent $\mathrm{AM}-300 \mathrm{MHz}$ spectrometer or a Bruker $\mathrm{AM}-400 \mathrm{MHz}$ spectrometer with $\mathrm{CDCl}_{3}$. The chemical shifts in ${ }^{1} \mathrm{H}$ NMR spectra were determined with $\mathrm{Si}\left(\mathrm{CH}_{3}\right)_{4}$ as the internal standard $(\delta=0.00$ ppm). The chemical shifts in ${ }^{13} \mathrm{C}$ NMR spectra were determined based on the chemical shift of $\mathrm{CDCl}_{3}(\delta=77.00 \mathrm{ppm})$. The high resolution mass spectra (HRMS) were measured on a Bruker micrOTOF QII by ESI. Melting points were measured on an XT-4 melting point apparatus and were uncorrected. Flash column chromatography was carried out on silica gel (200-300 mesh). Copric chloride $\left(\mathrm{CuCl}_{2}\right)$ was purchased from Aldrich. (Diacetoxyiodo)benzene $\left(\mathrm{PhI}(\mathrm{OAc})_{2}\right)$ and Azidotrimethylsilane $\left(\mathrm{TMSN}_{3}\right)$ was purchased from Energy Chemicals. Anhydrous acetonitrile and $N, N$-dimethylformamide (DMF) were purchased from Innochem Chemicals and used without further purification. Dichloromethane was distilled over $\mathrm{P}_{2} \mathrm{O}_{5}$ under an argon atmosphere before use.

\section{General procedure for the synthesis of 1}

Compounds (Z)-ethyl 3-aryl-3-(arylamino)acrylates (1a-1x), 3 and $\mathbf{4}$ were prepared according to the literature methods. ${ }^{1}$

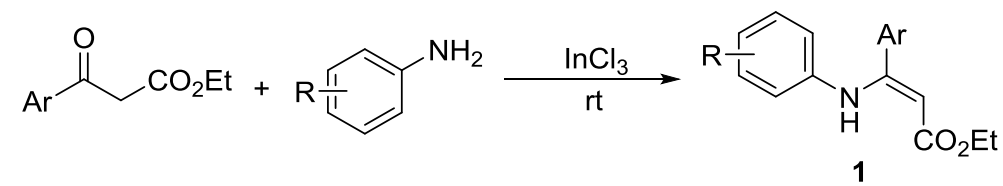

In a typical procedure, to a $10 \mathrm{~mL}$ round-bottom flask equipped with a magnetic stirring bar were added ethyl 3-oxo-3-arylpropanoate (5 mmol, 1.0 equiv), arylamine ( $5 \mathrm{mmol}, 1.0$ equiv) and Indium(III) chloride $\left(\mathrm{InCl}_{3}\right)(0.110 \mathrm{~g}, 0.5 \mathrm{mmol}, 0.1$ equiv). The mixture was stirred at room temperature. After the reaction was complete as indicated by TLC (generally 12-24 h), the reaction mixture was poured into $\mathrm{H}_{2} \mathrm{O}$ (25 $\mathrm{mL})$, and extracted with ethyl acetate $(10 \mathrm{~mL} \times 4)$. The combined organic layers were washed with brine $(30 \mathrm{~mL})$ and dried with anhydrous $\mathrm{Na}_{2} \mathrm{SO}_{4}$. The solvent was removed under reduced pressure with a rotatory evaporator, and the residual was treated with silica gel chromatography (using ethyl acetate/petroleum ether as the eluent) to give the $\mathbf{1}$.

Compounds 3-phenyl-3-phenylaminoacrylonitrile $(5)^{2}$ and $N$-(1-phenylethylidene)aniline $(\mathbf{6})^{3}$ were prepared according to the literature method.

\section{General procedure for the reactions of 1}




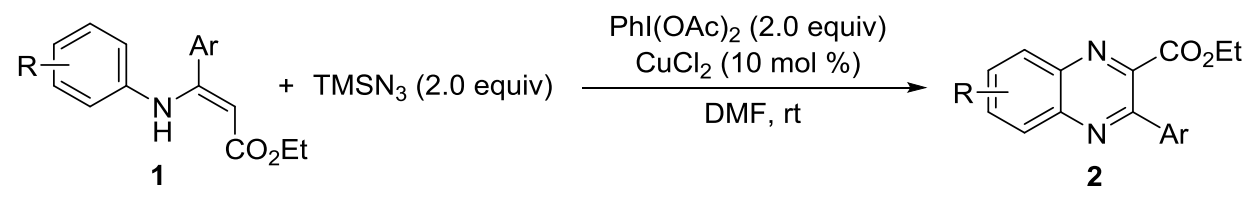

Into a $10 \mathrm{~mL}$ round-bottom flask equipped with a magnetic stirring bar were added sequentially 1 ( $0.5 \mathrm{mmol}, 1.0$ equiv), $\mathrm{CuCl}_{2}$ (6.7 mg, $0.05 \mathrm{mmol}, 0.1$ equiv), $\mathrm{TMSN}_{3}$ (131.5 $\mu \mathrm{L}, 1 \mathrm{mmol}, 2.0$ equiv), DMF (5 mL), and finally $\mathrm{PhI}(\mathrm{OAc})_{2}(128.8 \mathrm{mg}, 1$ mmol, 2.0 equiv). The mixture was stirred at room temperature. After the reaction was complete as indicated by TLC (generally 3-4 h), the reaction mixture was poured into a saturated aqueous $\mathrm{NaHSO}_{3}$ solution $(20 \mathrm{~mL})$, and was then extracted with ethyl acetate $(10 \mathrm{~mL} \times 4)$. The combined organic layers were washed with brine $(30 \mathrm{~mL} \times 4)$ and dried with anhydrous $\mathrm{Na}_{2} \mathrm{SO}_{4}$. The solvent was removed under reduced pressure, and the residual was treated with silica gel chromatography (using ethyl acetate/petroleum ether as the eluent) to give product(s) 2 .

The reation of $\mathbf{6}$ was conducted in acetonitrile in the absence of $\mathrm{CuCl}_{2}$ under the otherwise same conditions.

\section{Warning!}

Under the present reaction conditions of $\mathrm{TMSN}_{3} / \mathrm{PhI}(\mathrm{OAc})_{2} / \mathrm{CuCl}_{2}, \mathrm{HN}_{3}$ and $\mathrm{Cu}\left(\mathrm{N}_{3}\right)_{2}$ might be formed as the by-products. These compounds are toxic and explosive; the reactions must be handled carefully with sufficient protection.

\section{Spectroscopic data for the substrates}<smiles>CCOC(=O)C=C(Nc1ccccc1)OCC</smiles>

\section{(Z)-Ethyl 3-phenyl-3-(phenylamino)acrylate (1a) ${ }^{4}$}

${ }^{1} \mathrm{H}$ NMR $\left(\mathrm{CDCl}_{3}, 400 \mathrm{MHz}, \delta \mathrm{ppm}\right): 10.31(\mathrm{~s}, 1 \mathrm{H}), 7.35-7.33(\mathrm{~m}, 3 \mathrm{H}), 7.31-7.24(\mathrm{~m}$, $2 \mathrm{H}), 7.07(\mathrm{t}, J=8.0 \mathrm{~Hz}, 2 \mathrm{H}), 6.91-6.88(\mathrm{~m}, 1 \mathrm{H}), 6.66(\mathrm{~d}, J=7.6 \mathrm{~Hz}, 2 \mathrm{H}), 5.00(\mathrm{~s}$, $1 \mathrm{H}), 4.20(\mathrm{q}, J=7.2 \mathrm{~Hz}, 2 \mathrm{H}), 1.31(\mathrm{t}, J=7.2 \mathrm{~Hz}, 3 \mathrm{H}) ;{ }^{13} \mathrm{C} \mathrm{NMR}\left(\mathrm{CDCl}_{3}, 75 \mathrm{MHz}, \delta\right.$ ppm): 170.0, 159.0, 140.3, 135.8, 129.4, 128.5, 128.4, 128.1, 122.9, 122.1, 91.1, 59.3, 14.5 .<smiles>CCOC(=O)/C=C(\Nc1ccc(C)cc1)c1ccccc1</smiles>

(Z)-Ethyl 3-phenyl-3-(p-tolylamino)acrylate (1b) ${ }^{4}$

${ }^{1} \mathrm{H}$ NMR $\left(\mathrm{CDCl}_{3}, 400 \mathrm{MHz}, \delta \mathrm{ppm}\right): 10.26(\mathrm{~s}, 1 \mathrm{H}), 7.33-7.23(\mathrm{~m}, 5 \mathrm{H}), 6.86(\mathrm{~d}, J=$ $8.0 \mathrm{~Hz}, 2 \mathrm{H}), 6.56$ (d, $J=8.4 \mathrm{~Hz}, 2 \mathrm{H}), 4.95$ (s, 1H), 4.20 (q, J=7.2 Hz, 2H), 2.19 (s, $3 \mathrm{H}), 1.31(\mathrm{t}, J=7.2 \mathrm{~Hz}, 3 \mathrm{H}) ;{ }^{13} \mathrm{C} \mathrm{NMR}\left(\mathrm{CDCl}_{3}, 75 \mathrm{MHz}, \delta \mathrm{ppm}\right): 170.1,159.3,137.7$, 
$136.0,132.5,129.3,129.1,128.3,128.2,122.3,90.2,59.2,20.7,14.5$.<smiles>CCOC(=Cc1ccccc1)Nc1ccc(OC)cc1</smiles>

\section{(Z)-Ethyl 3-((4-methoxyphenyl)amino)-3-phenylacrylate (1c) ${ }^{4}$}

${ }^{1} \mathrm{H}$ NMR $\left(\mathrm{CDCl}_{3}, 400 \mathrm{MHz}, \delta \mathrm{ppm}\right): 10.23$ (s, 1H), 7.32-7.23 (m, 5H), 6.63 (s, 4H), $4.93(\mathrm{~s}, 1 \mathrm{H}), 4.20(\mathrm{q}, J=7.2 \mathrm{~Hz}, 2 \mathrm{H}), 3.68(\mathrm{~s}, 3 \mathrm{H}), 1.31(\mathrm{t}, J=7.2 \mathrm{~Hz}, 3 \mathrm{H}) ;{ }^{13} \mathrm{C} \mathrm{NMR}$ $\left(\mathrm{CDCl}_{3}, 75 \mathrm{MHz}, \delta \mathrm{ppm}\right): 170.2,159.8,155.7,135.9,133.4,129.2,128.3,128.2$, $124.2,113.8,89.4,59.1,55.2,14.5$.<smiles>CCOC(=O)C=C(OCC)c1ccccc1</smiles>

\section{(Z)-Ethyl 3-((4-fluorophenyl)amino)-3-phenylacrylate (1d $)^{5}$}

${ }^{1} \mathrm{H}$ NMR $\left(\mathrm{CDCl}_{3}, 400 \mathrm{MHz}, \delta \mathrm{ppm}\right): 10.25$ (s, 1H), 7.35-7.25 (m, 5H), 6.80-6.74 (m, $2 \mathrm{H}), 6.65-6.62(\mathrm{~m}, 2 \mathrm{H}), 5.00(\mathrm{~s}, 1 \mathrm{H}), 4.20(\mathrm{q}, J=7.2 \mathrm{~Hz}, 2 \mathrm{H}), 1.31(\mathrm{t}, J=7.2 \mathrm{~Hz}$, $3 \mathrm{H}) ;{ }^{13} \mathrm{C} \mathrm{NMR}\left(\mathrm{CDCl}_{3}, 100 \mathrm{MHz}, \delta \mathrm{ppm}\right): 170.1,159.2,158.8(\mathrm{~d}, J=241 \mathrm{~Hz}), 136.4$, 136.4, 135.6, 129.4, 128.4, 128.2, 124.0, 123.9, 115.4, 115.2, 90.8, 59.3, 14.5.<smiles>CCOC(=O)/C=C(\Nc1ccc(Cl)cc1)c1ccccc1</smiles>

\section{(Z)-Ethyl 3-((4-chlorophenyl)amino)-3-phenylacrylate (1e) ${ }^{4}$}

${ }^{1} \mathrm{H}$ NMR $\left(\mathrm{CDCl}_{3}, 400 \mathrm{MHz}, \delta \mathrm{ppm}\right): 10.28(\mathrm{~s}, 1 \mathrm{H}), 7.37-7.25(\mathrm{~m}, 5 \mathrm{H}), 7.04-7.01$ (m, 2H), 6.59-6.55 (m, 2H), $5.02(\mathrm{~s}, 1 \mathrm{H}), 4.20(\mathrm{q}, J=7.2 \mathrm{~Hz}, 2 \mathrm{H}), 1.31(\mathrm{t}, J=7.2 \mathrm{~Hz}$, $3 \mathrm{H}) ;{ }^{13} \mathrm{C} \mathrm{NMR}\left(\mathrm{CDCl}_{3}, 75 \mathrm{MHz}, \delta \mathrm{ppm}\right): 170.0,158.5,138.9,135.4,129.6,128.6$, $128.5,128.1,123.1,91.8,59.4,14.4$.<smiles>CCOC(=O)/C=C(\Nc1ccc(Br)cc1)c1ccccc1</smiles>

(Z)-Ethyl 3-((4-bromophenyl)amino)-3-phenylacrylate (1f $)^{4}$

${ }^{1} \mathrm{H}$ NMR $\left(\mathrm{CDCl}_{3}, 400 \mathrm{MHz}, \delta \mathrm{ppm}\right): 10.27(\mathrm{~s}, 1 \mathrm{H}), 7.36-7.26(\mathrm{~m}, 5 \mathrm{H}), 7.18-7.15(\mathrm{~m}$, $2 \mathrm{H}), 6.51(\mathrm{~d}, J=8.8 \mathrm{~Hz}, 2 \mathrm{H}), 5.03(\mathrm{~s}, 1 \mathrm{H}), 4.20(\mathrm{q}, J=7.2 \mathrm{~Hz}, 2 \mathrm{H}), 1.31(\mathrm{t}, J=7.2$ $\mathrm{Hz}, 3 \mathrm{H}) ;{ }^{13} \mathrm{C} \mathrm{NMR}\left(\mathrm{CDCl}_{3}, 75 \mathrm{MHz}, \delta \mathrm{ppm}\right): 170.0,158.4,139.5,135.4,131.5,129.6$, $128.5,128.1,123.4,115.6,92.0,59.4,14.5$.<smiles>CCOC(=CC(Nc1ccc(I)cc1)c1ccccc1)OCC</smiles> 


\section{(Z)-Ethyl 3-((4-iodophenyl)amino)-3-phenylacrylate (1g) ${ }^{4}$}

${ }^{1} \mathrm{H} \mathrm{NMR}\left(\mathrm{CDCl}_{3}, 400 \mathrm{MHz}, \delta \mathrm{ppm}\right): 10.27$ (s, 1H), 7.38-7.26 (m, 7H), 6.39 (d, $J=$ $8.4 \mathrm{~Hz}, 2 \mathrm{H}), 5.03(\mathrm{~s}, 1 \mathrm{H}), 4.20(\mathrm{q}, J=7.2 \mathrm{~Hz}, 2 \mathrm{H}), 1.31(\mathrm{t}, J=7.2 \mathrm{~Hz}, 3 \mathrm{H}) ;{ }^{13} \mathrm{C} \mathrm{NMR}$ $\left(\mathrm{CDCl}_{3}, 100 \mathrm{MHz}, \delta \mathrm{ppm}\right): 170.0,158.3,140.2,137.5,135.5,129.6,128.6,128.1$, 123.7, 92.2, 86.0, 59.5, 14.5.<smiles>CCOC(=O)C=C(Nc1ccc(C(F)(F)F)cc1)OCC</smiles>

\section{(Z)-Ethyl 3-phenyl-3-((4-(trifluoromethyl)phenyl)amino)acrylate (1h) ${ }^{4}$}

${ }^{1} \mathrm{H} \mathrm{NMR}\left(\mathrm{CDCl}_{3}, 400 \mathrm{MHz}, \delta \mathrm{ppm}\right): 10.40(\mathrm{~s}, 1 \mathrm{H}), 7.42-7.26(\mathrm{~m}, 7 \mathrm{H}), 6.67(\mathrm{~d}, J=$ $8.4 \mathrm{~Hz}, 2 \mathrm{H}), 5.11(\mathrm{~s}, 1 \mathrm{H}), 4.22(\mathrm{q}, J=7.2 \mathrm{~Hz}, 2 \mathrm{H}), 1.32(\mathrm{t}, J=7.2 \mathrm{~Hz}, 3 \mathrm{H}) ;{ }^{13} \mathrm{C} \mathrm{NMR}$ $\left(\mathrm{CDCl}_{3}, 100 \mathrm{MHz}, \delta \mathrm{ppm}\right): 169.9,157.7,143.6,135.3,129.9,128.7,128.2,128.0$, 125.9, 125.9, 125.8, 125.8, 125.5, 124.0 (q, $J=32 \mathrm{~Hz}), 122.8,120.8,120.1,93.7,59.6$, 14.4 .<smiles>CCOC(=O)/C=C(\Nc1ccccc1)c1ccccc1</smiles>

\section{(Z)-Ethyl 3-([1,1'-biphenyl]-4-ylamino)-3-phenylacrylate (1i)}

Pale yellow solid; m.p. $=114-115{ }^{\circ} \mathrm{C} ;{ }^{1} \mathrm{H}$ NMR $\left(\mathrm{CDCl}_{3}, 300 \mathrm{MHz}, \delta\right.$ ppm): 10.38 (s, 1H), 7.50-7.47 (m, 2H), 7.40-7.25 (m, 10H), $6.71(\mathrm{~d}, J=8.4 \mathrm{~Hz}, 2 \mathrm{H}), 5.02(\mathrm{~s}, 1 \mathrm{H})$, $4.22(\mathrm{q}, J=7.2 \mathrm{~Hz}, 2 \mathrm{H}), 1.33(\mathrm{t}, J=7.2 \mathrm{~Hz}, 3 \mathrm{H}) ;{ }^{13} \mathrm{C} \mathrm{NMR}\left(\mathrm{CDCl}_{3}, 75 \mathrm{MHz}, \delta \mathrm{ppm}\right)$ : $170.1,158.7,140.3,139.6,135.9$, 135.4, 129.5, 128.7, 128.5, 128.2, 127.1, 126.9, 126.6, 122.1, 91.4, 59.3, 14.5; HRMS (ESI): calcd. for $\mathrm{C}_{23} \mathrm{H}_{21} \mathrm{~N}_{1} \mathrm{O}_{2}+\mathrm{H}=344.1645$, found: 344.1649 .<smiles>CCOC(=O)C=C(Nc1cccc(C)c1)OCC</smiles>

\section{(Z)-Ethyl 3-phenyl-3-(m-tolylamino)acrylate (1j) $)^{4}$}

${ }^{1} \mathrm{H} \mathrm{NMR}\left(\mathrm{CDCl}_{3}, 400 \mathrm{MHz}, \delta \mathrm{ppm}\right): 10.28(\mathrm{~s}, 1 \mathrm{H}), 7.35-7.31(\mathrm{~m}, 3 \mathrm{H}), 7.29-7.24(\mathrm{~m}$, 2H), $6.92(\mathrm{t}, J=8.0 \mathrm{~Hz}, 1 \mathrm{H}), 6.71(\mathrm{~d}, J=7.2 \mathrm{~Hz}, 1 \mathrm{H}), 6.54(\mathrm{~s}, 1 \mathrm{H}), 6.38(\mathrm{~d}, J=8.0$ $\mathrm{Hz}, 1 \mathrm{H}), 4.98(\mathrm{~s}, 1 \mathrm{H}), 4.20(\mathrm{q}, J=7.2 \mathrm{~Hz}, 2 \mathrm{H}), 2.16(\mathrm{~s}, 1 \mathrm{H}), 1.31(\mathrm{t}, J=7.2 \mathrm{~Hz}, 3 \mathrm{H})$; ${ }^{13} \mathrm{C} \mathrm{NMR}\left(\mathrm{CDCl}_{3}, 75 \mathrm{MHz}, \delta \mathrm{ppm}\right): 170.0,159.0,140.2,138.4,136.0,129.3,128.3$, $128.2,128.1,123.6,122.7,119.2,90.8,59.2,21.3,14.5$.<smiles>CCOC(=O)C=C(OCC)c1cccc(Cl)c1</smiles>

(Z)-Ethyl 3-((3-chlorophenyl)amino)-3-phenylacrylate (1k) 
Pale yellow solid; m.p. $=52-54{ }^{\circ} \mathrm{C} ;{ }^{1} \mathrm{H} \mathrm{NMR}\left(\mathrm{CDCl}_{3}, 300 \mathrm{MHz}, \delta \mathrm{ppm}\right): 10.28(\mathrm{~s}$, $1 \mathrm{H}), 7.40-7.26(\mathrm{~m}, 5 \mathrm{H}), 6.97(\mathrm{t}, J=8.1 \mathrm{~Hz}, 1 \mathrm{H}), 6.86(\mathrm{~d}, J=7.5 \mathrm{~Hz}, 1 \mathrm{H}), 6.67$ (s, $1 \mathrm{H}), 6.48(\mathrm{~d}, J=8.1 \mathrm{~Hz}, 1 \mathrm{H}), 5.05(\mathrm{~s}, 1 \mathrm{H}), 4.21$ (q, $J=7.2 \mathrm{~Hz}, 2 \mathrm{H}), 1.34-1.29$ (m, $3 \mathrm{H}) ;{ }^{13} \mathrm{C} \mathrm{NMR}\left(\mathrm{CDCl}_{3}, 75 \mathrm{MHz}, \delta \mathrm{ppm}\right): 169.9,158.2,141.7,135.4,134.1,129.7$, 129.4, 128.5, 128.0, 122.7, 121.7, 119.9, 92.5, 59.5, 14.4; HRMS (ESI): calcd. for $\mathrm{C}_{17} \mathrm{H}_{16} \mathrm{Cl}_{1} \mathrm{~N}_{1} \mathrm{O}_{2}+\mathrm{H}=302.0942$, found: 302.0947 .<smiles>CCOC(=O)/C=C(\Nc1ccccc1[N+](C)(C)C)c1ccccc1</smiles>

(Z)-Ethyl 3-phenyl-3-(o-tolylamino)acrylate (11) ${ }^{4}$

${ }^{1} \mathrm{H}$ NMR $\left(\mathrm{CDCl}_{3}, 400 \mathrm{MHz}, \delta \mathrm{ppm}\right): 10.16$ (s, 1H), 7.29-7.22 (m, 5H), 7.12 (d, $J=$ $7.2 \mathrm{~Hz}, 1 \mathrm{H}), 6.86-6.82(\mathrm{~m}, 1 \mathrm{H}), 6.79-6.75(\mathrm{~m}, 1 \mathrm{H}), 6.32(\mathrm{~d}, J=7.6 \mathrm{~Hz}, 1 \mathrm{H}), 5.04$ (s, $1 \mathrm{H}), 4.21(\mathrm{q}, J=7.2 \mathrm{~Hz}, 2 \mathrm{H}), 2.42(\mathrm{~s}, 1 \mathrm{H}), 1.31(\mathrm{t}, J=7.2 \mathrm{~Hz}, 3 \mathrm{H}) ;{ }^{13} \mathrm{C} \mathrm{NMR}\left(\mathrm{CDCl}_{3}\right.$, $75 \mathrm{MHz}, \delta \mathrm{ppm}): 170.2$, 159.7, 138.9, 136.1, 130.3, 130.1, 129.3, 128.3, 128.0, 125.7, $123.7,123.4,90.7,59.2,18.2,14.5$.<smiles>CCOC(=O)/C=C(\Nc1ccccc1OC)c1ccccc1</smiles>

\section{(Z)-Ethyl 3-((2-methoxyphenyl)amino)-3-phenylacrylate (1m)}

${ }^{1} \mathrm{H} \mathrm{NMR}\left(\mathrm{CDCl}_{3}, 400 \mathrm{MHz}, \delta \mathrm{ppm}\right): 10.29$ (s, 1H), 7.37-7.25 (m, 5H), 6.87-6.81 (m, $2 \mathrm{H}), 6.50(\mathrm{t}, J=7.2 \mathrm{~Hz}, 1 \mathrm{H}), 6.20(\mathrm{~d}, J=8.0 \mathrm{~Hz}, 1 \mathrm{H}), 5.00(\mathrm{~s}, 1 \mathrm{H}), 4.22(\mathrm{q}, J=7.2$ $\mathrm{Hz}, 2 \mathrm{H}), 3.89(\mathrm{~s}, 1 \mathrm{H}), 1.31(\mathrm{t}, J=7.2 \mathrm{~Hz}, 3 \mathrm{H}) ;{ }^{13} \mathrm{C} \mathrm{NMR}\left(\mathrm{CDCl}_{3}, 75 \mathrm{MHz}, \delta\right.$ ppm):169.8, 158.3, 150.3, 136.2, 129.5, 129.3, 128.3, 127.9, 122.8, 121.7, 119.8, $110.4,91.5,59.2,55.6,14.5$.<smiles>CCOC(=CC(=Cc1ccccc1)Nc1ccccc1Cl)OCC</smiles>

\section{(Z)-Ethyl 3-((2-chlorophenyl)amino)-3-phenylacrylate (1n)}

Yellow solid; m.p. $=76-78{ }^{\circ} \mathrm{C} ;{ }^{1} \mathrm{H} \mathrm{NMR}\left(\mathrm{CDCl}_{3}, 300 \mathrm{MHz}, \delta \mathrm{ppm}\right): 10.35(\mathrm{~s}, 1 \mathrm{H})$, 7.35-7.26 (m, 6H), 6.83-6.79 (m, 2H), 6.30-6.27 (m, 1H), $5.12(\mathrm{~s}, 1 \mathrm{H}), 4.24$ (q, $J=$ $7.2 \mathrm{~Hz}, 2 \mathrm{H}), 1.32$ (t, $J=7.2 \mathrm{~Hz}, 3 \mathrm{H}) ;{ }^{13} \mathrm{C} \mathrm{NMR}\left(\mathrm{CDCl}_{3}, 75 \mathrm{MHz}, \delta \mathrm{ppm}\right): 169.7$, $157.7,137.5,135.7,129.6,129.5,128.5,127.9,126.3,125.6,123.3,123.3,93.5,59.5$, 14.5; HRMS (ESI): calcd. for $\mathrm{C}_{17} \mathrm{H}_{16} \mathrm{Cl}_{1} \mathrm{~N}_{1} \mathrm{O}_{2}+\mathrm{H}=302.0942$, found: 302.0945 .<smiles>CCOC(=CC(=Cc1ccccc1)Nc1ccccc1Br)OCC</smiles>

(Z)-Ethyl 3-((2-bromophenyl)amino)-3-phenylacrylate (10) ${ }^{6}$ 
${ }^{1} \mathrm{H} \mathrm{NMR}\left(\mathrm{CDCl}_{3}, 400 \mathrm{MHz}, \delta \mathrm{ppm}\right): 10.29(\mathrm{~s}, 1 \mathrm{H}), 7.52(\mathrm{dd}, J=8.0 \mathrm{~Hz}, 1.2 \mathrm{~Hz}, 1 \mathrm{H})$, 7.36-7.25 (m, 5H), 6.85-6.81 (m, 1H), 6.77-6.73 (m, 1H), 6.29 (dd, $J=8.0 \mathrm{~Hz}, 1.2$ $\mathrm{Hz}, 1 \mathrm{H}), 5.13(\mathrm{~s}, 1 \mathrm{H}), 4.24(\mathrm{q}, J=7.2 \mathrm{~Hz}, 2 \mathrm{H}), 1.32(\mathrm{t}, J=7.2 \mathrm{~Hz}, 3 \mathrm{H}) ;{ }^{13} \mathrm{C} \mathrm{NMR}$ $\left(\mathrm{CDCl}_{3}, 100 \mathrm{MHz}, \delta \mathrm{ppm}\right): 169.7,157.7,138.8,135.6,132.7,129.6,128.5,127.9$, $127.0,123.8,123.7,116.1,93.4,59.5,14.5$.<smiles>CCOC(=Cc1ccccc1)Nc1ccccc1C(F)(F)F</smiles>

\section{(Z)-Ethyl 3-phenyl-3-((2-(trifluoromethyl)phenyl)amino)acrylate (1p)}

White solid; m.p. $=131-133{ }^{\circ} \mathrm{C} ;{ }^{1} \mathrm{H}$ NMR $\left(\mathrm{CDCl}_{3}, 400 \mathrm{MHz}, \delta \mathrm{ppm}\right): 10.40(\mathrm{~s}, 1 \mathrm{H})$, $7.58(\mathrm{~d}, J=7.2 \mathrm{~Hz}, 1 \mathrm{H}), 7.34-7.30(\mathrm{~m}, 3 \mathrm{H}), 7.28-7.24(\mathrm{~m}, 2 \mathrm{H}), 7.07$ (t, $J=7.6 \mathrm{~Hz}$, $1 \mathrm{H}), 6.99$ (t, $J=7.6 \mathrm{~Hz}, 1 \mathrm{H}), 6.43(\mathrm{~d}, J=8.0 \mathrm{~Hz}, 1 \mathrm{H}), 5.19$ (s, 1H), 4.24 (q, $J=7.2$ $\mathrm{Hz}, 2 \mathrm{H}), 1.32(\mathrm{t}, J=7.2 \mathrm{~Hz}, 3 \mathrm{H}) ;{ }^{13} \mathrm{C} \mathrm{NMR}\left(\mathrm{CDCl}_{3}, 100 \mathrm{MHz}, \delta \mathrm{ppm}\right): 169.7,158.1$, 139.0, 135.7, 131.6, 129.6, 128.6, 128.1, 126.3, 126.3, 126.2, 126.2, 125.8, 125.5, 122.8, $122.0(\mathrm{~d}, J=29 \mathrm{~Hz}), 94.3,59.6,14.4$; HRMS (ESI): calcd. for $\mathrm{C}_{18} \mathrm{H}_{16} \mathrm{~F}_{3} \mathrm{~N}_{1} \mathrm{O}_{2}+$ $\mathrm{H}=336.1206$, found: 336.1209 .<smiles>CCOC(=O)/C=C(\Nc1cc(C)cc([N+](=O)[O-])c1)c1ccccc1</smiles>

\section{(Z)-Ethyl 3-((3,5-dimethylphenyl)amino)-3-phenylacrylate (1q)}

White solid; m.p. $=67-69{ }^{\circ} \mathrm{C} ;{ }^{1} \mathrm{H}$ NMR $\left(\mathrm{CDCl}_{3}, 400 \mathrm{MHz}, \delta \mathrm{ppm}\right): 10.25(\mathrm{~s}, 1 \mathrm{H})$, 7.35-7.25 (m, 5H), $6.54(\mathrm{~s}, 1 \mathrm{H}), 6.26(\mathrm{~s}, 2 \mathrm{H}), 4.96(\mathrm{~s}, 1 \mathrm{H}), 4.20(\mathrm{q}, J=7.2 \mathrm{~Hz}, 2 \mathrm{H})$, $2.09(\mathrm{~s}, 6 \mathrm{H}), 1.31(\mathrm{t}, J=7.2 \mathrm{~Hz}, 3 \mathrm{H}) ;{ }^{13} \mathrm{C} \mathrm{NMR}\left(\mathrm{CDCl}_{3}, 100 \mathrm{MHz}, \delta \mathrm{ppm}\right): 170.1$, 159.2, 140.1, 138.1, 136.1, 129.3, 128.3, 128.1, 124.6, 119.9, 90.6, 59.2, 21.2, 14.5; HRMS (ESI): calcd. for $\mathrm{C}_{19} \mathrm{H}_{21} \mathrm{~N}_{1} \mathrm{O}_{2}+\mathrm{H}=296.1645$, found: 296.1649 .<smiles>CCOC(=O)C=C(Nc1cc(C(F)(F)F)cc(C(F)(F)F)c1)OCC</smiles>

\section{(Z)-Ethyl 3-((3,5-bis(trifluoromethyl)phenyl)amino)-3-phenylacrylate (1r)}

Pale yellow solid; m.p. $=77-78{ }^{\circ} \mathrm{C} ;{ }^{1} \mathrm{H}$ NMR $\left(\mathrm{CDCl}_{3}, 400 \mathrm{MHz}, \delta \mathrm{ppm}\right): 10.55$ (s, 1H), 7.44-7.43 (m, 1H), 7.42-7.33 (m, 5H), 6.97 (s, 2H), 5.19 (s, 1H), 4.24 (q, $J=$ $7.2 \mathrm{~Hz}, 2 \mathrm{H}), 1.33$ (t, $J=7.2 \mathrm{~Hz}, 3 \mathrm{H}) ;{ }^{13} \mathrm{C} \mathrm{NMR}\left(\mathrm{CDCl}_{3}, 100 \mathrm{MHz}, \delta \mathrm{ppm}\right): 169.8$, 157.2, 142.0, 134.5, 131.9 (q, $J=33 \mathrm{~Hz}$ ), 130.2, 129.0, 127.9, 127.0, 124.3, 121.5, 120.6, 118.8, 115.4, 115.4, 115.3, 94.7, 59.9, 14.4; HRMS (ESI): calcd. for $\mathrm{C}_{19} \mathrm{H}_{15} \mathrm{~F}_{6} \mathrm{~N}_{1} \mathrm{O}_{2}+\mathrm{H}=404.1080$, found: 404.1084 . 


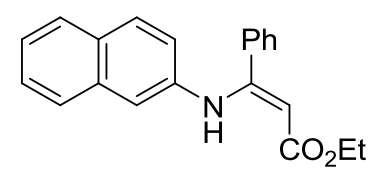

(Z)-Ethyl 3-(naphthalen-2-ylamino)-3-phenylacrylate (1s)

Yellow solid; m.p. $=80-82{ }^{\circ} \mathrm{C} ;{ }^{1} \mathrm{H}$ NMR $\left(\mathrm{CDCl}_{3}, 400 \mathrm{MHz}, \delta \mathrm{ppm}\right): 10.52(\mathrm{~s}, 1 \mathrm{H})$, $7.63(\mathrm{~d}, J=7.6 \mathrm{~Hz}, 1 \mathrm{H}), 7.51(\mathrm{~d}, J=8.4 \mathrm{~Hz}, 1 \mathrm{H}), 7.46(\mathrm{~d}, J=8.0 \mathrm{~Hz}, 1 \mathrm{H}), 7.38-7.36$ (m, 2H), 7.33-7.19 (m, 5H), $7.00(\mathrm{~s}, 1 \mathrm{H}), 6.84(\mathrm{dd}, J=8.8 \mathrm{~Hz}, 2.4 \mathrm{~Hz}, 1 \mathrm{H}), 5.06$ (s, $1 \mathrm{H}), 4.22(\mathrm{q}, J=7.2 \mathrm{~Hz}, 2 \mathrm{H}), 1.31(\mathrm{t}, J=7.2 \mathrm{~Hz}, 3 \mathrm{H}) ;{ }^{13} \mathrm{C} \mathrm{NMR}\left(\mathrm{CDCl}_{3}, 75 \mathrm{MHz}, \delta\right.$ ppm): 170.1, 158.9, 137.9, 135.9, 133.6, 129.8, 129.5, 128.5, 128.3, 128.2, 127.5, 127.0, 126.3, 124.5, 122.4, 118.3, 91.5, 59.4, 14.5; HRMS (ESI): calcd. for $\mathrm{C}_{21} \mathrm{H}_{19} \mathrm{~N}_{1} \mathrm{O}_{2}+\mathrm{H}=318.1489$, found: 318.1495 .<smiles>CCOC(=O)/C=C(\Nc1cccc2ccccc12)c1ccccc1</smiles>

\section{(Z)-Ethyl 3-(naphthalen-1-ylamino)-3-phenylacrylate (1t) ${ }^{4}$}

${ }^{1} \mathrm{H}$ NMR $\left(\mathrm{CDCl}_{3}, 400 \mathrm{MHz}, \delta \mathrm{ppm}\right): 10.70(\mathrm{~s}, 1 \mathrm{H}), 8.33$ (d, J = 8.4 Hz, 1H), $7.80(\mathrm{~d}$, $J=8.0 \mathrm{~Hz}, 1 \mathrm{H}), 7.59-7.56(\mathrm{~m}, 1 \mathrm{H}), 7.52-7.45(\mathrm{~m}, 2 \mathrm{H}), 7.30(\mathrm{~d}, J=7.2 \mathrm{~Hz}, 2 \mathrm{H}), 7.25$ $(\mathrm{t}, J=7.2 \mathrm{~Hz}, 1 \mathrm{H}), 7.18-7.15(\mathrm{~m}, 2 \mathrm{H}), 7.06-7.02(\mathrm{~m}, 1 \mathrm{H}), 6.49(\mathrm{~d}, J=7.6 \mathrm{~Hz}, 1 \mathrm{H})$, $5.17(\mathrm{~s}, 1 \mathrm{H}), 4.25(\mathrm{q}, J=7.2 \mathrm{~Hz}, 2 \mathrm{H}), 1.34(\mathrm{t}, J=7.2 \mathrm{~Hz}, 3 \mathrm{H}) ;{ }^{13} \mathrm{C} \mathrm{NMR}\left(\mathrm{CDCl}_{3}, 100\right.$ $\mathrm{MHz}, \delta \mathrm{ppm}): 170.4,160.3,136.2,136.0,134.0,129.3,128.3,127.9,126.3,126.2$, $125.0,123.9,122.0,121.1,91.7,59.4,14.5$.<smiles>CCOC(=O)/C=C(/Nc1ccccc1)c1ccc(OC)cc1</smiles>

\section{(Z)-Ethyl 3-(4-methoxyphenyl)-3-(phenylamino)acrylate $(1 \mathbf{u})^{7}$}

${ }^{1} \mathrm{H}$ NMR $\left(\mathrm{CDCl}_{3}, 400 \mathrm{MHz}, \delta \mathrm{ppm}\right): 10.27(\mathrm{~s}, 1 \mathrm{H}), 7.29-7.25(\mathrm{~m}, 2 \mathrm{H}), 7.08$ (t, $J=$ $8.0 \mathrm{~Hz}, 2 \mathrm{H}), 6.92-6.88(\mathrm{~m}, 1 \mathrm{H}), 6.80-6.78(\mathrm{~m}, 2 \mathrm{H}), 6.68(\mathrm{~d}, J=8.0 \mathrm{~Hz}, 2 \mathrm{H}), 4.97$ (s, $1 \mathrm{H}), 4.19(\mathrm{q}, J=7.2 \mathrm{~Hz}, 2 \mathrm{H}), 3.77(\mathrm{~s}, 3 \mathrm{H}), 1.31(\mathrm{t}, J=7.2 \mathrm{~Hz}, 3 \mathrm{H}) ;{ }^{13} \mathrm{C} \mathrm{NMR}\left(\mathrm{CDCl}_{3}\right.$, $100 \mathrm{MHz}, \delta \mathrm{ppm}): 170.1,160.4,158.7,140.5,129.6,128.5,128.0,122.7,122.1$, $113.7,90.4,59.2,55.2,14.5$.<smiles>CCOC(=O)/C=C(/Nc1ccccc1)c1ccc(Br)cc1</smiles> 


\section{(Z)-Ethyl 3-(4-bromophenyl)-3-(phenylamino)acrylate (1v) ${ }^{8}$}

${ }^{1} \mathrm{H}$ NMR $\left(\mathrm{CDCl}_{3}, 300 \mathrm{MHz}, \delta \mathrm{ppm}\right): 10.25$ (s, 1H), $7.41(\mathrm{~d}, J=8.4 \mathrm{~Hz}, 2 \mathrm{H})$, 7.23-7.20 (m, 2H), $7.10(\mathrm{t}, J=7.8 \mathrm{~Hz}, 2 \mathrm{H}), 6.93(\mathrm{t}, J=7.5 \mathrm{~Hz}, 1 \mathrm{H}), 6.66(\mathrm{~d}, J=7.8$ $\mathrm{Hz}, 2 \mathrm{H}), 4.97(\mathrm{~s}, 1 \mathrm{H}), 4.20(\mathrm{q}, J=7.2 \mathrm{~Hz}, 2 \mathrm{H}), 1.31(\mathrm{t}, J=7.2 \mathrm{~Hz}, 3 \mathrm{H}) ;{ }^{13} \mathrm{C} \mathrm{NMR}$ $\left(\mathrm{CDCl}_{3}, 75 \mathrm{MHz}, \delta \mathrm{ppm}\right): 169.9,157.6,140.0,134.8,131.6,129.7,128.7,123.7$, $123.2,122.3,91.4,59.4,14.5$.<smiles>CCOC(=O)/C=C(/Nc1ccccc1)c1ccccn1</smiles>

\section{(Z)-Ethyl 3-(phenylamino)-3-(pyridin-2-yl)acrylate (1w)}

Yellow solid; m.p. $=58-59{ }^{\circ} \mathrm{C} ;{ }^{1} \mathrm{H}$ NMR $\left(\mathrm{CDCl}_{3}, 400 \mathrm{MHz}, \delta \mathrm{ppm}\right): 10.16(\mathrm{~s}, 1 \mathrm{H})$, 8.60-8.59 (m, 1H), 7.57 (t, $J=7.6 \mathrm{~Hz}, 1 \mathrm{H}), 7.31-7.28(\mathrm{~m}, 1 \mathrm{H}), 7.25-7.22(\mathrm{~m}, 1 \mathrm{H})$, $7.08(\mathrm{t}, J=7.2 \mathrm{~Hz}, 2 \mathrm{H}), 6.91(\mathrm{t}, J=7.2 \mathrm{~Hz}, 1 \mathrm{H}), 6.66(\mathrm{~d}, J=8.0 \mathrm{~Hz}, 2 \mathrm{H}), 5.32(\mathrm{~s}, 1 \mathrm{H})$, $4.22(\mathrm{q}, J=7.2 \mathrm{~Hz}, 2 \mathrm{H}), 1.30(\mathrm{t}, J=7.2 \mathrm{~Hz}, 3 \mathrm{H}) ;{ }^{13} \mathrm{C} \mathrm{NMR}\left(\mathrm{CDCl}_{3}, 100 \mathrm{MHz}, \delta\right.$ ppm): 170.0, 156.7, 154.0, 149.6, 140.4, 136.2, 128.6, 123.8, 123.6, 122.9, 121.8, 93.1 59.4, 14.4; HRMS (ESI): calcd. for $\mathrm{C}_{16} \mathrm{H}_{16} \mathrm{~N}_{2} \mathrm{O}_{2}+\mathrm{H}=269.1285$, found: 269.1288 .<smiles>CCOC(=CC(Nc1ccccc1)c1ccco1)OCC</smiles>

\section{(Z)-Ethyl 3-(furan-2-yl)-3-(phenylamino)acrylate (1x)}

Yellow oil; $\mathrm{R}_{\mathrm{f}}=0.41$ (petroleum ether : EtOAc $\left.=10: 1\right) ;{ }^{1} \mathrm{H} \mathrm{NMR}\left(\mathrm{CDCl}_{3}, 400 \mathrm{MHz}, \delta\right.$ ppm): 9.93 (s, 1H), 7.34 (s, 1H), 7.25-7.18 (m, 2H), 7.04-7.01 (m, 1H), 6.82 (d, $J=$ $8.0 \mathrm{~Hz}, 2 \mathrm{H}), 6.37-6.34$ (m, 1H), 5.32 (s, 1H), 4.19 (q, J = 7.2 Hz, 2H), 1.31 (t, $J=7.2$ $\mathrm{Hz}, 3 \mathrm{H}) ;{ }^{13} \mathrm{C} \mathrm{NMR}\left(\mathrm{CDCl}_{3}, 75 \mathrm{MHz}, \delta \mathrm{ppm}\right): 170.2,148.3,147.5,143.5,140.9,128.7$, 123.7, 122.3, 112.7, 111.5, 89.0, 59.4, 14.5; HRMS (ESI): calcd. for $\mathrm{C}_{15} \mathrm{H}_{15} \mathrm{~N}_{1} \mathrm{O}_{3}+\mathrm{H}$ $=258.1125$, found: 258.1123 .<smiles>C(=C(Nc1ccccc1)c1ccccc1)c1ccccc1</smiles>

\section{(Z)-1,3-Diphenyl-3-(phenylamino)prop-2-en-1-one (3)}

${ }^{1} \mathrm{H}$ NMR $\left(\mathrm{CDCl}_{3}, 400 \mathrm{MHz}, \delta \mathrm{ppm}\right): 12.92(\mathrm{~s}, 1 \mathrm{H}), 7.97(\mathrm{~d}, J=6.8 \mathrm{~Hz}, 2 \mathrm{H})$, 7.49-7.30 (m, 8H), 7.13-7.09 (m, 2H), 7.00-6.96 (m, 1H), $6.79(\mathrm{~d}, J=8.0 \mathrm{~Hz}, 2 \mathrm{H})$, 6.09 (s, 1H); ${ }^{13} \mathrm{C} \mathrm{NMR}\left(\mathrm{CDCl}_{3}, 75 \mathrm{MHz}, \delta \mathrm{ppm}\right): 189.6,161.4,139.8,139.3,135.7$, 
$131.3,129.6,128.6,128.5,128.3,128.3,127.2,124.0,123.1,97.0$.<smiles>CCOC(=O)/C=C(/C)Nc1ccccc1</smiles>

(Z)-Ethyl 3-(phenylamino)but-2-enoate (4) ${ }^{1}$

${ }^{1} \mathrm{H} \mathrm{NMR}\left(\mathrm{CDCl}_{3}, 400 \mathrm{MHz}, \delta \mathrm{ppm}\right): 10.40$ (s, 1H), 7.33-7.29 (m, 2H), 7.16-7.12 (m, 1H), $7.08(\mathrm{~d}, J=7.6 \mathrm{~Hz}, 2 \mathrm{H}), 4.69(\mathrm{~s}, 1 \mathrm{H}), 4.15(\mathrm{q}, J=7.2 \mathrm{~Hz}, 2 \mathrm{H}), 1.99$ (s, 3H), 1.28 $(\mathrm{t}, J=7.2 \mathrm{~Hz}, 3 \mathrm{H}) ;{ }^{13} \mathrm{C} \mathrm{NMR}\left(\mathrm{CDCl}_{3}, 75 \mathrm{MHz}, \delta \mathrm{ppm}\right): 170.3,158.8,139.2,128.9$, $124.8,124.285 .9,58.6,20.2,14.5$.<smiles>CC(=Nc1ccccc1)c1ccccc1</smiles>

(E)-N-(1-phenylethylidene)aniline $(6)^{3}$

${ }^{1} \mathrm{H} \mathrm{NMR}\left(\mathrm{CDCl}_{3}, 400 \mathrm{MHz}, \delta \mathrm{ppm}\right): 7.97-7.96$ (m, 2H), 7.44-7.40 (m, 3H), 7.34 (t, $J$ $=7.6 \mathrm{~Hz}, 2 \mathrm{H}), 7.07(\mathrm{t}, J=7.6 \mathrm{~Hz}, 1 \mathrm{H}), 6.79(\mathrm{~d}, J=7.6 \mathrm{~Hz}, 2 \mathrm{H}), 2.21(\mathrm{~s}, 3 \mathrm{H}) ;{ }^{13} \mathrm{C}$ $\operatorname{NMR}\left(\mathrm{CDCl}_{3}, 75 \mathrm{MHz}, \delta \mathrm{ppm}\right): 165.4,151.6,139.3,130.4,128.9,128.3,127.1,123.1$, $119.3,17.3$.

\section{Spectroscopic data for the products}<smiles>CCOC(=O)c1nc2ccccc2nc1-c1ccccc1</smiles>

\section{Ethyl 3-phenylquinoxaline-2-carboxylate (2a)}

Yellow solid; m.p. $=65-66{ }^{\circ} \mathrm{C} ;{ }^{1} \mathrm{H}$ NMR $\left(\mathrm{CDCl}_{3}, 400 \mathrm{MHz}, \delta \mathrm{ppm}\right): 8.22(\mathrm{dd}, J=8.0$ $\mathrm{Hz}, 1.2 \mathrm{~Hz}, 1 \mathrm{H}), 8.19$ (dd, $J=8.0 \mathrm{~Hz}, 1.2 \mathrm{~Hz}, 1 \mathrm{H}), 7.88-7.79$ (m, 2H), 7.76-7.73 (m, 2H), 7.53-7.50 (m, 3H), $4.34(\mathrm{q}, J=7.2 \mathrm{~Hz}, 2 \mathrm{H}), 1.18(\mathrm{t}, J=7.2 \mathrm{~Hz}, 3 \mathrm{H}) ;{ }^{13} \mathrm{C}$ NMR $\left(\mathrm{CDCl}_{3}, 100 \mathrm{MHz}, \delta \mathrm{ppm}\right): 166.6,152.2,145.7,142.2,139.9,137.8,131.6,130.5$, 129.5, 129.3, 128.6, 128.5, 62.3, 13.7; HRMS (ESI): calcd. for $\mathrm{C}_{17} \mathrm{H}_{14} \mathrm{~N}_{2} \mathrm{O}_{2}+\mathrm{Na}=$ 301.0947, found: 301.0952 .<smiles>CCOC(=O)c1nc2cc(C)ccc2nc1-c1ccccc1</smiles>

Ethyl 7-methyl-3-phenylquinoxaline-2-carboxylate (2b)

Yellow solid; m.p. $=66-67{ }^{\circ} \mathrm{C} ;{ }^{1} \mathrm{H}$ NMR $\left(\mathrm{CDCl}_{3}, 400 \mathrm{MHz}, \delta \mathrm{ppm}\right): 8.07(\mathrm{~d}, J=8.8$ $\mathrm{Hz}, 1 \mathrm{H}), 7.98$ (s, 1H), 7.74-7.72 (m, 2H), 7.68 (dd, $J=8.4 \mathrm{~Hz}, 1.6 \mathrm{~Hz}, 1 \mathrm{H}), 7.52-7.48$ $(\mathrm{m}, 3 \mathrm{H}), 4.33(\mathrm{q}, J=7.2 \mathrm{~Hz}, 2 \mathrm{H}), 2.62(\mathrm{~s}, 3 \mathrm{H}), 1.17(\mathrm{t}, J=7.2 \mathrm{~Hz}, 3 \mathrm{H}) ;{ }^{13} \mathrm{C}$ NMR $\left(\mathrm{CDCl}_{3}, 100 \mathrm{MHz}, \delta \mathrm{ppm}\right): 166.7,151.4,145.6,141.2,140.8,140.0,137.9,134.1$, 
129.4, 128.8, 128.6, 128.5, 128.2, 62.3, 21.9, 13.7; HRMS (ESI): calcd. for $\mathrm{C}_{18} \mathrm{H}_{16} \mathrm{~N}_{2} \mathrm{O}_{2}+\mathrm{Na}=315.1104$, found: 315.1111 .<smiles>CCOC(=O)c1nc2cc(OC)ccc2nc1-c1ccccc1</smiles>

\section{Ethyl 7-methoxy-3-phenylquinoxaline-2-carboxylate (2c)}

Yellow solid; m.p. $=114-116{ }^{\circ} \mathrm{C} ;{ }^{1} \mathrm{H} \mathrm{NMR}\left(\mathrm{CDCl}_{3}, 400 \mathrm{MHz}, \delta \mathrm{ppm}\right): 8.05(\mathrm{dd}, J=$ $8.0 \mathrm{~Hz}, 2.0 \mathrm{~Hz}, 2 \mathrm{H}), 7.73-7.71(\mathrm{~m}, 2 \mathrm{H}), 7.51-7.48(\mathrm{~m}, 5 \mathrm{H}), 4.33$ (q, J = 7.2 Hz, 2H), $3.97(\mathrm{~s}, 3 \mathrm{H}), 1.18(\mathrm{t}, J=7.2 \mathrm{~Hz}, 3 \mathrm{H}) ;{ }^{13} \mathrm{C} \mathrm{NMR}\left(\mathrm{CDCl}_{3}, 100 \mathrm{MHz}, \delta \mathrm{ppm}\right): 166.7$, $161.1,149.7,145.3,141.5,138.5,137.9,130.1,129.1,128.5,128.4,125.3,106.4$, 62.2, 55.8, 13.7; HRMS (ESI): calcd. for $\mathrm{C}_{18} \mathrm{H}_{16} \mathrm{~N}_{2} \mathrm{O}_{3}+\mathrm{H}=309.1234$, found: 309.1238 .<smiles>CCOC(=O)c1nc2cc(F)ccc2nc1-c1ccccc1</smiles>

\section{Ethyl 7-fluoro-3-phenylquinoxaline-2-carboxylate (2d)}

Yellow solid; m.p. $=76-77{ }^{\circ} \mathrm{C} ;{ }^{1} \mathrm{H}$ NMR $\left(\mathrm{CDCl}_{3}, 400 \mathrm{MHz}, \delta\right.$ ppm): 8.19 (dd, $J=9.2$ $\mathrm{Hz}, 5.6 \mathrm{~Hz}, 1 \mathrm{H}), 7.84(\mathrm{dd}, J=8.8 \mathrm{~Hz}, 2.8 \mathrm{~Hz}, 1 \mathrm{H}), 7.75-7.72(\mathrm{~m}, 2 \mathrm{H}), 7.67-7.62$ (m, 1H), 7.53-7.51 (m, 3H), $4.34(\mathrm{q}, J=7.2 \mathrm{~Hz}, 2 \mathrm{H}), 1.18(\mathrm{t}, J=7.2 \mathrm{~Hz}, 3 \mathrm{H}) ;{ }^{13} \mathrm{C} \mathrm{NMR}$ $\left(\mathrm{CDCl}_{3}, 100 \mathrm{MHz}, \delta \mathrm{ppm}\right): 166.4,163.0(\mathrm{~d}, J=252 \mathrm{~Hz}), 151.6,151.5,146.5,140.7$, $140.6,139.5$, 137.4, 131.5, 131.4, 129.7, 128.7, 128.5, 122.4, 122.1, 113.0, 112.8, 62.5, 13.7; HRMS (ESI): calcd. for $\mathrm{C}_{17} \mathrm{H}_{13} \mathrm{~F}_{1} \mathrm{~N}_{2} \mathrm{O}_{2}+\mathrm{H}=297.1034$, found: 297.1038 .<smiles>CCOC(=O)c1nc2cc(Cl)ccc2nc1-c1ccccc1</smiles>

\section{Ethyl 7-chloro-3-phenylquinoxaline-2-carboxylate (2e)}

Yellow solid; m.p. $=91-92{ }^{\circ} \mathrm{C} ;{ }^{1} \mathrm{H}$ NMR $\left(\mathrm{CDCl}_{3}, 400 \mathrm{MHz}, \delta \mathrm{ppm}\right): 8.20(\mathrm{~d}, J=2.0$ $\mathrm{Hz}, 1 \mathrm{H}), 8.11(\mathrm{~d}, J=9.2 \mathrm{~Hz}, 1 \mathrm{H}), 7.79(\mathrm{dd}, J=9.2 \mathrm{~Hz}, 2.4 \mathrm{~Hz}, 1 \mathrm{H}), 7.75-7.73$ (m, $2 \mathrm{H}), 7.52(\mathrm{t}, J=3.2 \mathrm{~Hz}, 3 \mathrm{H}), 4.34(\mathrm{q}, J=7.2 \mathrm{~Hz}, 2 \mathrm{H}), 1.19(\mathrm{t}, J=7.2 \mathrm{~Hz}, 3 \mathrm{H}) ;{ }^{13} \mathrm{C}$ NMR $\left(\mathrm{CDCl}_{3}, 100 \mathrm{MHz}, \delta \mathrm{ppm}\right): 166.3,152.3,146.6,140.7,140.1,137.3,136.4$, 132.7, 130.5, 129.8, 128.7, 128.5, 128.3, 62.5, 13.7; HRMS (ESI): calcd. for $\mathrm{C}_{17} \mathrm{H}_{13} \mathrm{Cl}_{1} \mathrm{~N}_{2} \mathrm{O}_{2}+\mathrm{H}=313.0738$, found: 313.0742 .<smiles>CCOC(=O)c1nc2cc(Br)ccc2nc1-c1ccccc1</smiles>

Ethyl 7-bromo-3-phenylquinoxaline-2-carboxylate (2f)

Yellow solid; m.p. $=103-104{ }^{\circ} \mathrm{C} ;{ }^{1} \mathrm{H}$ NMR $\left(\mathrm{CDCl}_{3}, 400 \mathrm{MHz}, \delta \mathrm{ppm}\right): 8.39(\mathrm{~d}, J=$ 
$2.0 \mathrm{~Hz}, 1 \mathrm{H}), 8.05(\mathrm{~d}, J=8.8 \mathrm{~Hz}, 1 \mathrm{H}), 7.92(\mathrm{dd}, J=8.8 \mathrm{~Hz}, 2.0 \mathrm{~Hz}, 1 \mathrm{H}), 7.75-7.73(\mathrm{~m}$, 2H), 7.53-7.51 (m, 3H), $4.34(\mathrm{q}, J=7.2 \mathrm{~Hz}, 2 \mathrm{H}), 1.19(\mathrm{t}, J=7.2 \mathrm{~Hz}, 3 \mathrm{H}) ;{ }^{13} \mathrm{C}$ NMR $\left(\mathrm{CDCl}_{3}, 100 \mathrm{MHz}, \delta \mathrm{ppm}\right): 166.3,152.4,146.5,141.0,140.4,137.4,135.2,131.7$, 130.6, 129.8, 128.7, 128.5, 124.5, 62.5, 13.7; HRMS (ESI): calcd. for $\mathrm{C}_{17} \mathrm{H}_{13} \mathrm{Br}_{1} \mathrm{~N}_{2} \mathrm{O}_{2}$

$+\mathrm{Na}=379.0053$, found: 379.0058 .<smiles>CCOC(=O)c1nc2cc(I)ccc2nc1-c1ccccc1</smiles>

\section{Ethyl 7-iodo-3-phenylquinoxaline-2-carboxylate (2g)}

Yellow solid; m.p. $=104-106{ }^{\circ} \mathrm{C} ;{ }^{1} \mathrm{H}$ NMR $\left(\mathrm{CDCl}_{3}, 400 \mathrm{MHz}, \delta \mathrm{ppm}\right): 8.63(\mathrm{~d}, J=$ $2.0 \mathrm{~Hz}, 1 \mathrm{H}), 8.08$ (dd, $J=8.8 \mathrm{~Hz}, 1.6 \mathrm{~Hz}, 1 \mathrm{H}), 7.88(\mathrm{~d}, J=8.8 \mathrm{~Hz}, 1 \mathrm{H}), 7.75-7.73(\mathrm{~m}$, 2H), 7.53-7.50 (m, 3H), $4.34(\mathrm{q}, J=7.2 \mathrm{~Hz}, 2 \mathrm{H}), 1.19(\mathrm{t}, J=7.2 \mathrm{~Hz}, 3 \mathrm{H}) ;{ }^{13} \mathrm{C}$ NMR $\left(\mathrm{CDCl}_{3}, 100 \mathrm{MHz}, \delta \mathrm{ppm}\right): 166.2,152.5,146.3,141.4,140.5,140.4,138.4,137.4$, 130.5, 129.8, 128.7, 128.5, 96.3, 62.5, 13.7; HRMS (ESI): calcd. for $\mathrm{C}_{17} \mathrm{H}_{13} \mathrm{I}_{1} \mathrm{~N}_{2} \mathrm{O}_{2}+$ $\mathrm{H}=405.0094$, found: 405.0101 .<smiles>CCOC(=O)c1nc2cc(C(F)(F)F)ccc2nc1-c1ccccc1</smiles>

Ethyl 3-phenyl-7-(trifluoromethyl)quinoxaline-2-carboxylate (2h)

Yellow solid; m.p. $=114-117{ }^{\circ} \mathrm{C} ;{ }^{1} \mathrm{H}$ NMR $\left(\mathrm{CDCl}_{3}, 400 \mathrm{MHz}, \delta \mathrm{ppm}\right): 8.54(\mathrm{~s}, 1 \mathrm{H})$, $8.31(\mathrm{~d}, J=8.8 \mathrm{~Hz}, 1 \mathrm{H}), 8.02(\mathrm{dd}, J=8.8 \mathrm{~Hz}, 2.0 \mathrm{~Hz}, 1 \mathrm{H}), 7.79-7.77(\mathrm{~m}, 2 \mathrm{H})$, $7.56-7.53(\mathrm{~m}, 3 \mathrm{H}), 4.37$ (q, $J=7.2 \mathrm{~Hz}, 2 \mathrm{H}), 1.21(\mathrm{t}, J=7.2 \mathrm{~Hz}, 3 \mathrm{H}) ;{ }^{13} \mathrm{C} \mathrm{NMR}$ $\left(\mathrm{CDCl}_{3}, 100 \mathrm{MHz}, \delta \mathrm{ppm}\right): 166.0,154.0,147.1,143.2,138.8,137.0,132.4$ (q, $J=33$ $\mathrm{Hz}), 130.6$, 130.1, 128.7, 128.6, 127.6, 127.5, 127.2, 127.2, 124.8, 122.1, 62.6, 13.7; HRMS (ESI): calcd. for $\mathrm{C}_{18} \mathrm{H}_{13} \mathrm{~F}_{3} \mathrm{~N}_{2} \mathrm{O}_{2}+\mathrm{H}=347.1002$, found: 347.1009 .<smiles>CCOC(=O)c1nc2cc(-c3ccccc3)ccc2nc1-c1ccccc1</smiles>

\section{Ethyl 3,7-diphenylquinoxaline-2-carboxylate (2i)}

Pale yellow solid; m.p. $=136-139{ }^{\circ} \mathrm{C} ;{ }^{1} \mathrm{H}$ NMR $\left(\mathrm{CDCl}_{3}, 400 \mathrm{MHz}, \delta \mathrm{ppm}\right): 8.43(\mathrm{~d}, J$ $=2.0 \mathrm{~Hz}, 1 \mathrm{H}), 8.24(\mathrm{~d}, J=8.8 \mathrm{~Hz}, 1 \mathrm{H}), 8.14(\mathrm{dd}, J=8.8 \mathrm{~Hz}, 2.8 \mathrm{~Hz}, 1 \mathrm{H}), 7.78-7.76$ (m, 4H), 7.55-7.51 (m, 5H), $7.44(\mathrm{t}, J=7.2 \mathrm{~Hz}, 1 \mathrm{H}), 4.35(\mathrm{q}, J=7.2 \mathrm{~Hz}, 2 \mathrm{H}), 1.19(\mathrm{t}$, $J=7.2 \mathrm{~Hz}, 3 \mathrm{H}) ;{ }^{13} \mathrm{C} \mathrm{NMR}\left(\mathrm{CDCl}_{3}, 100 \mathrm{MHz}, \delta \mathrm{ppm}\right): 166.6,152.0,146.1,143.2$, 141.6, 140.1, 139.2, 137.8, 131.4, 129.6, 129.5, 129.2, 128.6, 128.5, 128.4, 127.4, 126.7, 62.4, 13.7; HRMS (ESI): calcd. for $\mathrm{C}_{23} \mathrm{H}_{18} \mathrm{~N}_{2} \mathrm{O}_{2}+\mathrm{H}=355.1441$, found: 355.1439 . 
<smiles>CCOC(=O)c1nc2c([N+](=O)[O-])cccc2nc1-c1ccccc1</smiles>

Ethyl 8-methyl-3-phenylquinoxaline-2-carboxylate (2j-1)

White solid; m.p. $=83-85{ }^{\circ} \mathrm{C} ;{ }^{1} \mathrm{H}$ NMR $\left(\mathrm{CDCl}_{3}, 400 \mathrm{MHz}, \delta \mathrm{ppm}\right): 8.01$ (d, $J=8.4$ $\mathrm{Hz}, 1 \mathrm{H}), 7.77-7.74(\mathrm{~m}, 2 \mathrm{H}), 7.72(\mathrm{~d}, J=8.4 \mathrm{~Hz}, 1 \mathrm{H}), 7.63(\mathrm{~d}, J=6.8 \mathrm{~Hz}, 1 \mathrm{H})$, $7.52-7.49(\mathrm{~m}, 3 \mathrm{H}), 4.31(\mathrm{q}, J=7.2 \mathrm{~Hz}, 2 \mathrm{H}), 2.85(\mathrm{~s}, 3 \mathrm{H}), 1.14(\mathrm{t}, J=7.2 \mathrm{~Hz}, 3 \mathrm{H}) ;{ }^{13} \mathrm{C}$ NMR $\left(\mathrm{CDCl}_{3}, 100 \mathrm{MHz}, \delta \mathrm{ppm}\right): 167.0,151.5,144.6,142.4,139.2,138.2,137.9$, 131.3, 130.3, 129.4, 128.6, 128.5, 127.1, 62.1, 17.1, 13.7; HRMS (ESI): calcd. for $\mathrm{C}_{18} \mathrm{H}_{16} \mathrm{~N}_{2} \mathrm{O}_{2}+\mathrm{Na}=315.1104$, found: 315.1110 .<smiles>CCOC(=O)c1nc2ccc(C)cc2nc1-c1ccccc1</smiles>

\section{Ethyl 6-methyl-3-phenylquinoxaline-2-carboxylate $(2 \mathbf{j}-2)$}

White solid; m.p. $=83-85{ }^{\circ} \mathrm{C} ;{ }^{1} \mathrm{H}$ NMR $\left(\mathrm{CDCl}_{3}, 400 \mathrm{MHz}, \delta \mathrm{ppm}\right): 8.10(\mathrm{~d}, J=8.4$ $\mathrm{Hz}, 1 \mathrm{H}), 7.95(\mathrm{~s}, 1 \mathrm{H}), 7.74-7.71(\mathrm{~m}, 2 \mathrm{H}), 7.65(\mathrm{dd}, J=8.4 \mathrm{~Hz}, 1.2 \mathrm{~Hz}, 1 \mathrm{H})$, $7.52-7.49(\mathrm{~m}, 3 \mathrm{H}), 4.32(\mathrm{q}, J=7.2 \mathrm{~Hz}, 2 \mathrm{H}), 2.63(\mathrm{~s}, 3 \mathrm{H}), 1.17(\mathrm{t}, J=7.2 \mathrm{~Hz}, 3 \mathrm{H}) ;{ }^{13} \mathrm{C}$ $\mathrm{NMR}\left(\mathrm{CDCl}_{3}, 100 \mathrm{MHz}, \delta \mathrm{ppm}\right): 166.7,152.3,144.7,142.6,142.4,138.4,138.0$, 132.9, 129.4, 129.1, 128.5, 128.1, 62.3, 22.0, 13.7; HRMS (ESI): calcd. for $\mathrm{C}_{18} \mathrm{H}_{16} \mathrm{~N}_{2} \mathrm{O}_{2}+\mathrm{Na}=315.1104$, found: 315.1109 .<smiles>CCOC(=O)c1nc2c(Cl)cccc2nc1-c1ccccc1</smiles>

\section{Ethyl 8-chloro-3-phenylquinoxaline-2-carboxylate (2k-1)}

\section{Ethyl 6-chloro-3-phenylquinoxaline-2-carboxylate (2k-2)}

Yellow solid; m.p. $=67-69{ }^{\circ} \mathrm{C} ;{ }^{1} \mathrm{H}$ NMR $\left(\mathrm{CDCl}_{3}, 400 \mathrm{MHz}, \delta \mathrm{ppm}\right): 8.17(\mathrm{~d}, J=2.4$ $\mathrm{Hz}, 0.23 \mathrm{H}), 8.15(\mathrm{~d}, J=9.2 \mathrm{~Hz}, 0.28 \mathrm{H}), 8.10(\mathrm{dd}, J=8.4 \mathrm{~Hz}, 1.2 \mathrm{~Hz}, 0.74 \mathrm{H}), 7.90$ (dd, $J=7.6 \mathrm{~Hz}, 1.2 \mathrm{~Hz}, 0.71 \mathrm{H}), 7.78-7.73(\mathrm{~m}, 3 \mathrm{H}), 7.53-7.51(\mathrm{~m}, 3 \mathrm{H}), 4.37-4.31(\mathrm{~m}, 2 \mathrm{H})$, $1.20-1.15(\mathrm{~m}, 3 \mathrm{H}) ;{ }^{13} \mathrm{C} \mathrm{NMR}\left(\mathrm{CDCl}_{3}, 100 \mathrm{MHz}, \delta \mathrm{ppm}\right): 166.3,166.1,153.0,152.7$, $146.1,145.8,143.1,142.5,138.3,137.7,137.3,137.1,136.9,133.5,131.6,131.2$, 130.7, 130.3, 129.9, 129.8, 128.7, 128.6, 128.6, 128.5, 128.3, 128.2, 62.5, 62.4, 13.7; HRMS (ESI): calcd. for $\mathrm{C}_{17} \mathrm{H}_{13} \mathrm{Cl}_{1} \mathrm{~N}_{2} \mathrm{O}_{2}+\mathrm{Na}=335.0558$, found: 335.0563 .<smiles>CCOC(=O)c1nc2cccc([N+](=O)[O-])c2nc1-c1ccccc1</smiles>

Ethyl 5-methyl-3-phenylquinoxaline-2-carboxylate (2I) 
Yellow oil; $\mathrm{R}_{\mathrm{f}}=0.50$ (petroleum ether : EtOAc $\left.=10: 1\right) ;{ }^{1} \mathrm{H} \mathrm{NMR}\left(\mathrm{CDCl}_{3}, 400 \mathrm{MHz}, \delta\right.$ ppm): 8.05-8.03 (m, 1H), 7.81-7.79 (m, 2H), 7.71-7.67 (m, 2H), 7.51-7.50 (m, 3H), $4.34(\mathrm{q}, J=7.2 \mathrm{~Hz}, 2 \mathrm{H}), 2.84(\mathrm{~s}, 3 \mathrm{H}), 1.19(\mathrm{t}, J=7.2 \mathrm{~Hz}, 3 \mathrm{H}) ;{ }^{13} \mathrm{C} \mathrm{NMR}_{\left(\mathrm{CDCl}_{3}, 100\right.}$ $\mathrm{MHz}, \delta$ ppm): 167.0, 150.6, 145.1, 141.4, 139.9, 138.1, 137.8, 131.4, 130.2, 129.4, 128.8, 128.5, 127.3, 62.3, 17.0, 13.7; HRMS (ESI): calcd. for $\mathrm{C}_{18} \mathrm{H}_{16} \mathrm{~N}_{2} \mathrm{O}_{2}+\mathrm{Na}=$ 315.1104, found: 315.1108 .<smiles>CCOC(=O)c1nc2cccc(Cl)c2nc1-c1ccccc1</smiles>

\section{Ethyl 5-chloro-3-phenylquinoxaline-2-carboxylate (2n)}

Yellow solid; m.p. $=117-120{ }^{\circ} \mathrm{C} ;{ }^{1} \mathrm{H} \mathrm{NMR}\left(\mathrm{CDCl}_{3}, 400 \mathrm{MHz}, \delta \mathrm{ppm}\right): 8.14(\mathrm{dd}, J=$ $8.4 \mathrm{~Hz}, 1.2 \mathrm{~Hz}, 1 \mathrm{H}), 7.96-7.94(\mathrm{~m}, 1 \mathrm{H}), 7.85-7.82(\mathrm{~m}, 2 \mathrm{H}), 7.73(\mathrm{t}, J=8.0 \mathrm{~Hz}, 1 \mathrm{H})$, $7.53-7.52(\mathrm{~m}, 3 \mathrm{H}), 4.36(\mathrm{q}, J=7.2 \mathrm{~Hz}, 2 \mathrm{H}), 1.20(\mathrm{t}, J=7.2 \mathrm{~Hz}, 3 \mathrm{H}) ;{ }^{13} \mathrm{C} \mathrm{NMR}$ $\left(\mathrm{CDCl}_{3}, 100 \mathrm{MHz}, \delta \mathrm{ppm}\right): 166.4,152.2,146.3,140.7,139.0,137.2,133.3,131.4$, 130.0, 129.9, 128.8, 128.7, 128.5, 62.5, 13.7; HRMS (ESI): calcd. for $\mathrm{C}_{17} \mathrm{H}_{13} \mathrm{Cl}_{1} \mathrm{~N}_{2} \mathrm{O}_{2}$ $+\mathrm{H}=313.0738$, found: 313.0736 .<smiles>CCOC(=O)c1nc2cccc(Br)c2nc1-c1ccccc1</smiles>

\section{Ethyl 5-bromo-3-phenylquinoxaline-2-carboxylate (2o)}

Red solid; m.p. $=104-107{ }^{\circ} \mathrm{C} ;{ }^{1} \mathrm{H}$ NMR $\left(\mathrm{CDCl}_{3}, 400 \mathrm{MHz}, \delta \mathrm{ppm}\right)$ : 8.19-8.15 (m, 2H), 7.87-7.84 (m, 2H), 7.69-7.65 (m, 1H), 7.54-7.52 (m, 3H), 4.36 (q, J = 7.2 Hz, 2H), $1.20(\mathrm{t}, J=7.2 \mathrm{~Hz}, 3 \mathrm{H}) ;{ }^{13} \mathrm{C} \mathrm{NMR}\left(\mathrm{CDCl}_{3}, 100 \mathrm{MHz}, \delta \mathrm{ppm}\right): 166.4,152.4$, 146.3 , 140.7, 139.9, 137.1, 134.9, 130.6, 130.0, 129.2, 128.9, 128.7, 124.3, 62.6, 13.7; HRMS (ESI): calcd. for $\mathrm{C}_{17} \mathrm{H}_{13} \mathrm{Br}_{1} \mathrm{~N}_{2} \mathrm{O}_{2}+\mathrm{H}=357.0233$, found: 357.0229 .<smiles>CCOC(=O)c1nc2cccc(C(F)(F)F)c2nc1-c1ccccc1</smiles>

\section{Ethyl 3-phenyl-5-(trifluoromethyl)quinoxaline-2-carboxylate (2p)}

Yellow solid; m.p. $=75-77{ }^{\circ} \mathrm{C} ;{ }^{1} \mathrm{H}$ NMR $\left(\mathrm{CDCl}_{3}, 400 \mathrm{MHz}, \delta\right.$ ppm): 8.38 (d, $J=8.4$ $\mathrm{Hz}, 1 \mathrm{H}), 8.19(\mathrm{~d}, J=7.2 \mathrm{~Hz}, 1 \mathrm{H}), 7.87-7.83(\mathrm{~m}, 3 \mathrm{H}), 7.54-7.52(\mathrm{~m}, 3 \mathrm{H}), 4.38(\mathrm{q}, J=$ $7.2 \mathrm{~Hz}, 2 \mathrm{H}), 1.21(\mathrm{t}, J=7.2 \mathrm{~Hz}, 3 \mathrm{H}) ;{ }^{13} \mathrm{C} \mathrm{NMR}\left(100 \mathrm{MHz}, \mathrm{CDCl}_{3}, \delta \mathrm{ppm}\right): 166.4$, 152.0, 146.3, 139.6, 139.1, 136.9, 133.7, 130.2, 129.7, 129.7, 128.9, 128.7, 128.1 (q, $J$ $=30 \mathrm{~Hz}$ ), 124.7, 122.0, 62.6, 13.7; HRMS (ESI): calcd. for $\mathrm{C}_{18} \mathrm{H}_{13} \mathrm{~F}_{3} \mathrm{~N}_{2} \mathrm{O}_{2}+\mathrm{H}=$ 347.1002, found: 347.1006 . 
<smiles>CCOC(=O)c1nc2c([N+](=O)[O-])cc(C)cc2nc1-c1ccccc1</smiles>

Ethyl 6,8-dimethyl-3-phenylquinoxaline-2-carboxylate (2q)

Yellow solid; m.p. $=111-113{ }^{\circ} \mathrm{C} ;{ }^{1} \mathrm{H}$ NMR $\left(\mathrm{CDCl}_{3}, 400 \mathrm{MHz}, \delta \mathrm{ppm}\right): 7.78(\mathrm{~s}, 1 \mathrm{H})$, 7.74-7.72 (m, 2H), 7.50-7.47 (m, 4H), 4.30 (q, J=7.2 Hz, 2H), $2.81(\mathrm{~s}, 3 \mathrm{H}), 2.56(\mathrm{~S}$, $3 \mathrm{H}), 1.13(\mathrm{t}, J=7.2 \mathrm{~Hz}, 3 \mathrm{H}) ;{ }^{13} \mathrm{C} \mathrm{NMR}\left(100 \mathrm{MHz}, \mathrm{CDCl}_{3}, \delta \mathrm{ppm}\right): 167.1,151.6$, 143.6, 142.6, 142.1, 138.1, 137.8, 137.5, 132.8, 129.3, 128.5, 128.5, 125.9, 62.0, 22.0, 17.0, 13.7; HRMS (ESI): calcd. for $\mathrm{C}_{19} \mathrm{H}_{18} \mathrm{~N}_{2} \mathrm{O}_{2}+\mathrm{H}=307.1441$, found: 307.1447 .<smiles>CCOC(=O)c1nc2c(C(F)(F)F)cc(C(F)(F)F)cc2nc1-c1ccccc1</smiles>

\section{Ethyl 3-phenyl-6,8-bis(trifluoromethyl)quinoxaline-2-carboxylate (2r)}

Pale yellow solid; m.p. $=77-78{ }^{\circ} \mathrm{C} ;{ }^{1} \mathrm{H} \mathrm{NMR}\left(\mathrm{CDCl}_{3}, 400 \mathrm{MHz}, \delta \mathrm{ppm}\right): 8.69(\mathrm{~s}, 1 \mathrm{H})$, $8.31(\mathrm{~s}, 1 \mathrm{H}), 7.82-7.79(\mathrm{~m}, 2 \mathrm{H}), 7.57-7.55(\mathrm{~m}, 3 \mathrm{H}), 4.39$ (q, J = 7.2 Hz, 2H), 1.22 (t, $J=7.2 \mathrm{~Hz}, 3 \mathrm{H}) ;{ }^{13} \mathrm{C} \mathrm{NMR}\left(100 \mathrm{MHz}, \mathrm{CDCl}_{3}, \delta \mathrm{ppm}\right): 165.6,153.9,148.2,141.5$, 137.9, 136.2, 132.0 (q, $J=34 \mathrm{~Hz}), 131.5,131.5,130.6,130.0$ (q, $J=31 \mathrm{~Hz}), 128.9$, 128.7, 124.4, 124.2, 123.8, 121.5, 121.1, 62.8, 13.7; HRMS (ESI): calcd. for $\mathrm{C}_{19} \mathrm{H}_{12} \mathrm{~F}_{6} \mathrm{~N}_{2} \mathrm{O}_{2}+\mathrm{H}=415.0876$, found: 415.0883 .<smiles>CCOC(=O)c1nc2c(ccc3ccccc32)nc1-c1ccccc1</smiles>

\section{Ethyl 3-phenylbenzo[f]quinoxaline-2-carboxylate (2s)}

White solid; m.p. $=120-122{ }^{\circ} \mathrm{C} ;{ }^{1} \mathrm{H}$ NMR $\left(\mathrm{CDCl}_{3}, 400 \mathrm{MHz}, \delta \mathrm{ppm}\right): 9.28-9.26(\mathrm{~m}$, $1 \mathrm{H}), 8.10(\mathrm{~d}, J=9.2 \mathrm{~Hz}, 1 \mathrm{H}), 8.00(\mathrm{~d}, J=9.2 \mathrm{~Hz}, 1 \mathrm{H}), 7.94(\mathrm{dd}, J=6.8 \mathrm{~Hz}, 1.6 \mathrm{~Hz}$, 1H), 7.81-7.75 (m, 4H), 7.53-7.51 (m, 3H), $4.36(\mathrm{q}, J=7.2 \mathrm{~Hz}, 2 \mathrm{H}), 1.18(\mathrm{t}, J=7.2$ $\mathrm{Hz}, 3 \mathrm{H}) ;{ }^{13} \mathrm{C}$ NMR $\left(100 \mathrm{MHz}, \mathrm{CDCl}_{3}, \delta \mathrm{ppm}\right): 167.1,151.9,143.6,142.3,138.7$, $137.9,133.5,133.3,130.4,129.4,129.3,128.7,128.6,128.0,127.9,126.4,125.0$, 62.1, 13.7; HRMS (ESI): calcd. for $\mathrm{C}_{21} \mathrm{H}_{16} \mathrm{~N}_{2} \mathrm{O}_{2}+\mathrm{H}=329.1285$, found: 329.1281 .<smiles></smiles>

\section{Ethyl 2-phenylbenzo[f]quinoxaline-3-carboxylate (2t)}

Yellow solid; m.p. $=85-87{ }^{\circ} \mathrm{C} ;{ }^{1} \mathrm{H} \mathrm{NMR}\left(\mathrm{CDCl}_{3}, 400 \mathrm{MHz}, \delta \mathrm{ppm}\right)$ : 9.33-9.31 (m, 1H), 8.10-8.04 (m, 2H), 7.99-7.96 (m, 1H), 7.89-7.86 (m, 2H), 7.80-7.78 (m, 2H), $7.56-7.54(\mathrm{~m}, 3 \mathrm{H}), 4.37(\mathrm{q}, J=7.2 \mathrm{~Hz}, 2 \mathrm{H}), 1.21(\mathrm{t}, J=7.2 \mathrm{~Hz}, 3 \mathrm{H}) ;{ }^{13} \mathrm{C}$ NMR $(100$ 
$\left.\mathrm{MHz}, \mathrm{CDCl}_{3}, \delta \mathrm{ppm}\right): 167.0,150.7,144.5,141.2,139.6,138.0,134.0,132.3,130.5$, 129.7, 129.5, 128.9, 128.5, 128.1, 127.7, 126.3, 125.2, 62.3, 13.8; HRMS (ESI): calcd. for $\mathrm{C}_{21} \mathrm{H}_{16} \mathrm{~N}_{2} \mathrm{O}_{2}+\mathrm{H}=329.1285$, found: 329.1290 .<smiles>CCOC(=O)c1nc2ccccc2nc1-c1ccc(OC)cc1</smiles>

Ethyl 3-(4-methoxyphenyl)quinoxaline-2-carboxylate (2u)

Yellow solid; m.p. $=120-122{ }^{\circ} \mathrm{C} ;{ }^{1} \mathrm{H} \mathrm{NMR}\left(\mathrm{CDCl}_{3}, 400 \mathrm{MHz}, \delta \mathrm{ppm}\right): 8.19(\mathrm{dd}, J=$ $8.4 \mathrm{~Hz}, 1.2 \mathrm{~Hz}, 1 \mathrm{H}), 8.15$ (dd, $J=8.4 \mathrm{~Hz}, 1.2 \mathrm{~Hz}, 1 \mathrm{H}), 7.86-7.81(\mathrm{~m}, 1 \mathrm{H}), 7.80-7.76$ z (m, 1H), 7.75-7.72 (m, 2H), 7.05-7.03 (m, 2H), $4.38(\mathrm{q}, J=7.2 \mathrm{~Hz}, 2 \mathrm{H}), 3.88(\mathrm{~s}$, $3 \mathrm{H}), 1.25(\mathrm{t}, J=7.2 \mathrm{~Hz}, 3 \mathrm{H}) ;{ }^{13} \mathrm{C} \mathrm{NMR}\left(100 \mathrm{MHz}, \mathrm{CDCl}_{3}, \delta \mathrm{ppm}\right): 166.9,160.9$, 151.6, 145.7, 142.3, 139.6, 131.5, 130.1, 130.1, 130.0, 129.4, 129.2, 114.1, 62.4, 55.4, 13.8; HRMS (ESI): calcd. for $\mathrm{C}_{18} \mathrm{H}_{16} \mathrm{~N}_{2} \mathrm{O}_{3}+\mathrm{H}=309.1234$, found: 309.1238 .<smiles>CCOC(=O)c1nc2ccccc2nc1-c1ccc(Br)cc1</smiles>

\section{Ethyl 3-(4-bromophenyl)quinoxaline-2-carboxylate (2v)}

White solid; m.p. $=137-138{ }^{\circ} \mathrm{C} ;{ }^{1} \mathrm{H}$ NMR $\left(\mathrm{CDCl}_{3}, 400 \mathrm{MHz}, \delta \mathrm{ppm}\right): 8.24-8.21(\mathrm{~m}$, $1 \mathrm{H}), 8.18-8.16(\mathrm{~m}, 1 \mathrm{H}), 7.89-7.82(\mathrm{~m}, 2 \mathrm{H}), 7.67-7.62(\mathrm{~m}, 4 \mathrm{H}), 4.37$ (q, $J=7.2 \mathrm{~Hz}$, $2 \mathrm{H}), 1.25(\mathrm{t}, J=7.2 \mathrm{~Hz}, 3 \mathrm{H}) ;{ }^{13} \mathrm{C} \mathrm{NMR}\left(100 \mathrm{MHz}, \mathrm{CDCl}_{3}, \delta \mathrm{ppm}\right): 166.3,151.1$, 145.2, 142.2, 139.9, 136.7, 131.9, 131.8, 130.8, 130.2, 129.6, 129.3, 124.3, 62.6, 13.8; HRMS (ESI): calcd. for $\mathrm{C}_{18} \mathrm{H}_{16} \mathrm{~N}_{2} \mathrm{O}_{3}+\mathrm{H}=309.1234$, found: 309.1238 .<smiles>CCOC(=O)c1nc2ccccc2nc1-c1ccccn1</smiles>

\section{Ethyl 3-(pyridin-2-yl)quinoxaline-2-carboxylate (2w)}

White solid; m.p. $=82-83{ }^{\circ} \mathrm{C} ;{ }^{1} \mathrm{H}$ NMR $\left(\mathrm{CDCl}_{3}, 400 \mathrm{MHz}, \delta \mathrm{ppm}\right)$ : 8.67-8.66 (m, $1 \mathrm{H}), 8.43(\mathrm{~d}, J=8.0 \mathrm{~Hz}, 1 \mathrm{H}), 8.22-8.16(\mathrm{~m}, 2 \mathrm{H}), 7.91(\mathrm{td}, J=8.0 \mathrm{~Hz}, 1.6 \mathrm{~Hz}, 1 \mathrm{H})$, 7.87-7.80 (m, 2H), 4.49 (q, $J=7.2 \mathrm{~Hz}, 2 \mathrm{H}), 1.35(\mathrm{t}, J=7.2 \mathrm{~Hz}, 3 \mathrm{H}) ;{ }^{13} \mathrm{C}$ NMR $(100$ $\left.\mathrm{MHz}, \mathrm{CDCl}_{3}, \delta \mathrm{ppm}\right): 167.1,154.2,149.0,148.5,146.5,141.5,140.4,137.0,131.3$, 130.8, 129.4, 129.3, 124.4, 122.8, 62.1, 13.9; HRMS (ESI): calcd. for $\mathrm{C}_{16} \mathrm{H}_{13} \mathrm{~N}_{3} \mathrm{O}_{2}+$ $\mathrm{H}=280.1081$, found: 280.1079 .<smiles>CCOC(=O)c1nc2ccccc2nc1-c1ccco1</smiles>

\section{Ethyl 3-(furan-2-yl)quinoxaline-2-carboxylate (2x)}

Brown solid; m.p. $=83-85{ }^{\circ} \mathrm{C} ;{ }^{1} \mathrm{H}$ NMR $\left(\mathrm{CDCl}_{3}, 400 \mathrm{MHz}, \delta \mathrm{ppm}\right): 8.12(\mathrm{~d}, J=8.4$ 
$\mathrm{Hz}, 2 \mathrm{H}), 7.85-7.81(\mathrm{~m}, 1 \mathrm{H}), 7.79-7.75(\mathrm{~m}, 1 \mathrm{H}), 7.64(\mathrm{~s}, 1 \mathrm{H}), 7.29(\mathrm{~d}, J=3.6 \mathrm{~Hz}, 1 \mathrm{H})$, 6.63-6.62 (m, 1H), $4.56(\mathrm{q}, J=7.2 \mathrm{~Hz}, 2 \mathrm{H}), 1.43(\mathrm{t}, J=7.2 \mathrm{~Hz}, 3 \mathrm{H}) ;{ }^{13} \mathrm{C}$ NMR $(100$ $\left.\mathrm{MHz}, \mathrm{CDCl}_{3}, \delta \mathrm{ppm}\right): 166.5,150.6,145.0,144.1,141.9,140.8,139.5,131.6,130.3$, 129.4, 129.0, 113.1, 112.5, 62.6, 14.1; HRMS (ESI): calcd. for $\mathrm{C}_{15} \mathrm{H}_{12} \mathrm{~N}_{2} \mathrm{O}_{3}+\mathrm{H}=$ 269.0921, found: 269.0923 .<smiles>O=C(Oc1ccccc1)c1nc2ccccc2nc1-c1ccccc1</smiles>

\section{Phenyl(3-phenylquinoxalin-2-yl)methanone (7)}

Yellow solid; m.p. $=139-141{ }^{\circ} \mathrm{C} ;{ }^{1} \mathrm{H}$ NMR $\left(\mathrm{CDCl}_{3}, 400 \mathrm{MHz}, \delta\right.$ ppm): $8.24(\mathrm{~d}, J=8.4$ $\mathrm{Hz}, 1 \mathrm{H}), 8.17(\mathrm{~d}, J=8.0 \mathrm{~Hz}, 1 \mathrm{H}), 7.96(\mathrm{~d}, J=8.0 \mathrm{~Hz}, 2 \mathrm{H}), 7.90-7.86(\mathrm{~m}, 1 \mathrm{H})$, 7.85-7.81 (m, 1H), 7.71-7.69 (m, 2H), $7.62(\mathrm{t}, J=7.6 \mathrm{~Hz}, 1 \mathrm{H}), 7.48(\mathrm{t}, J=7.6 \mathrm{~Hz}$, 2H), 7.40-7.38 (m, 3H); ${ }^{13} \mathrm{C}$ NMR (100 MHz, $\left.\mathrm{CDCl}_{3}, \delta \mathrm{ppm}\right): 194.0,152.8,151.3$, $142.1,139.6$, 137.2, 135.5, 134.1, 131.4, 130.5, 130.5, 129.6, 129.5, 129.4, 129.1, 128.7; HRMS (ESI): calcd. for $\mathrm{C}_{21} \mathrm{H}_{14} \mathrm{~N}_{2} \mathrm{O}_{1}+\mathrm{H}=311.1179$, found: 311.1176 .<smiles>CCOC(=O)C(=N)/C(C)=N\c1ccccc1</smiles>

\section{(Z)-Ethyl 2-diazo-3-(phenylimino)butanoate (8)}

Yellow soild; m.p. $=50-51{ }^{\circ} \mathrm{C} ;{ }^{1} \mathrm{H} \mathrm{NMR}\left(\mathrm{CDCl}_{3}, 300 \mathrm{MHz}, \delta \mathrm{ppm}\right): 7.59-7.57(\mathrm{~m}$, $3 \mathrm{H}), 7.47-7.44(\mathrm{~m}, 2 \mathrm{H}), 4.47$ (q, $J=7.6 \mathrm{~Hz}, 2 \mathrm{H}), 2.60$ (s, 3H), $1.46(\mathrm{t}, J=7.6 \mathrm{~Hz}$, $3 \mathrm{H}) ;{ }^{13} \mathrm{C}$ NMR (75 MHz, $\left.\mathrm{CDCl}_{3}, \delta \mathrm{ppm}\right): 161.7,138.8,136.6,135.4,130.0,129.6$, 125.3, 61.0, 14.3, 9.9; HRMS (ESI): calcd. for $\mathrm{C}_{12} \mathrm{H}_{13} \mathrm{~N}_{3} \mathrm{O}_{2}+\mathrm{H}=232.1081$, found: 232.1079 .<smiles>c1ccc(-c2cnc3ccccc3n2)cc1</smiles>

\section{2-Phenylquinoxaline $(9)^{9}$}

${ }^{1} \mathrm{H}$ NMR $\left(\mathrm{CDCl}_{3}, 400 \mathrm{MHz}, \delta \mathrm{ppm}\right): 9.34(\mathrm{~s}, 1 \mathrm{H}), 8.21-8.13(\mathrm{~m}, 4 \mathrm{H}), 7.82-7.74(\mathrm{~m}$,

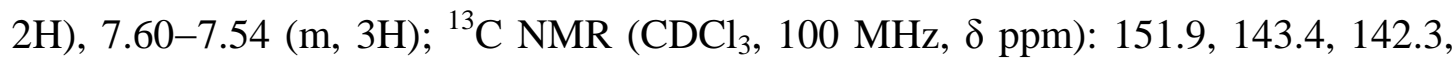
$141.5,136.7,130.3,130.2,129.6,129.6,129.2,129.1,127.5$. ESI-MS: $\mathrm{M}+\mathrm{H}^{+}$: 207.1280 .<smiles>N#CC(=Nc1ccccc1)c1ccccc1</smiles>

\section{(Z)-N-phenylbenzimidoyl cyanide $(\mathbf{1 0})^{10}$}

${ }^{1} \mathrm{H} \mathrm{NMR}\left(\mathrm{CDCl}_{3}, 400 \mathrm{MHz}, \delta \mathrm{ppm}\right): 8.16(\mathrm{~d}, J=7.2 \mathrm{~Hz}, 2 \mathrm{H}), 7.62-7.58(\mathrm{~m}, 1 \mathrm{H})$, $7.54(\mathrm{t}, J=7.6 \mathrm{~Hz}, 2 \mathrm{H}), 7.47(\mathrm{t}, J=8.0 \mathrm{~Hz}, 2 \mathrm{H}), 7.32(\mathrm{t}, J=7.6 \mathrm{~Hz}, 1 \mathrm{H}), 7.20-7.18(\mathrm{~m}$, $2 \mathrm{H}) ;{ }^{13} \mathrm{C} \mathrm{NMR}\left(\mathrm{CDCl}_{3}, 100 \mathrm{MHz}, \delta \mathrm{ppm}\right): 149.1,139.8,133.6,132.9,129.3,129.0$, 
128.2, 127.3, 120.3, 110.9. ESI-MS: $\mathrm{M}+\mathrm{H}^{+}: 207.1256$.

\section{References}

1. Zhang, Z. H.; Yin, L.; Wang, Y. M. Adv. Synth.Catal. 2006, 348, 184.

2. Yu, W.; Du, Y.; Zhao K. Org. Lett. 2009, 11, 2417.

3. Barluenga, J.; Jiménez-Aquino, A.; Aznar, F.; Valdés, C. J. Am. Chem. Soc. 2009, $131,4031$.

4. He, Z.; Liu, W.; Li, Z. Chem.-Asian J. 2011, 6, 1340.

5. Cao, H.; Wang, X.; Jiang, H. Chem.-Eur. J. 2008, 14, 11623.

6. Kuo, F.-C.; Lee, H.-Z.; Juang J.-P.; Lin Y.-T.; Wu T.-S. J. Med. Chem. 1993, 36, 1146.

7. Pidathala, I.; Amewu, R.; Pacorel, B.; Nixon, G. L. J. Med. Chem. 2012, 55, 1831.

8. Jia, Z.; Nagano, T.; Li, X.; Chan, A. S. C. Eur. J. Org. Chem. 2013, 858.

9. Jeganathan, M.; Dhakshinamoorthy, A.; Pitchumani, K. Tetrahedron Lett. 2014, $55,1616$.

10. Fontaine, P.; Chiaroni, A.; Masson, G.; Zhu, J. Org. Lett. 2008, 10, 1509. 
Copies of ${ }^{1} \mathrm{H}$ NMR and ${ }^{13} \mathrm{C}$ NMR spectra of the substrates $1 a$

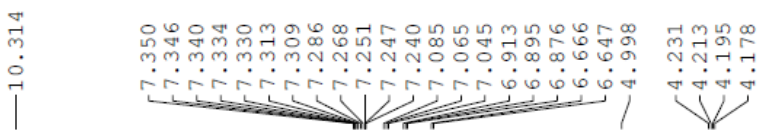
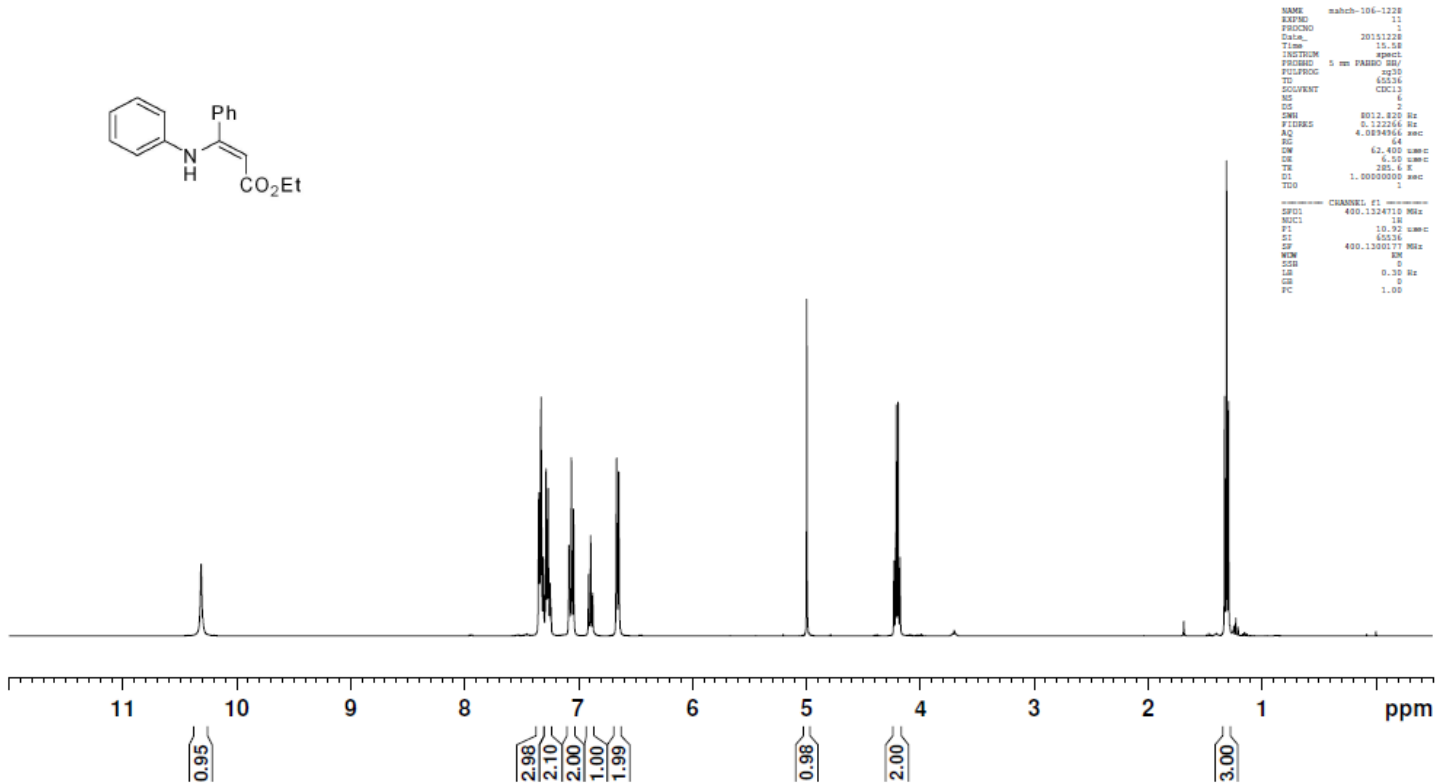<smiles>CCOC(=O)/C=C(\Nc1ccccc1)c1ccccc1</smiles>
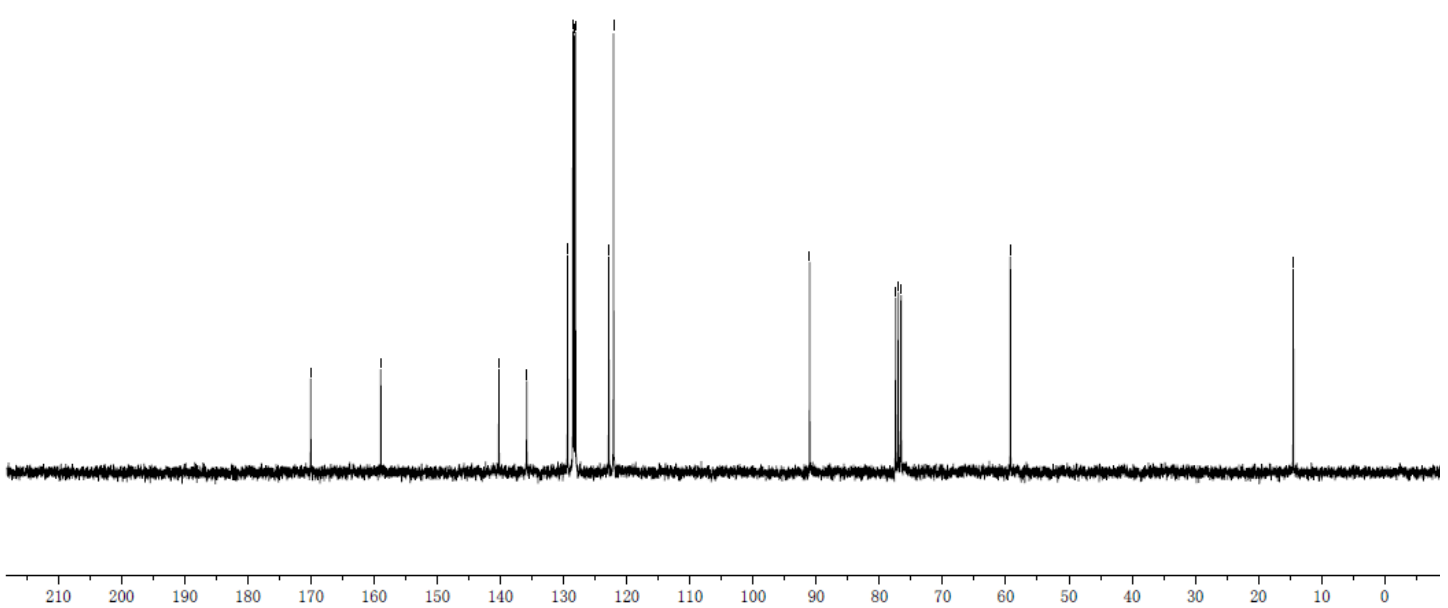
1b

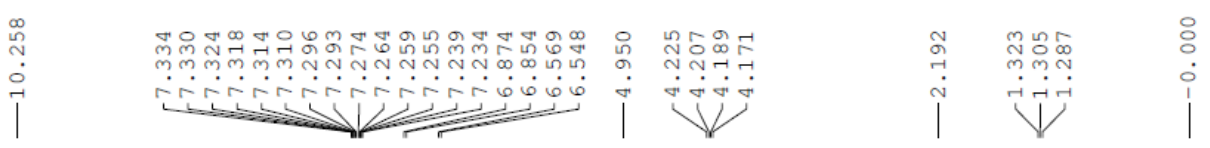<smiles>CCOC(=CC(=O)Nc1ccc(N)cc1)COC</smiles>

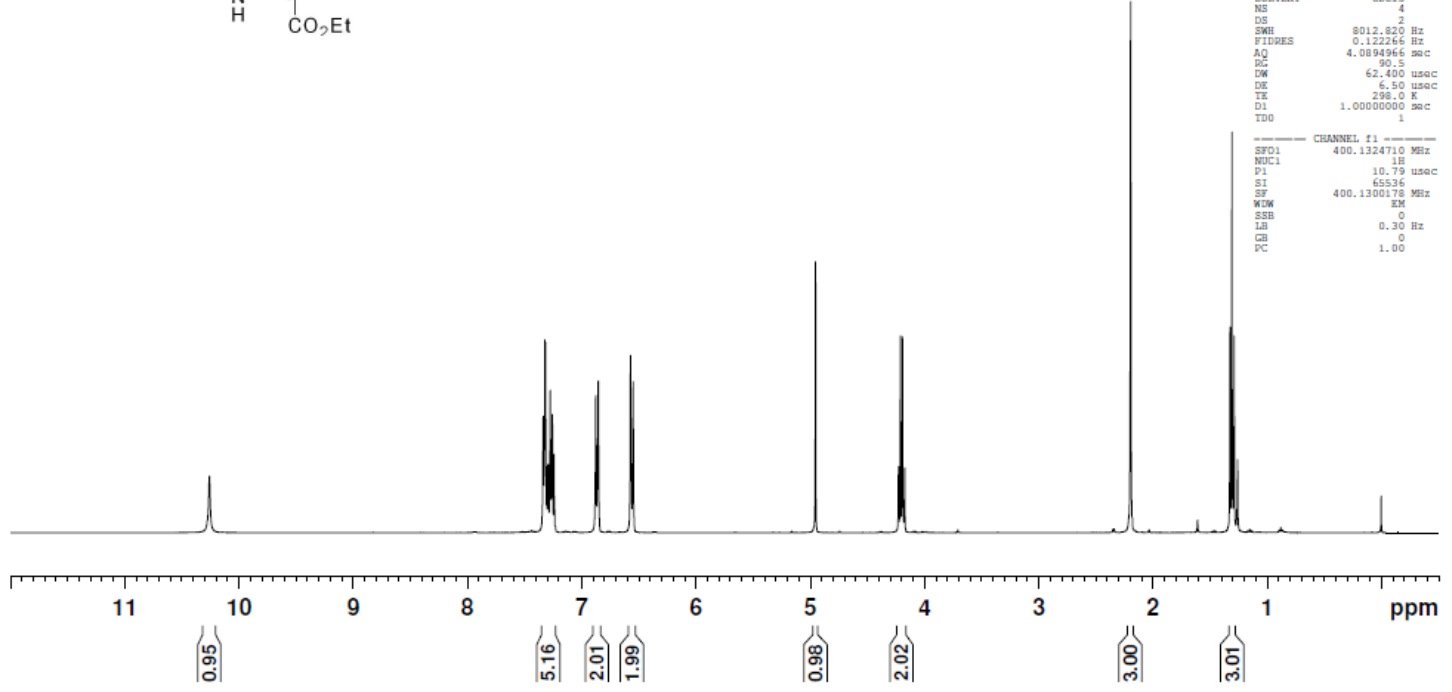

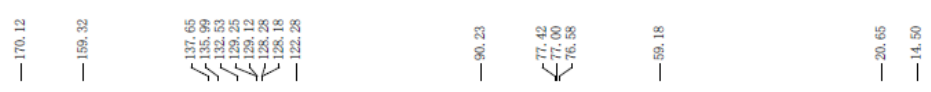
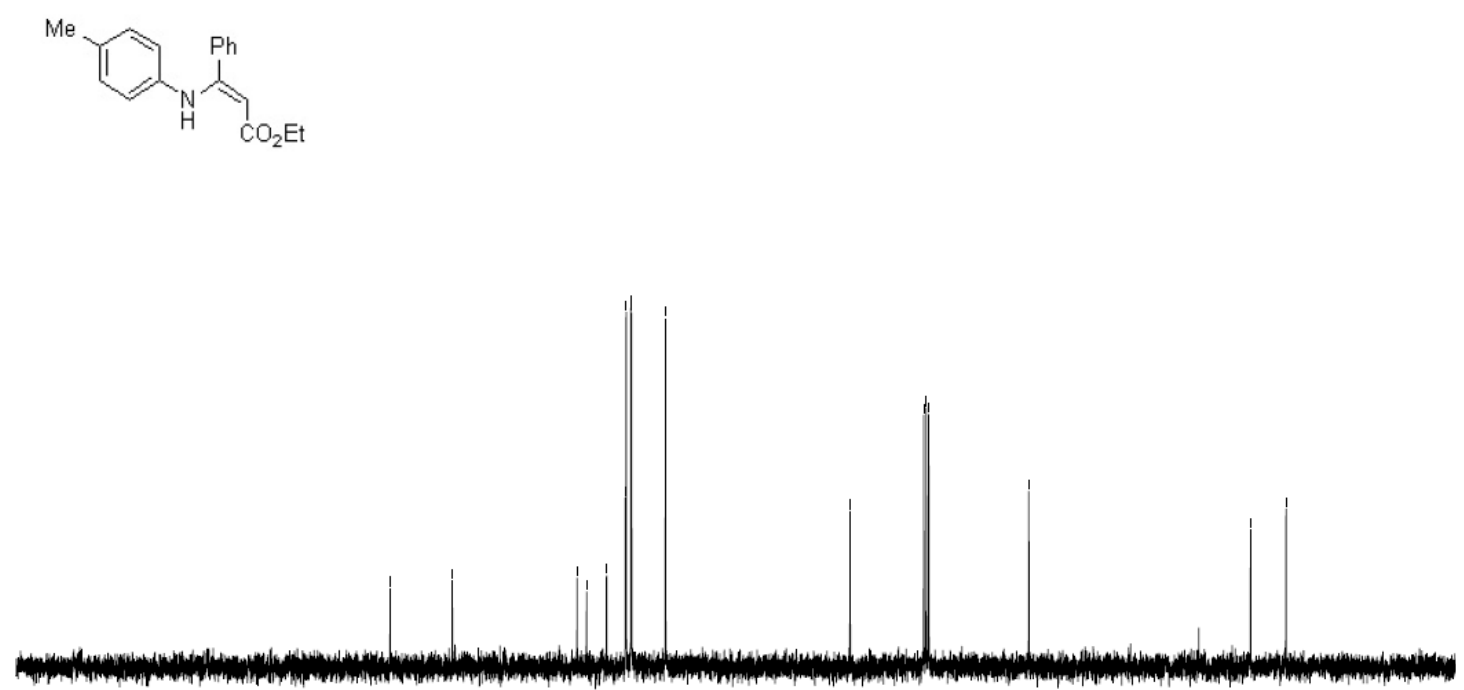

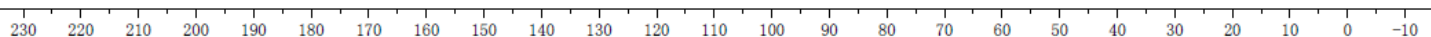


1c

|

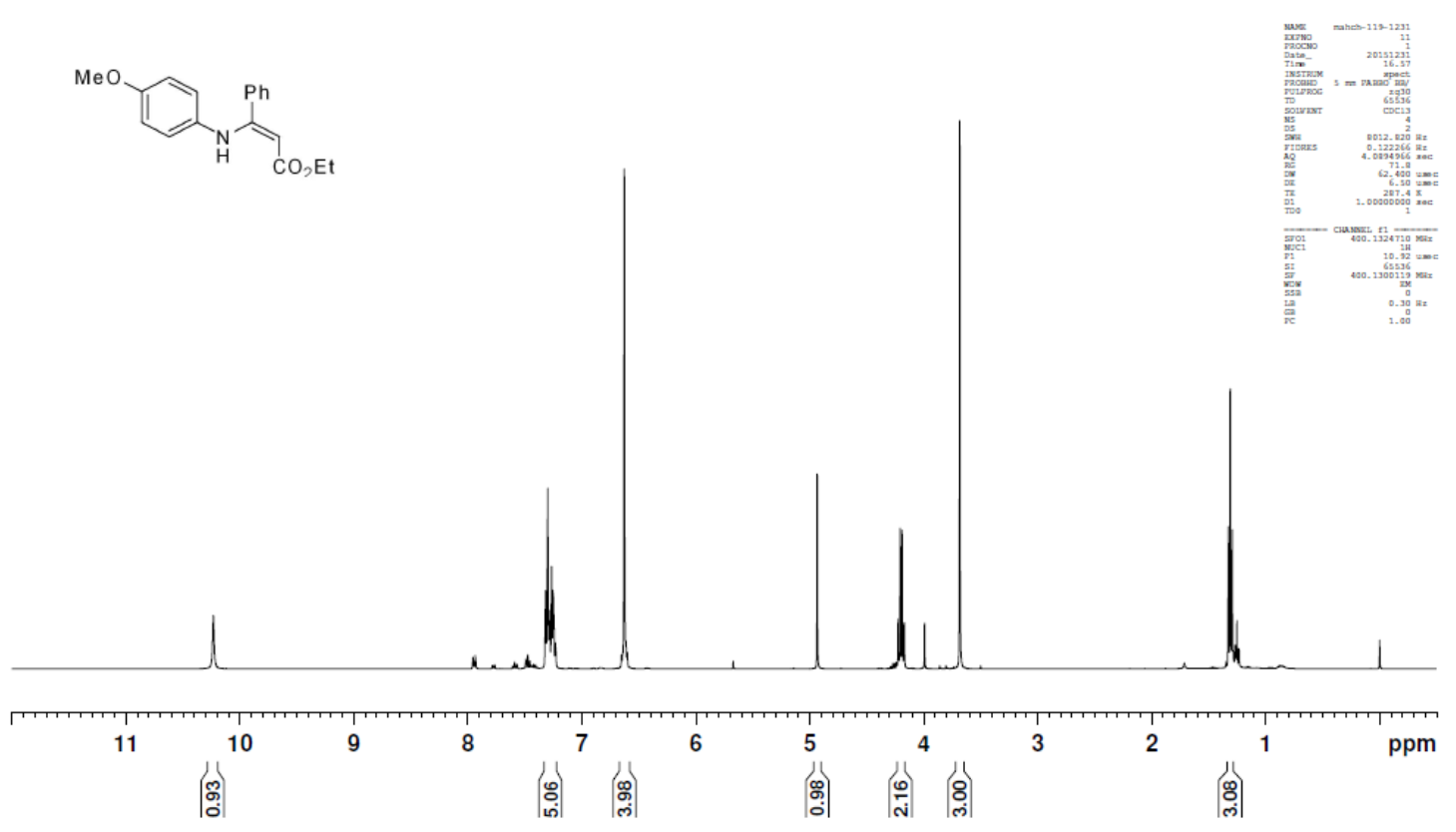

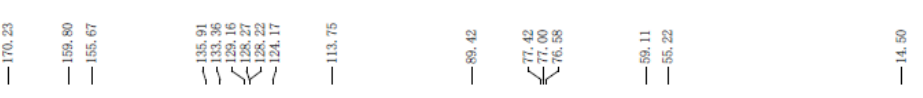

MeO

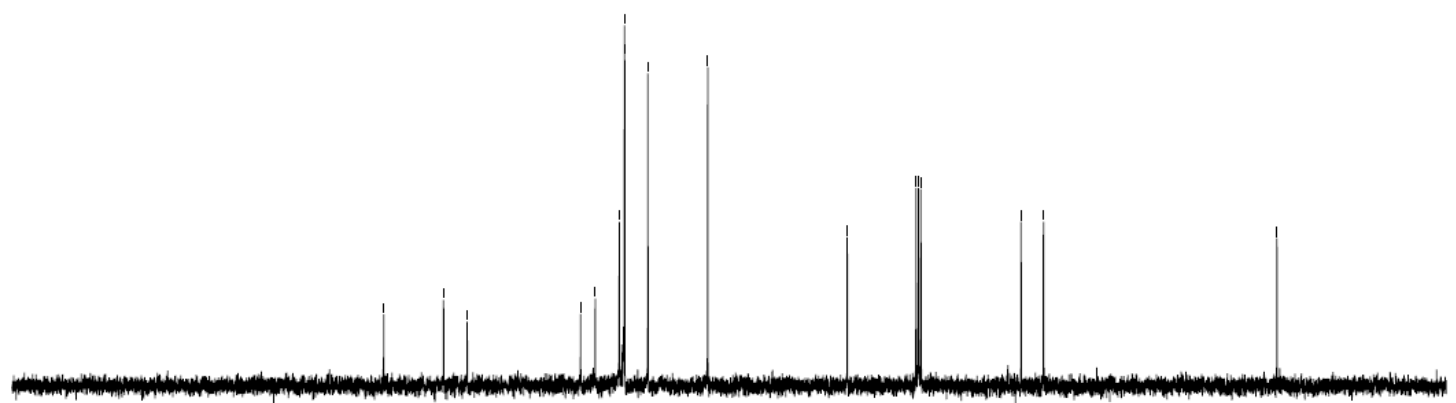

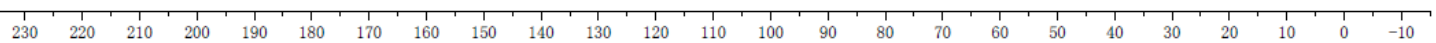


1d

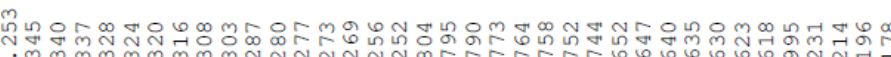

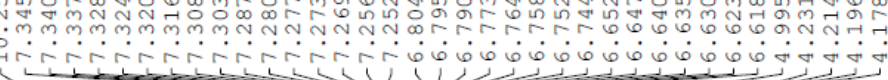

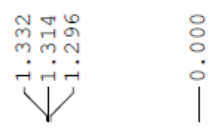

(1)

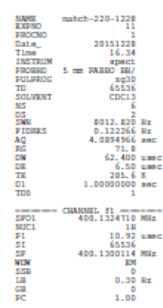

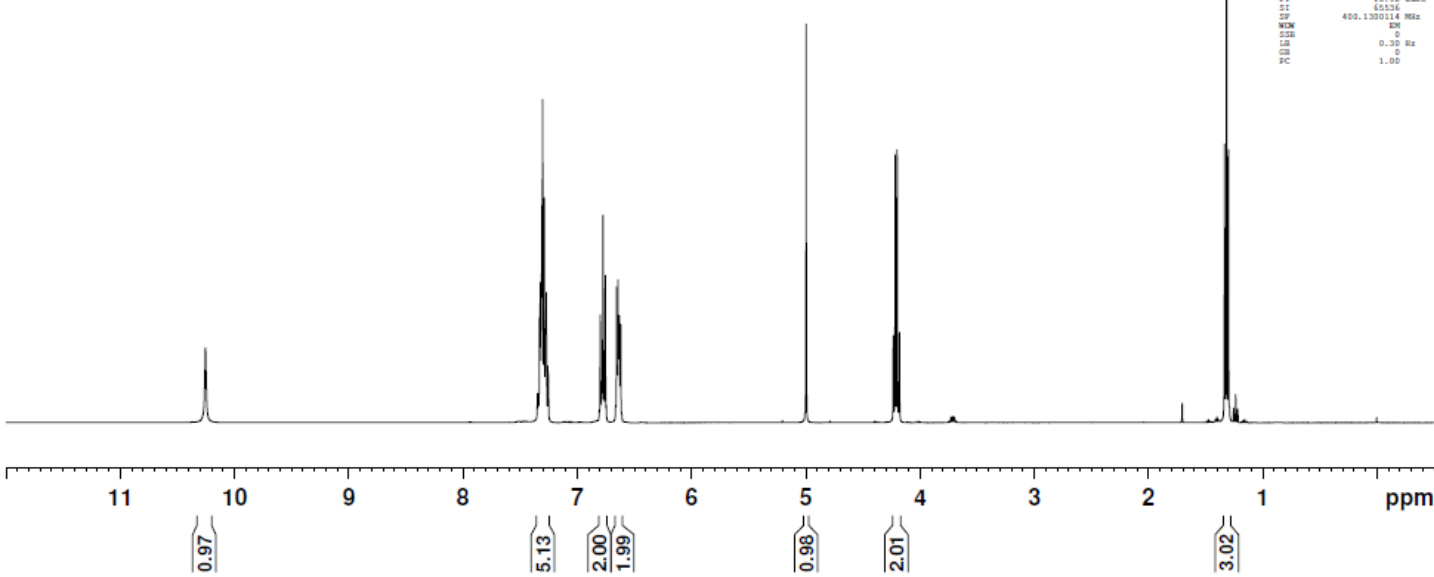

| V|

$\overbrace{\mathrm{H}}^{\mathrm{Ph}} \overbrace{\mathrm{CO}, \mathrm{Et}}^{\mathrm{Ph}}$

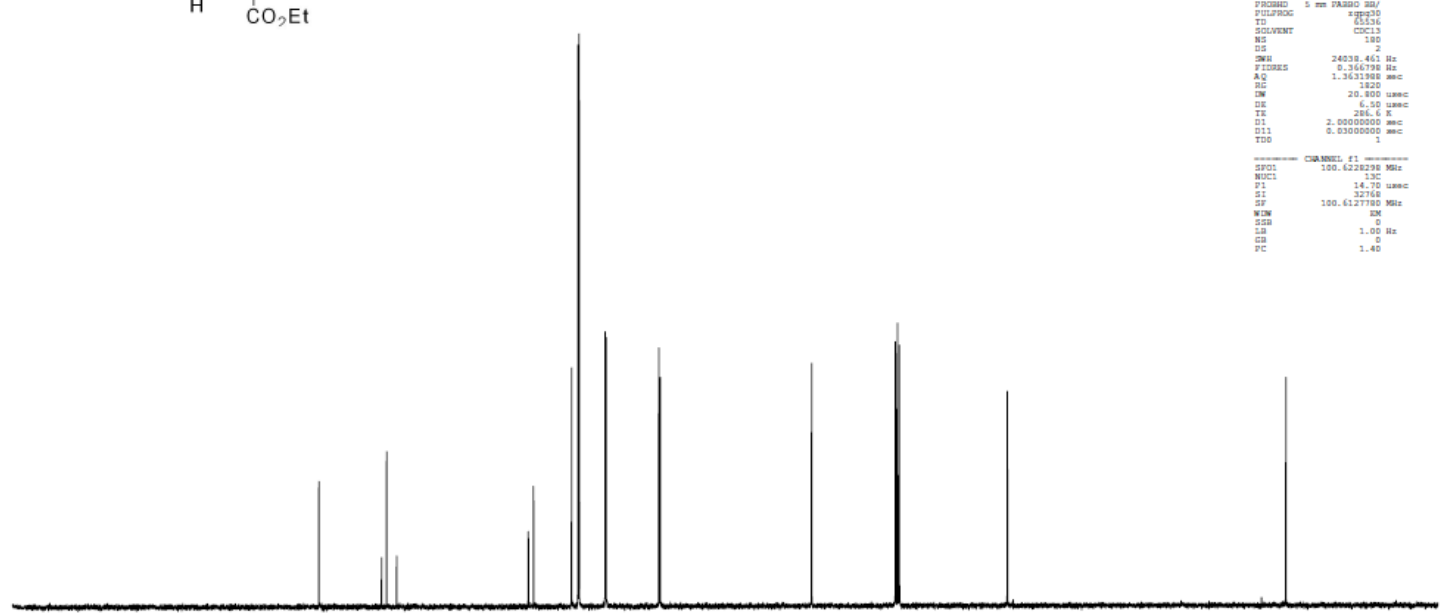

$\begin{array}{llllllllllllllllllllll}210 & 200 & 190 & 180 & 170 & 160 & 150 & 140 & 130 & 120 & 110 & 100 & 90 & 80 & 70 & 60 & 50 & 40 & 30 & 20 & 10 & \mathrm{ppm}\end{array}$ 
$1 e$

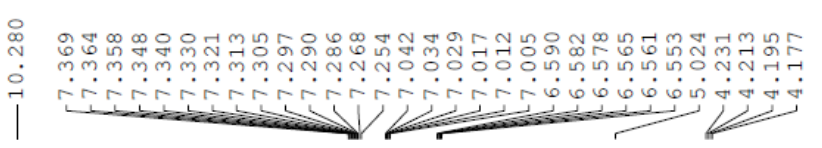

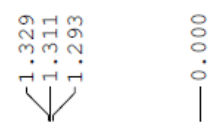

政
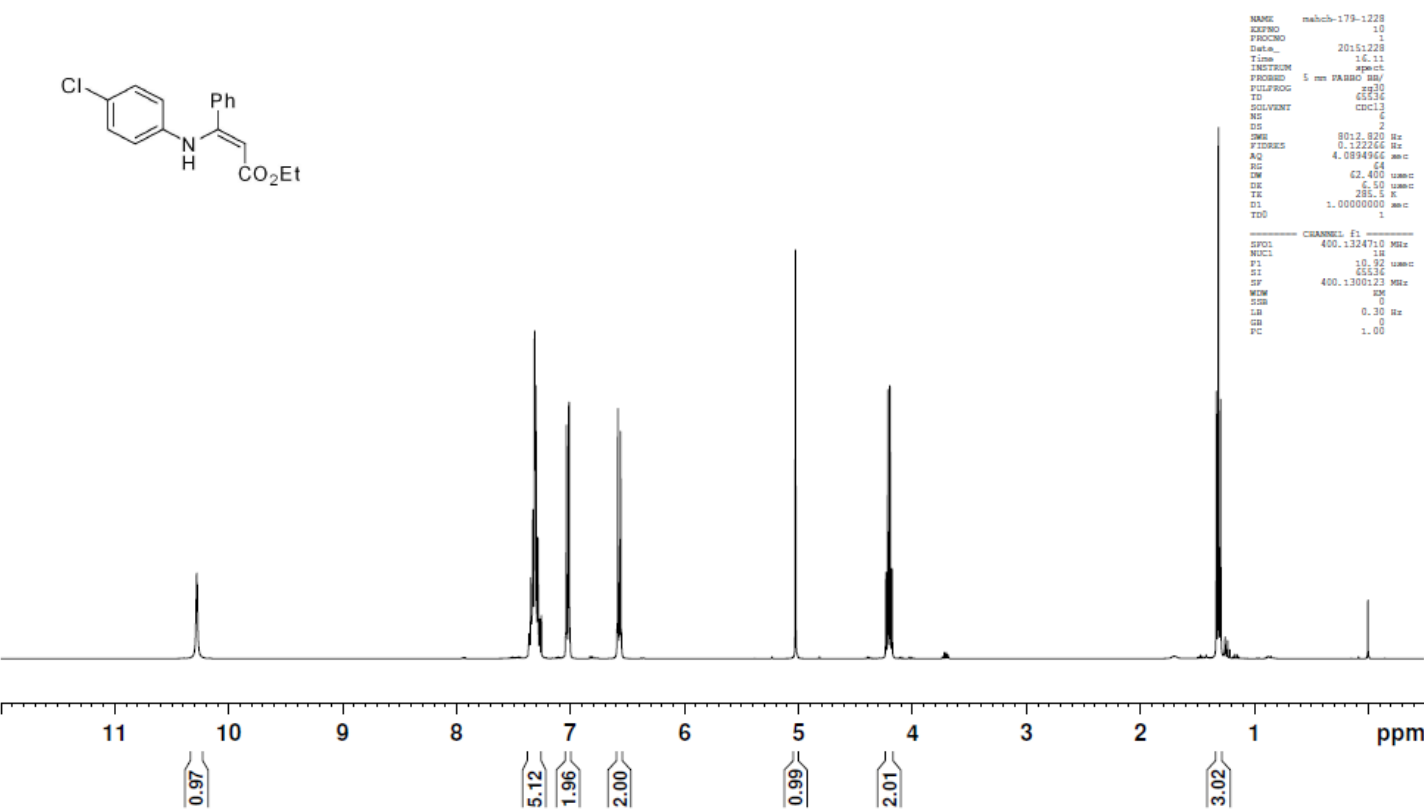

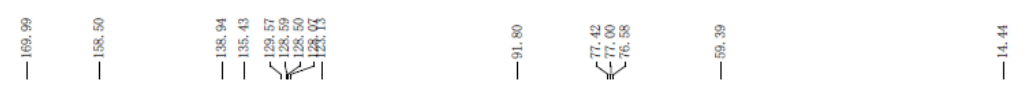

CO

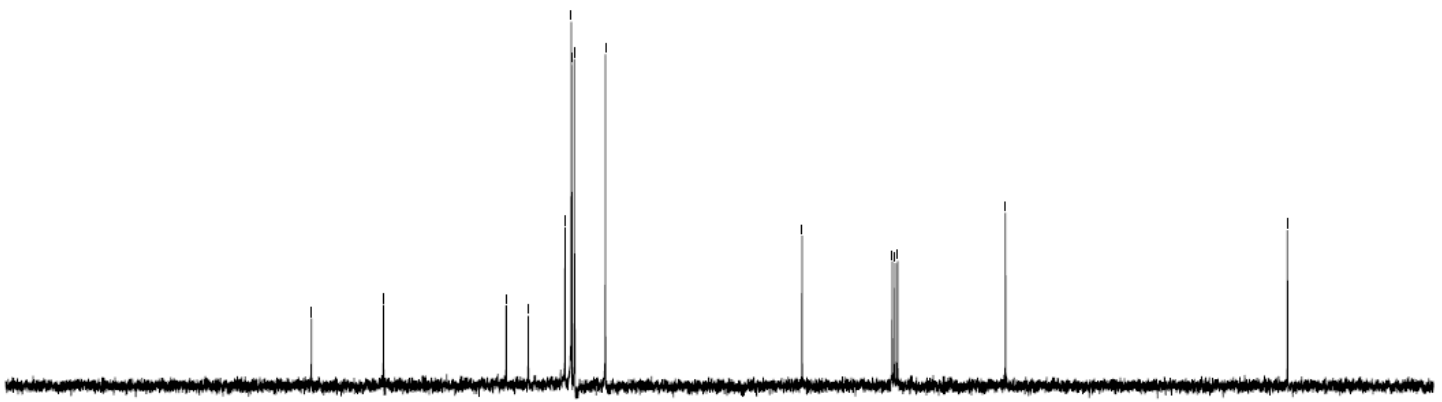


1f

|

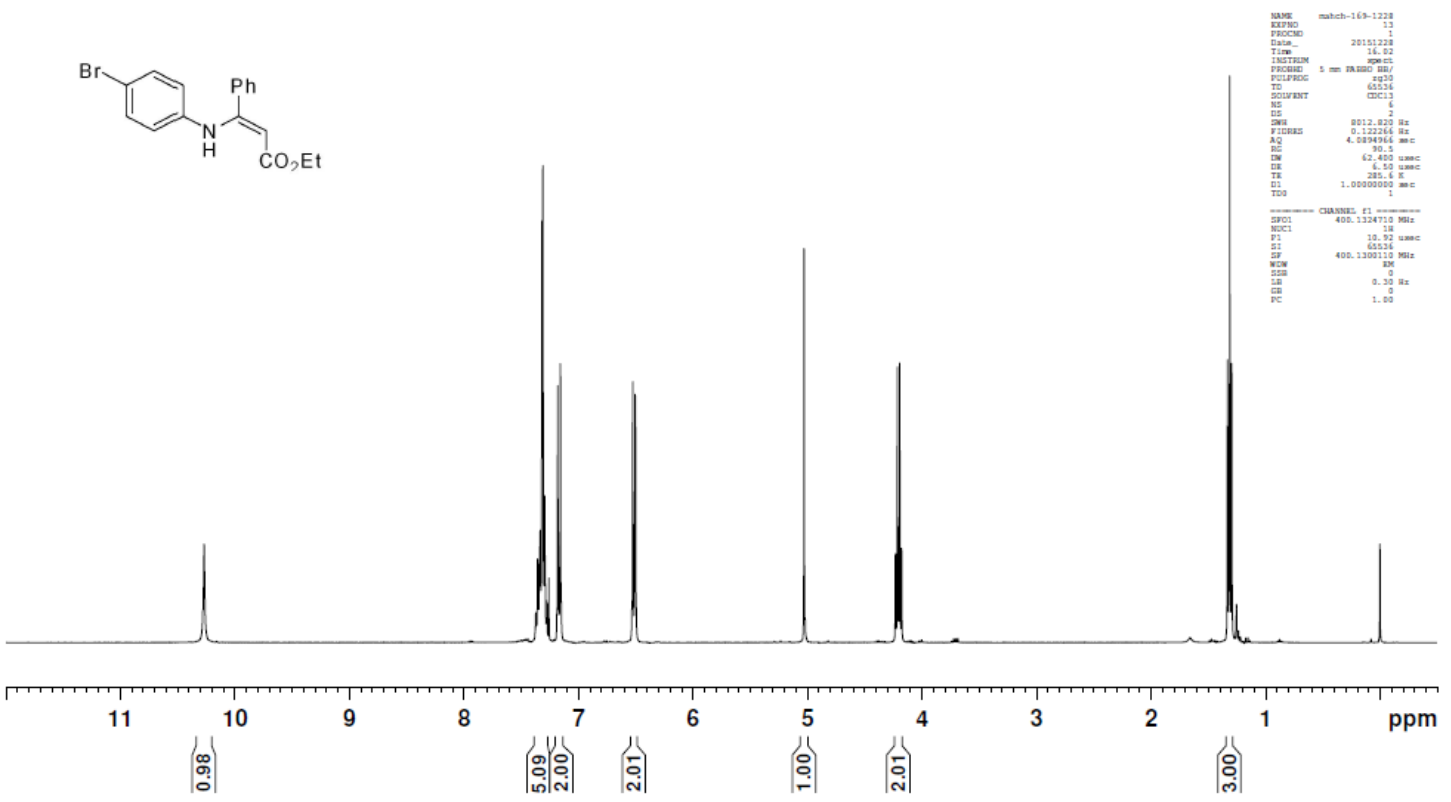

11 留

的

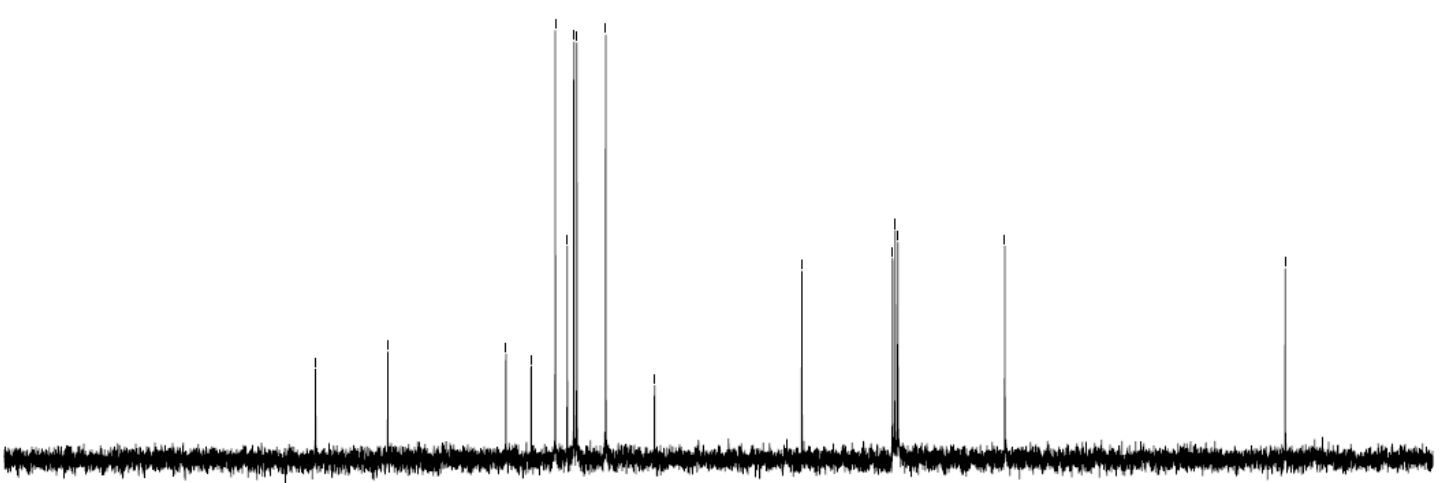

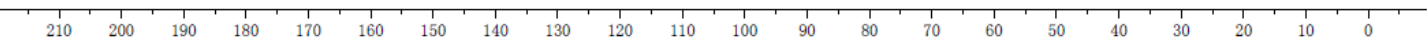


$1 \mathrm{~g}$

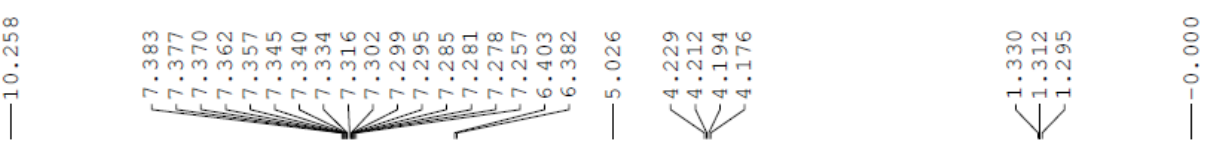

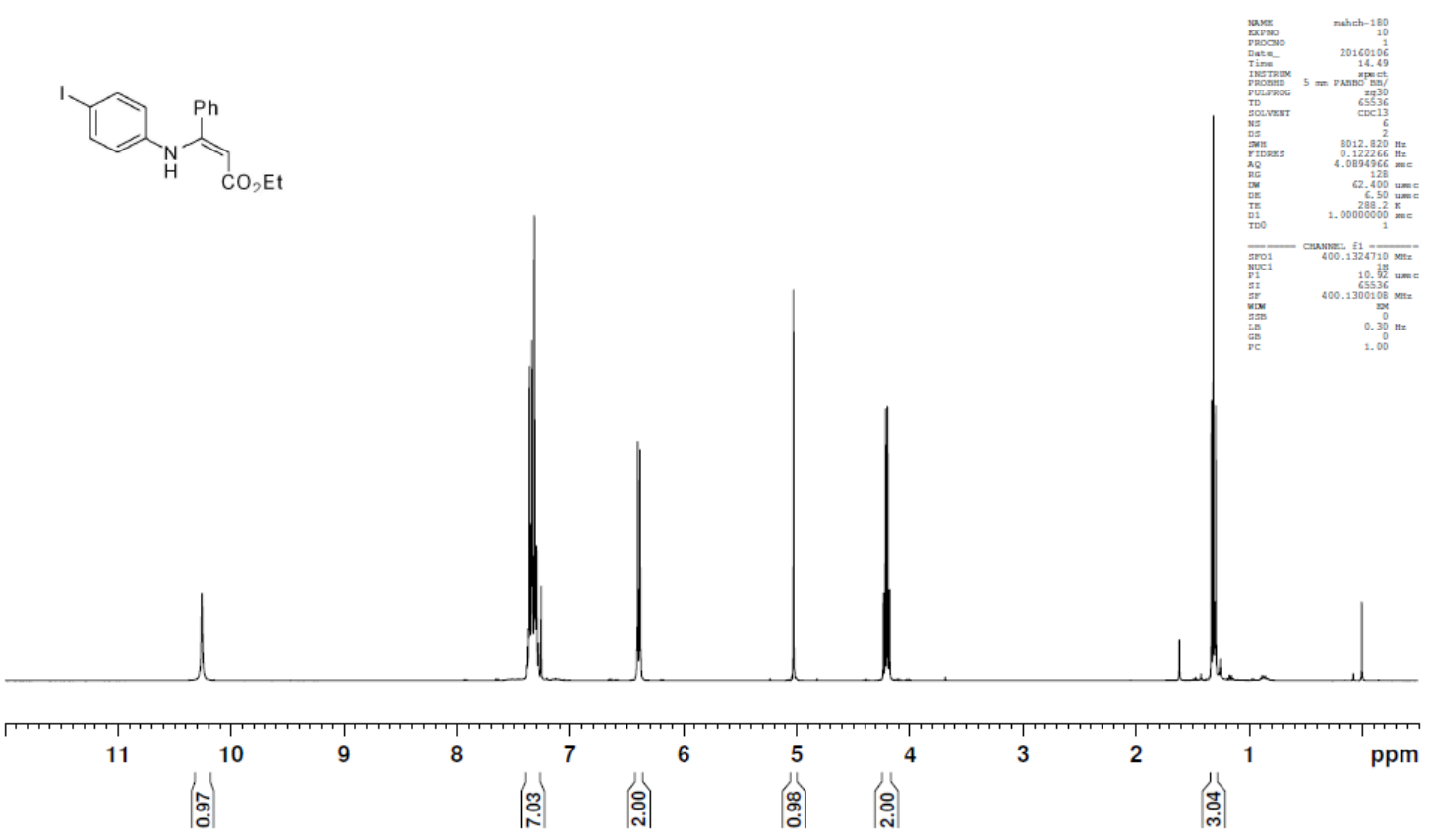

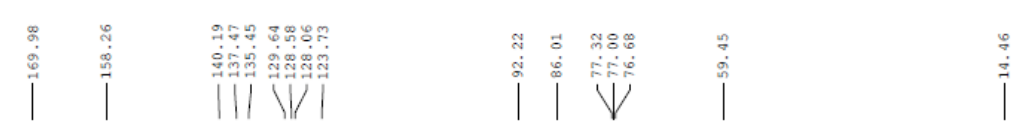

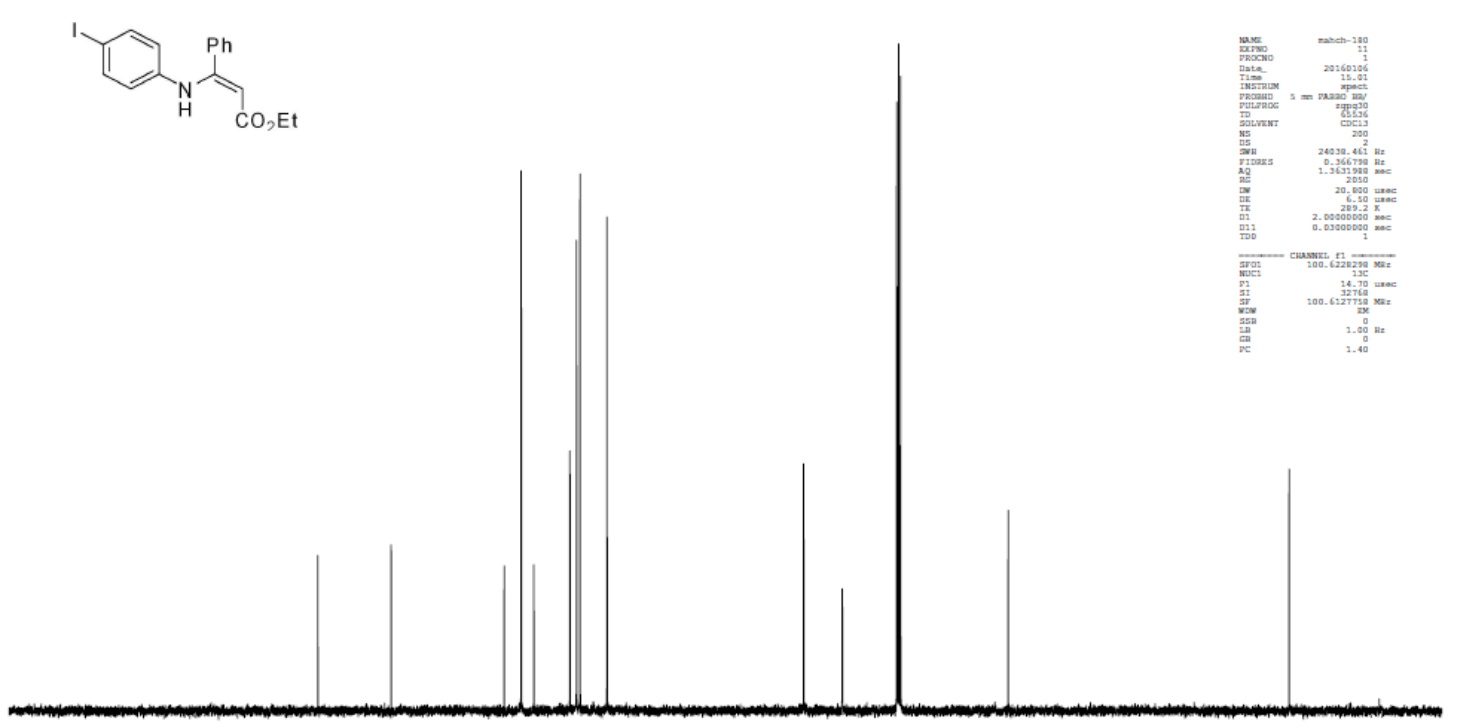

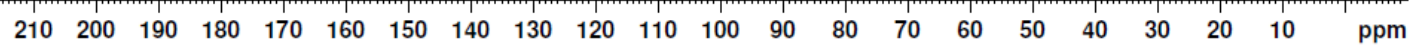


1h

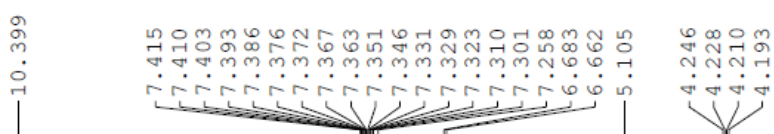

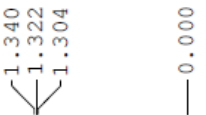

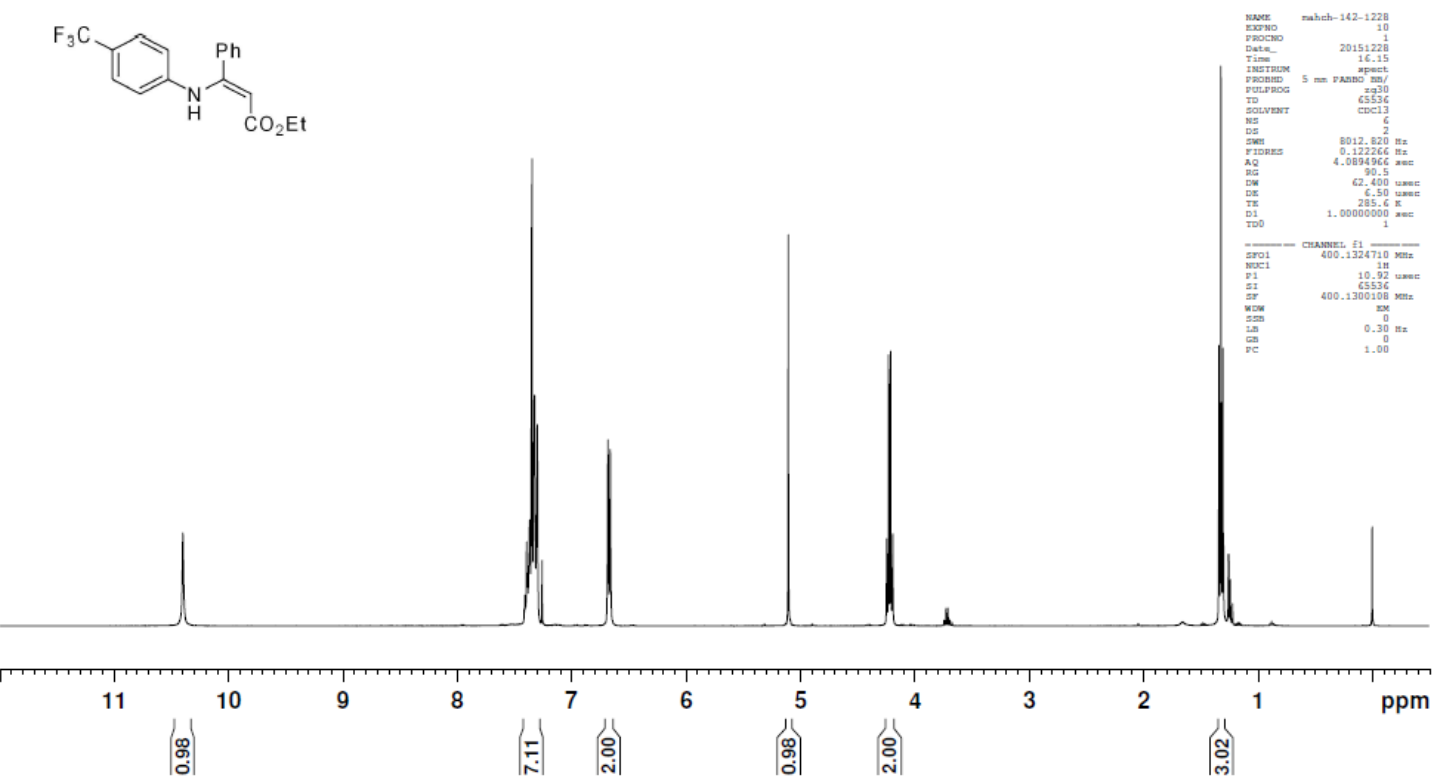

|<smiles>CC=CCC</smiles><smiles>CCOC(=O)Nc1ccc(C(F)(F)F)cc1</smiles>

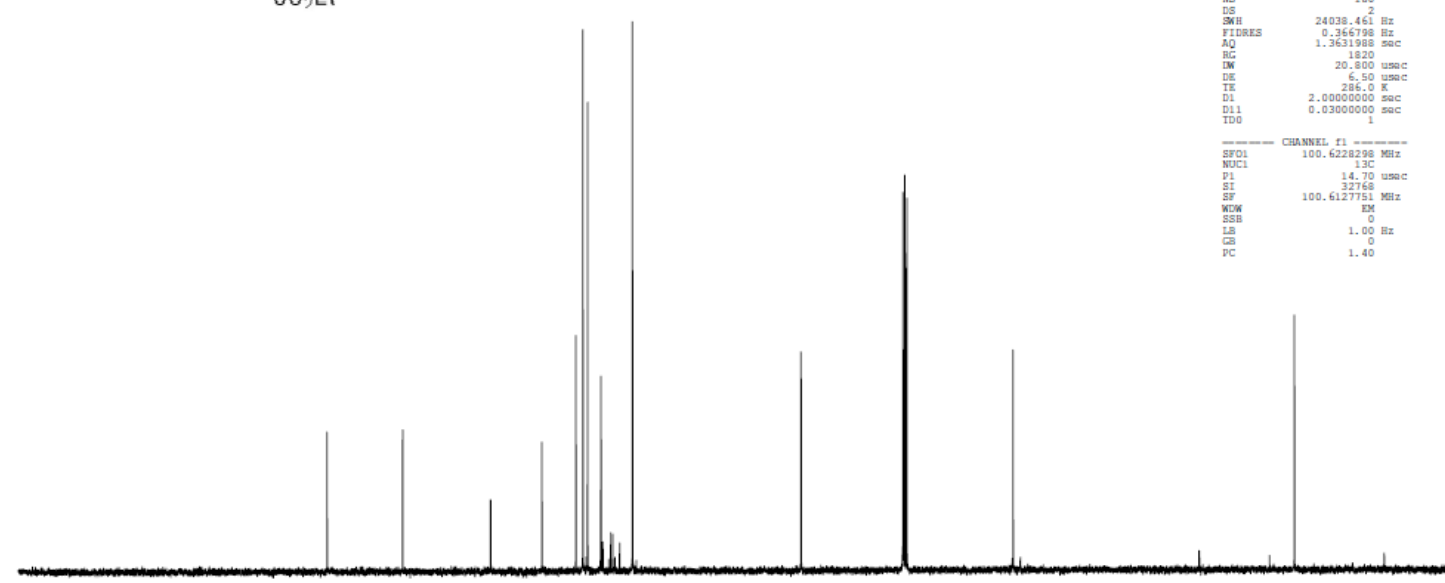

$\begin{array}{llllllllllllllllllllll}210 & 200 & 190 & 180 & 170 & 160 & 150 & 140 & 130 & 120 & 110 & 100 & 90 & 80 & 70 & 60 & 50 & 40 & 30 & 20 & 10 & \mathrm{ppm}\end{array}$ 
1i

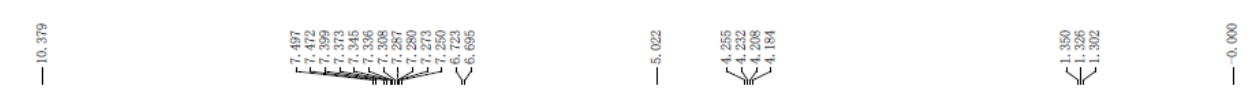

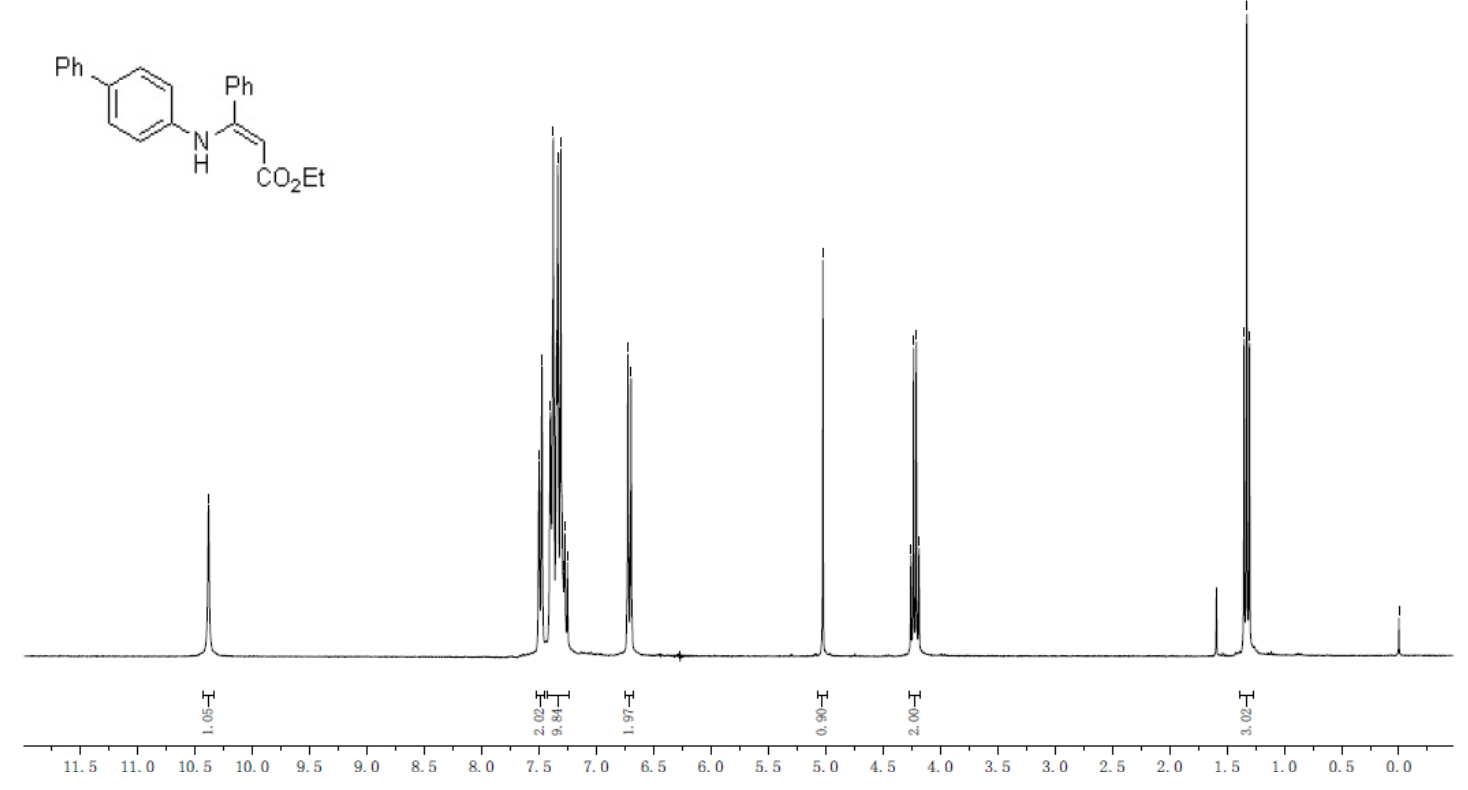

(chet
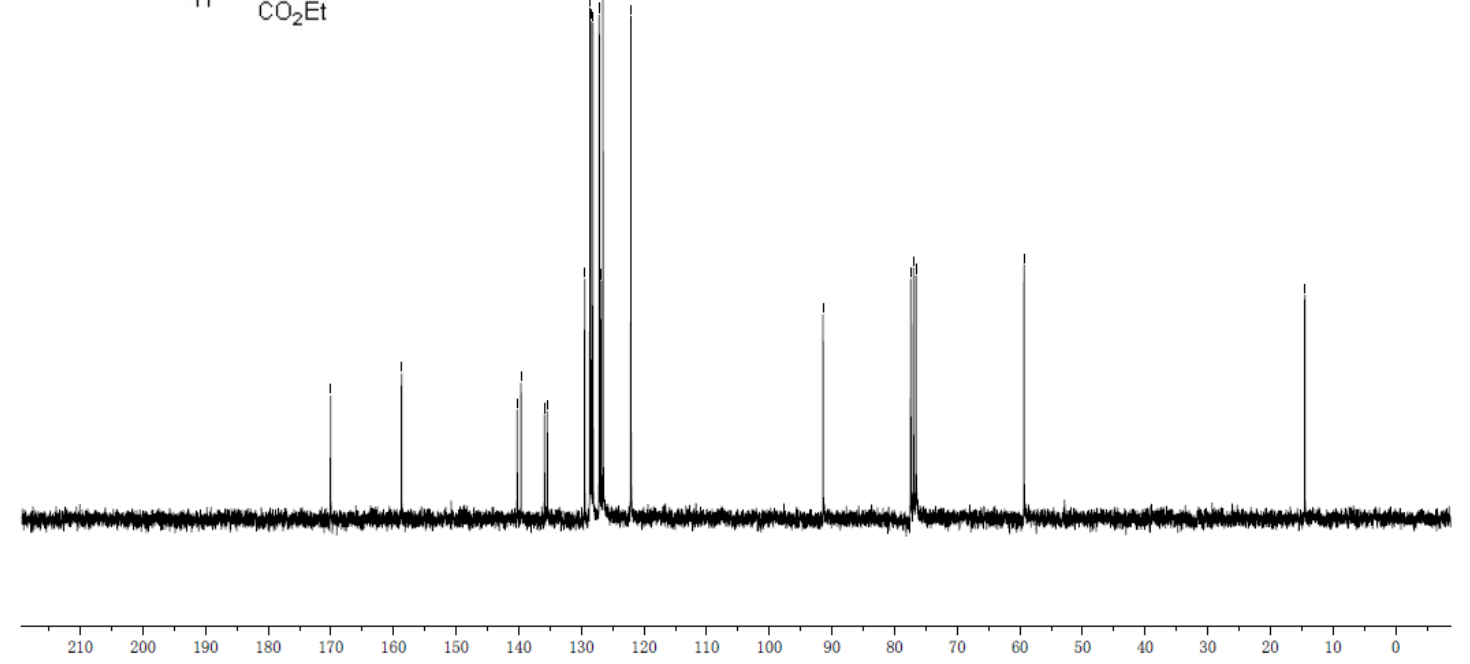
$\mathbf{1 j}$

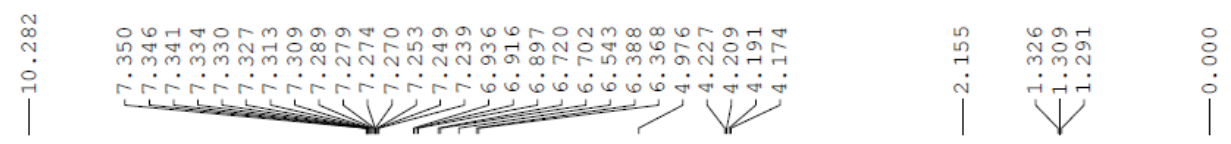

$\overbrace{\mathrm{H}} \overbrace{\mathrm{CO}_{2} \mathrm{Et}}^{\mathrm{Ph}}$

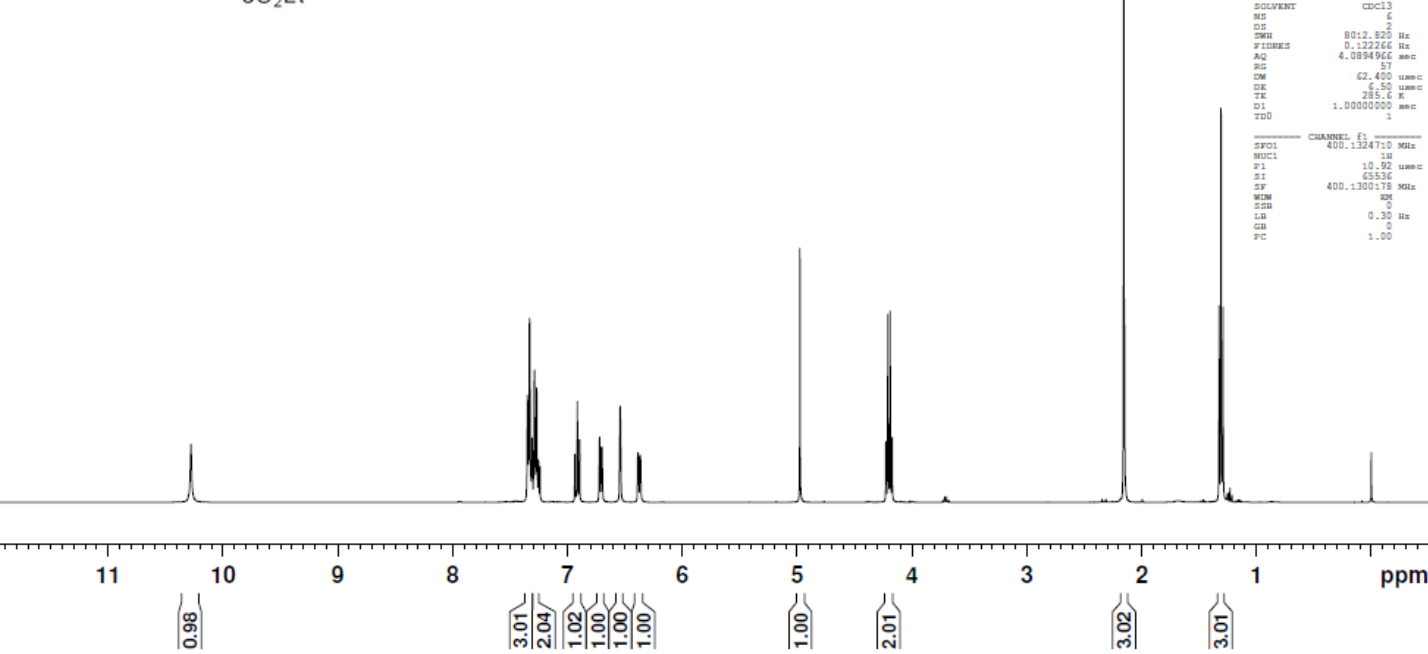

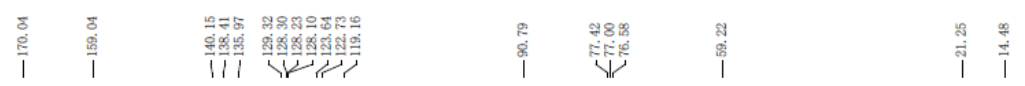

$\overbrace{\mathrm{H}}^{\mathrm{Ph}}$

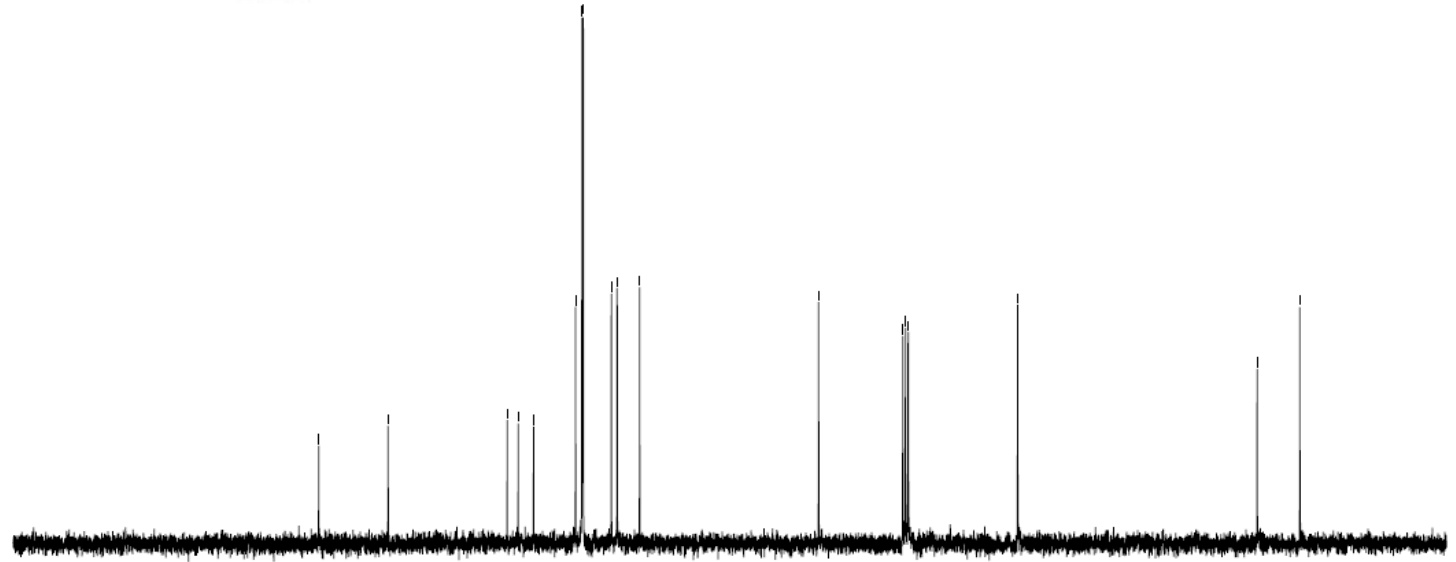

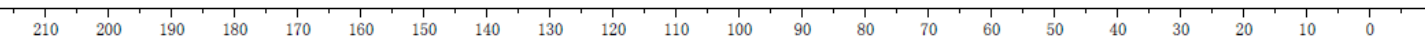


$1 k$

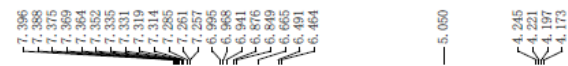

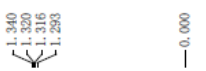

(N)

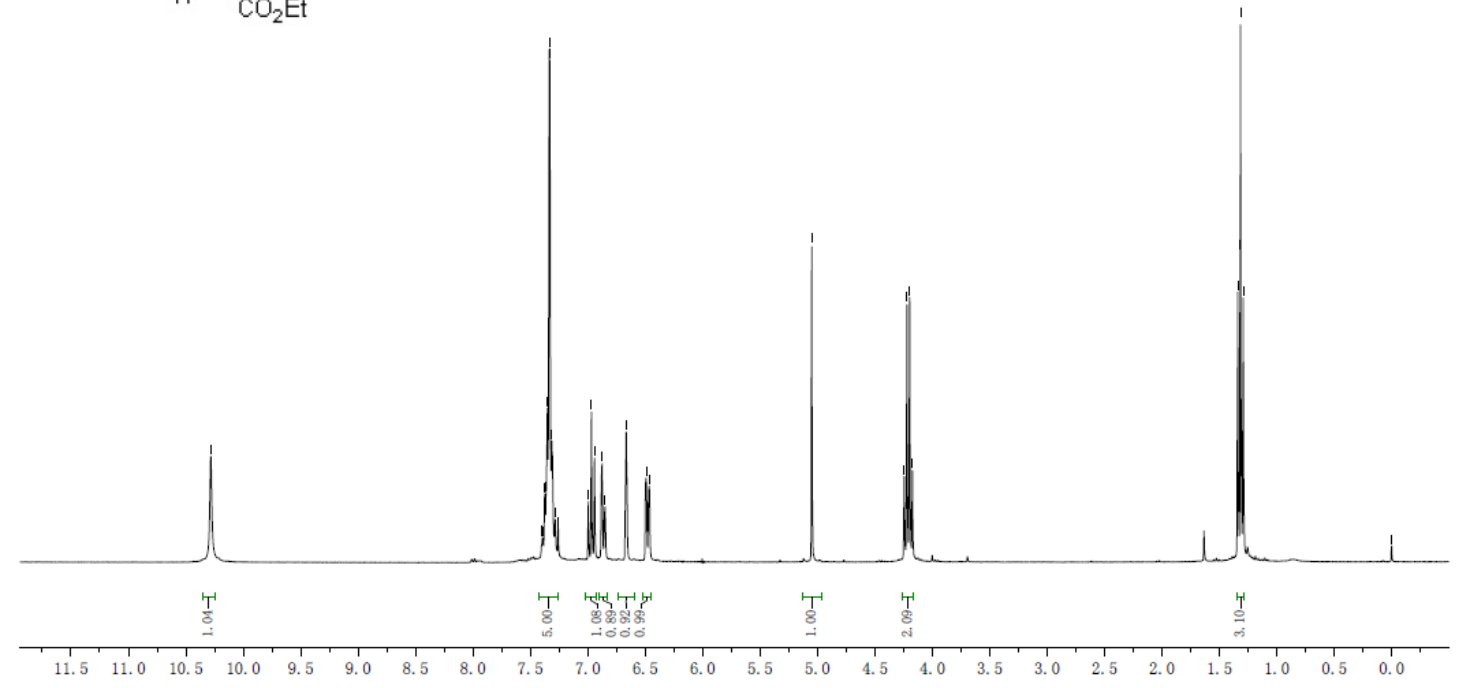

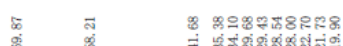

(1)

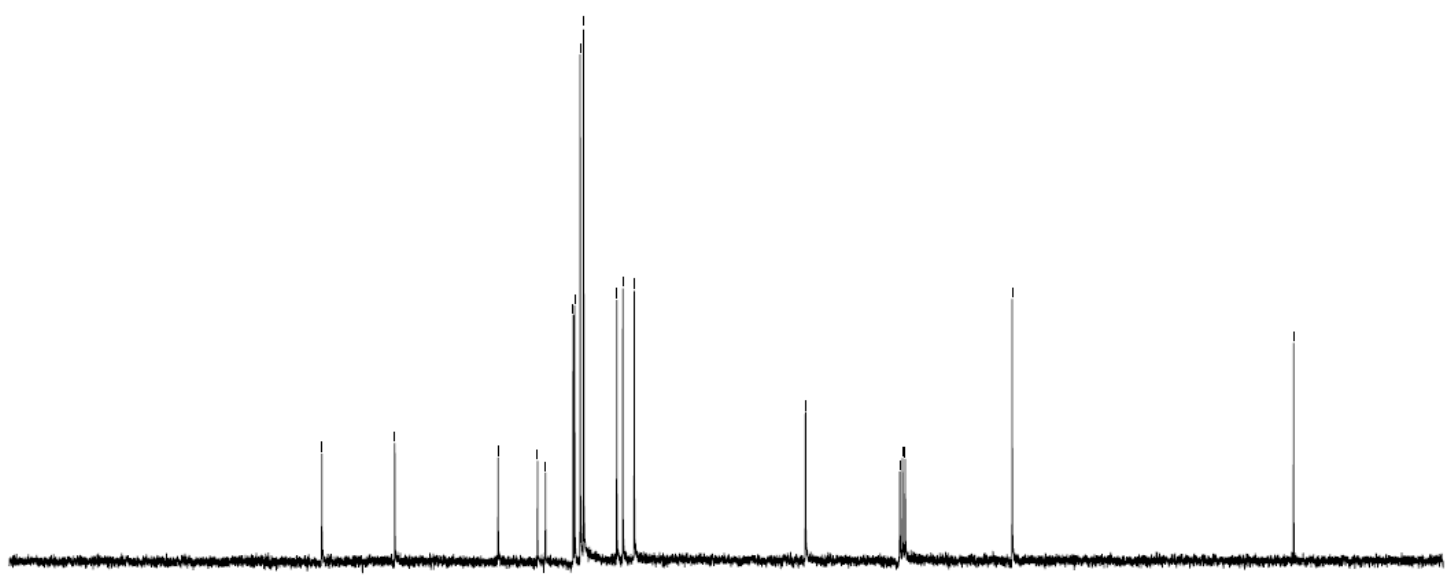


11

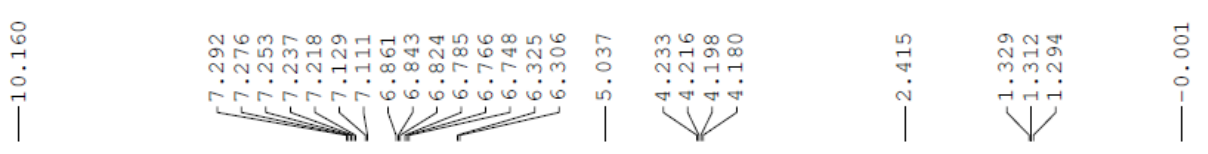

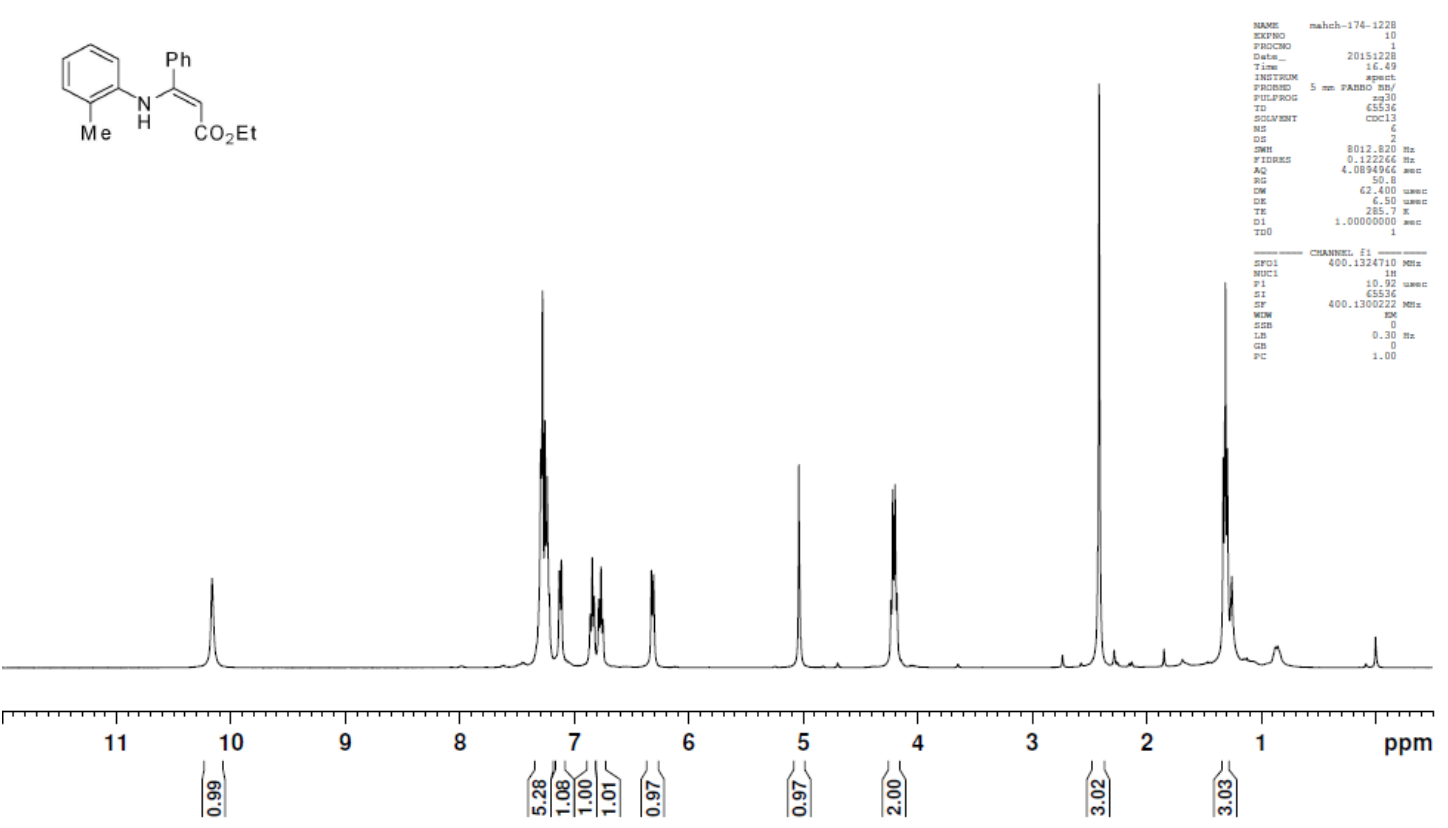

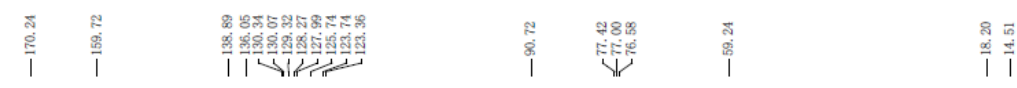

Me

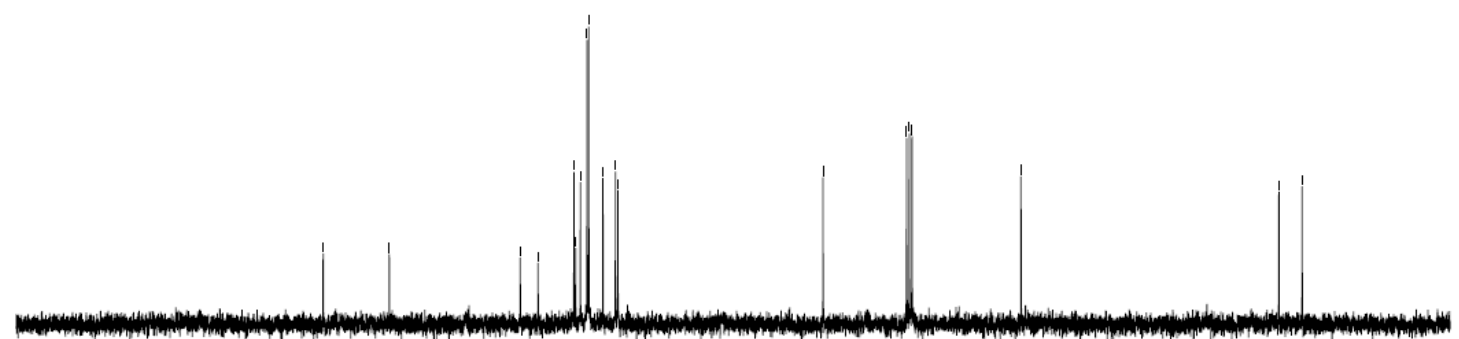

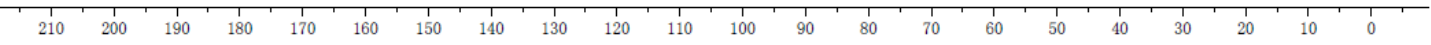


$1 \mathrm{~m}$

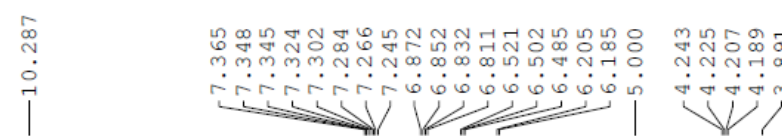

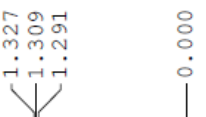

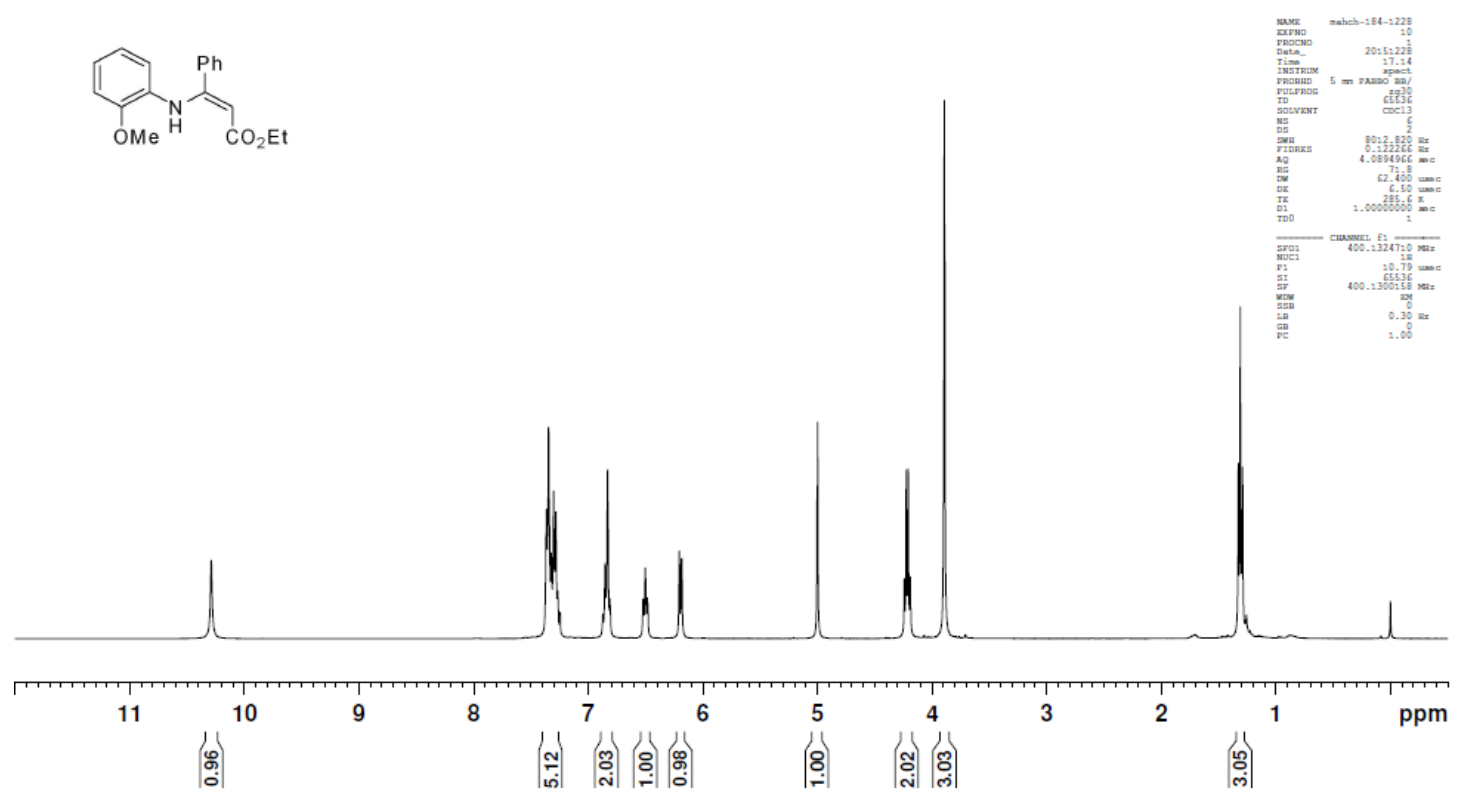

|
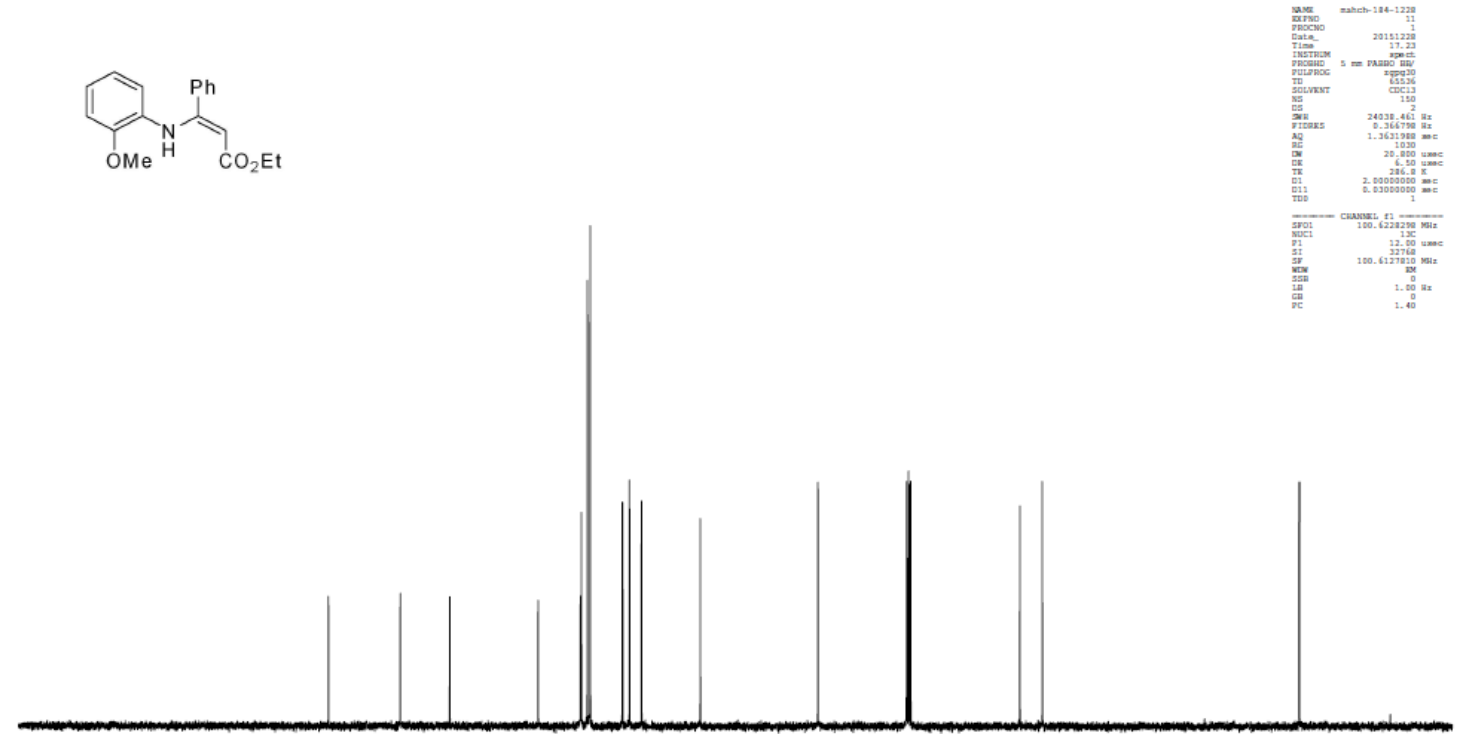

$\begin{array}{llllllllllllllllllllll}210 & 200 & 190 & 180 & 170 & 160 & 150 & 140 & 130 & 120 & 110 & 100 & 90 & 80 & 70 & 60 & 50 & 40 & 30 & 20 & 10 & \mathrm{ppm}\end{array}$ 
1n

$\frac{\sqrt{5}}{1}$

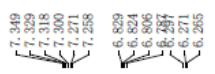

i.

ฟ

8
0
0<smiles>CCOC(=O)/C=C(\Nc1ccccc1Cl)c1ccccc1</smiles>

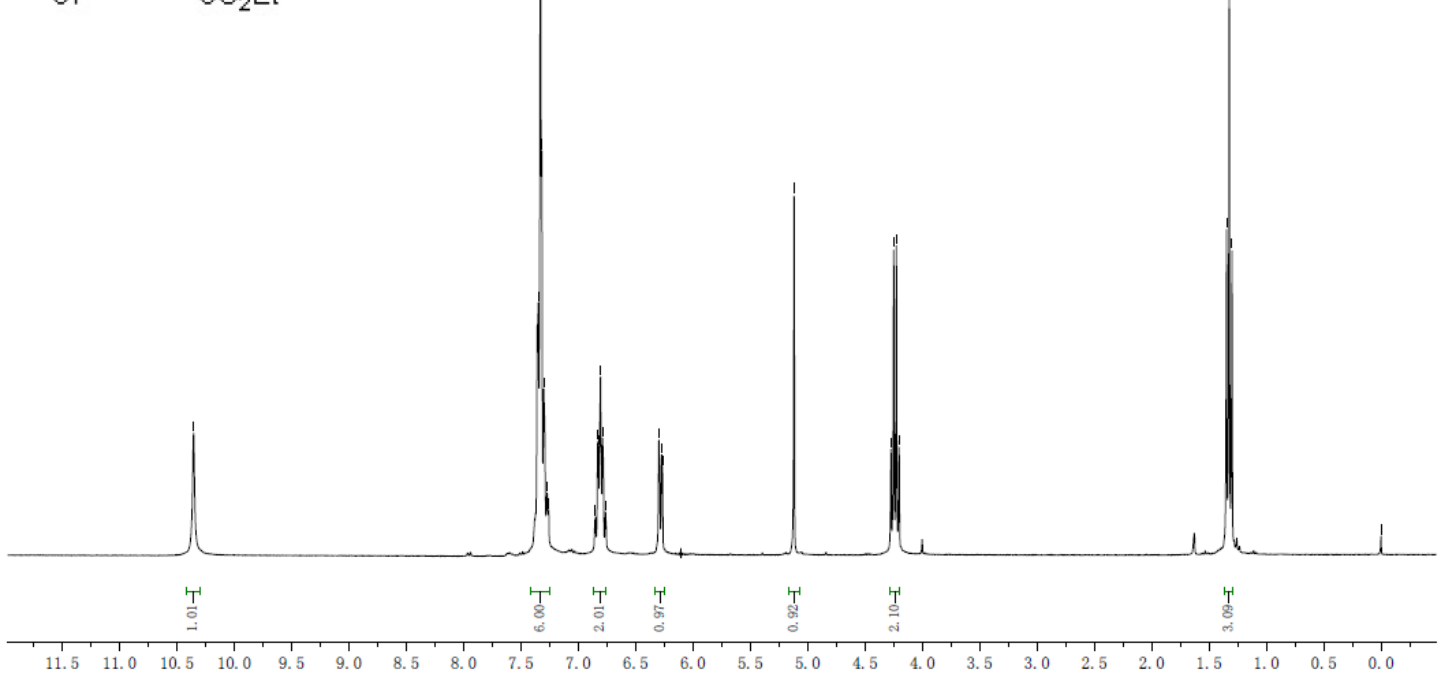

$\begin{array}{ll}5 & 8 \\ 1 & 1\end{array}$

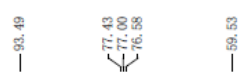

$\stackrel{g}{\stackrel{m}{i}}$
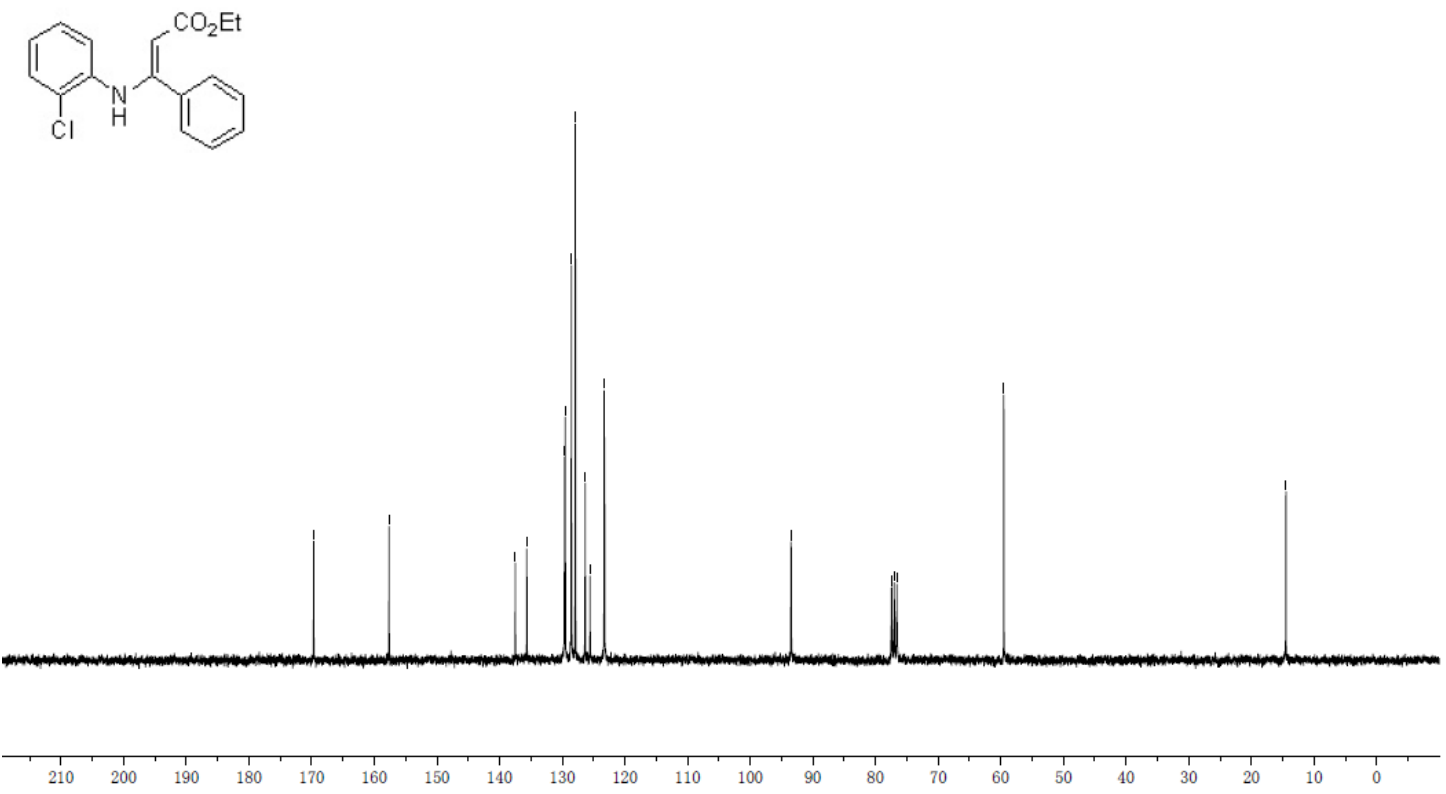

S32 
10

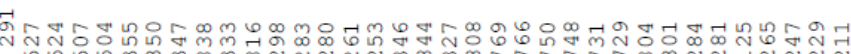

०.

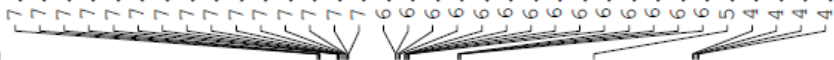

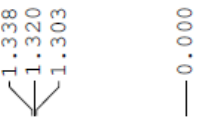

$\overbrace{\mathrm{Br}}^{\mathrm{Ph}}$

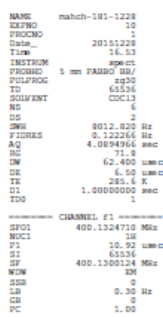
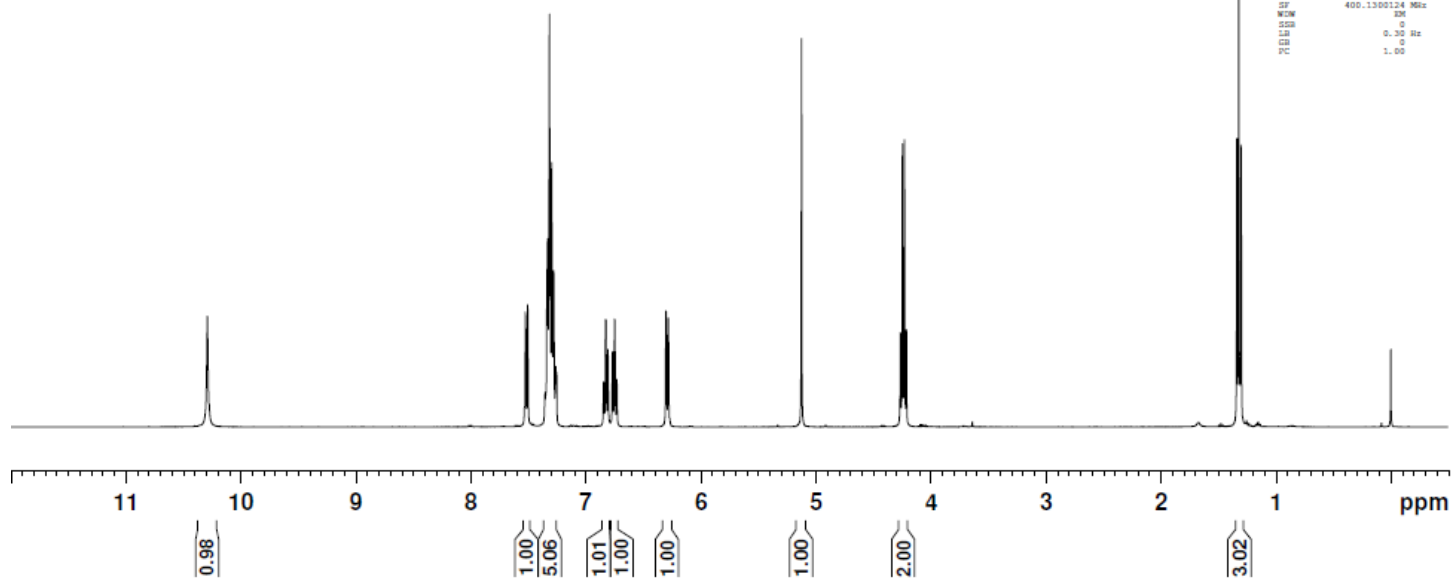

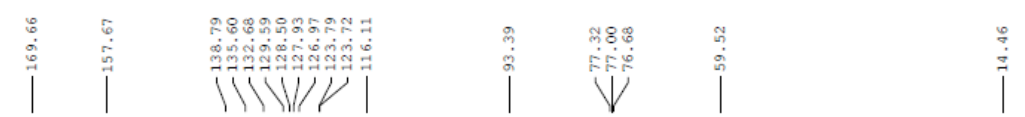

$\overbrace{\mathrm{Br}}^{\mathrm{Ph}}$

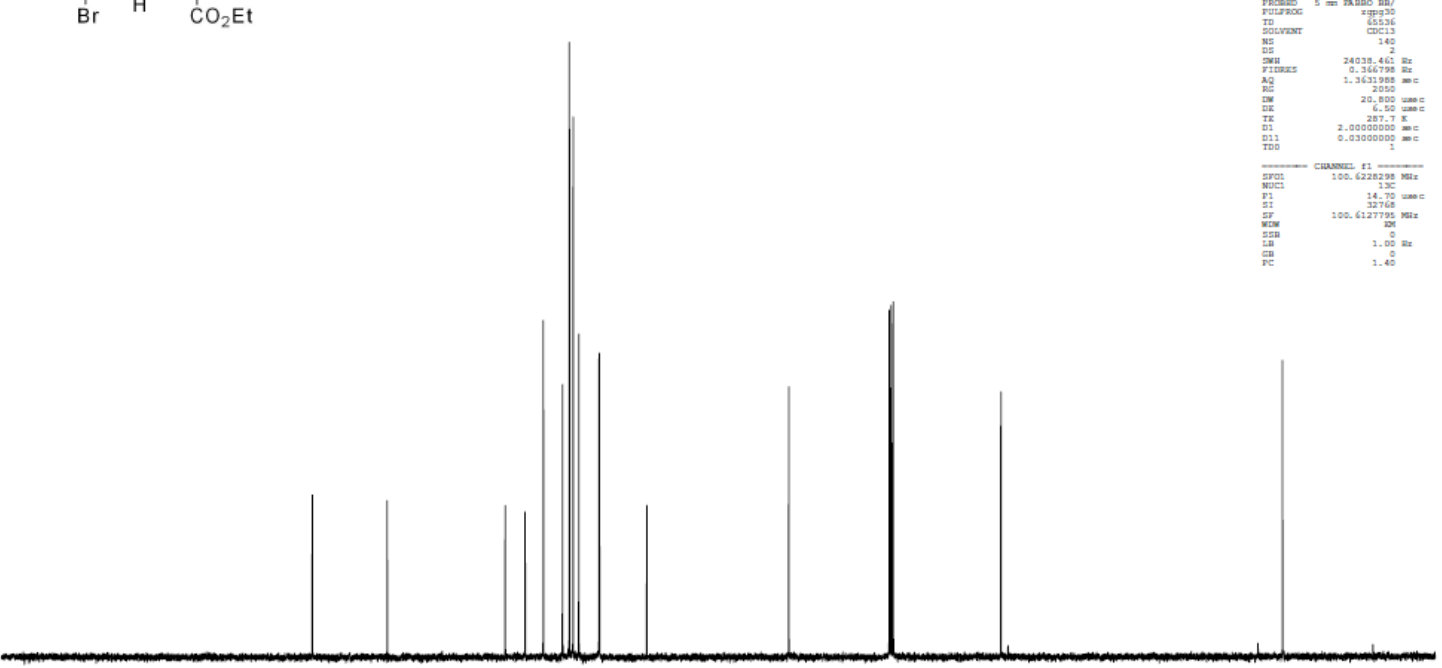

$\begin{array}{llllllllllllllllllllll}210 & 200 & 190 & 180 & 170 & 160 & 150 & 140 & 130 & 120 & 110 & 100 & 90 & 80 & 70 & 60 & 50 & 40 & 30 & 20 & 10 & \text { ppm }\end{array}$ 
$1 p$

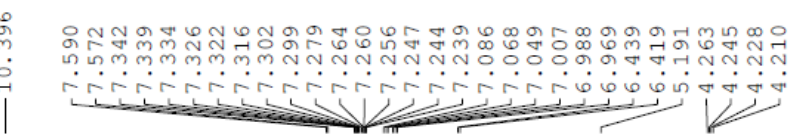

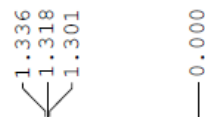

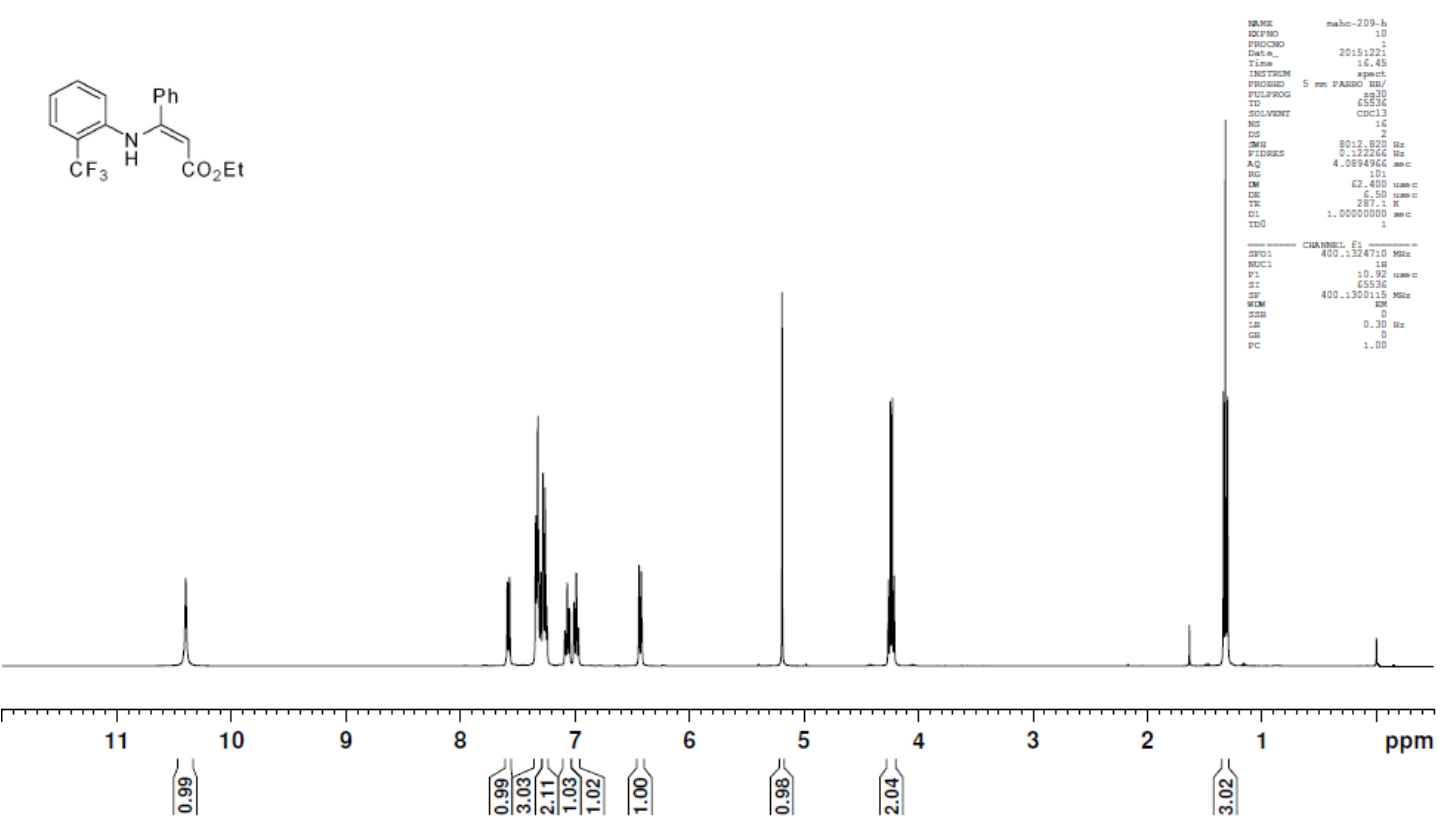

| |
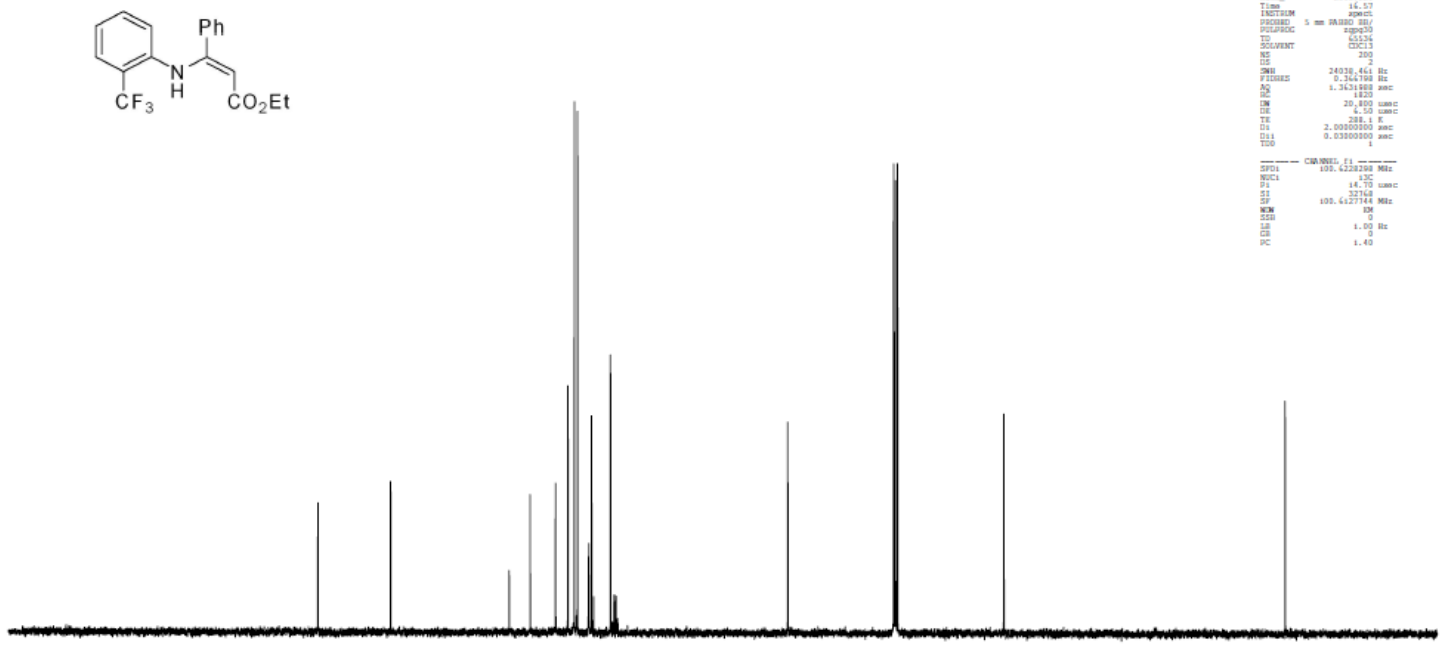

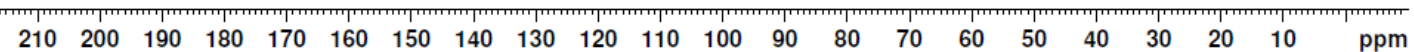


$1 \mathbf{q}$

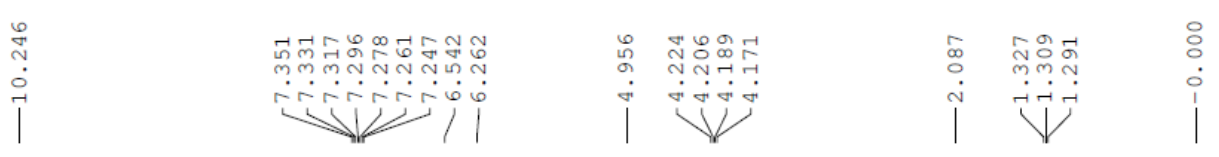<smiles>CCOCC(C)Nc1ccc(C)cc1</smiles>

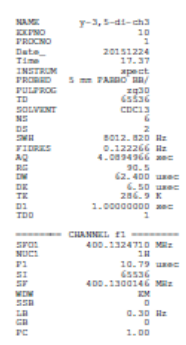

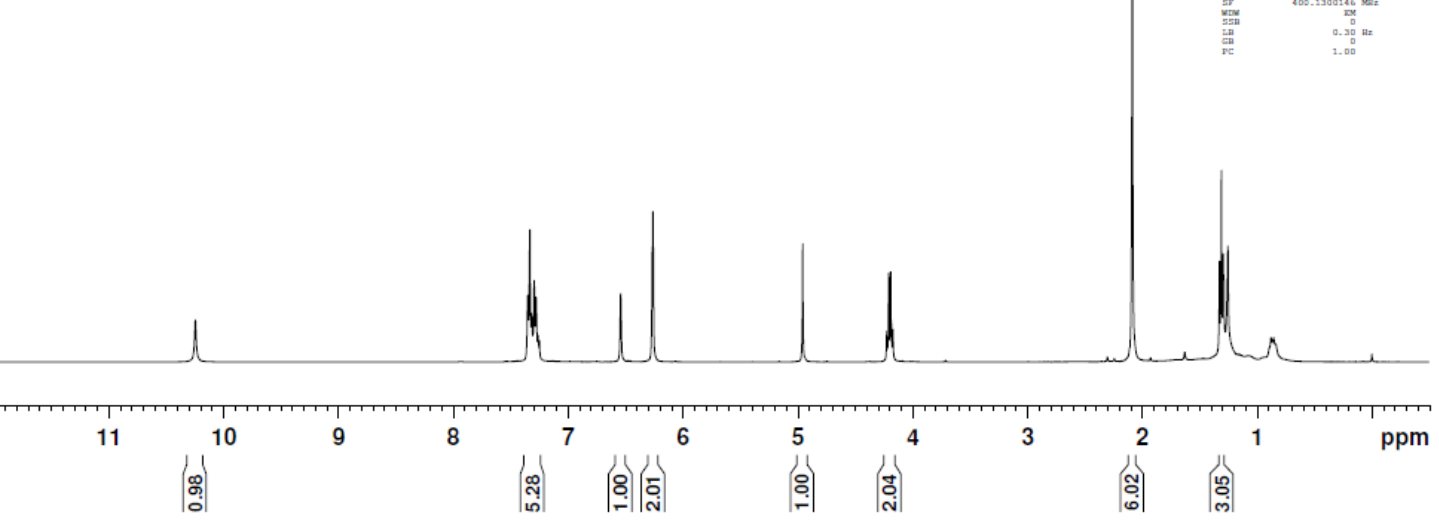

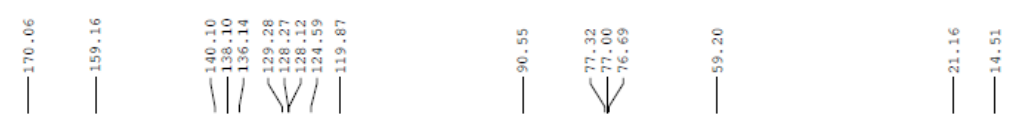<smiles>CCOCC(C)Nc1cc(C)cc(C)c1</smiles>

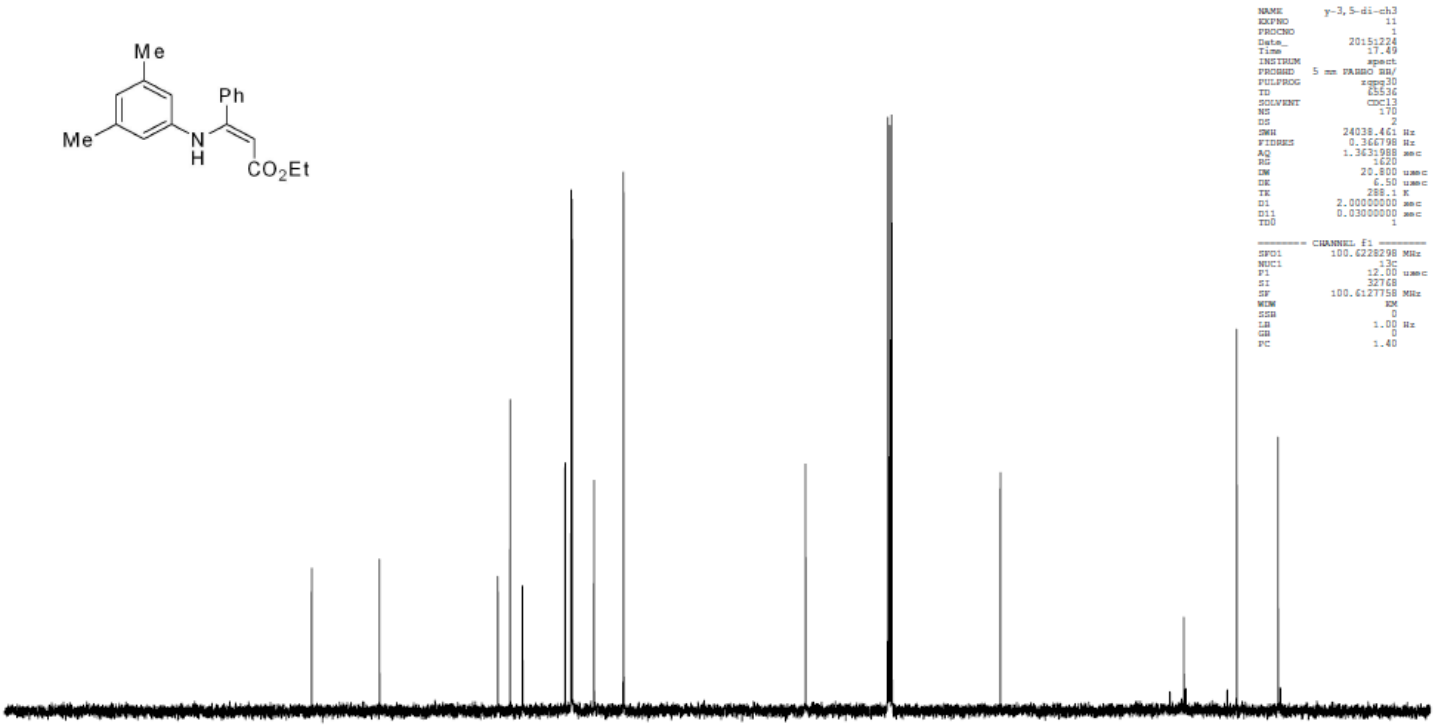

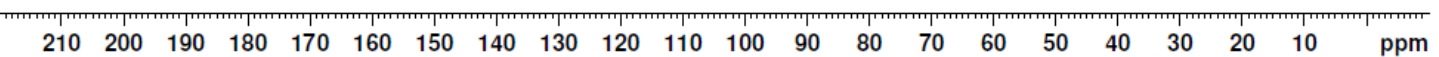


1r

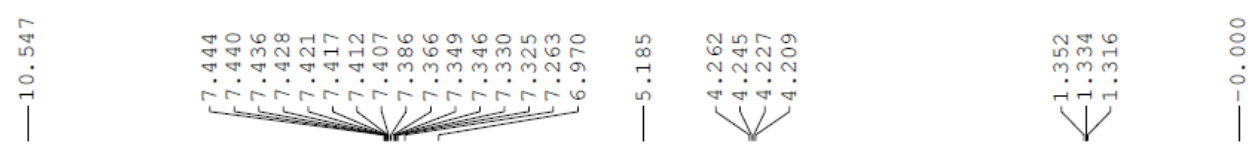

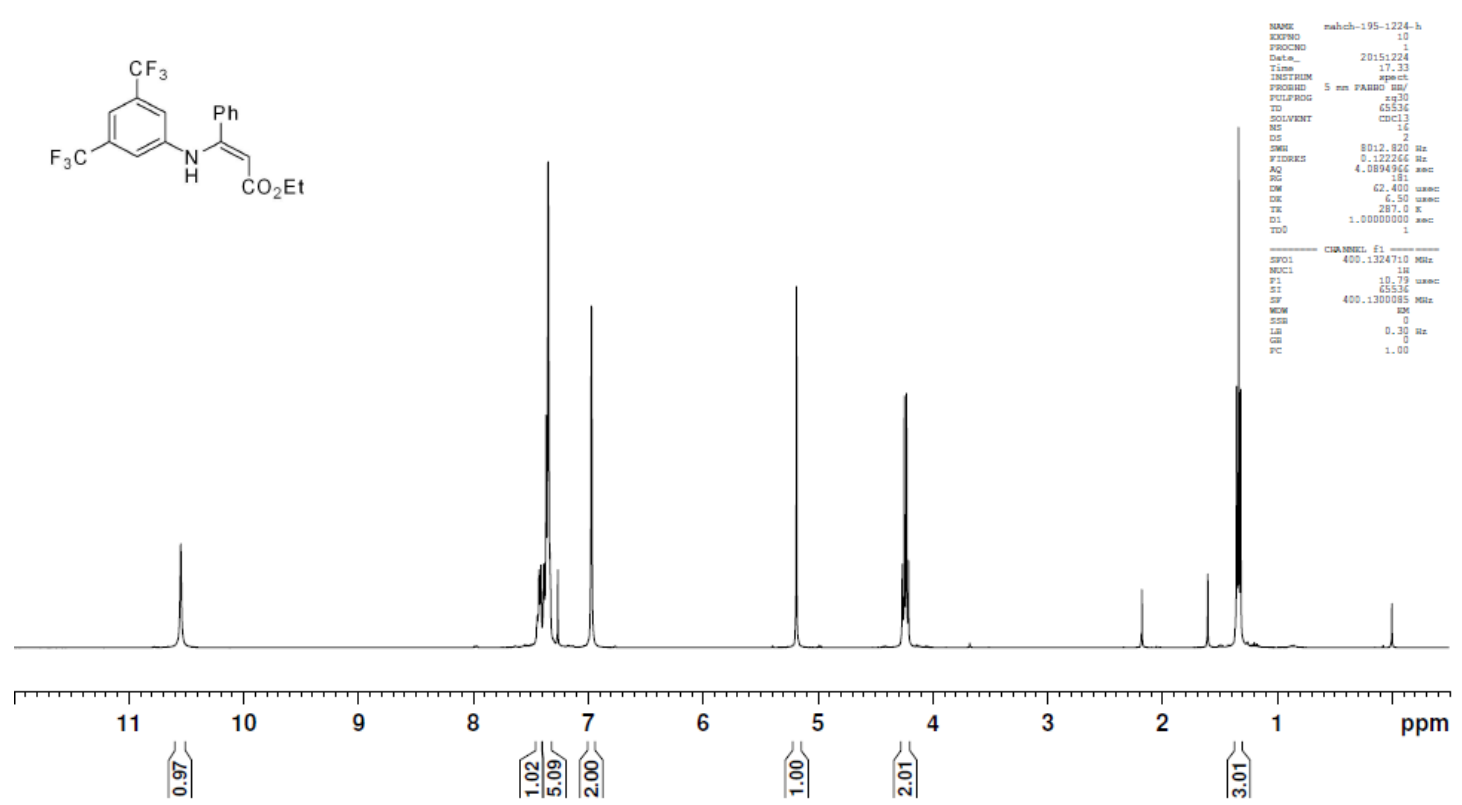

|
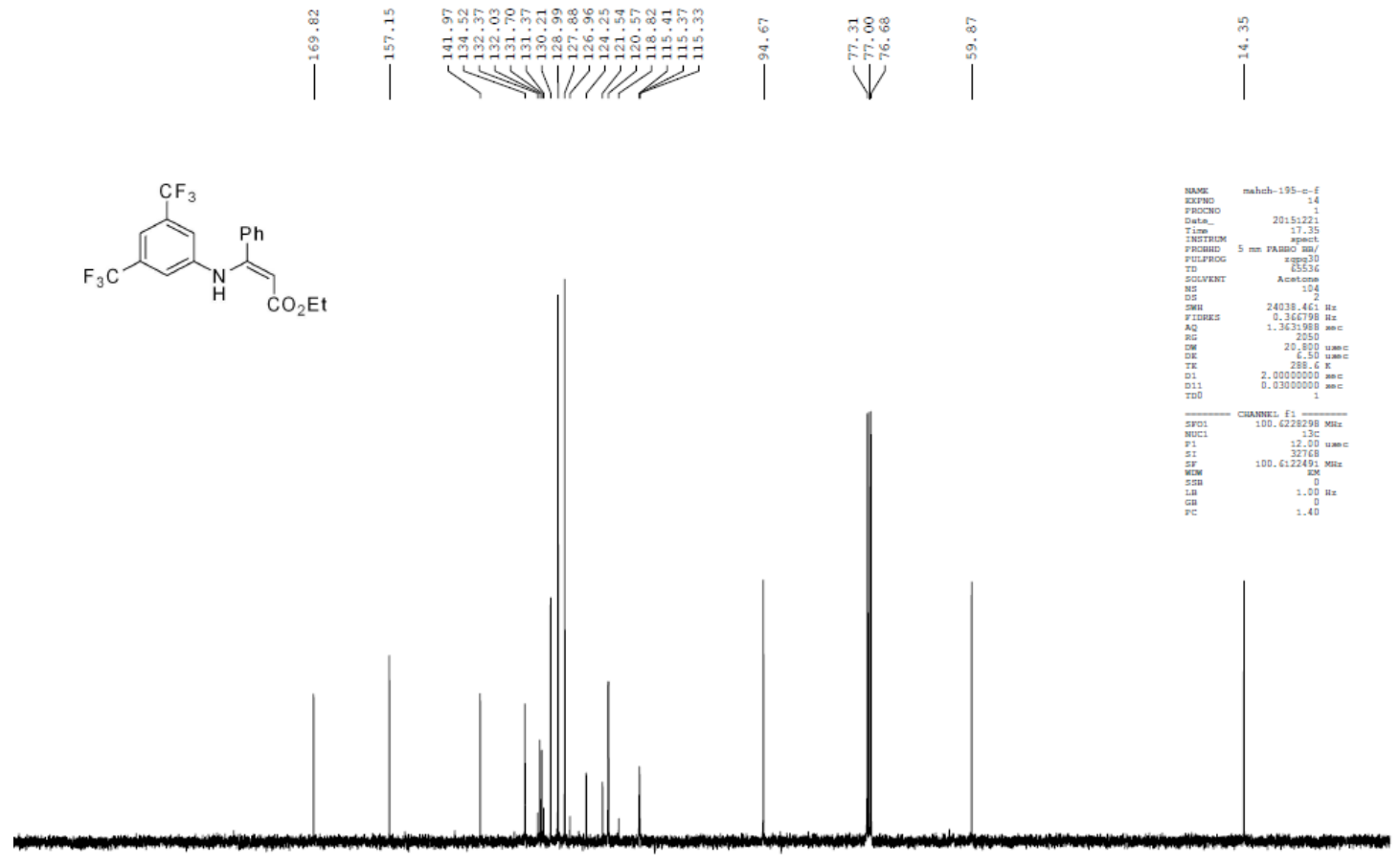

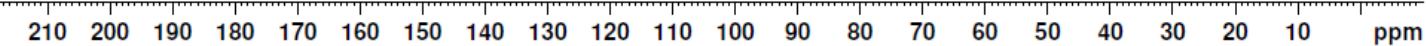


1s

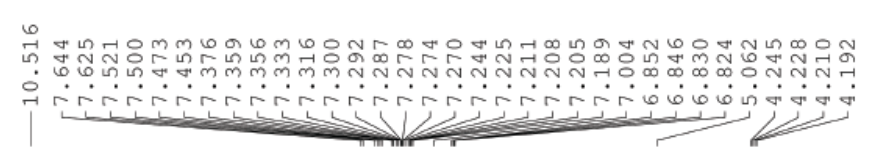

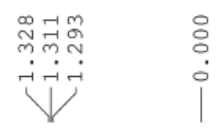
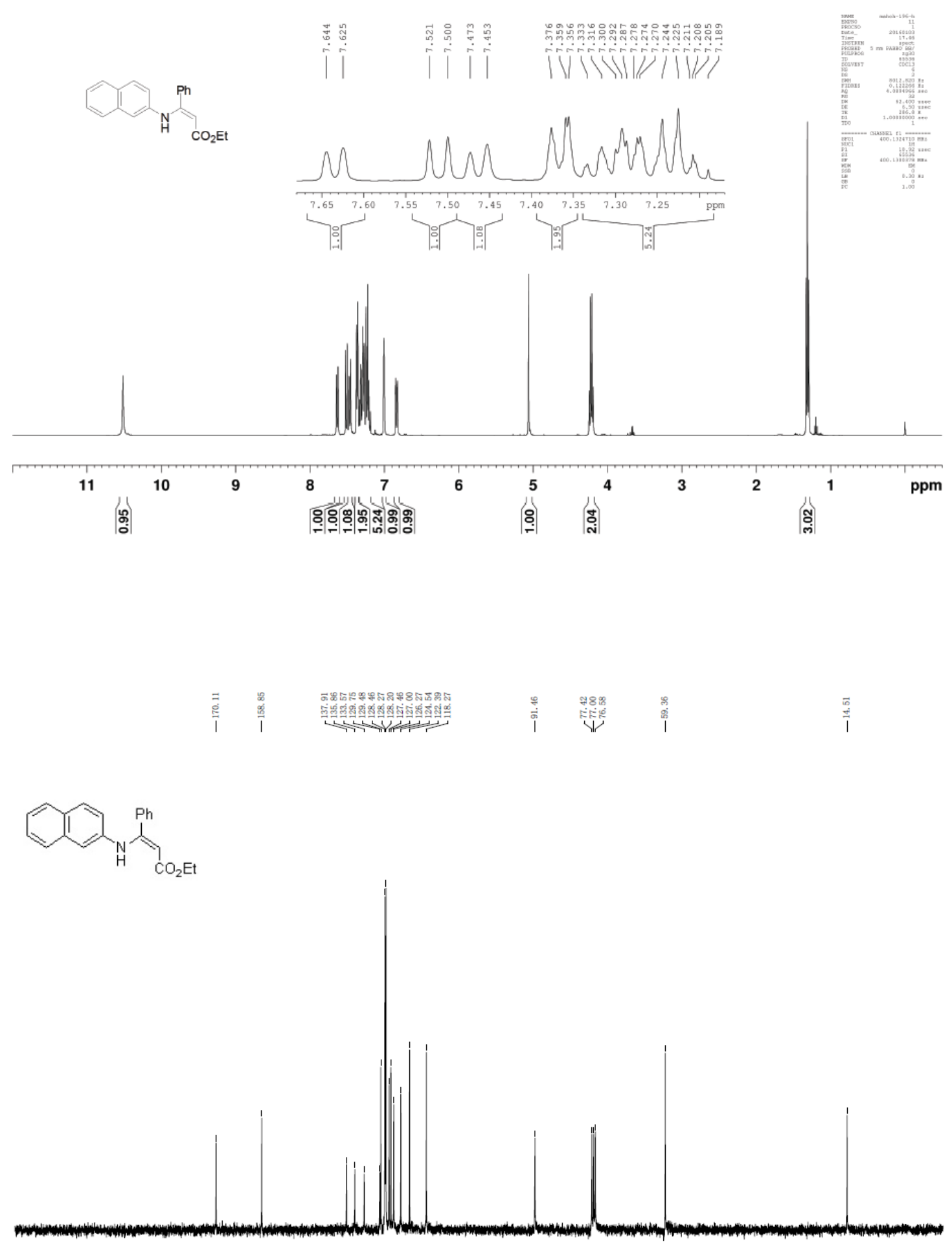
1t

$\underbrace{1}$
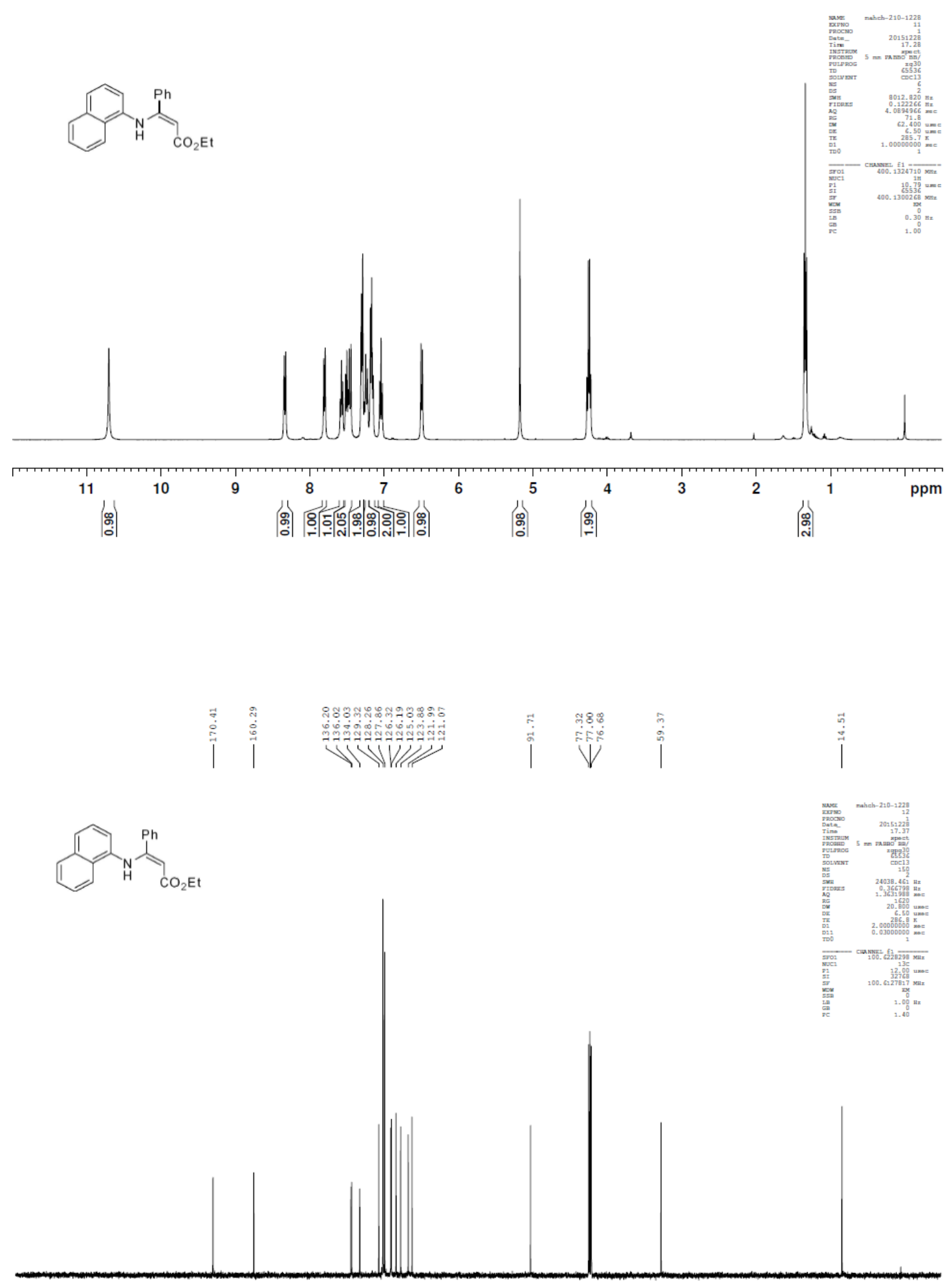

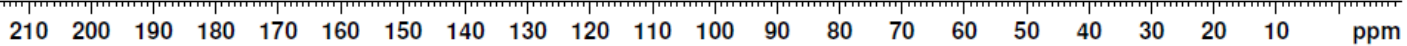




\section{1u}

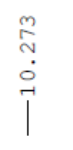

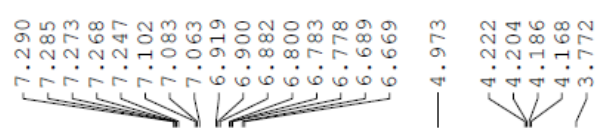

Vंग
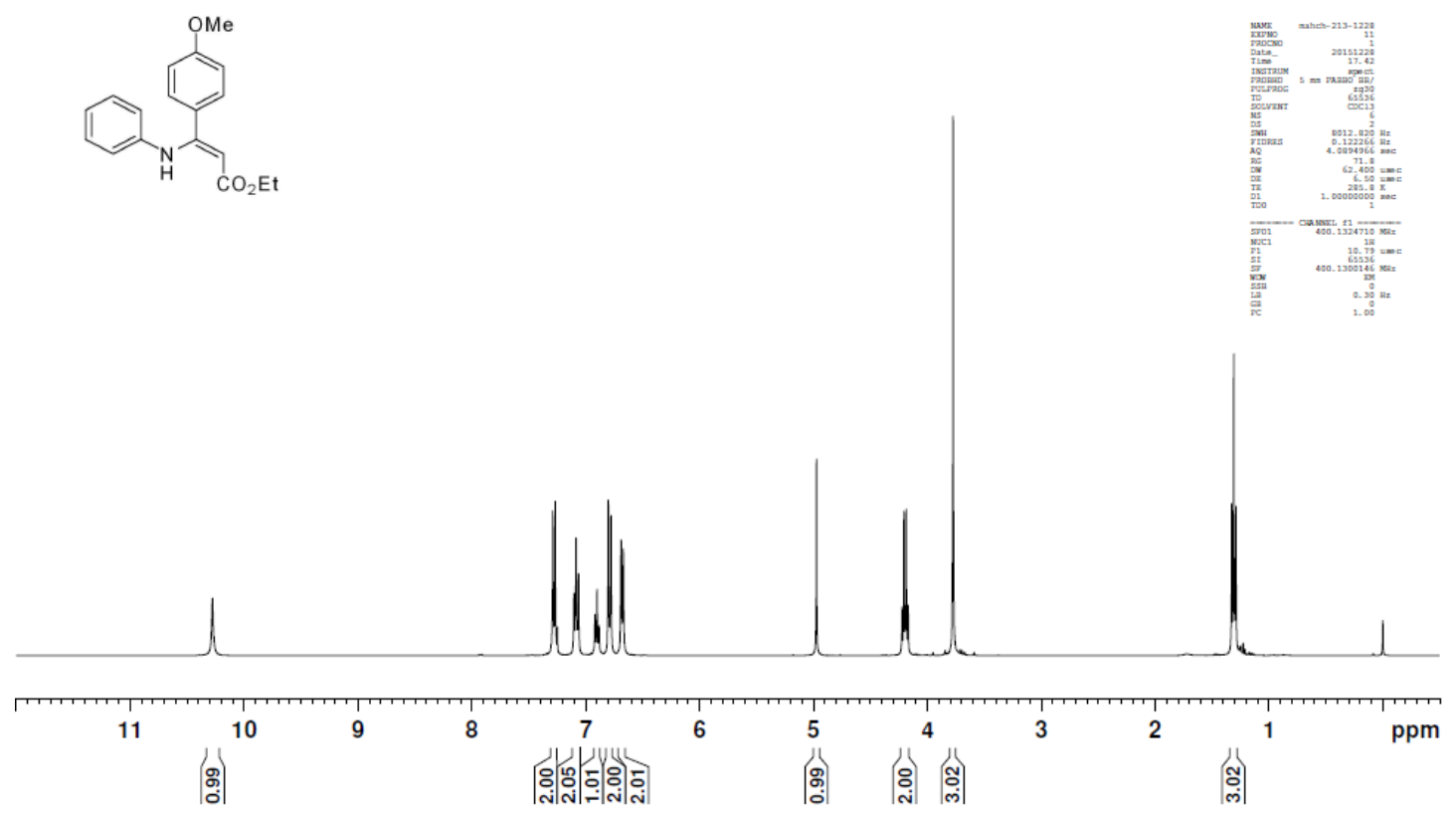

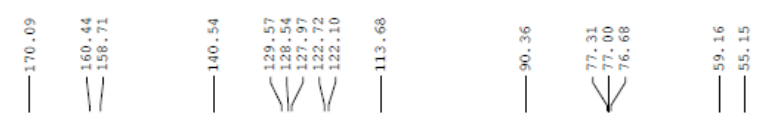
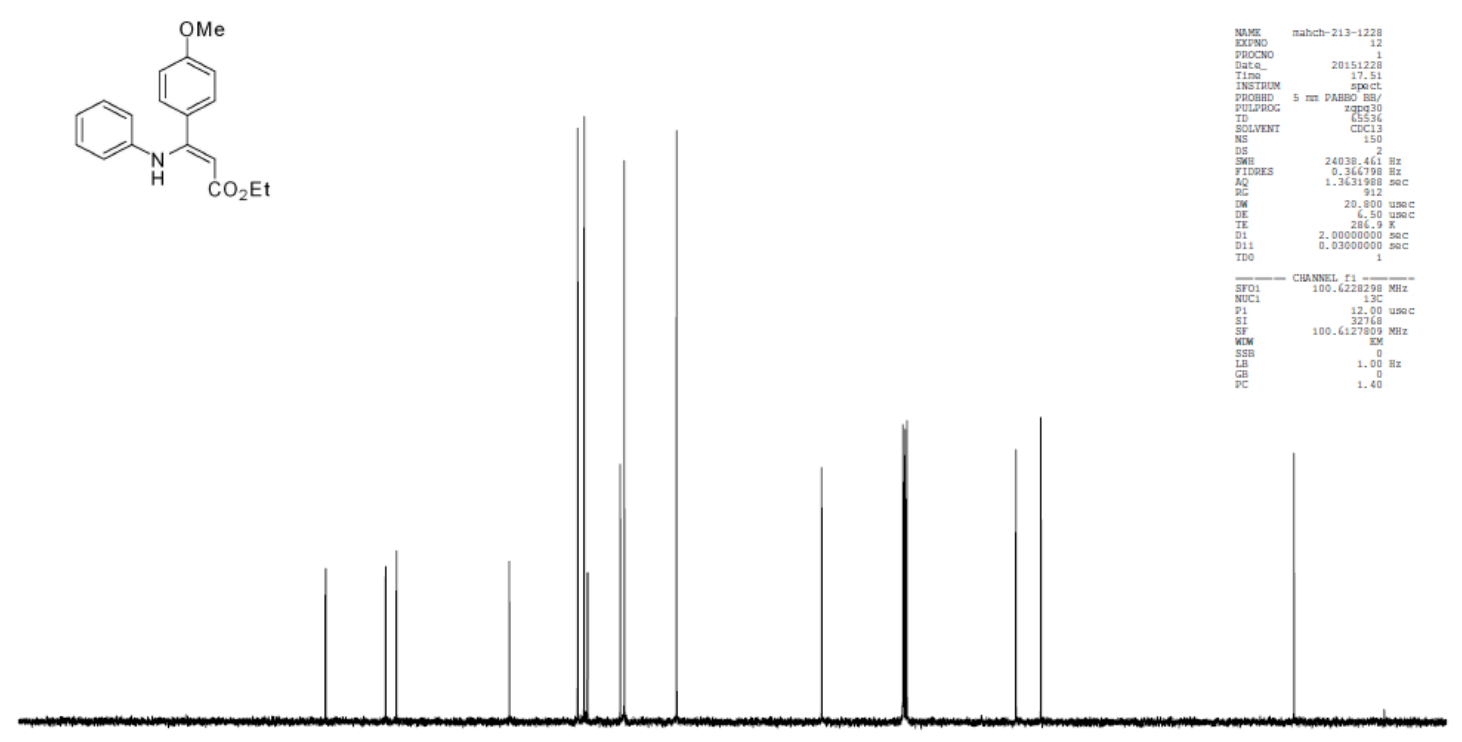

$\begin{array}{lllllllllllllllllllll}210 & 200 & 190 & 180 & 170 & 160 & 150 & 140 & 130 & 120 & 110 & 100 & 90 & 80 & 70 & 60 & 50 & 40 & 30 & 20 & 10\end{array}$ 
1v

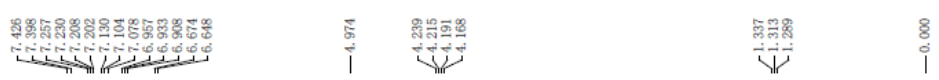<smiles>CCOC(=O)/C=C(/Nc1ccccc1)c1ccc(Br)cc1</smiles>

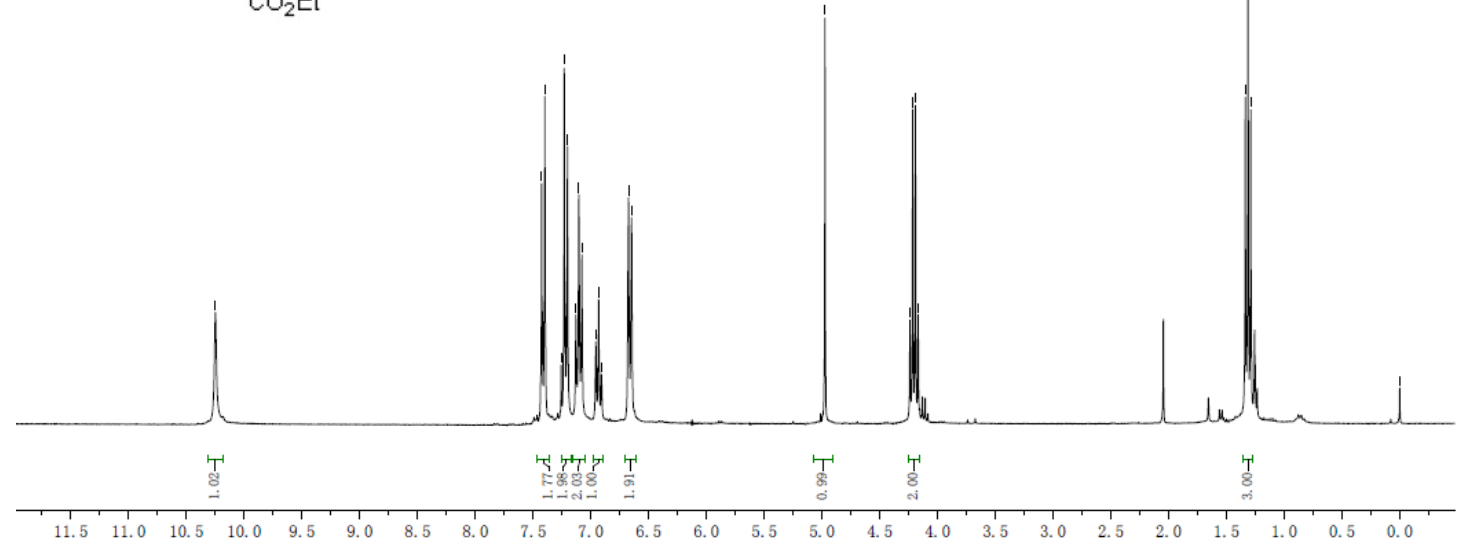

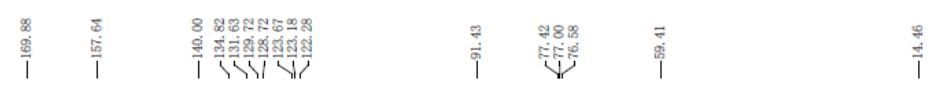<smiles>CCOC(=O)/C=C(/Nc1ccccc1)c1ccc(Br)cc1</smiles>

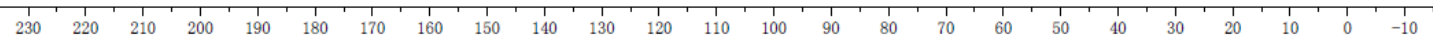


$\mathbf{1 w}$

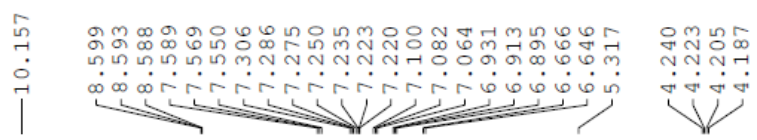

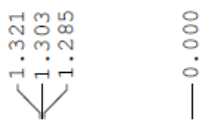<smiles></smiles>
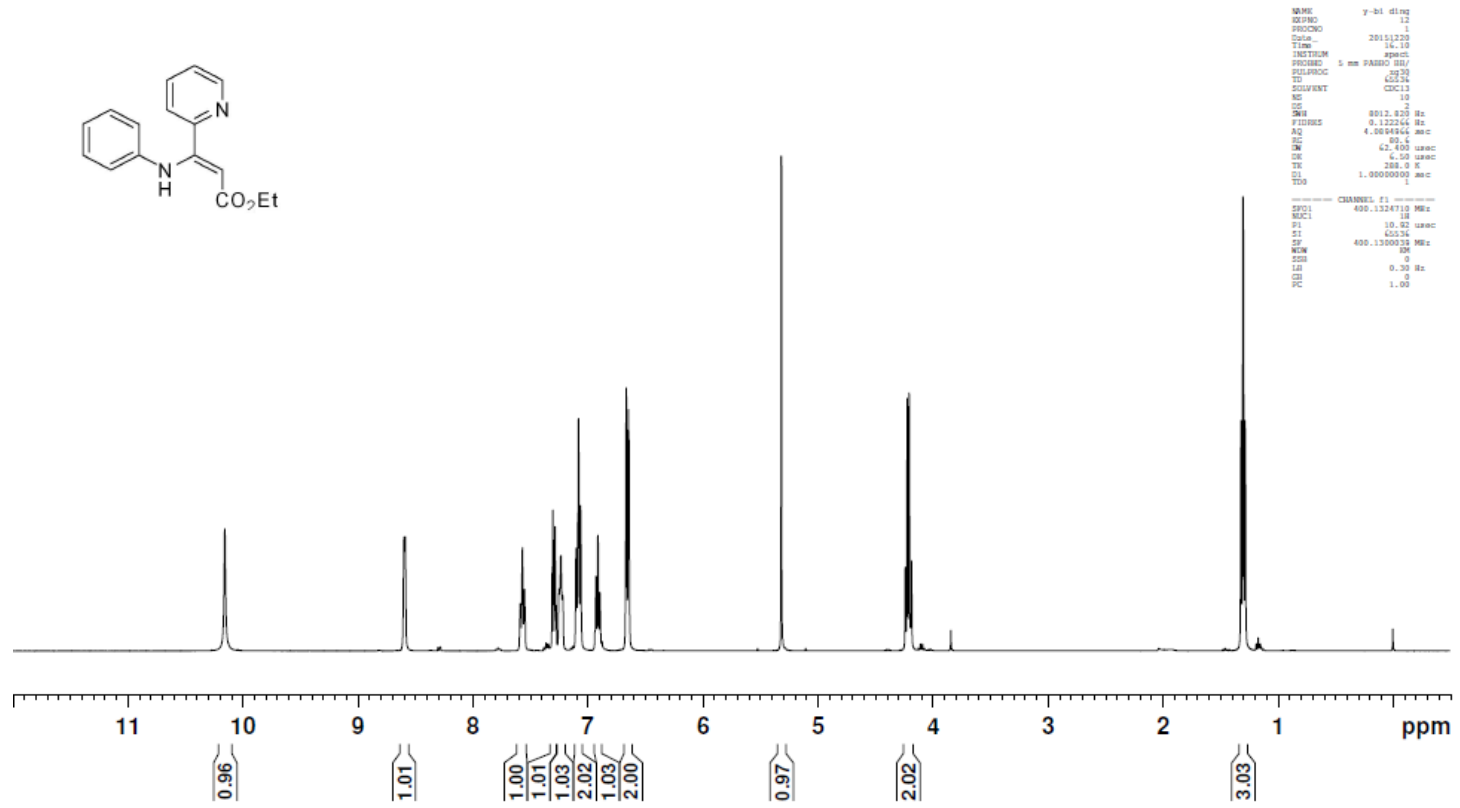

|
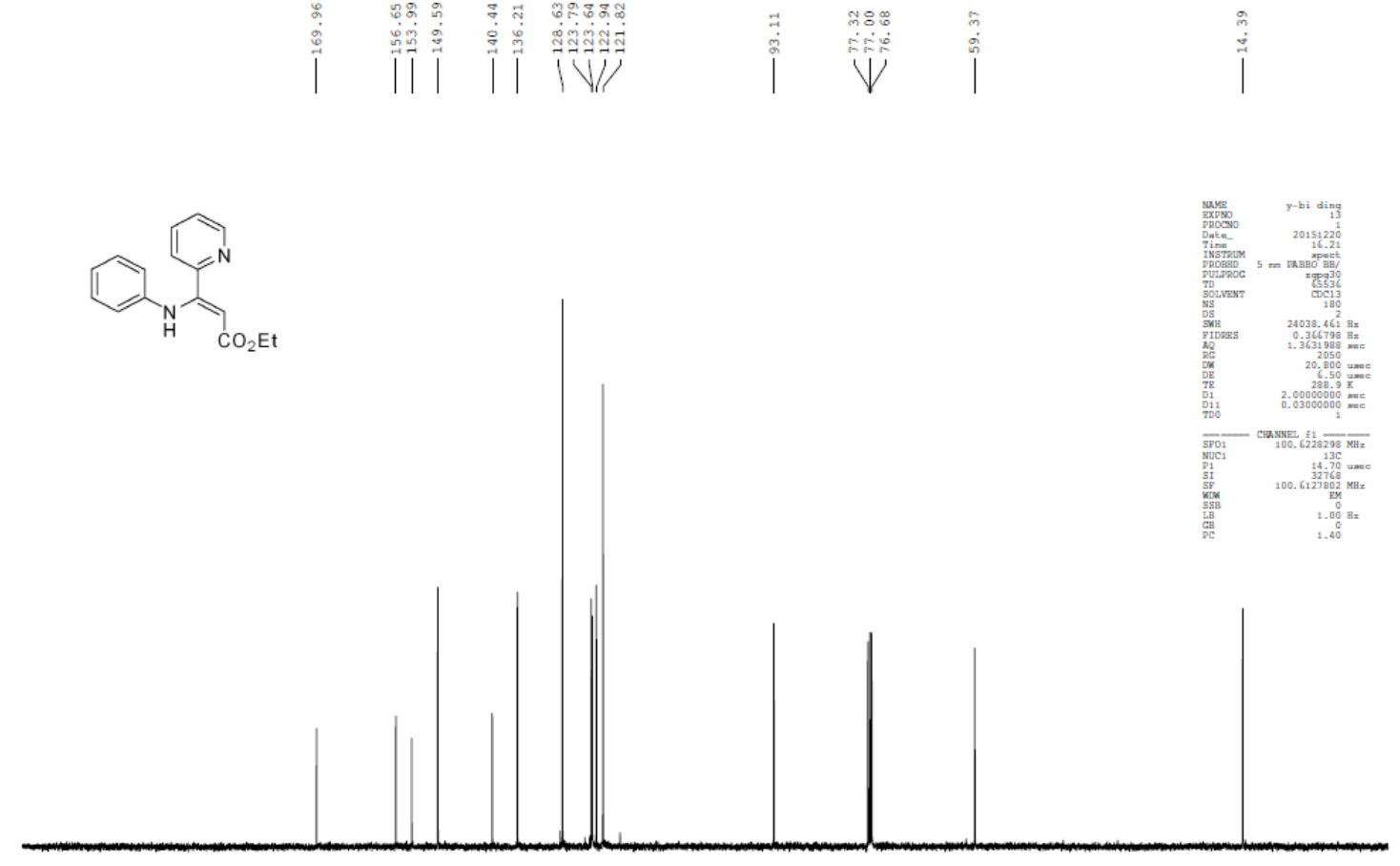

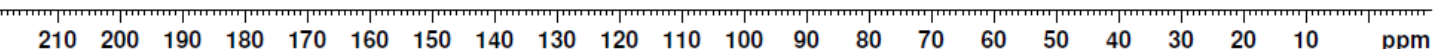


1x
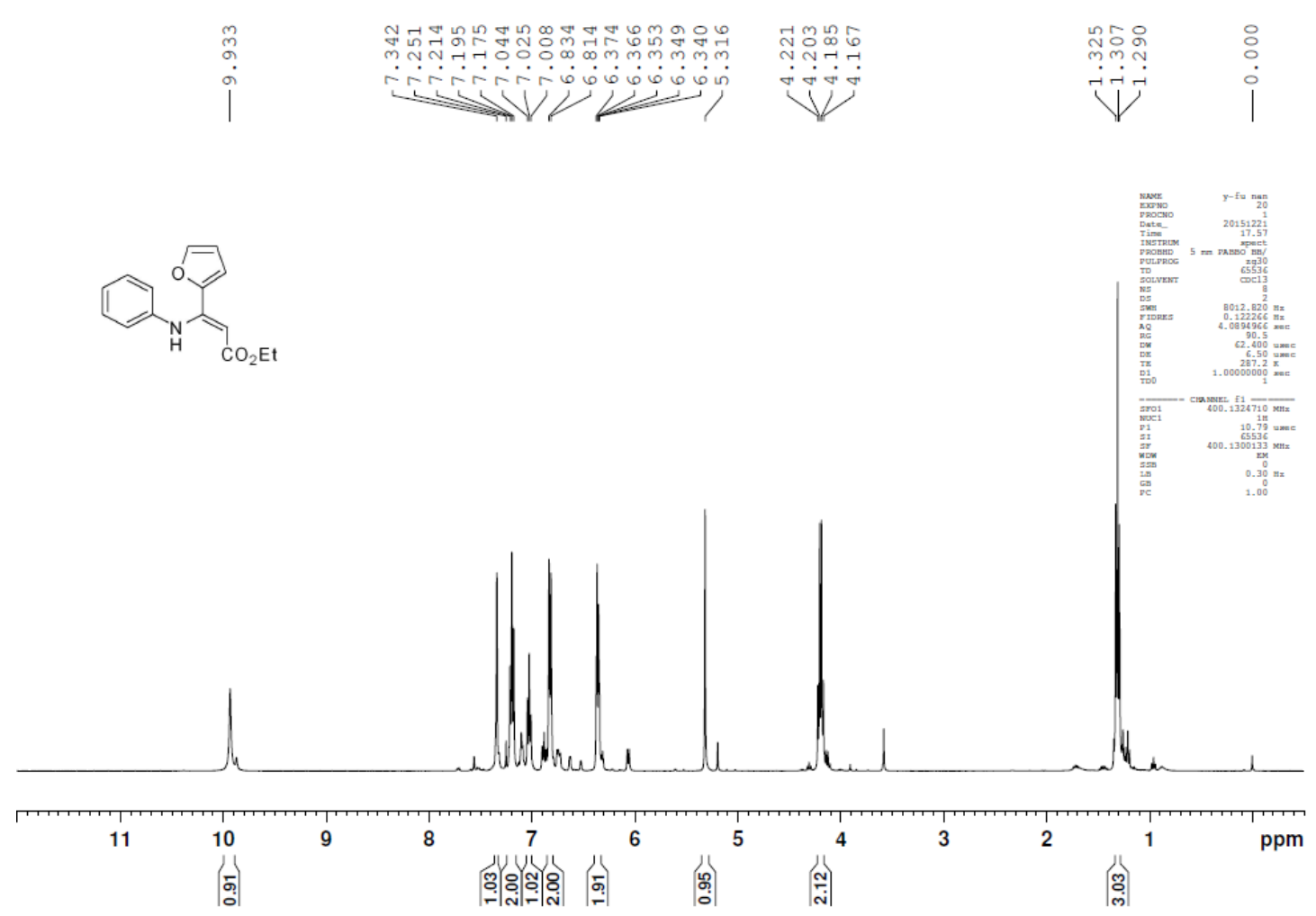

|<smiles>CCOCC(C=C(Nc1ccccc1)c1ccco1)OCC</smiles>

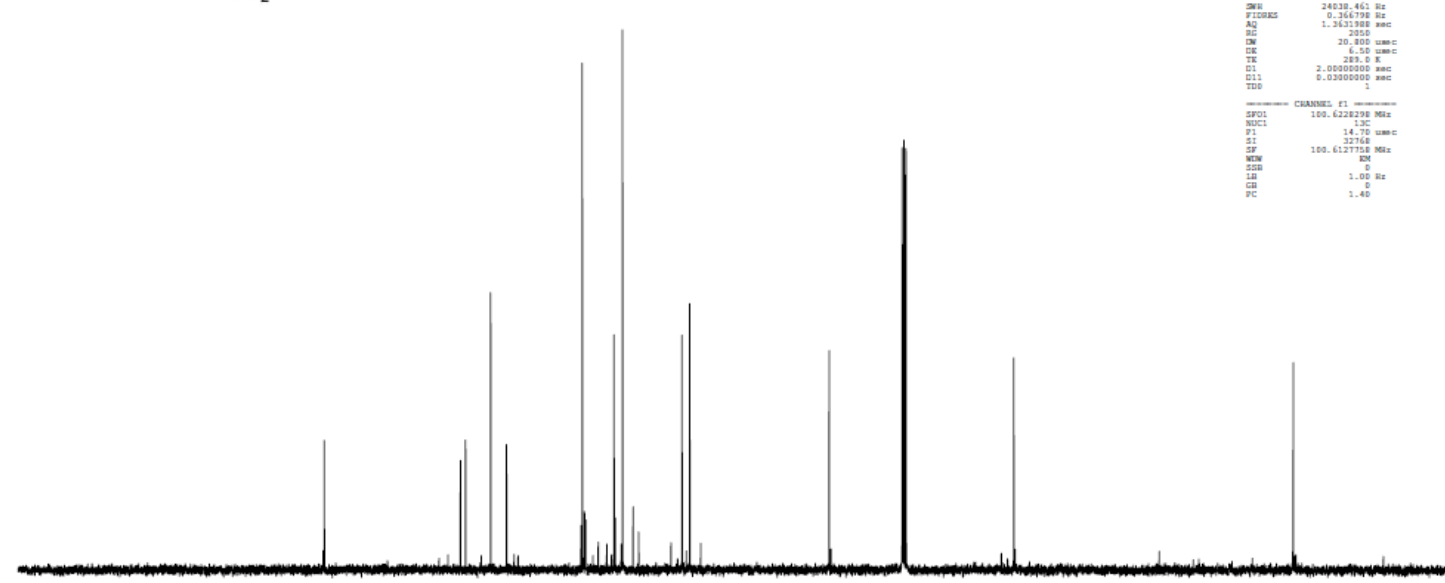

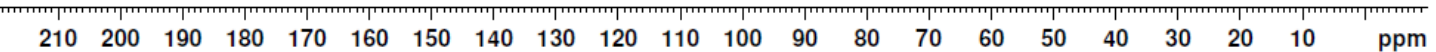


3
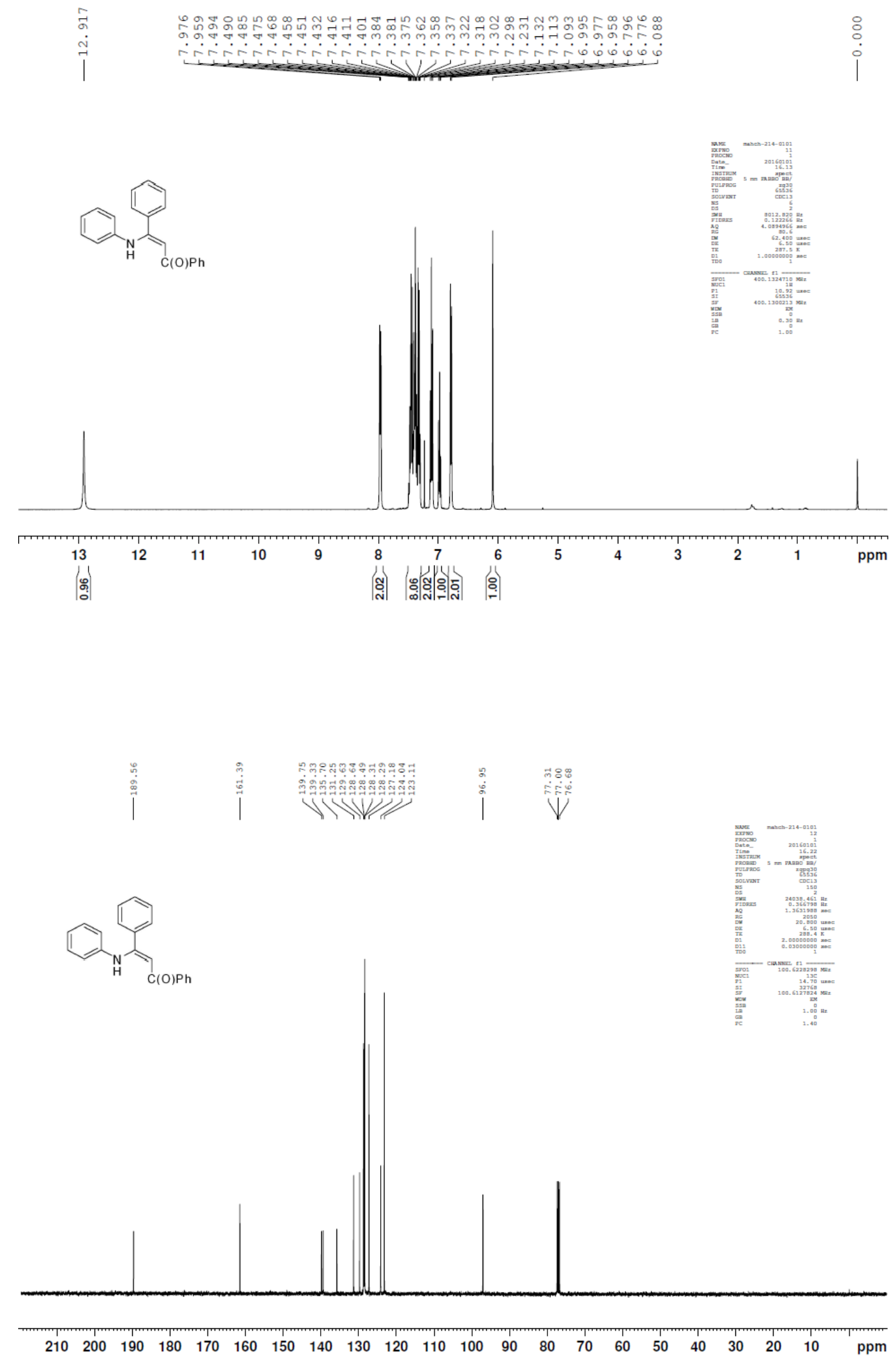
4
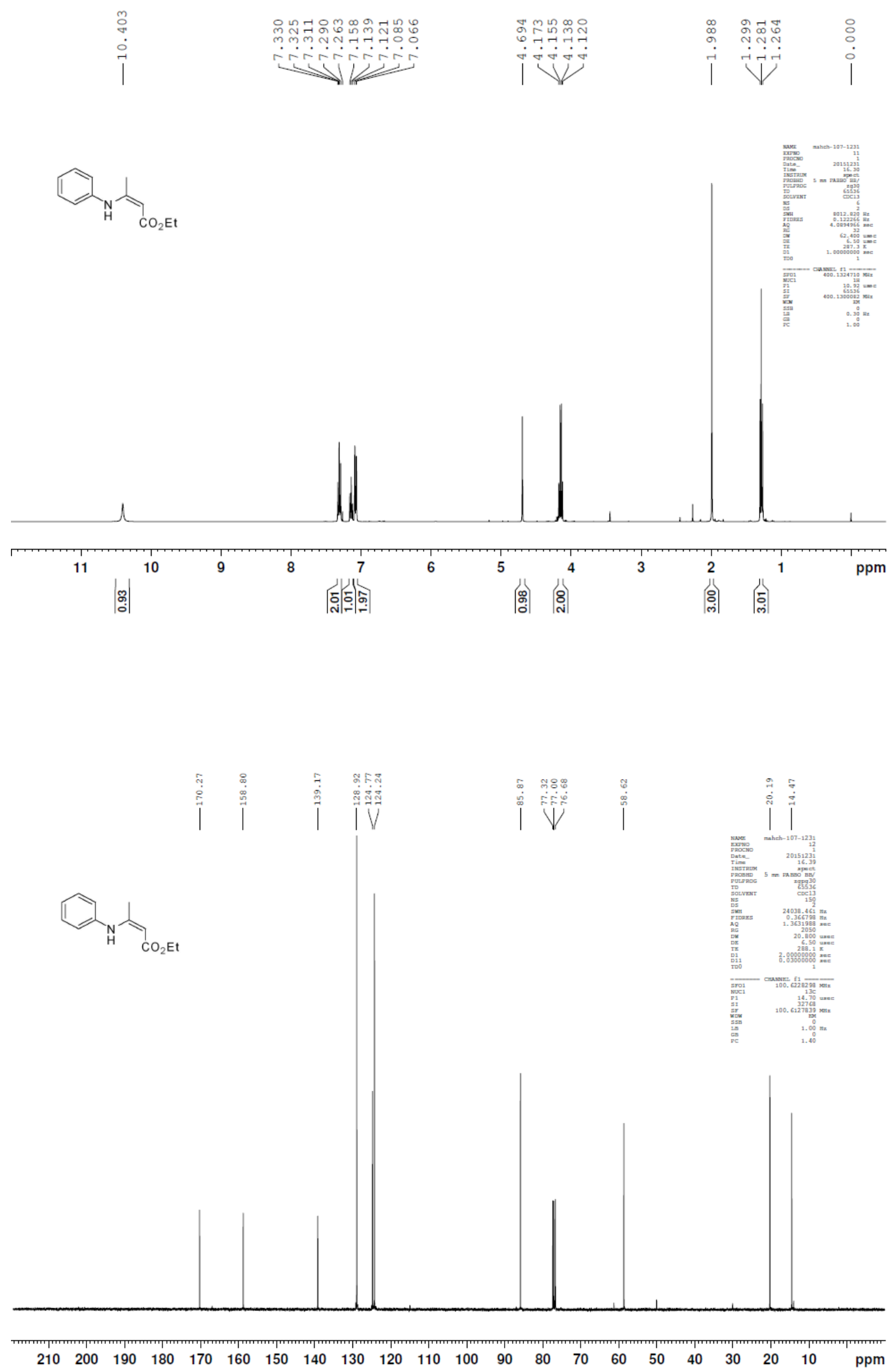
6

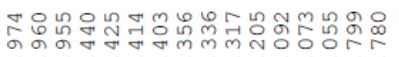

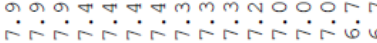

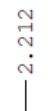

$\circ$
$\vdots$
$\dot{0}$<smiles>CC(Nc1ccccc1)=C(C)c1ccccc1</smiles>
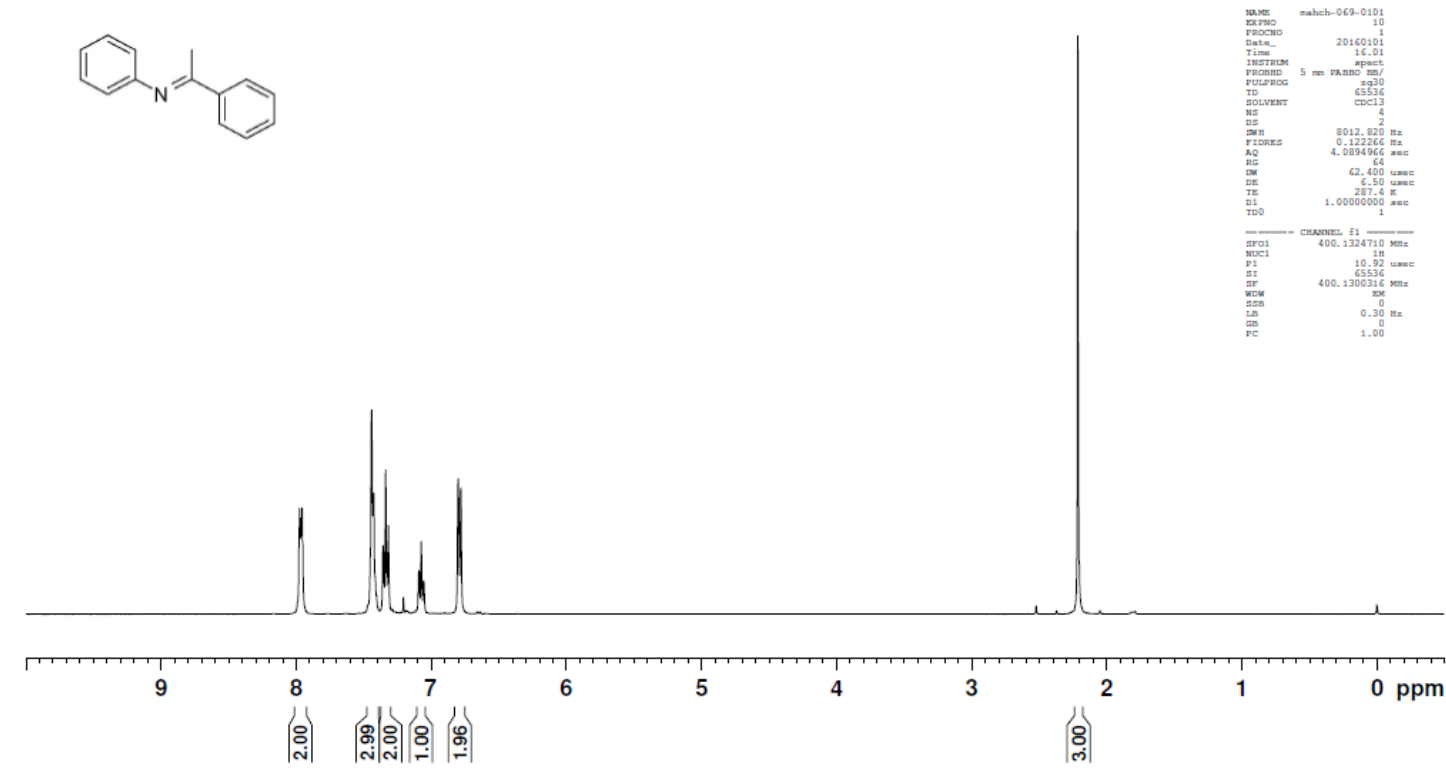

|

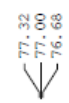

$\stackrel{\vec{m}}{\overrightarrow{\vec{n}}}$

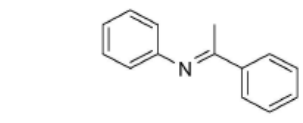

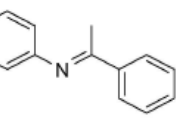

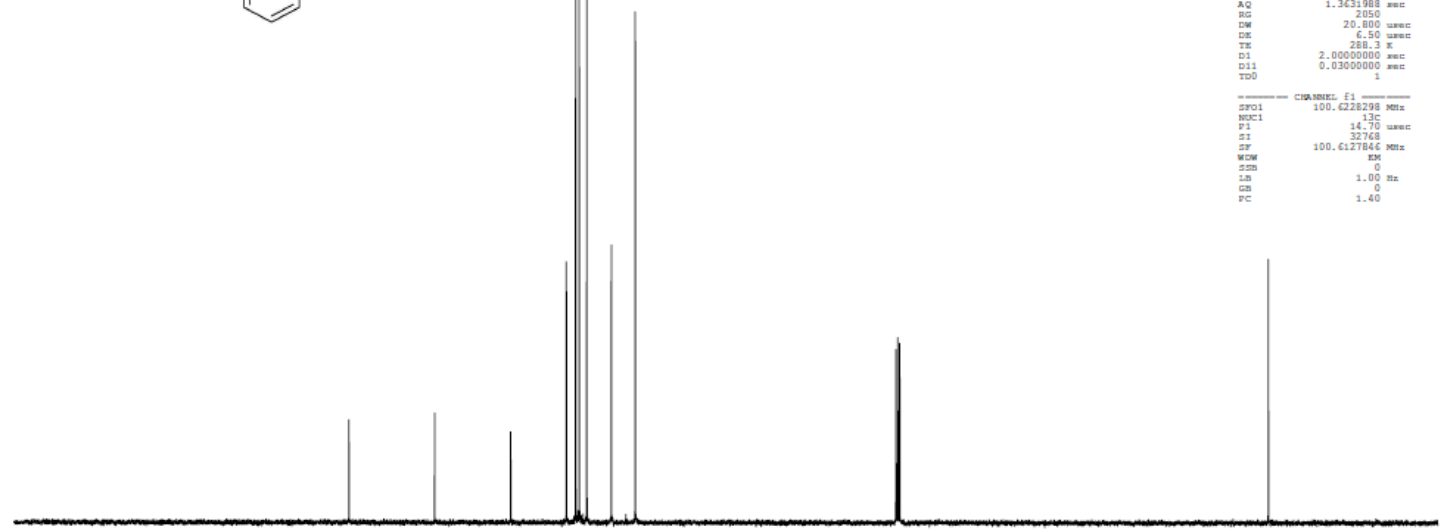

$\begin{array}{lllllllllllllllllllll}210 & 200 & 190 & 180 & 170 & 160 & 150 & 140 & 130 & 120 & 110 & 100 & 90 & 80 & 70 & 60 & 50 & 40 & 30 & 20 & 10\end{array}$ 


\section{Copies of ${ }^{1} \mathrm{H}$ NMR and ${ }^{13} \mathrm{C}$ NMR spectra of the products}

2a
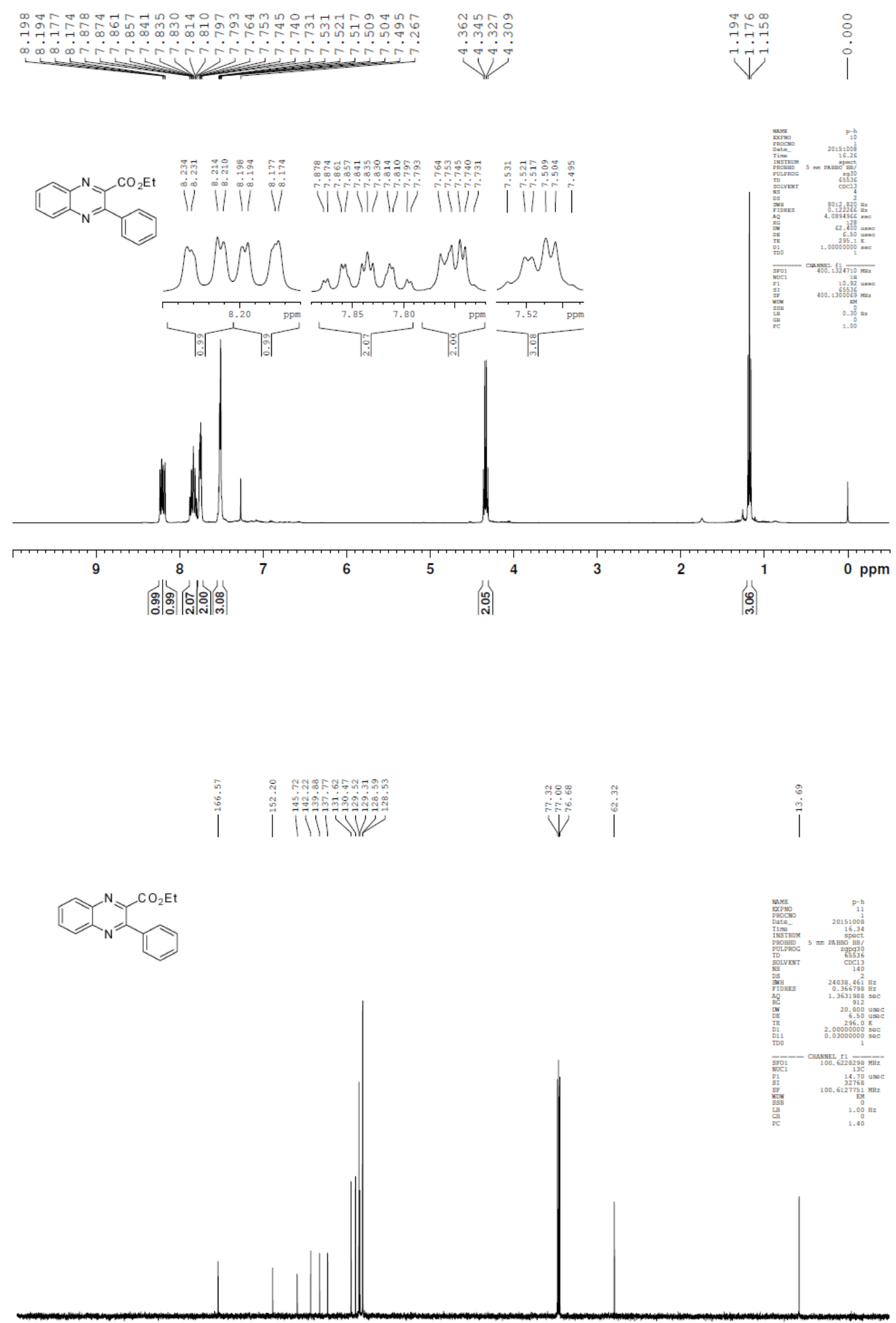

$\begin{array}{lllllllllllllllllllll}210 & 200 & 190 & 180 & 170 & 160 & 150 & 140 & 130 & 120 & 110 & 100 & 90 & 80 & 70 & 60 & 50 & 40 & 30 & 20 & 10\end{array}$ 
2b

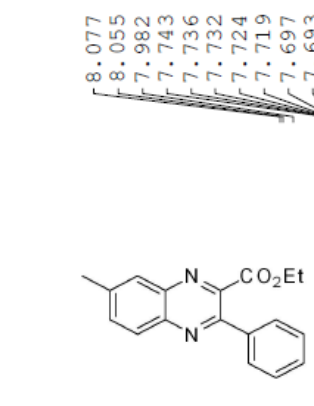

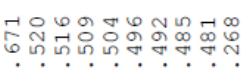

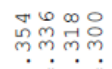

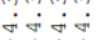

i
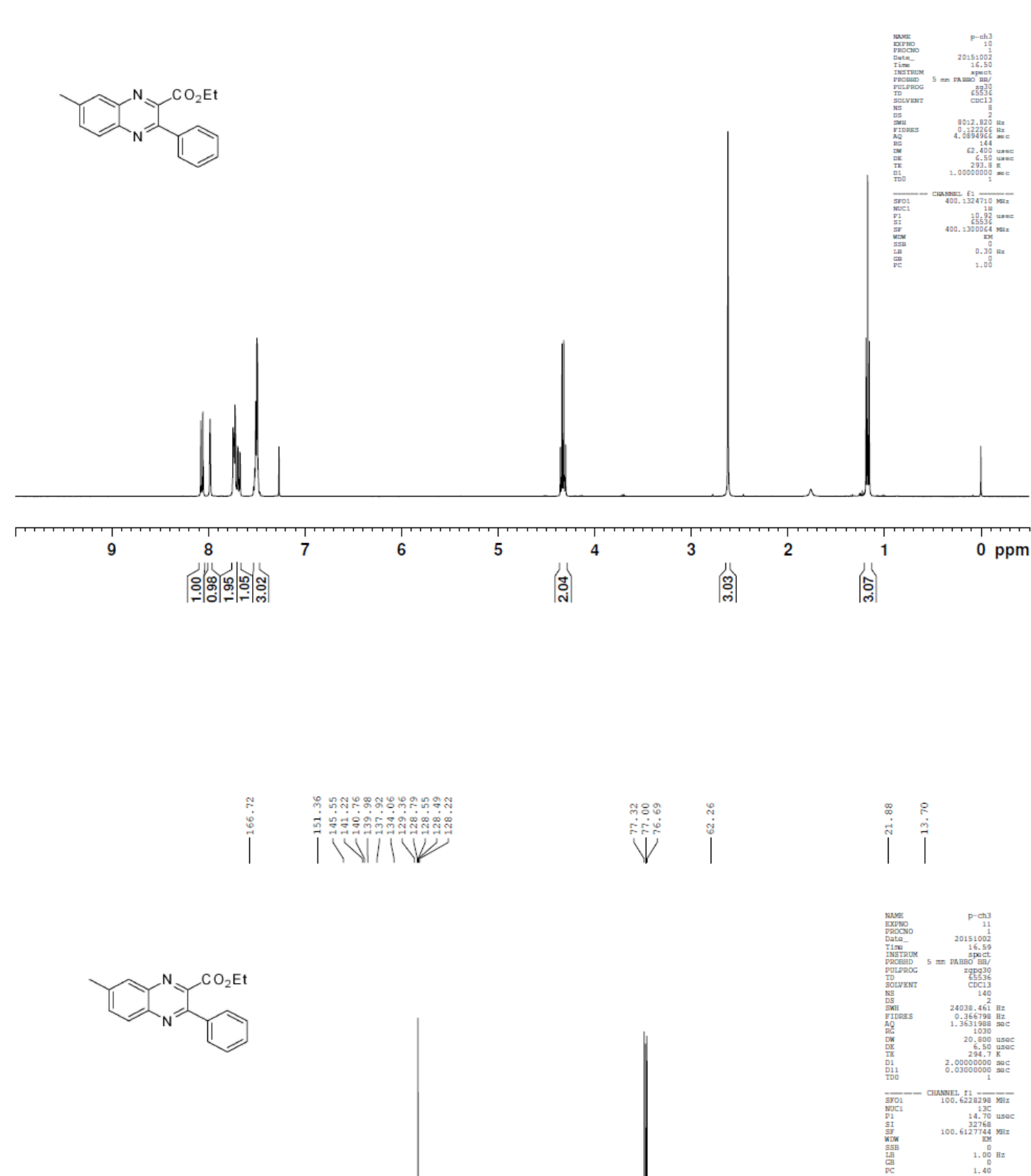

$\begin{array}{llllllllllllllllllllll}210 & 200 & 190 & 180 & 170 & 160 & 150 & 140 & 130 & 120 & 110 & 100 & 90 & 80 & 70 & 60 & 50 & 40 & 30 & 20 & 10 & \mathrm{ppm}\end{array}$ 


\section{2c}

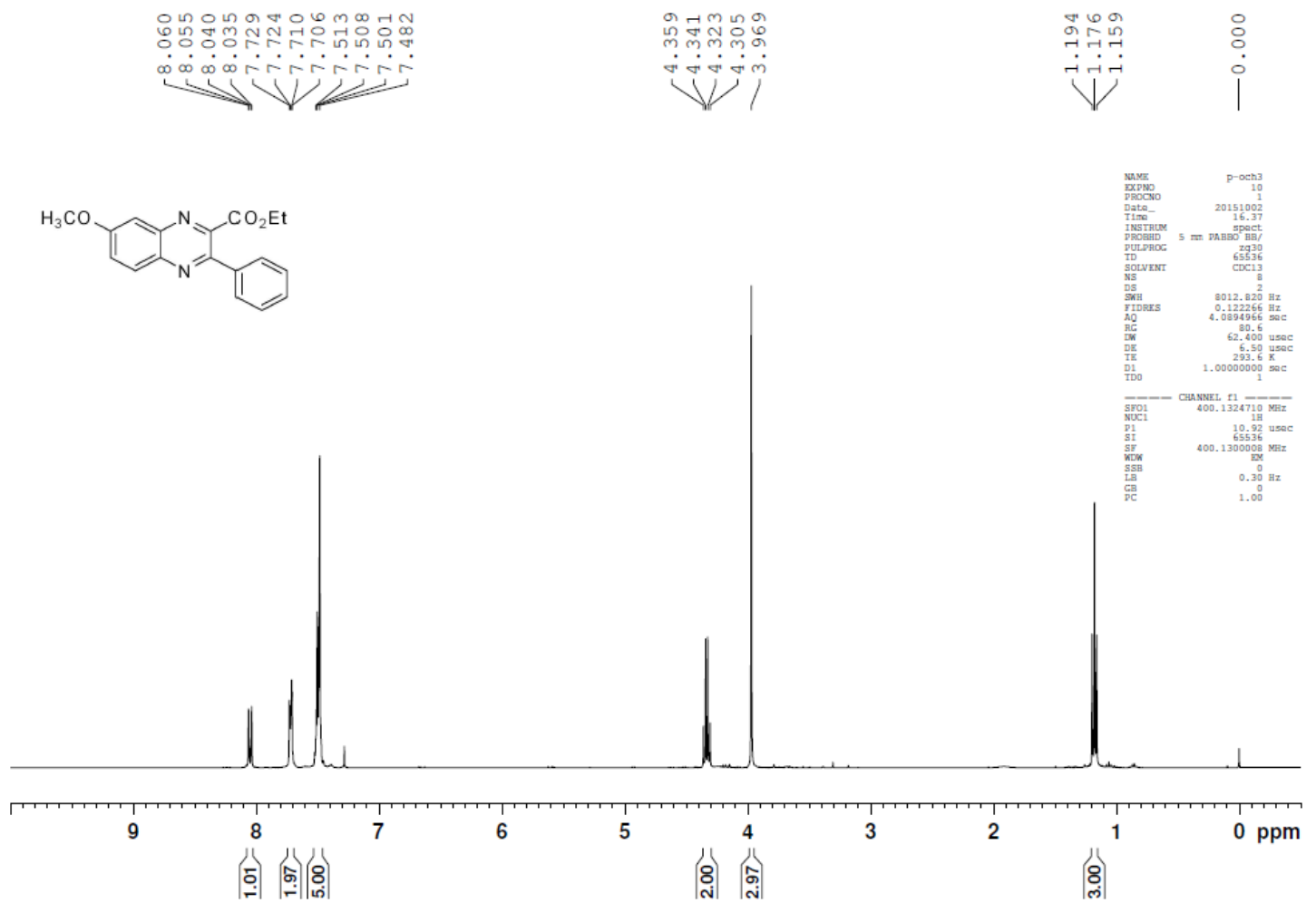

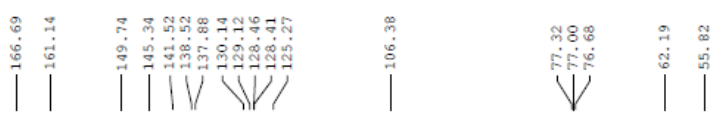
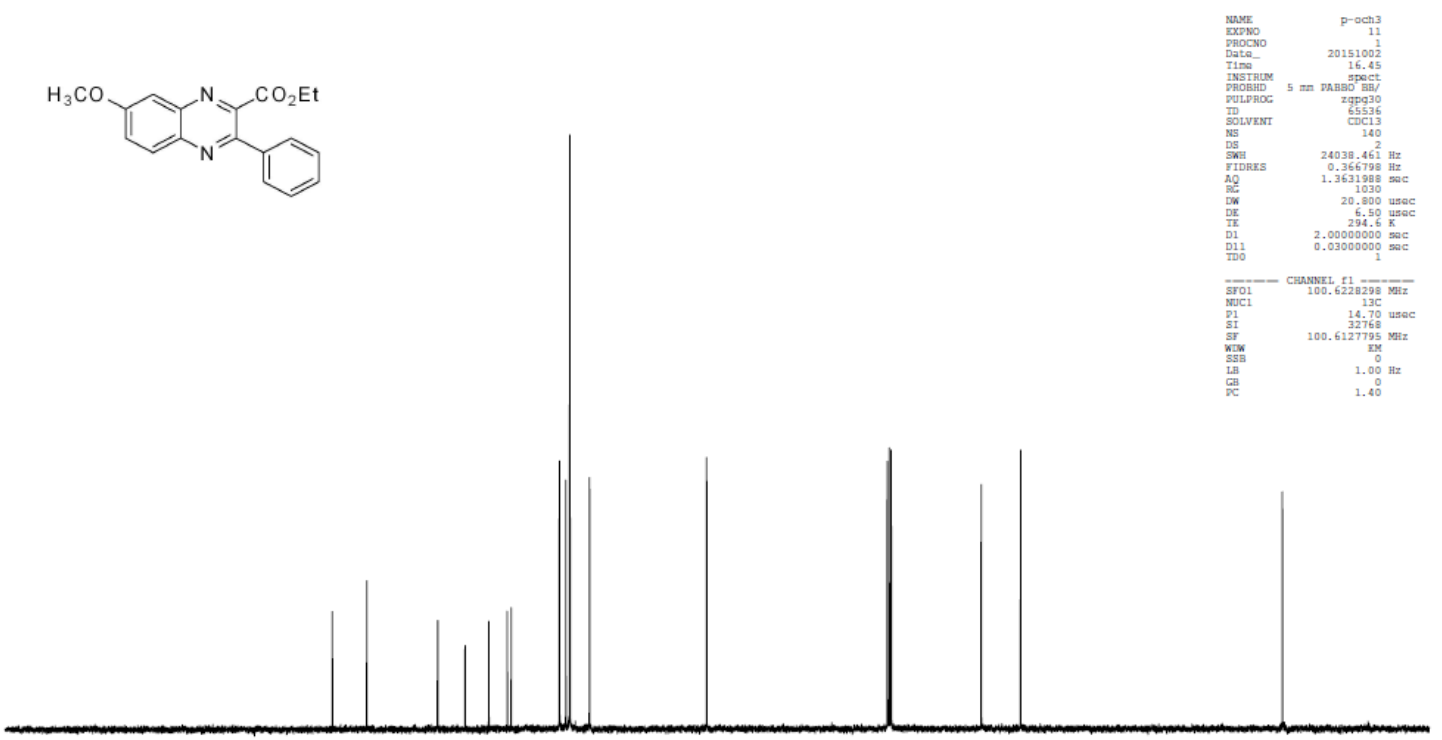

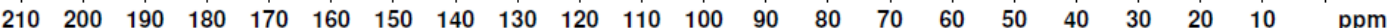




\section{2d}
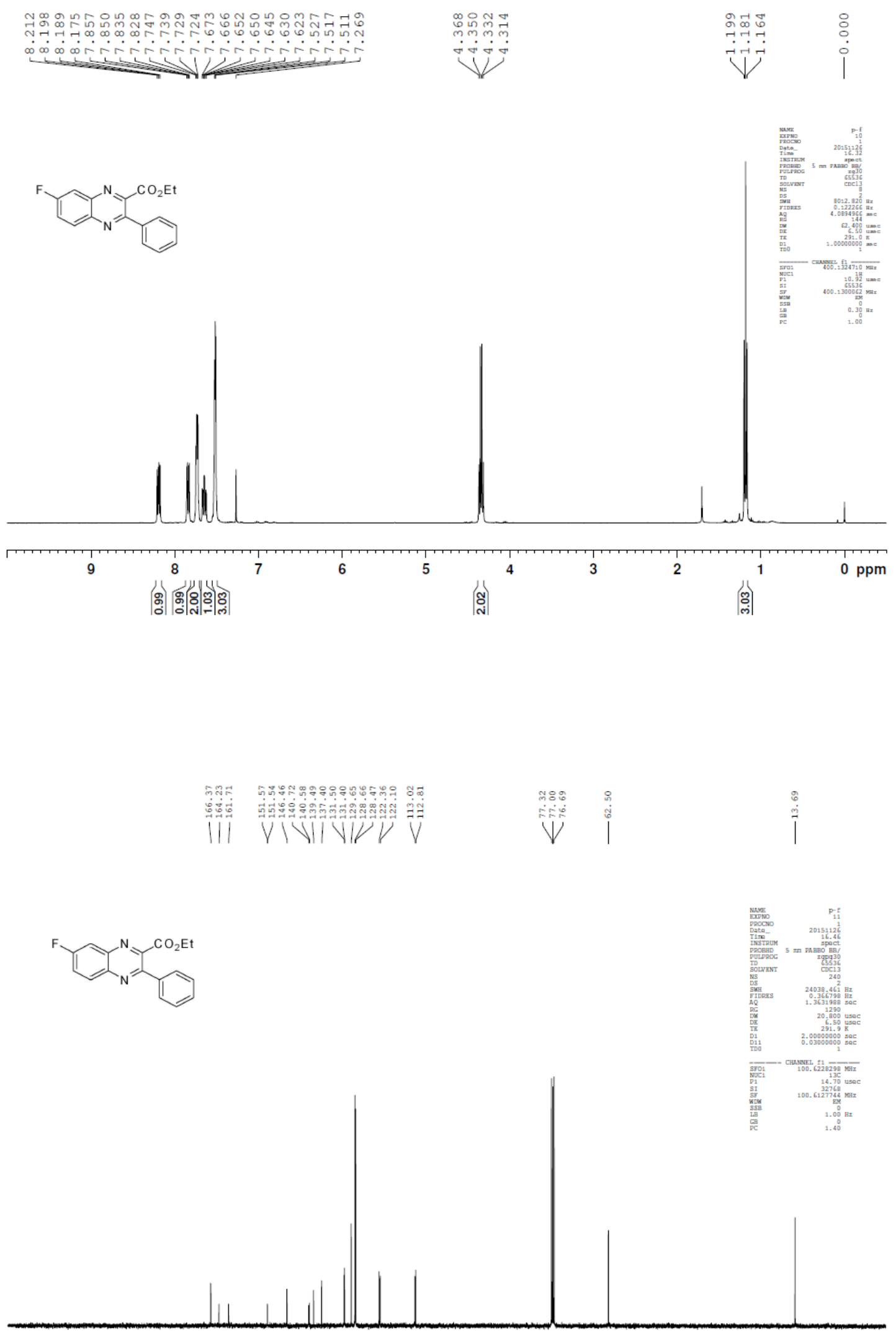


\section{$2 e$}

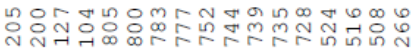

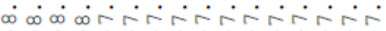

ज्ञल्लिख्ले

-

$\Rightarrow a$

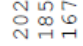

نें

:<smiles>CCOC(=O)c1nc2cc(C)ccc2nc1-c1ccccc1</smiles>
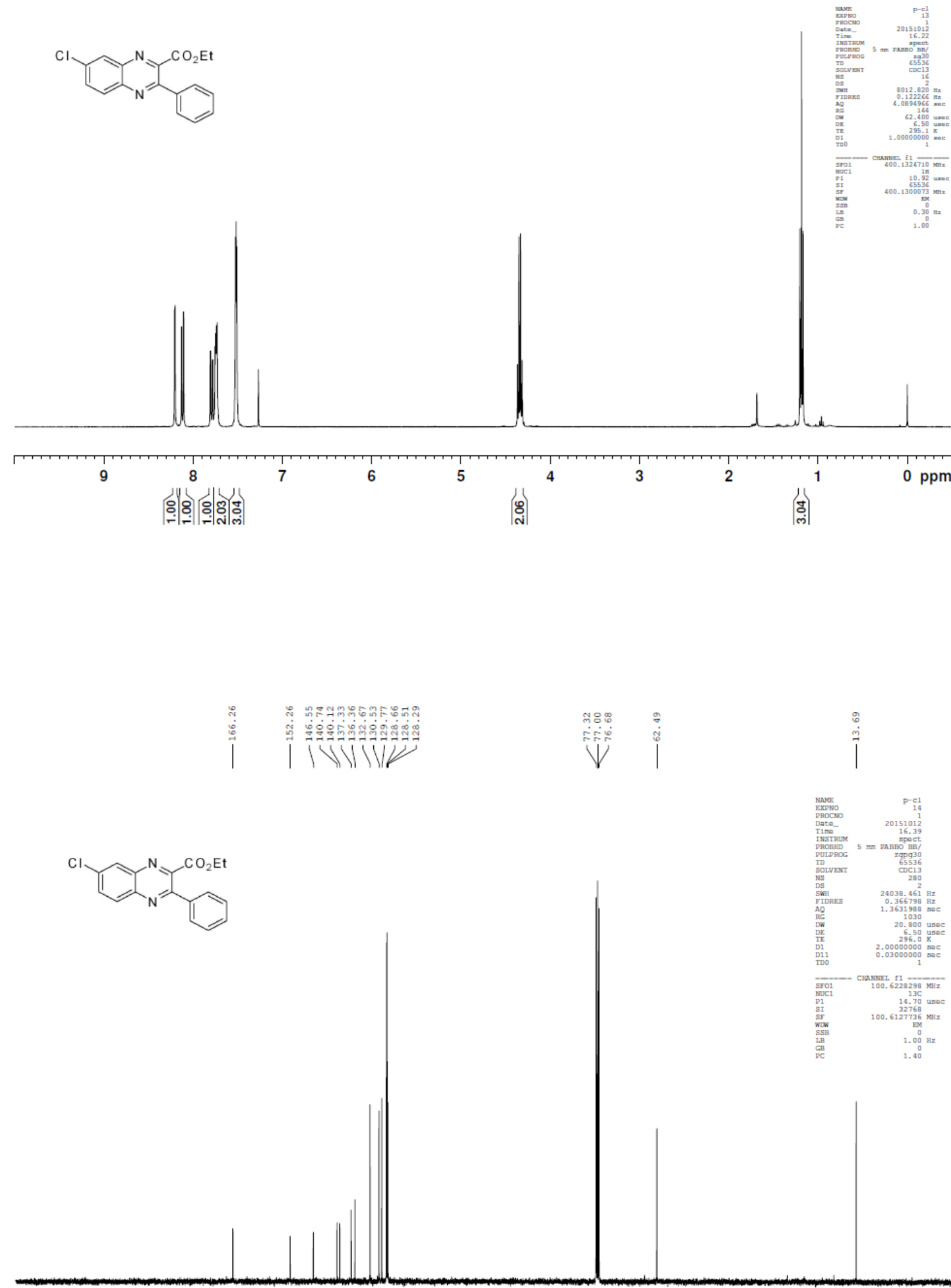

$\begin{array}{llllllllllllllllllllll}210 & 200 & 190 & 180 & 170 & 160 & 150 & 140 & 130 & 120 & 110 & 100 & 90 & 80 & 70 & 60 & 50 & 40 & 30 & 20 & 10 & \mathrm{ppm}\end{array}$ 
$2 f$
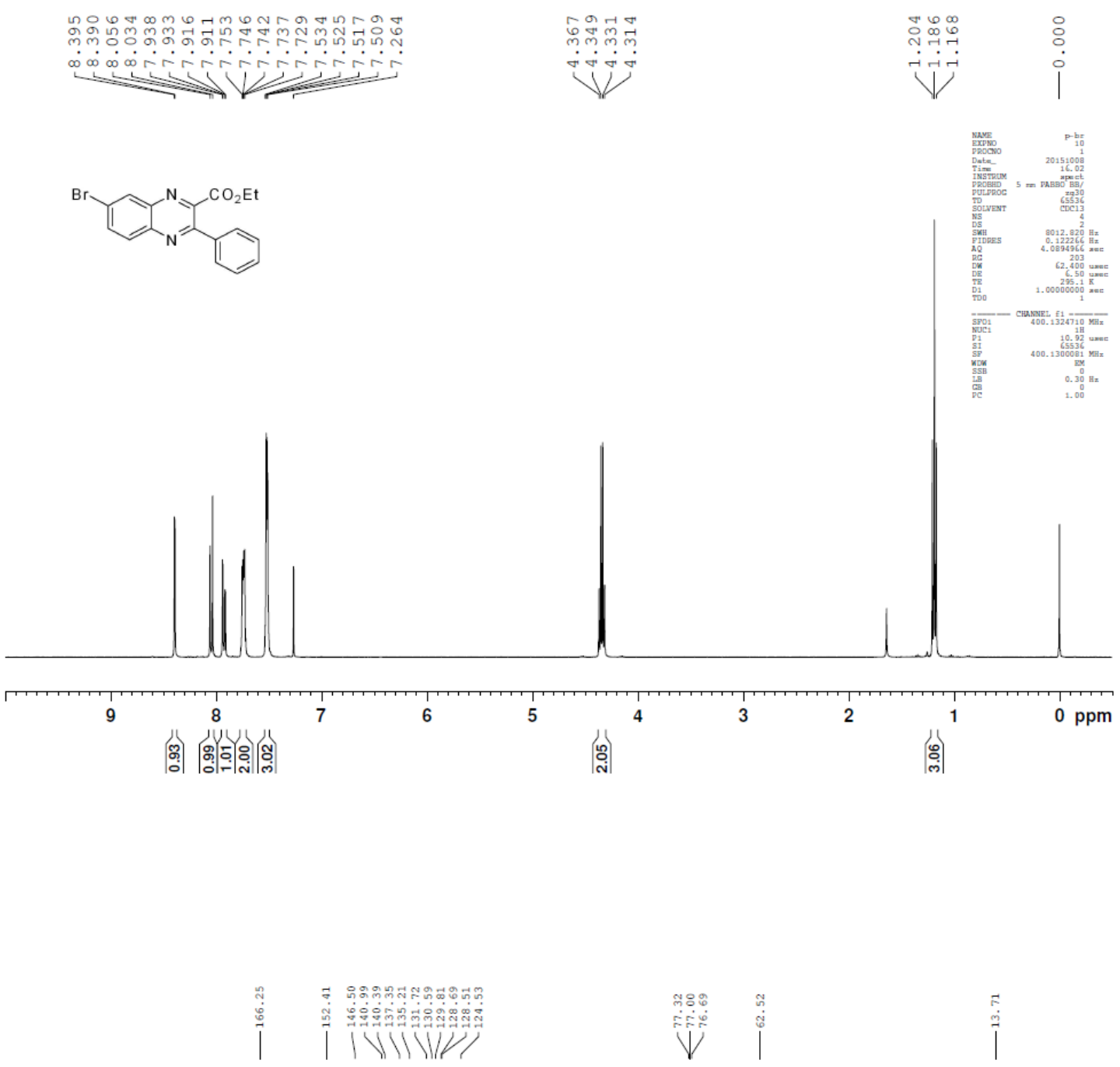

$\stackrel{\vec{n}}{\overrightarrow{3}}$
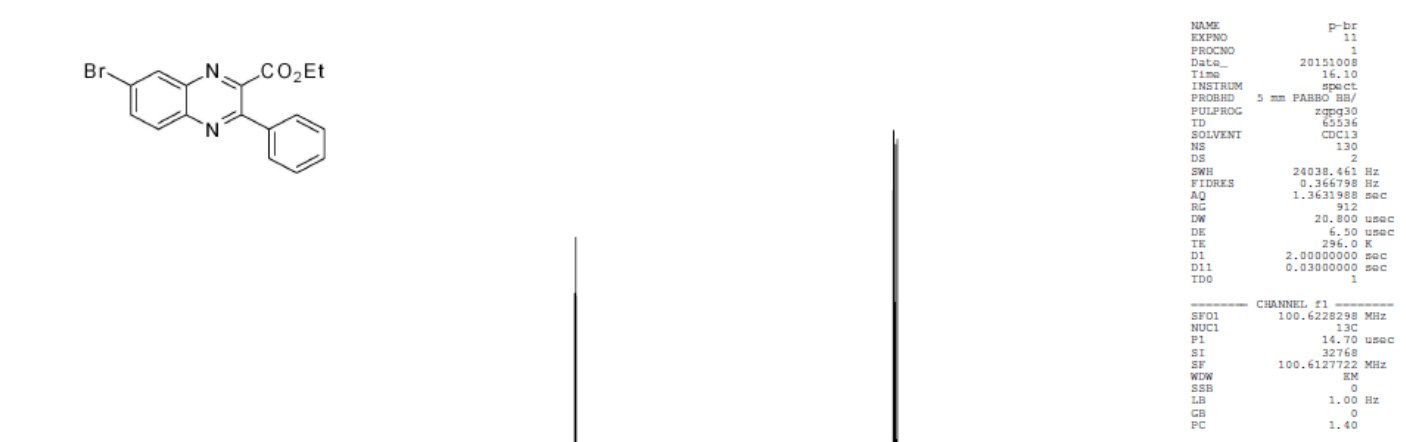

$\begin{array}{lllllllllllllllllllll}210 & 200 & 190 & 180 & 170 & 160 & 150 & 140 & 130 & 120 & 110 & 100 & 90 & 80 & 70 & 60 & 50 & 40 & 30 & 20 & 10\end{array}$ 
$2 \mathrm{~g}$

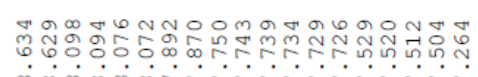

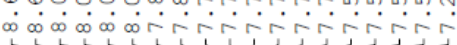

क्लेंग्ले

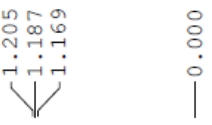

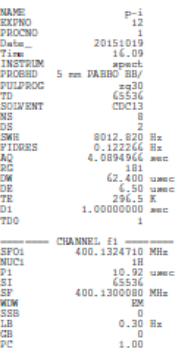

(1)
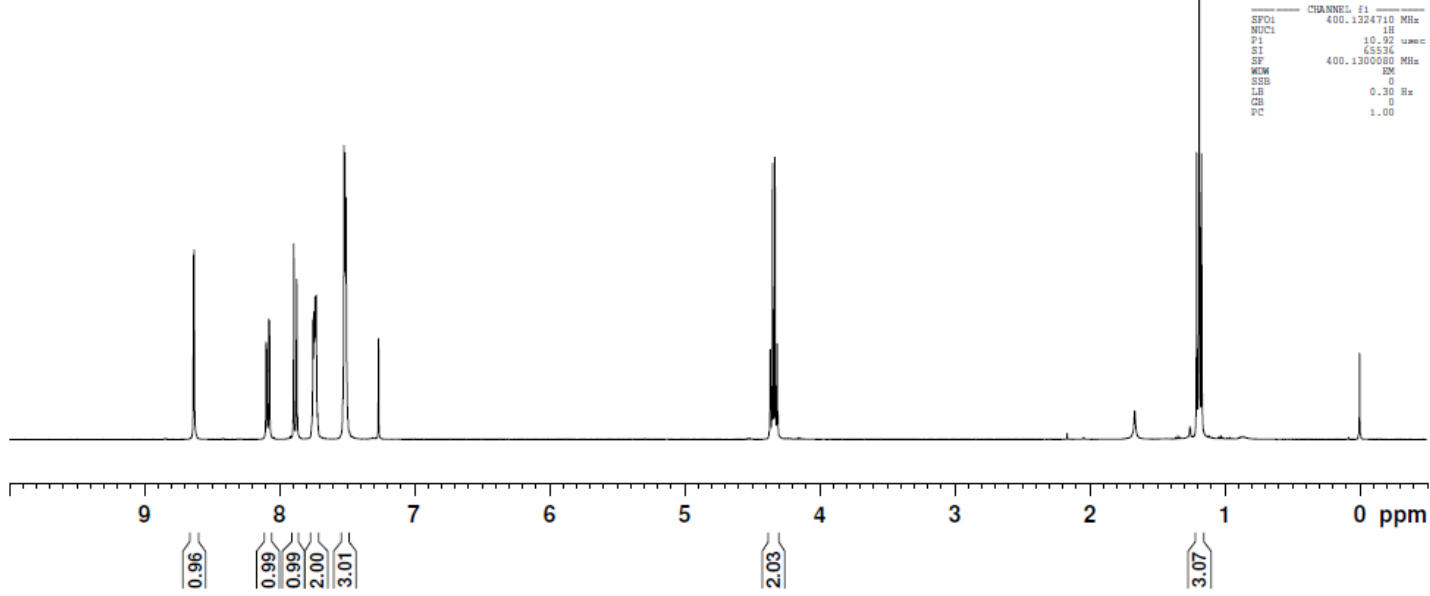

: 品通|

(ู)

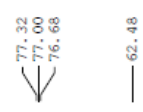

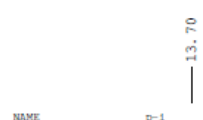
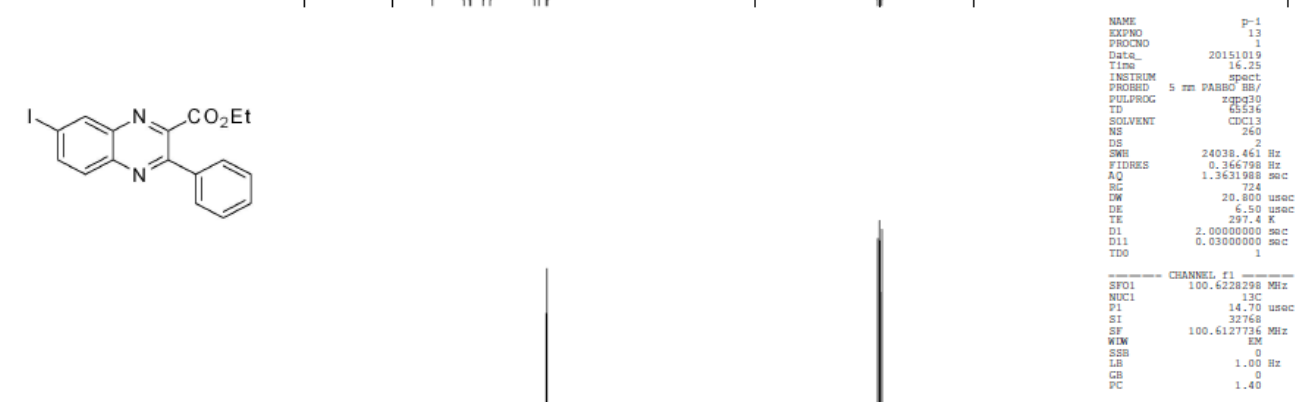

$$
\mid
$$
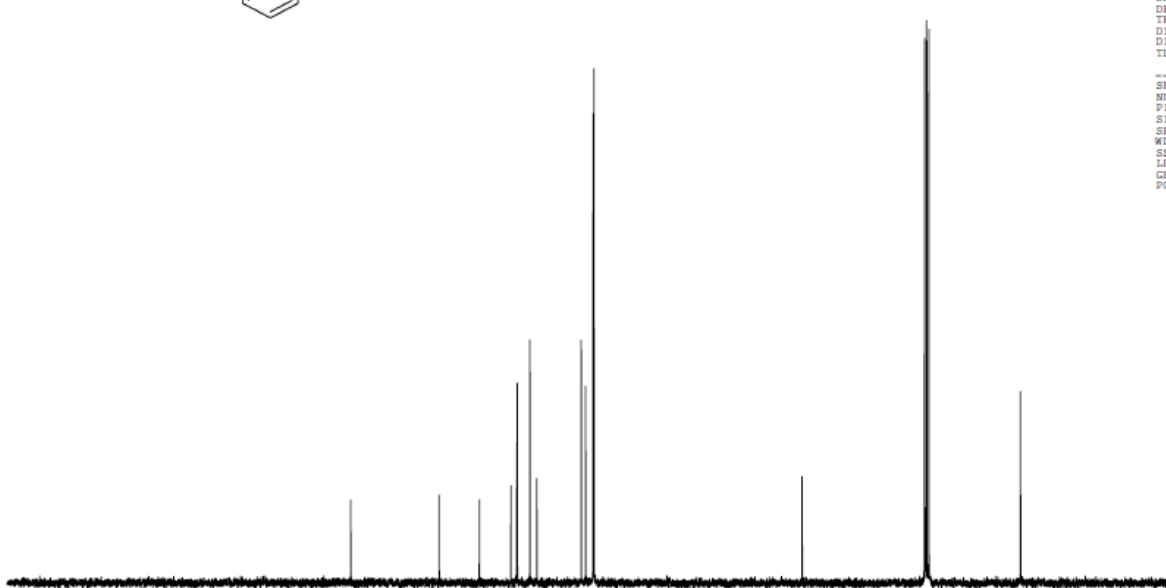

S $\begin{array}{lllllllllllllllllllll}210 & 200 & 190 & 180 & 170 & 160 & 150 & 140 & 130 & 120 & 110 & 100 & 90 & 80 & 70 & 60 & 50 & 40 & 30 & 20 & \mathrm{ppm}\end{array}$ 
$2 h$

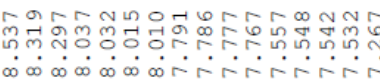

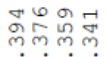

$+\infty$

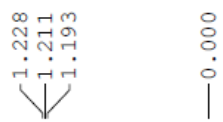

(1)
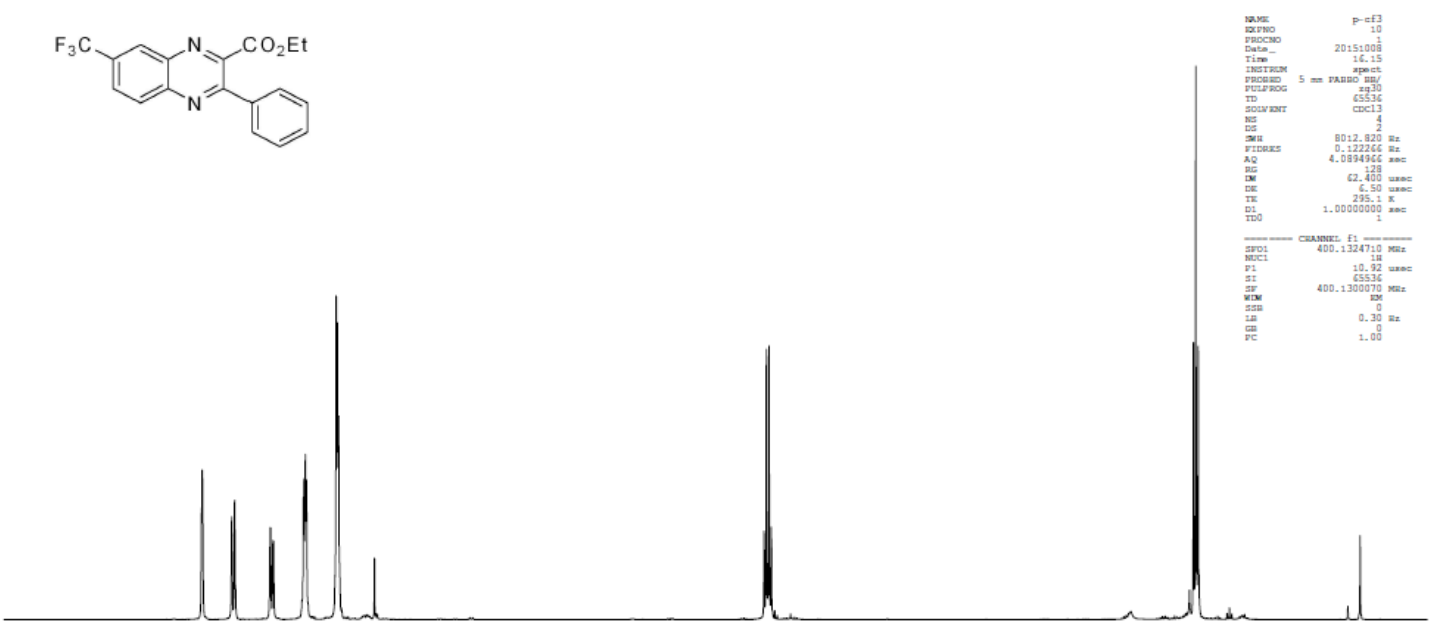

9

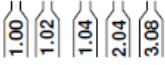

|

1

0 ppm

लें

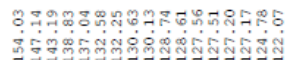

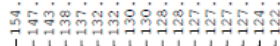

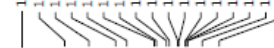
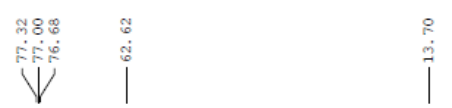

(1)

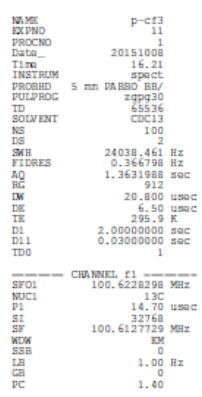

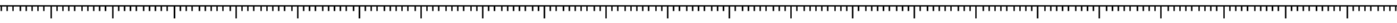

$\begin{array}{lllllllllllllllllllll}210 & 200 & 190 & 180 & 170 & 160 & 150 & 140 & 130 & 120 & 110 & 100 & 90 & 80 & 70 & 60 & 50 & 40 & 30 & 20 & 10\end{array}$ 
$2 \mathrm{i}$

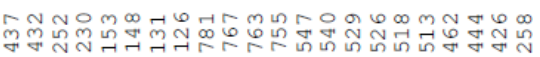

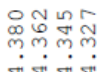

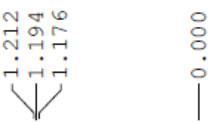

2
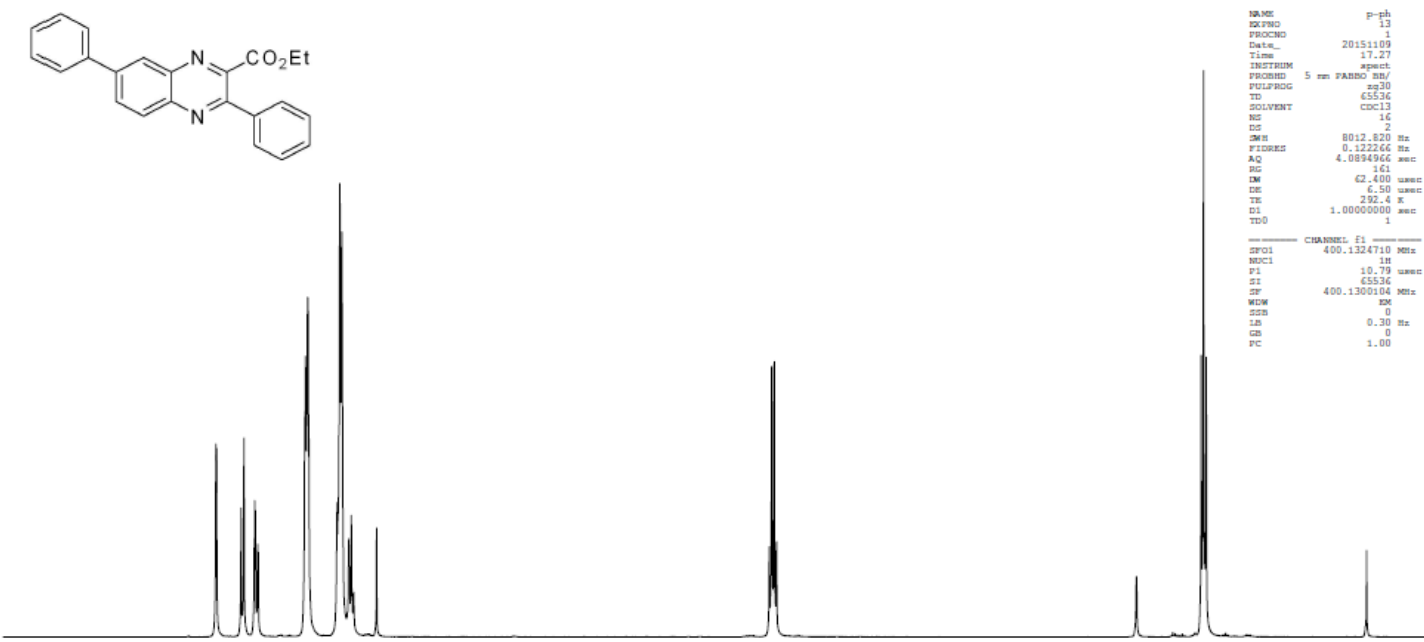
या1

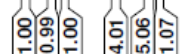

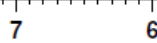

65
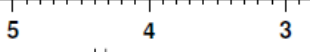

ma

1

ర్ల్

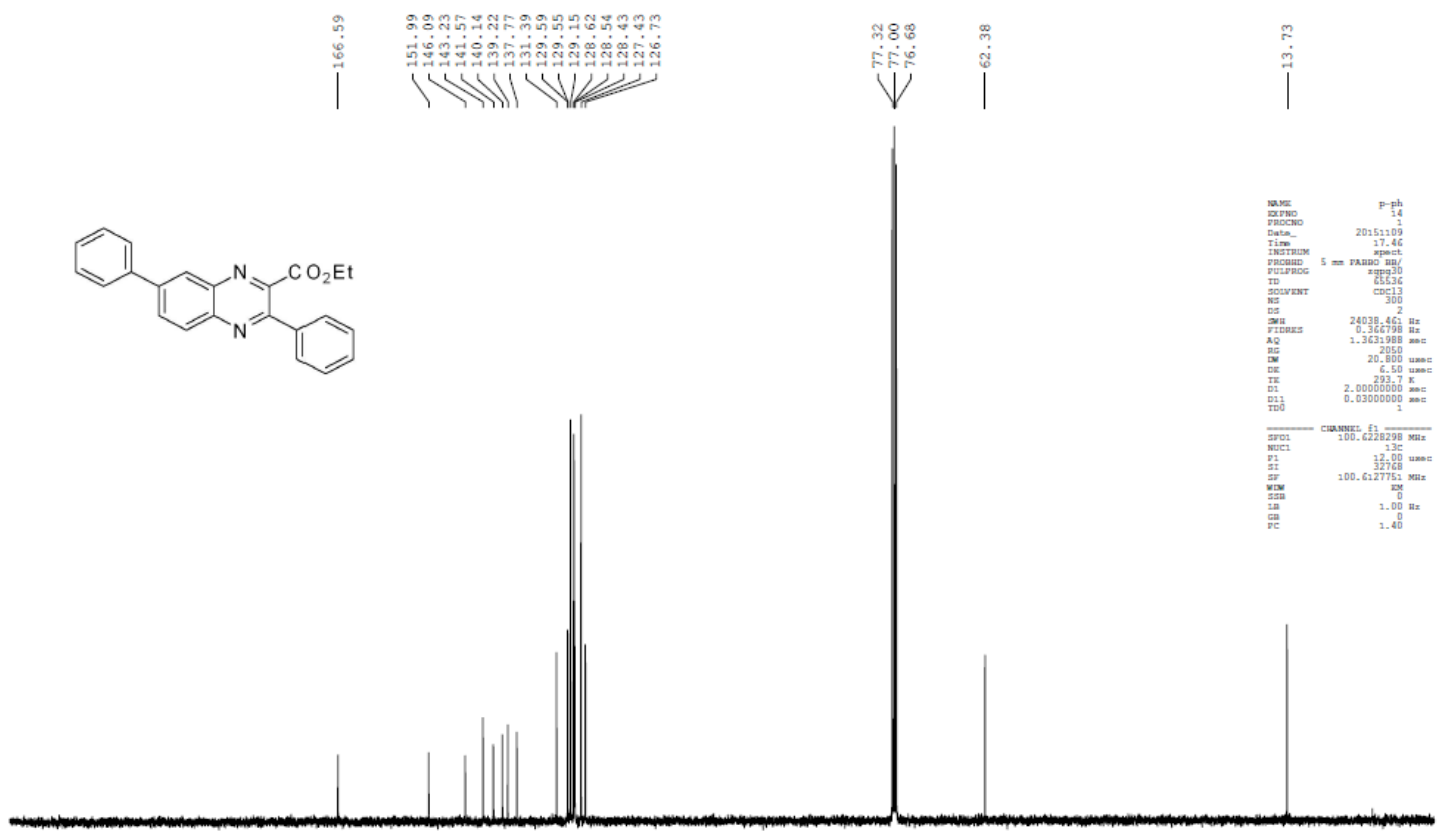

$\begin{array}{llllllllllllllllllllll}210 & 200 & 190 & 180 & 170 & 160 & 150 & 140 & 130 & 120 & 110 & 100 & 90 & 80 & 70 & 60 & 50 & 40 & 30 & 20 & 10 & \mathrm{ppm}\end{array}$ 


\section{2j-1}
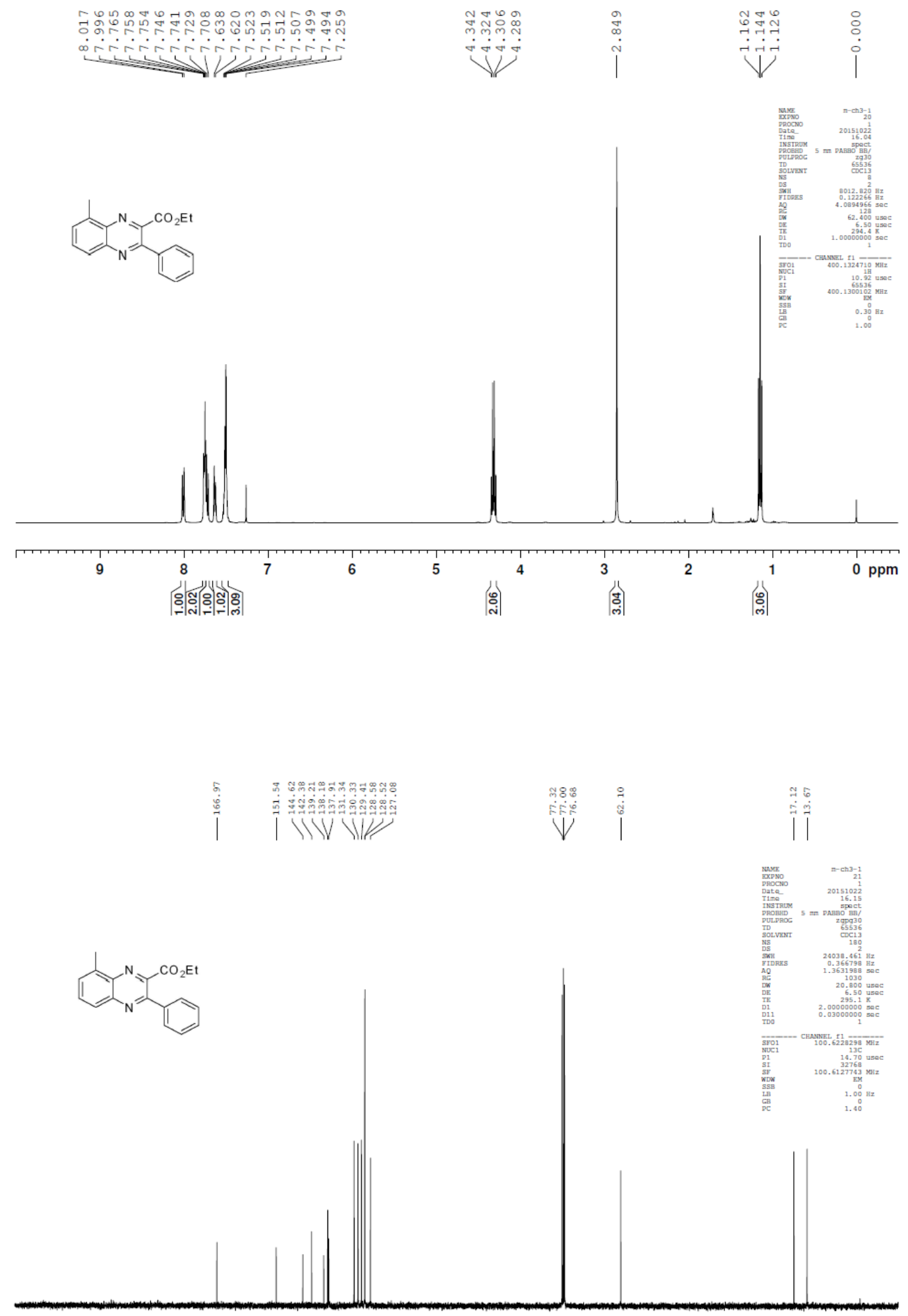

$\begin{array}{llllllllllllllllllllll}210 & 200 & 190 & 180 & 170 & 160 & 150 & 140 & 130 & 120 & 110 & 100 & 90 & 80 & 70 & 60 & 50 & 40 & 30 & 20 & 10 & \mathrm{ppm}\end{array}$ 


\section{2j-2}

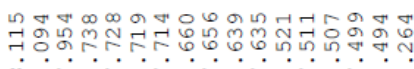

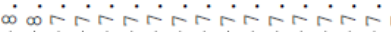

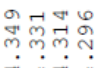

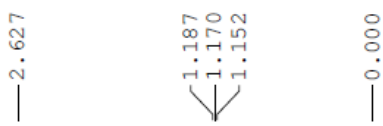

$\overbrace{\mathrm{N}}^{\mathrm{N}} \mathrm{Y}^{\mathrm{CO}_{2} \mathrm{Et}}$
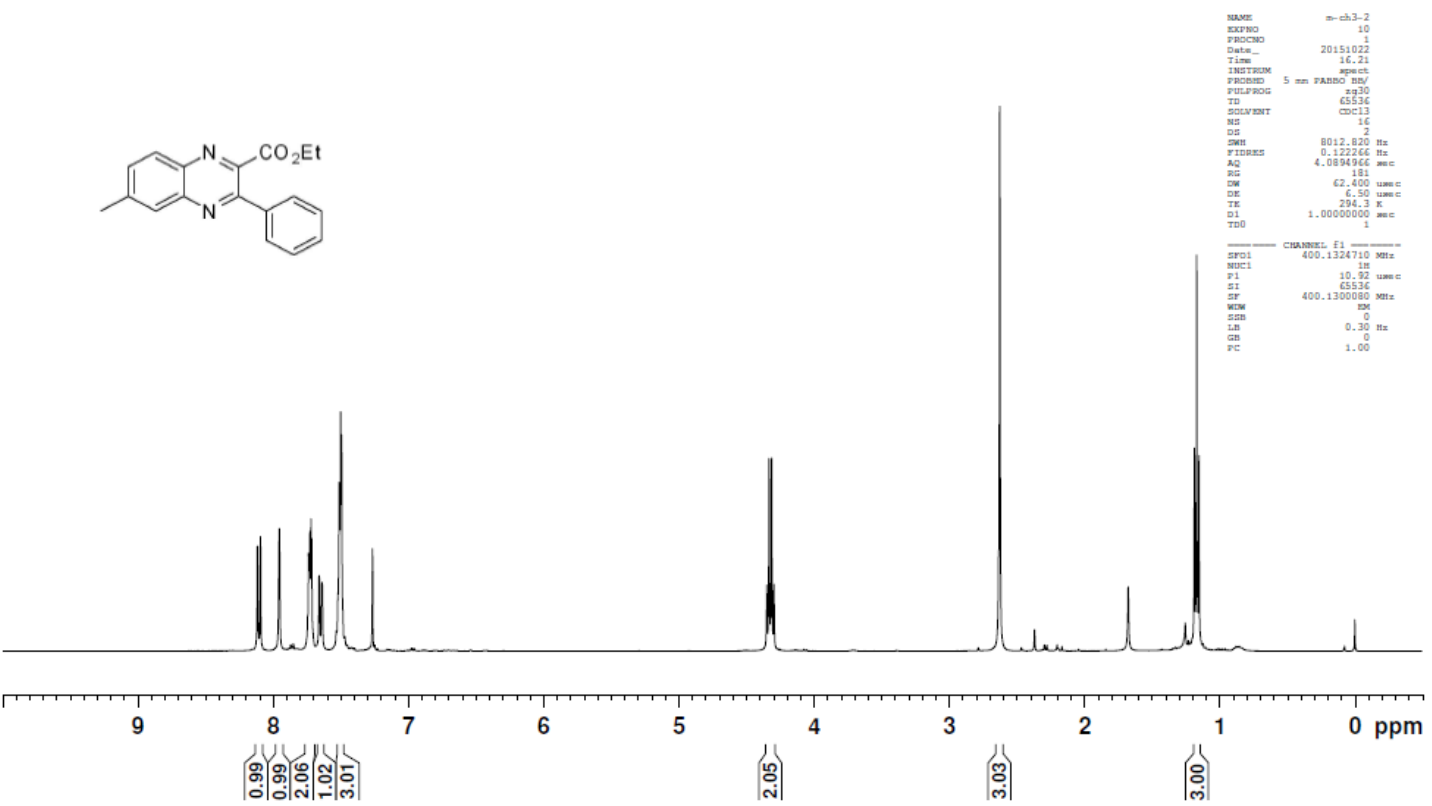

$\overbrace{N}^{\mathrm{N}} \mathrm{CO}_{\mathrm{N}}^{\mathrm{CO}_{2} \mathrm{Et}}$
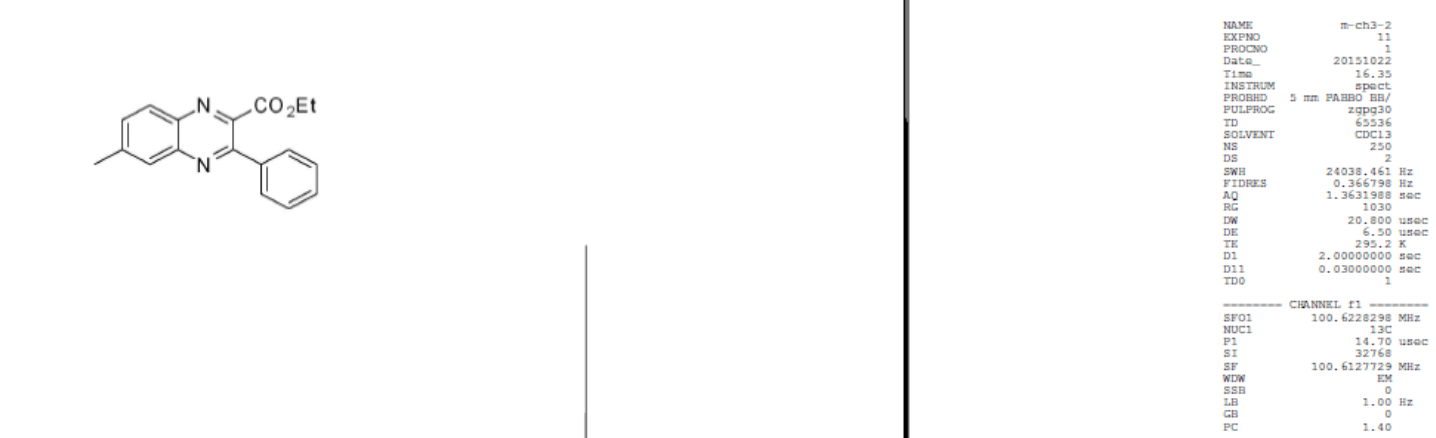

$\begin{array}{llllllllllllllllllllll}210 & 200 & 190 & 180 & 170 & 160 & 150 & 140 & 130 & 120 & 110 & 100 & 90 & 80 & 70 & 60 & 50 & 40 & 30 & 20 & 10 & \mathrm{ppm}\end{array}$ 


\section{2k-1, 2k-2}

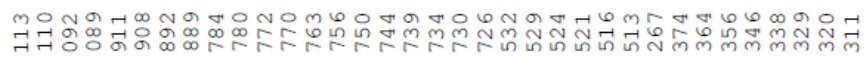

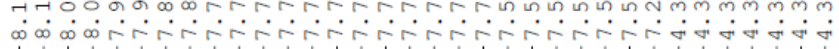

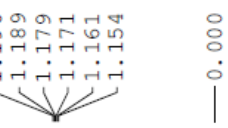

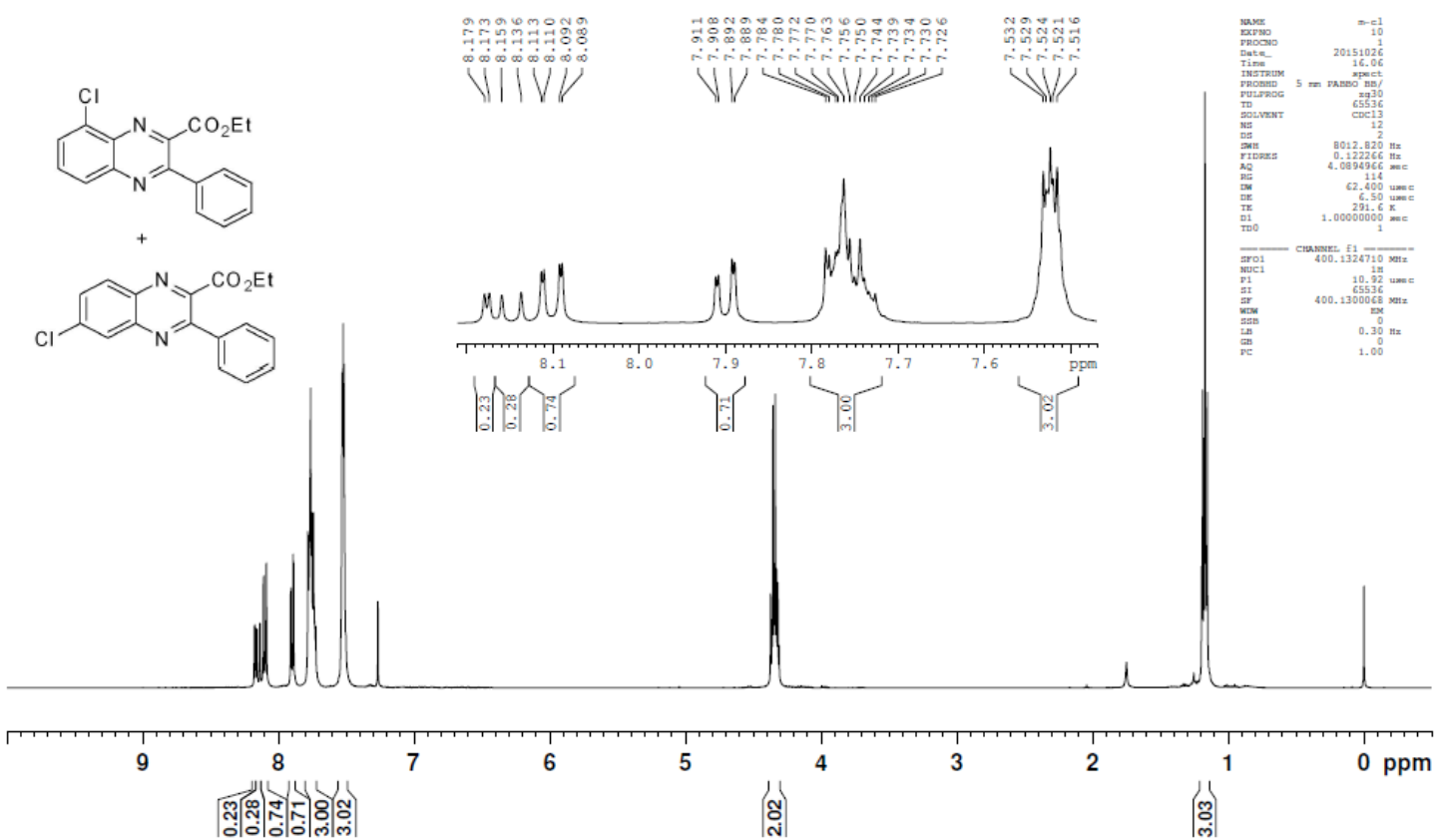

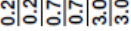

일

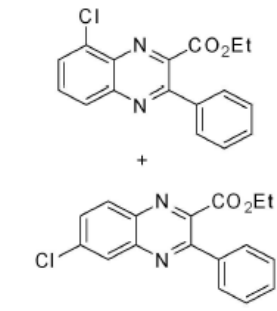

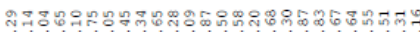

o

W

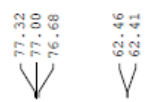

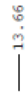
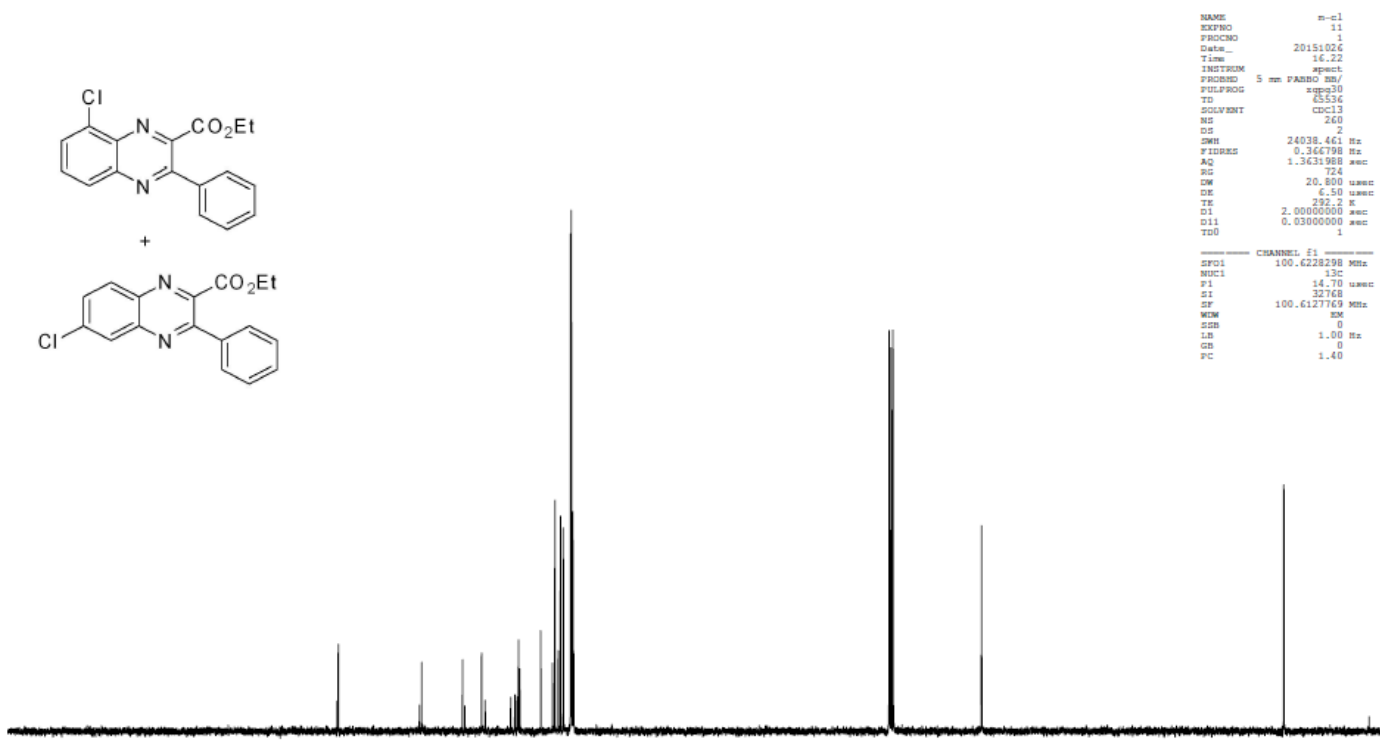

$\begin{array}{llllllllllllllllllllll}210 & 200 & 190 & 180 & 170 & 160 & 150 & 140 & 130 & 120 & 110 & 100 & 90 & 80 & 70 & 60 & 50 & 40 & 30 & 20 & 10 & \mathrm{ppm}\end{array}$ 
21

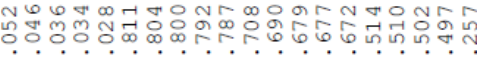

o mon on

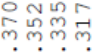

نே

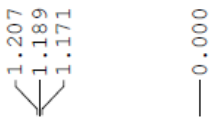

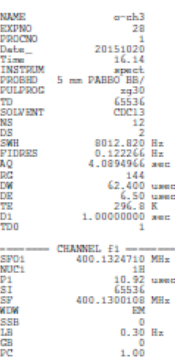

$\mathrm{C}^{\mathrm{N}}-\mathrm{CO}_{2} \mathrm{Et}$

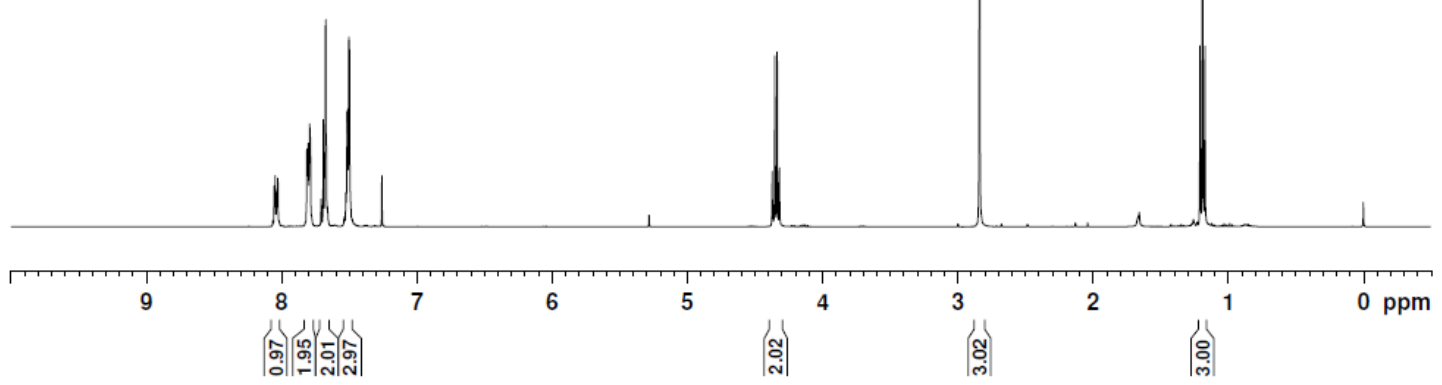

|

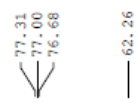

$\mid$

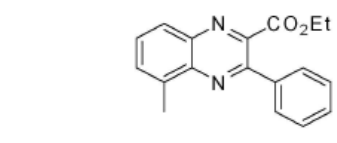

$\mathrm{O}_{2} \mathrm{Et}$
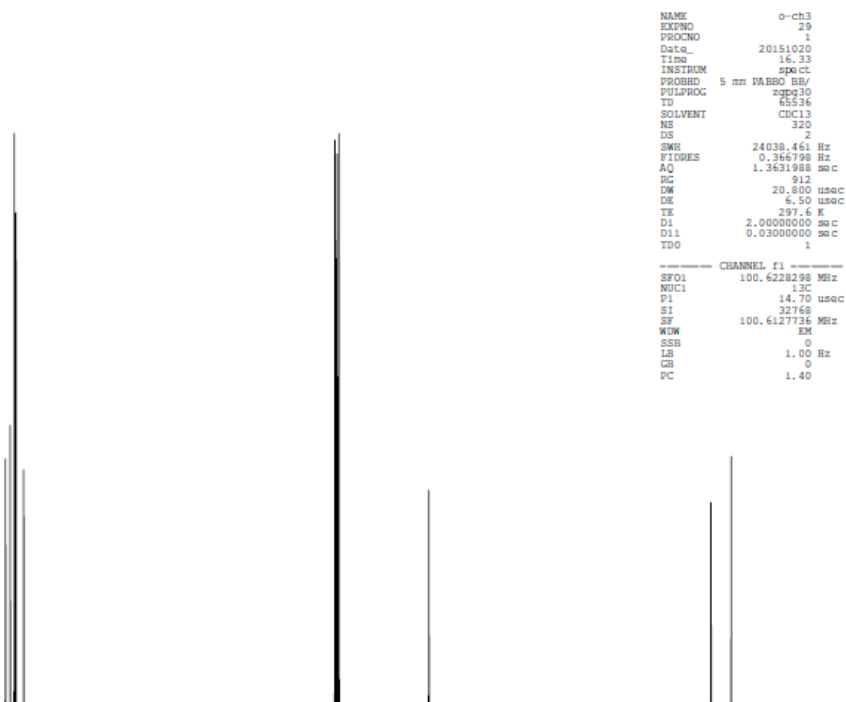

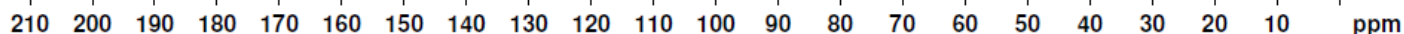


2n

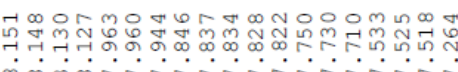

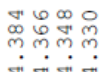

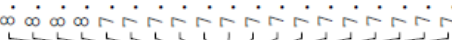

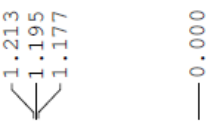

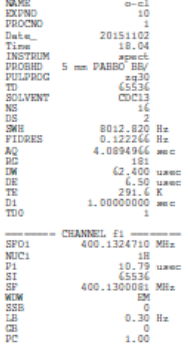

$\mathrm{Cl}^{\mathrm{N}} \mathrm{CO}^{\mathrm{CO} \mathrm{Et}}$

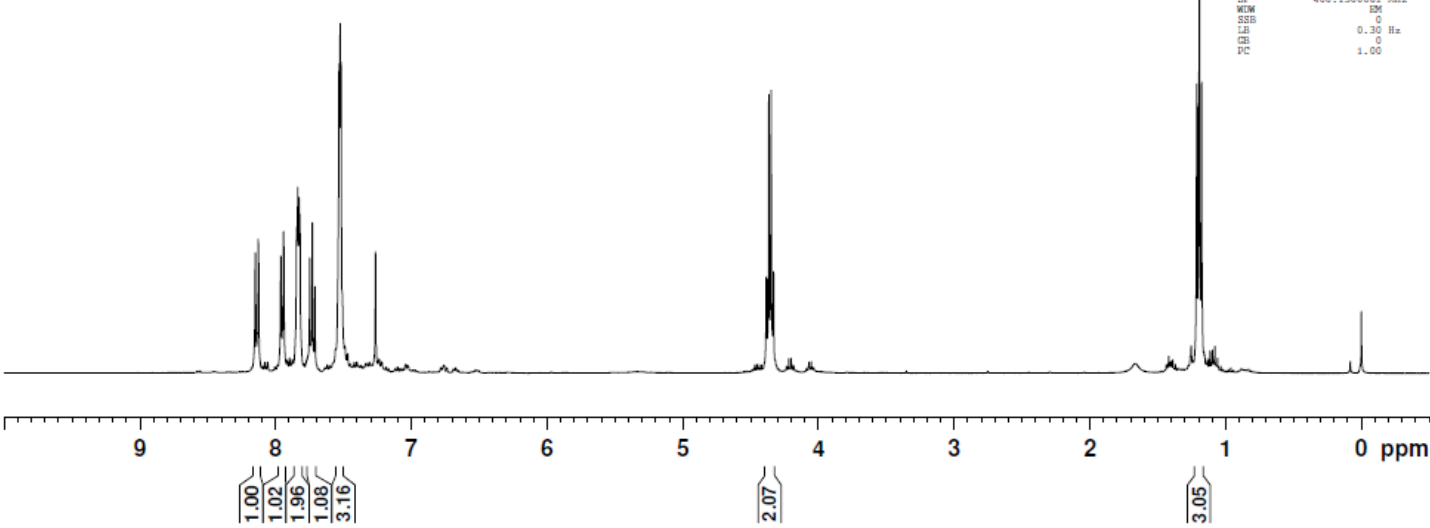

$\overbrace{\mathrm{Cl}}^{\mathrm{N}}=\mathrm{N}_{\mathrm{N}}^{\mathrm{CO}_{2} \mathrm{Et}}$

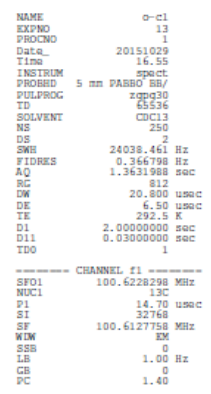

$\begin{array}{llllllllllllllllllllll}210 & 200 & 190 & 180 & 170 & 160 & 150 & 140 & 130 & 120 & 110 & 100 & 90 & 80 & 70 & 60 & 50 & 40 & 30 & 20 & 10 & \mathrm{ppm}\end{array}$ 


\section{0}

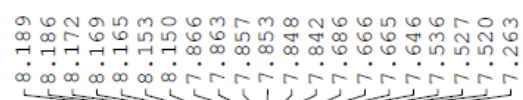

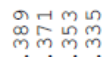

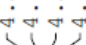

ฟin

${ }_{\mathrm{Br}}^{\mathrm{N}}$
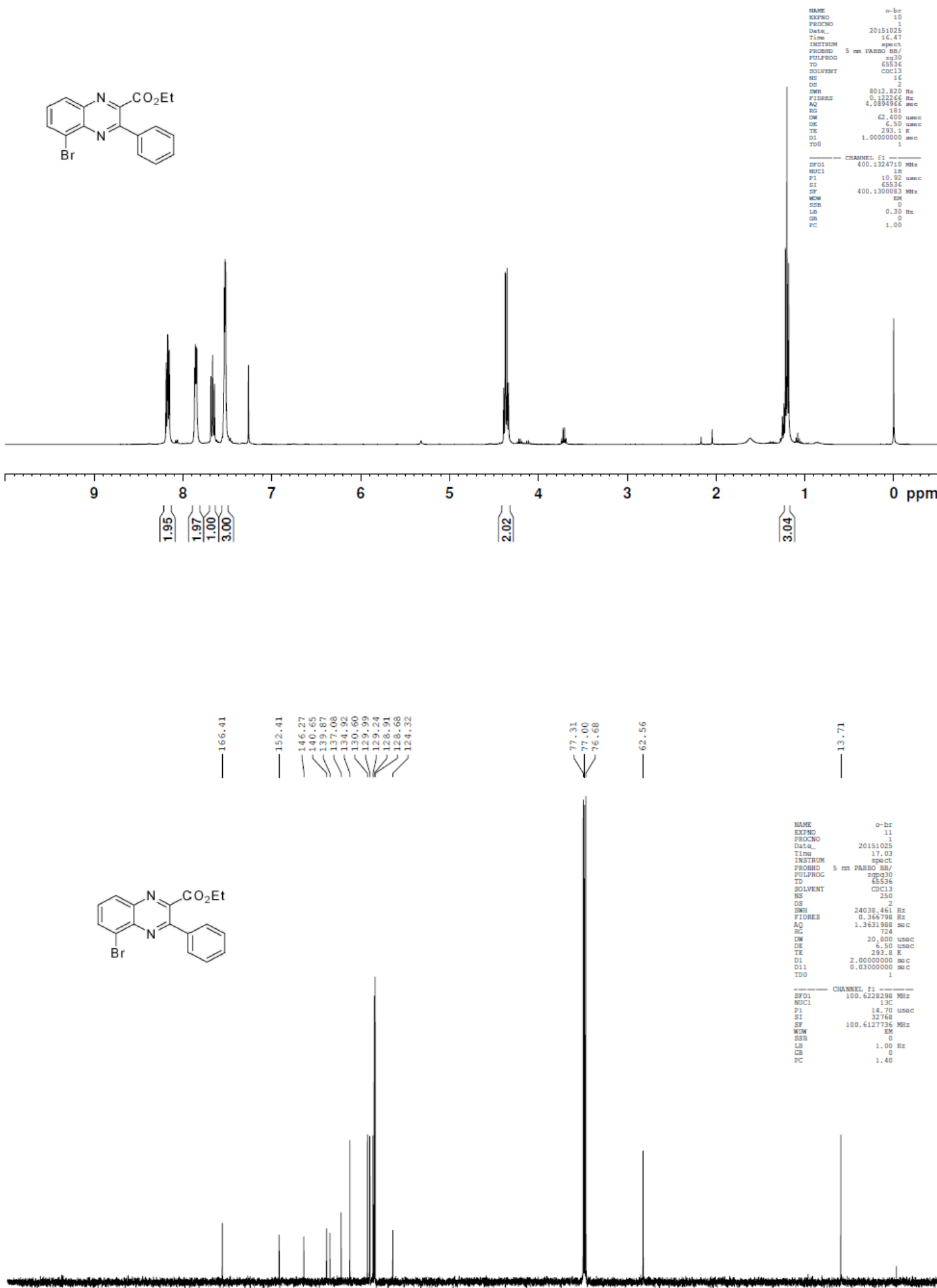

$\begin{array}{llllllllllllllllllllll}210 & 200 & 190 & 180 & 170 & 160 & 150 & 140 & 130 & 120 & 110 & 100 & 90 & 80 & 70 & 60 & 50 & 40 & 30 & 20 & 10 & \mathrm{ppm}\end{array}$ 
2p

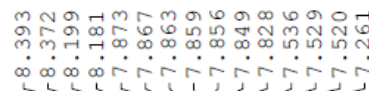

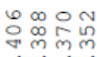

$\circ$
$\vdots$
$\vdots$<smiles>CCOC(=O)c1nc2cccc(C(F)(F)F)c2nc1-c1ccccc1</smiles>
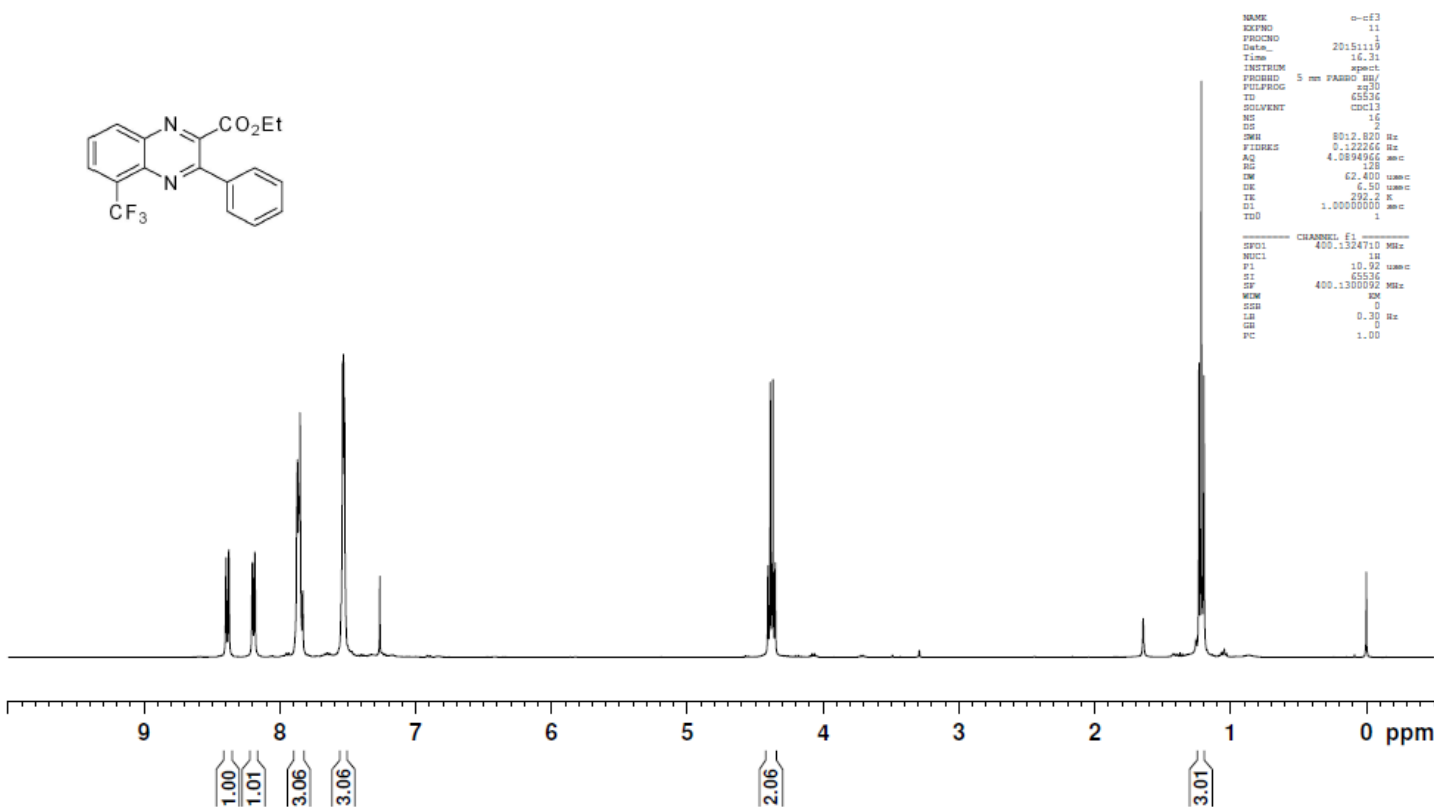

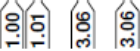

|ล่|

लें

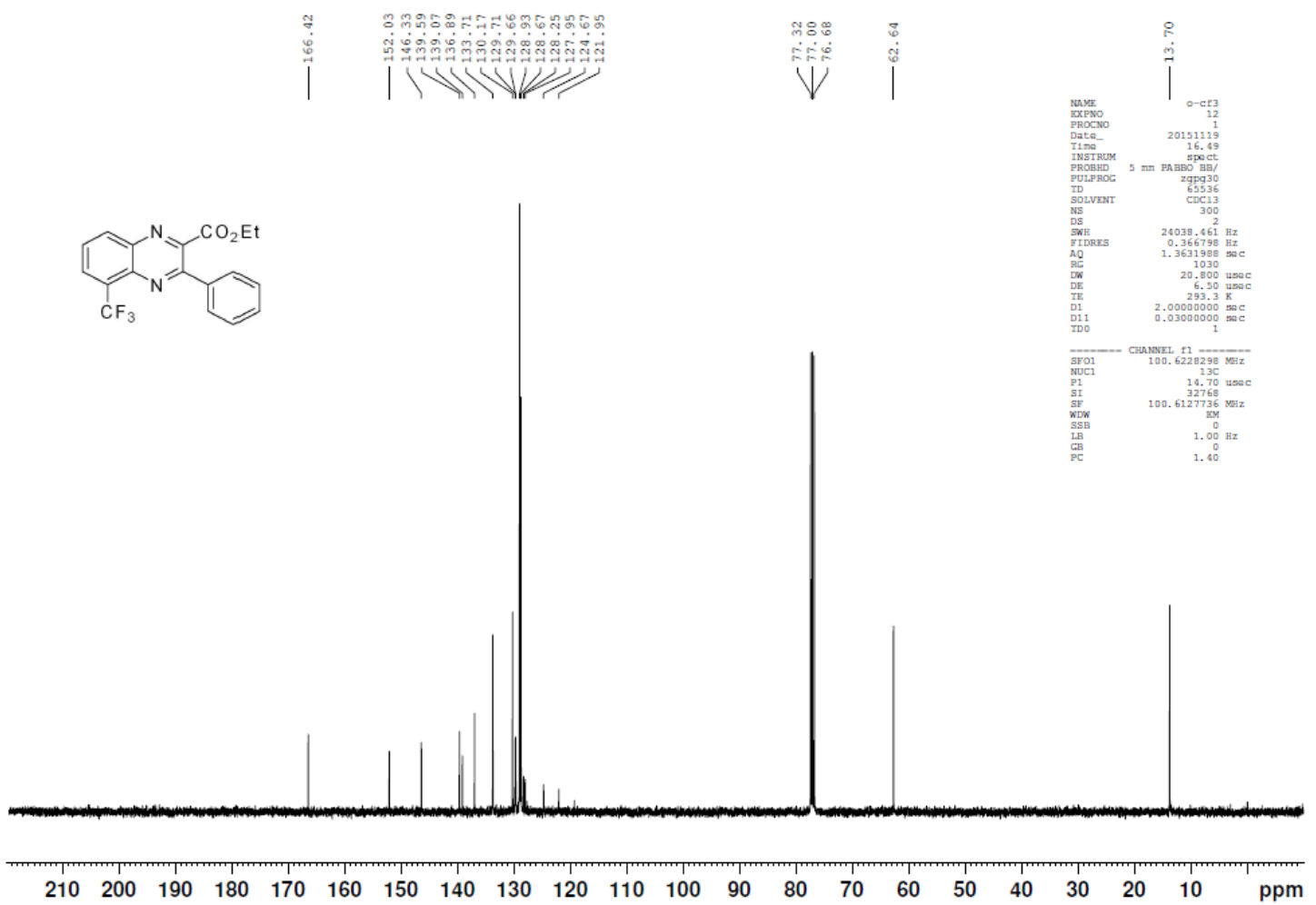


$2 q$

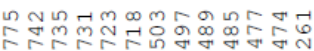

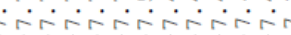

ำ 귀ำ

|

복ำ금

ரேர

:
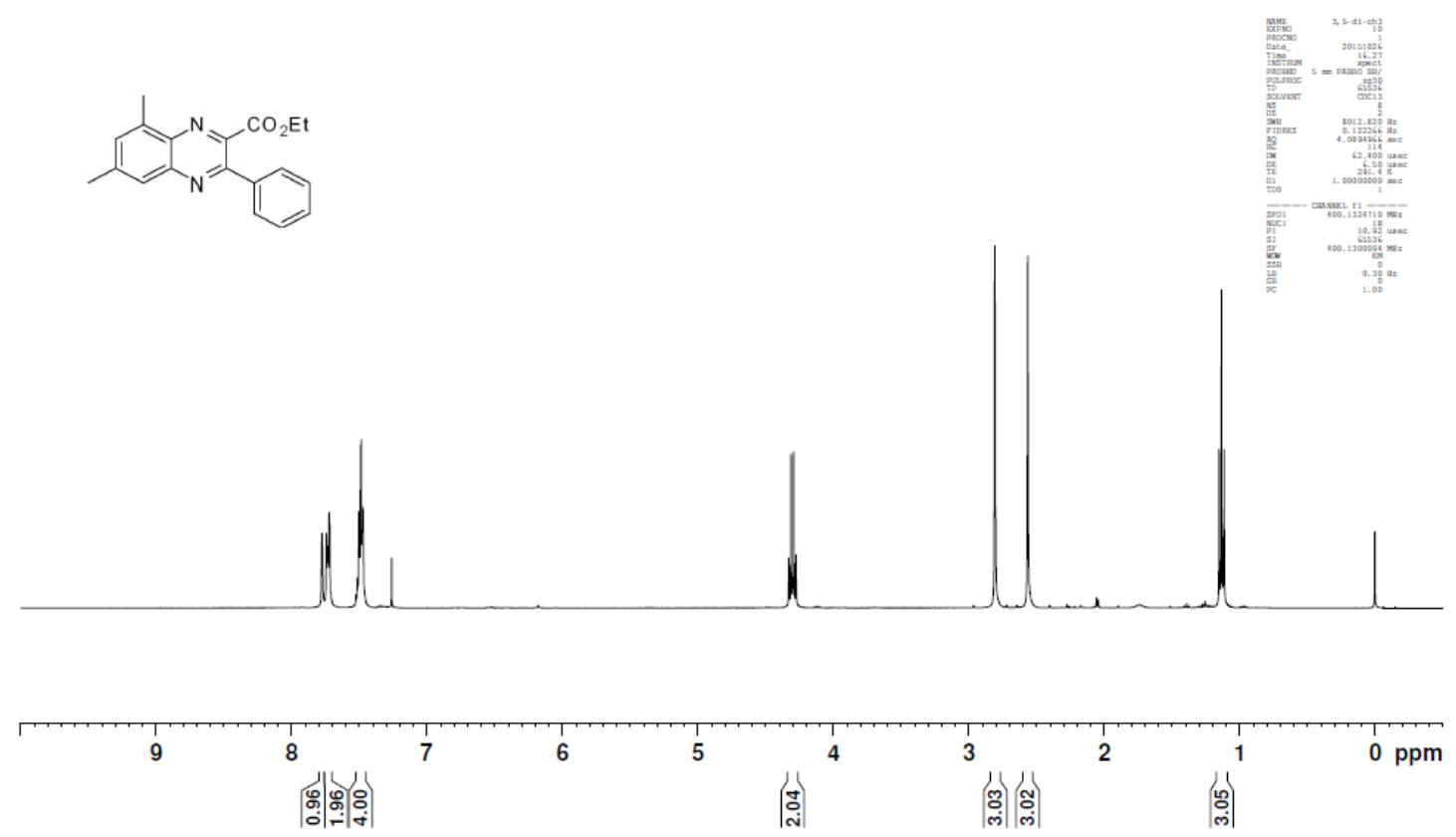

|

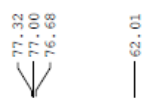

|ึ่
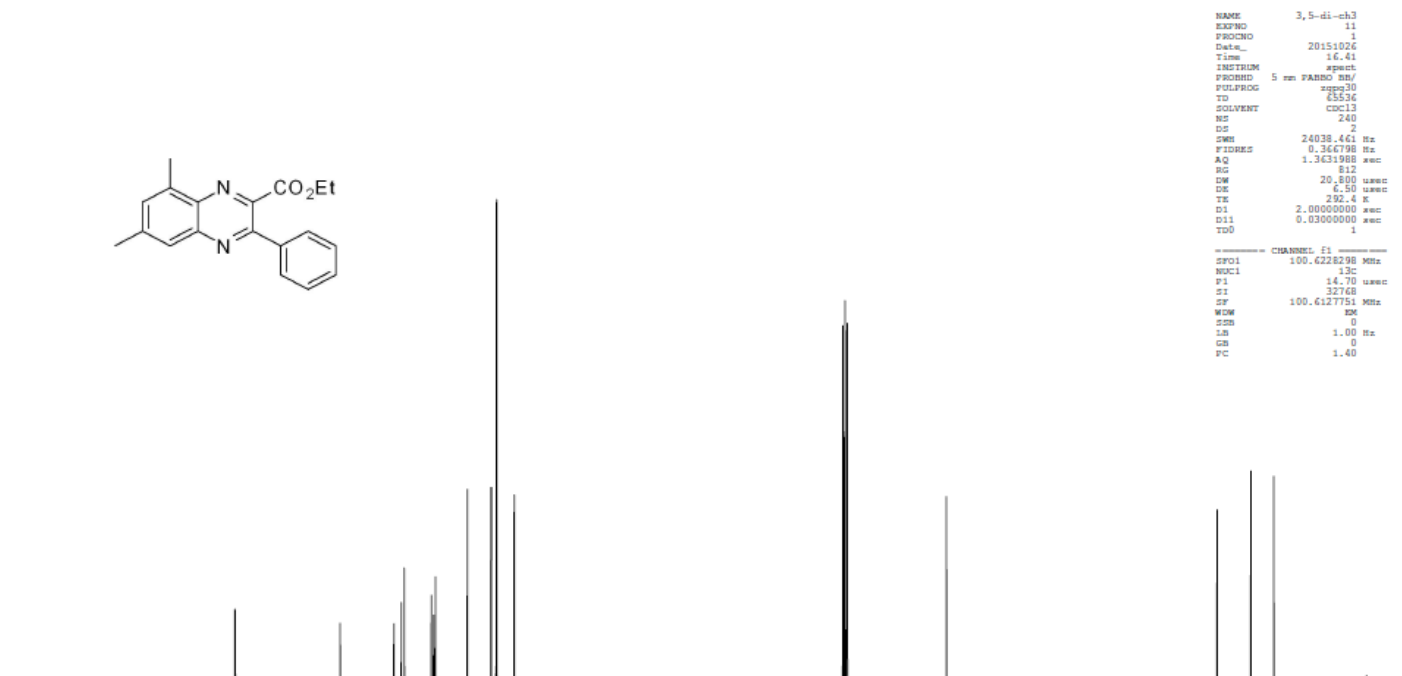

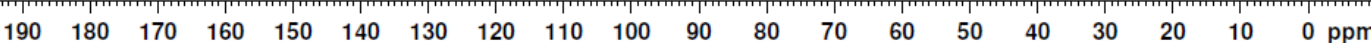




\section{2r}

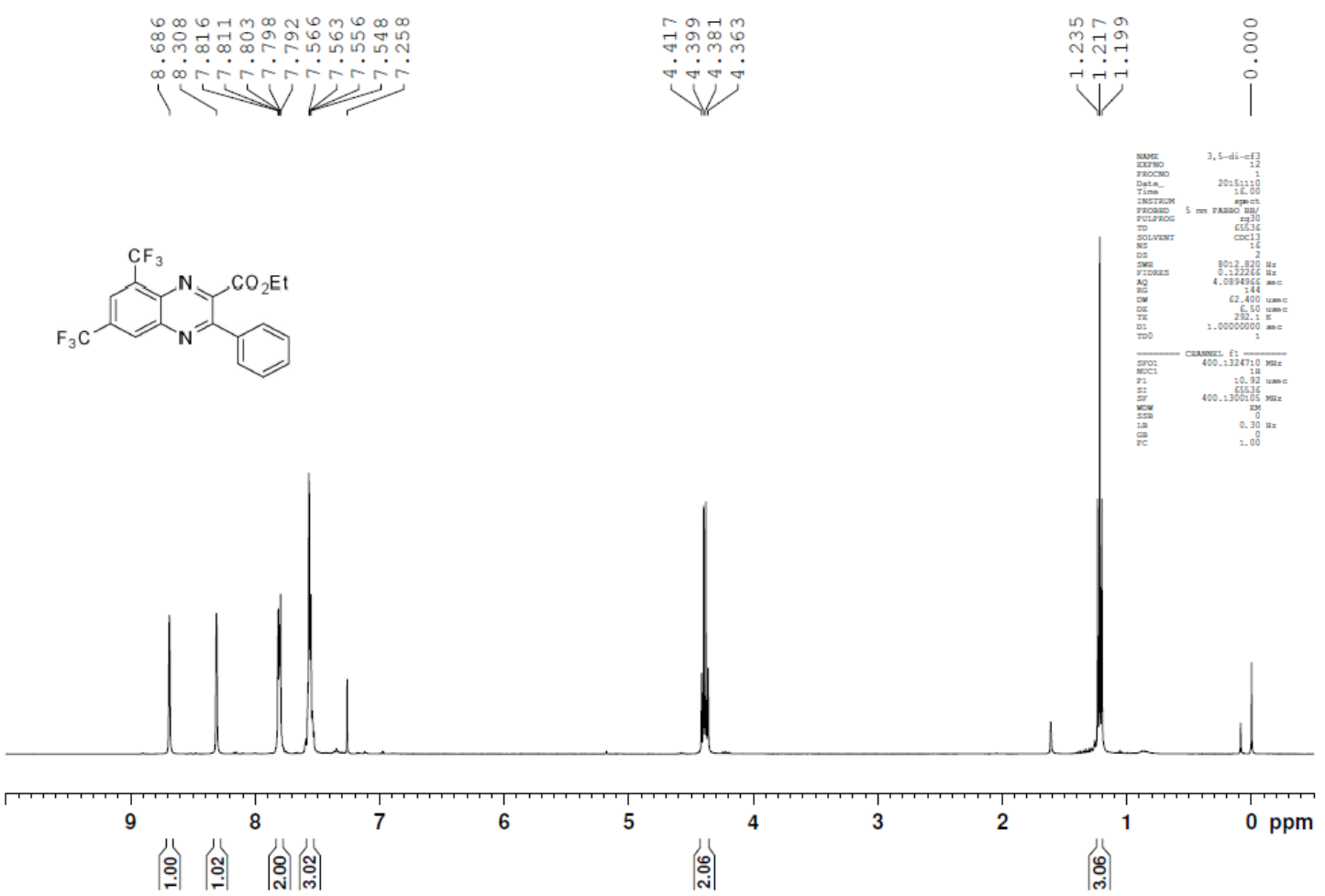

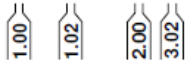

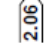

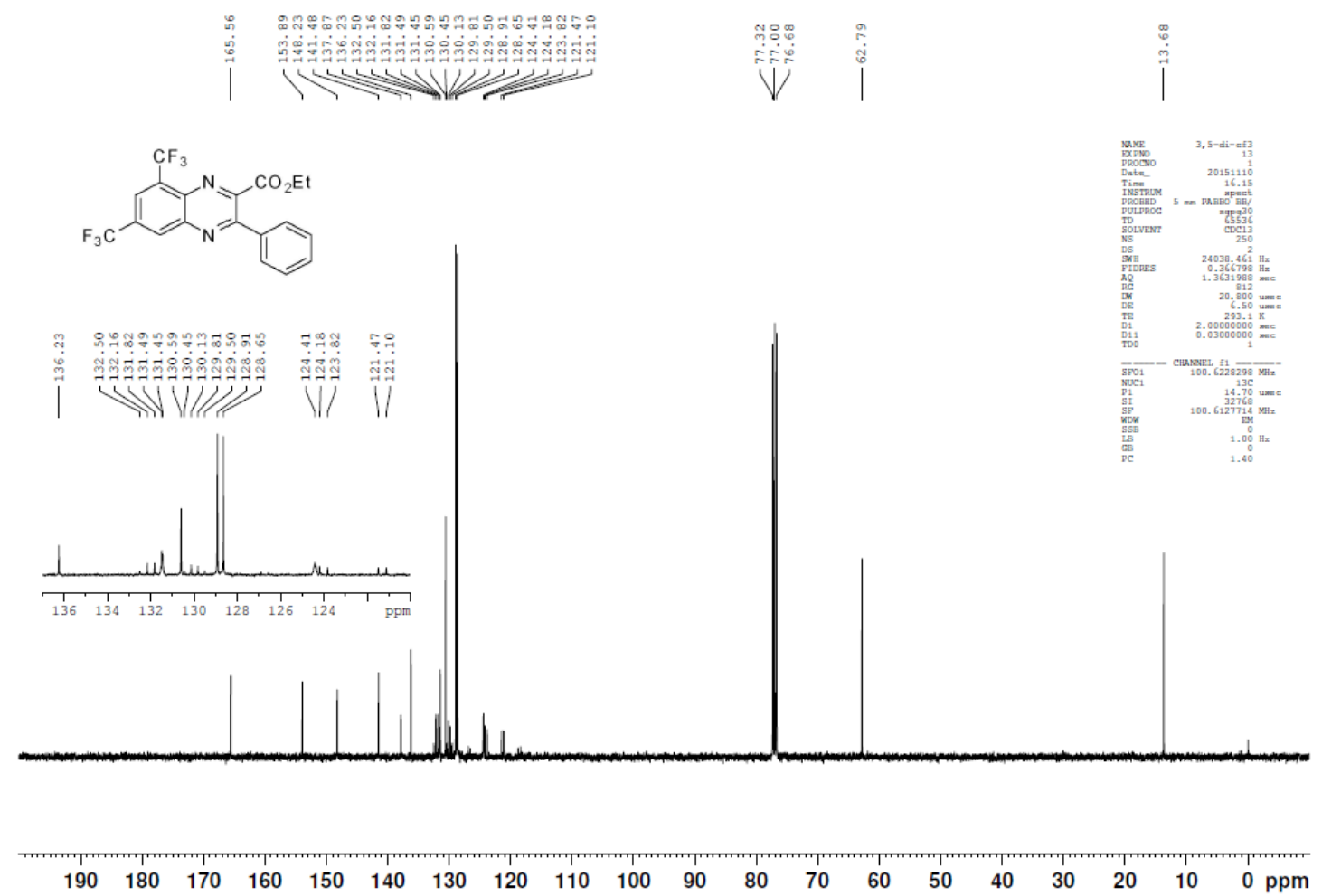




\section{$2 s$}
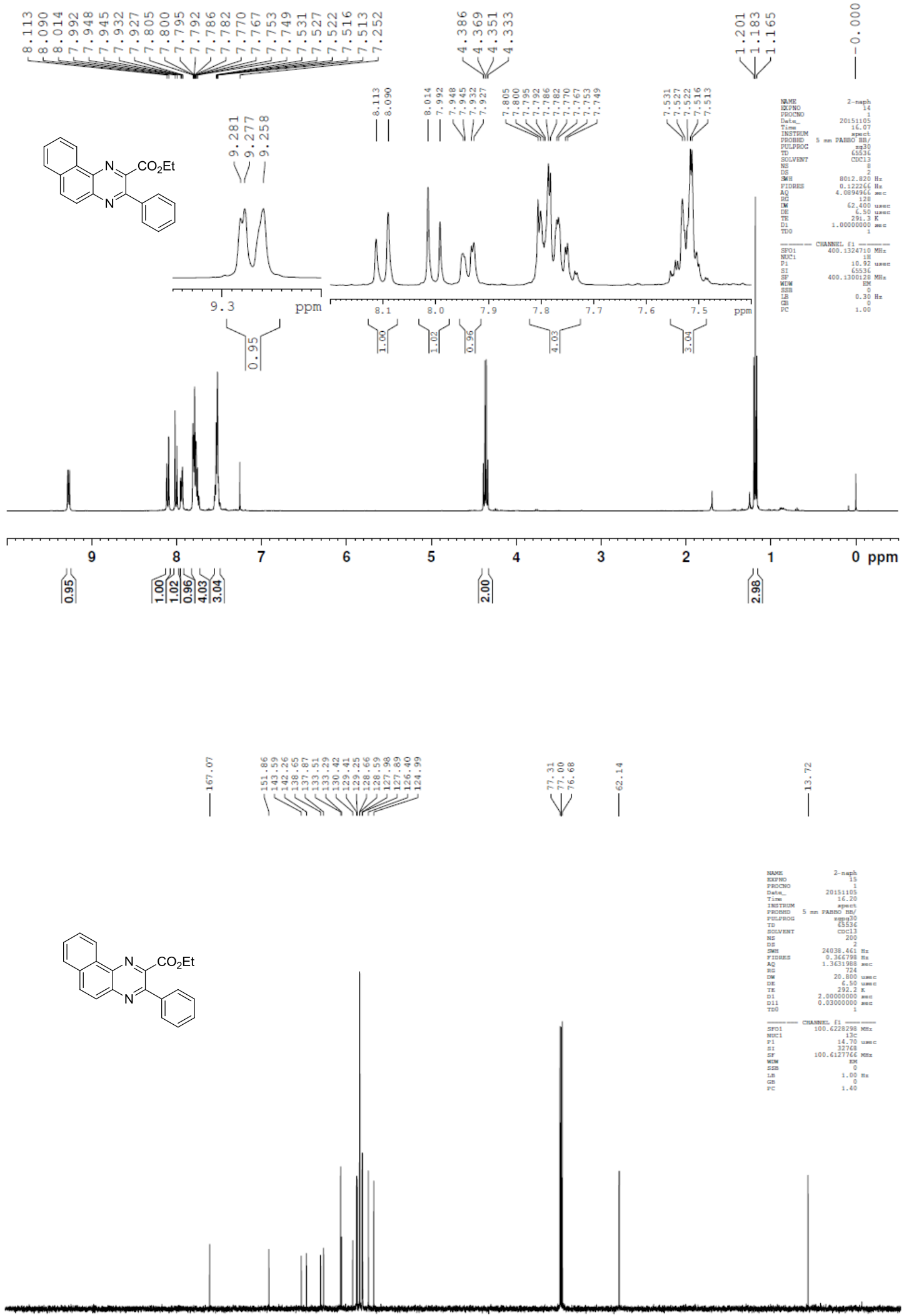

$\begin{array}{lllll}210 & 0\end{array}$ 


\section{$2 \mathbf{t}$}

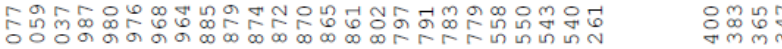

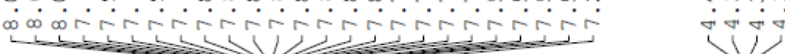

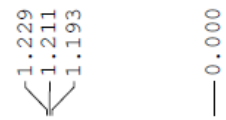
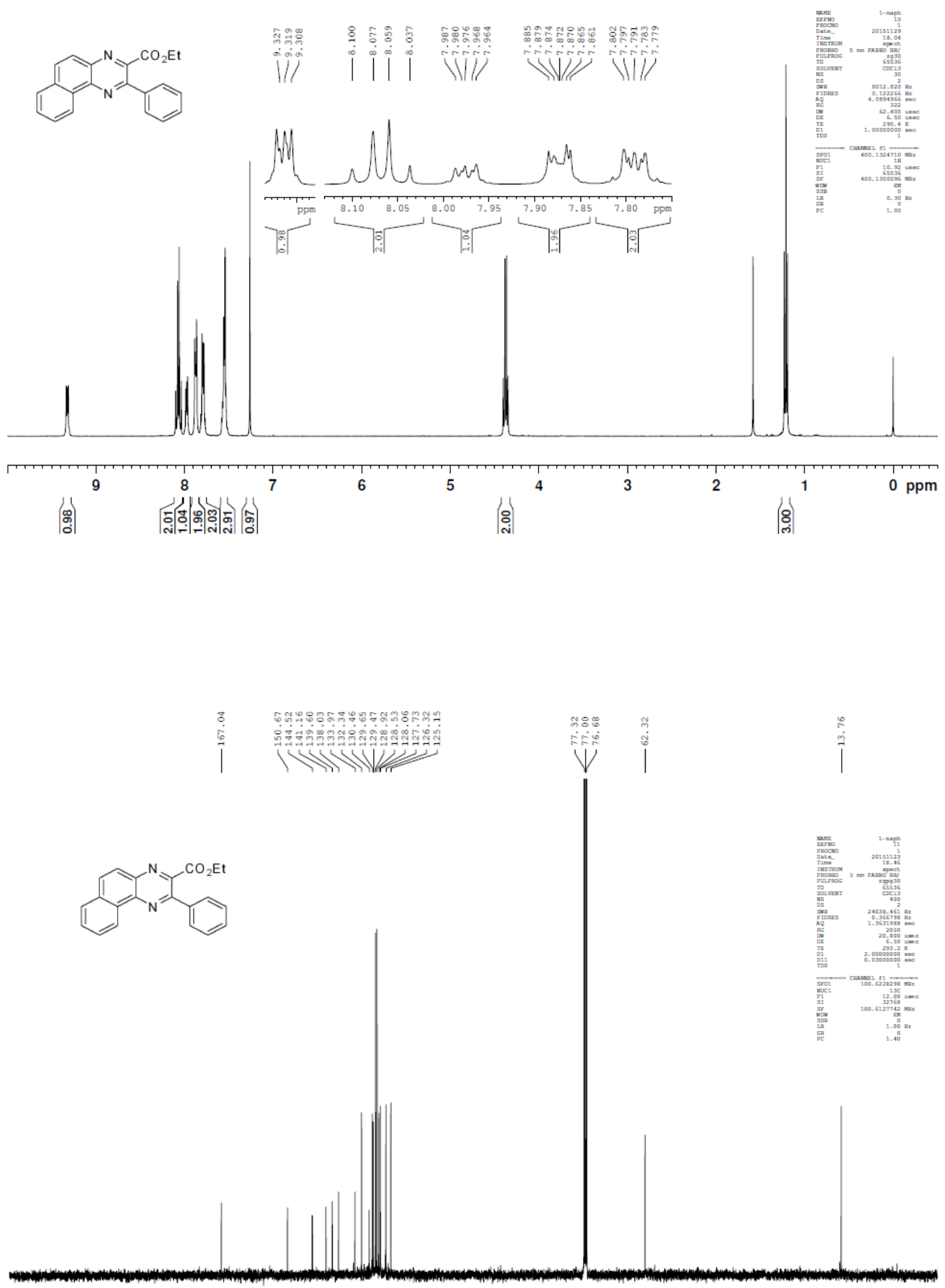

$\begin{array}{llllllllllllllllllllll}210 & 200 & 190 & 180 & 170 & 160 & 150 & 140 & 130 & 120 & 110 & 100 & 90 & 80 & 70 & 60 & 50 & 40 & 30 & 20 & 10 & \mathrm{ppm}\end{array}$ 


\section{2u}

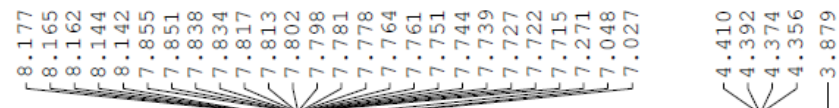

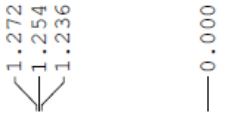

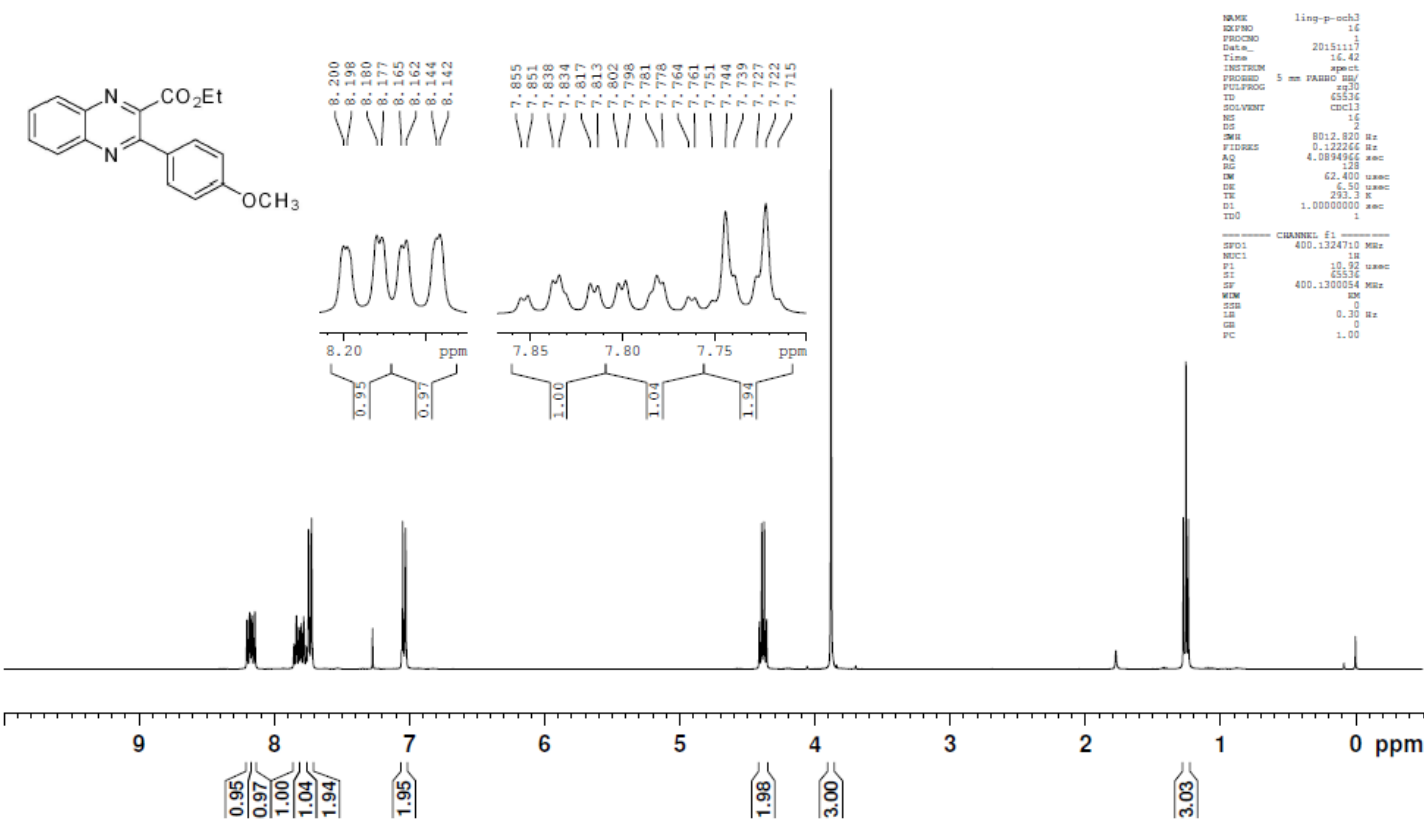

|

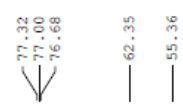

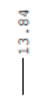
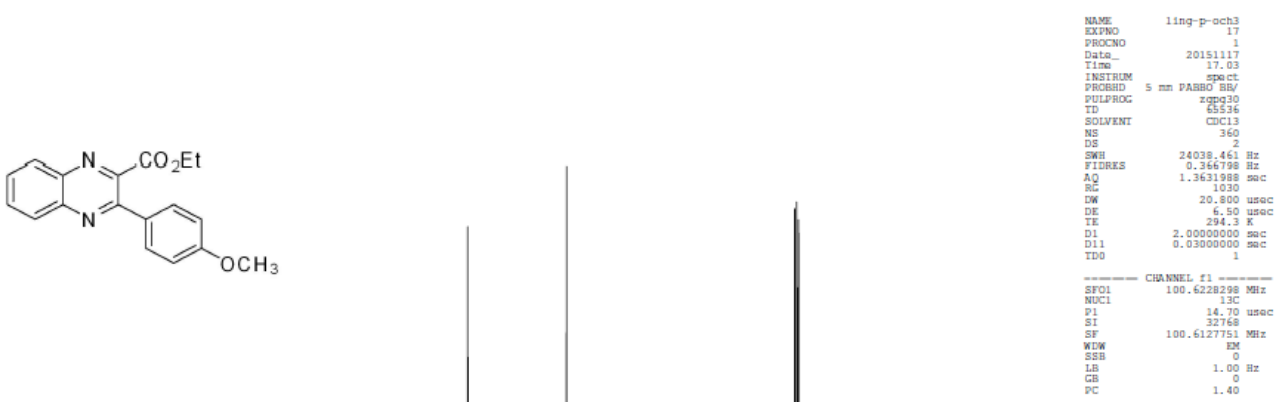

$\begin{array}{lllllllllllllllllllll}210 & 200 & 190 & 180 & 170 & 160 & 150 & 140 & 130 & 120 & 110 & 100 & 90 & 80 & 70 & 60 & 50 & 40 & 30 & 20 & 10\end{array}$ 


\section{2v}

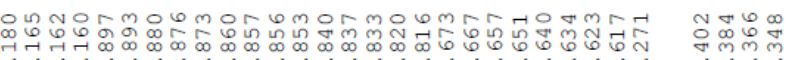

$\infty \infty \infty+\infty$

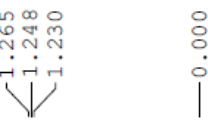
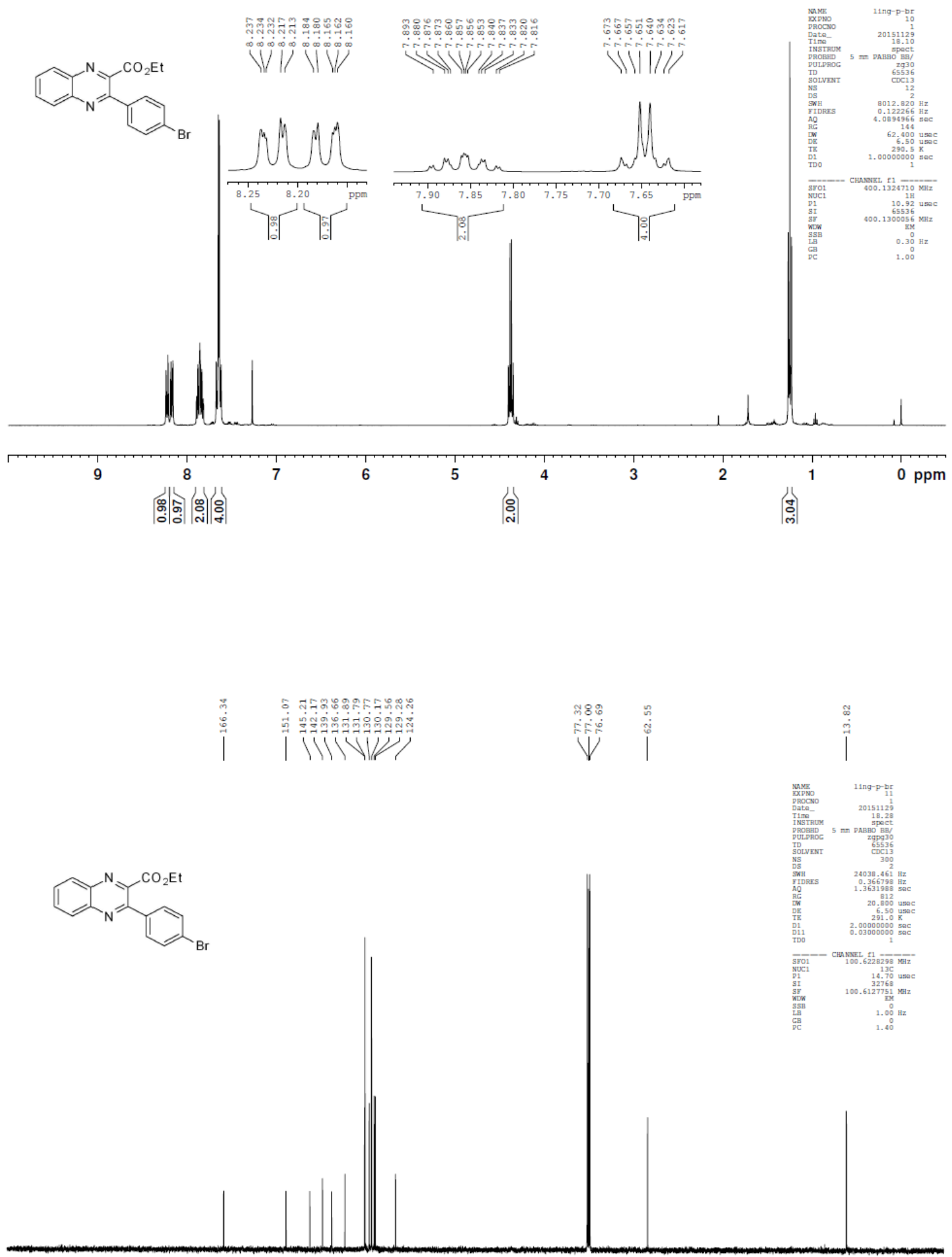

$\begin{array}{llllllllllllllllllllll}210 & 200 & 190 & 180 & 170 & 160 & 150 & 140 & 130 & 120 & 110 & 100 & 90 & 80 & 70 & 60 & 50 & 40 & 30 & 20 & 10 & \mathrm{ppm}\end{array}$ 


\section{2w}

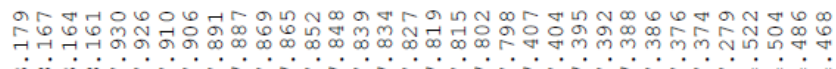

$\dot{0} \dot{\infty} \dot{0} \cdot$

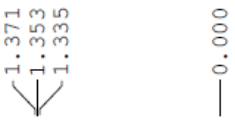
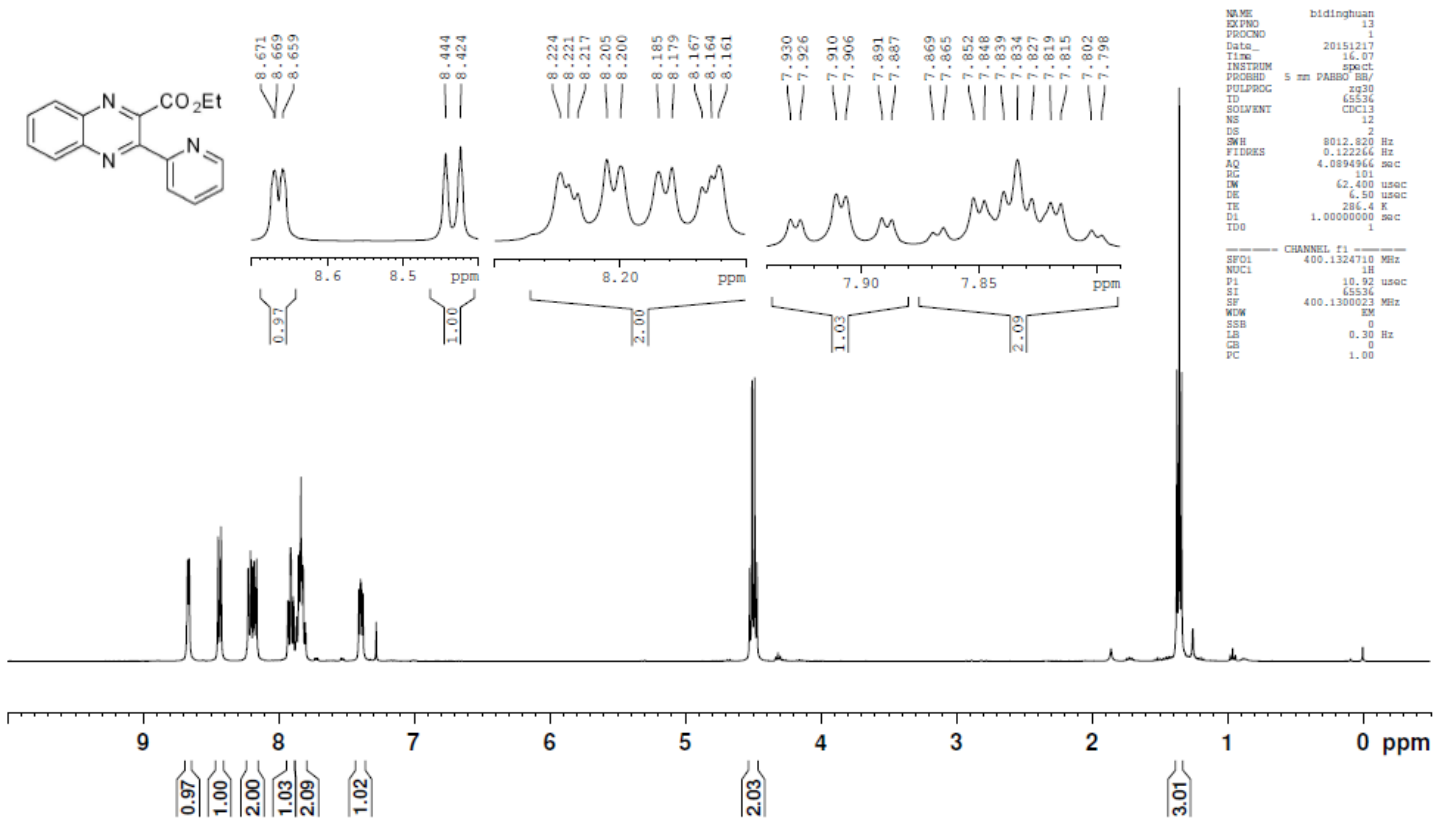

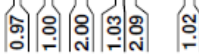

(ึ)

लें
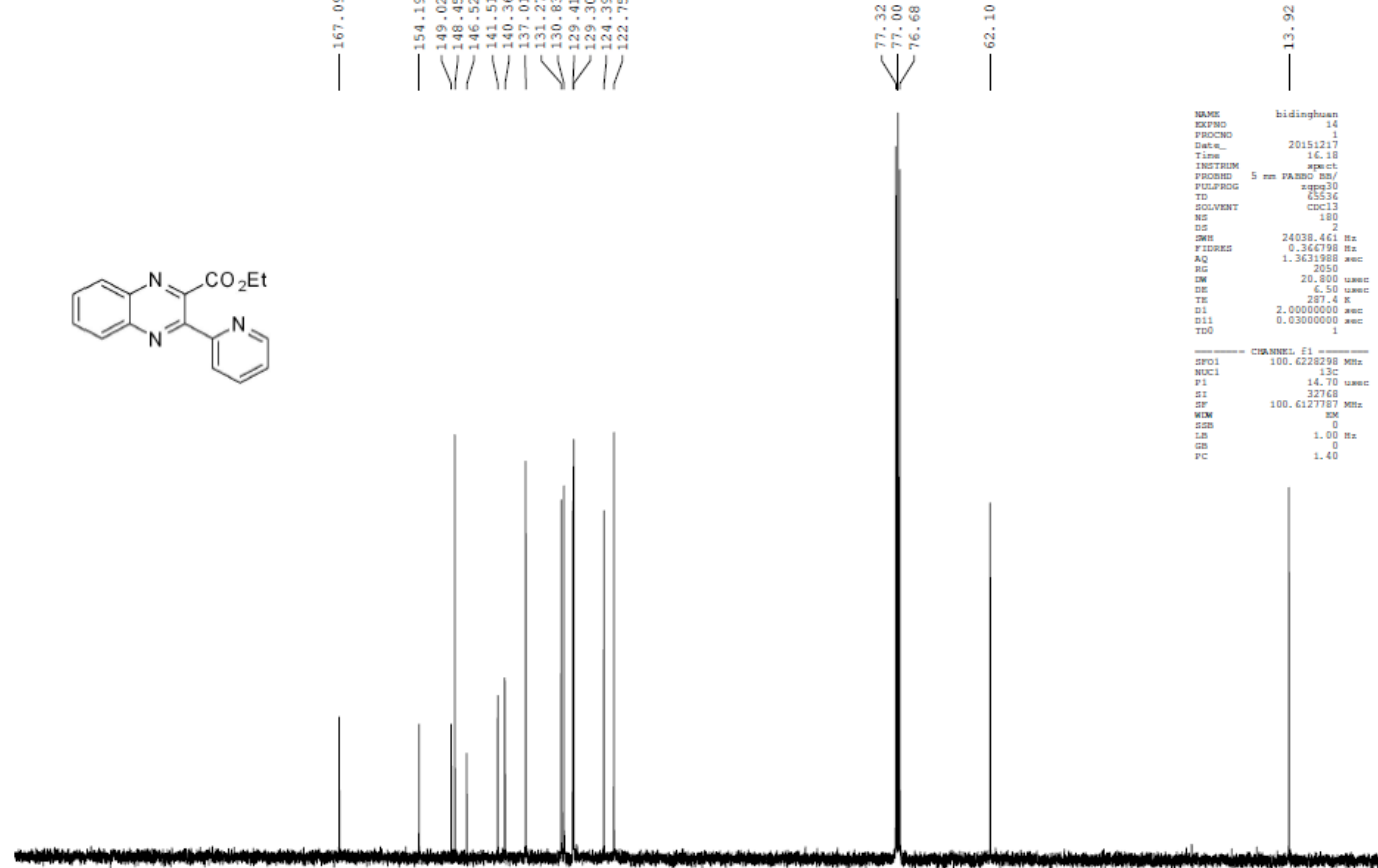

$\begin{array}{llllllllllllllllllllll}210 & 200 & 190 & 180 & 170 & 160 & 150 & 140 & 130 & 120 & 110 & 100 & 90 & 80 & 70 & 60 & 50 & 40 & 30 & 20 & 10 & \mathrm{ppm}\end{array}$ 
2x

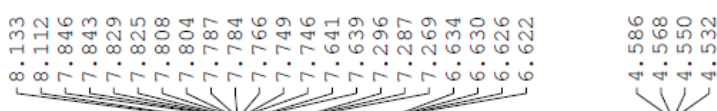

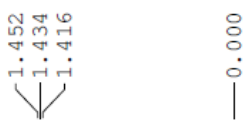

$\overbrace{\mathrm{N}}^{\mathrm{N}}=\mathrm{CO}^{\mathrm{CO}_{2} \mathrm{Et}}$
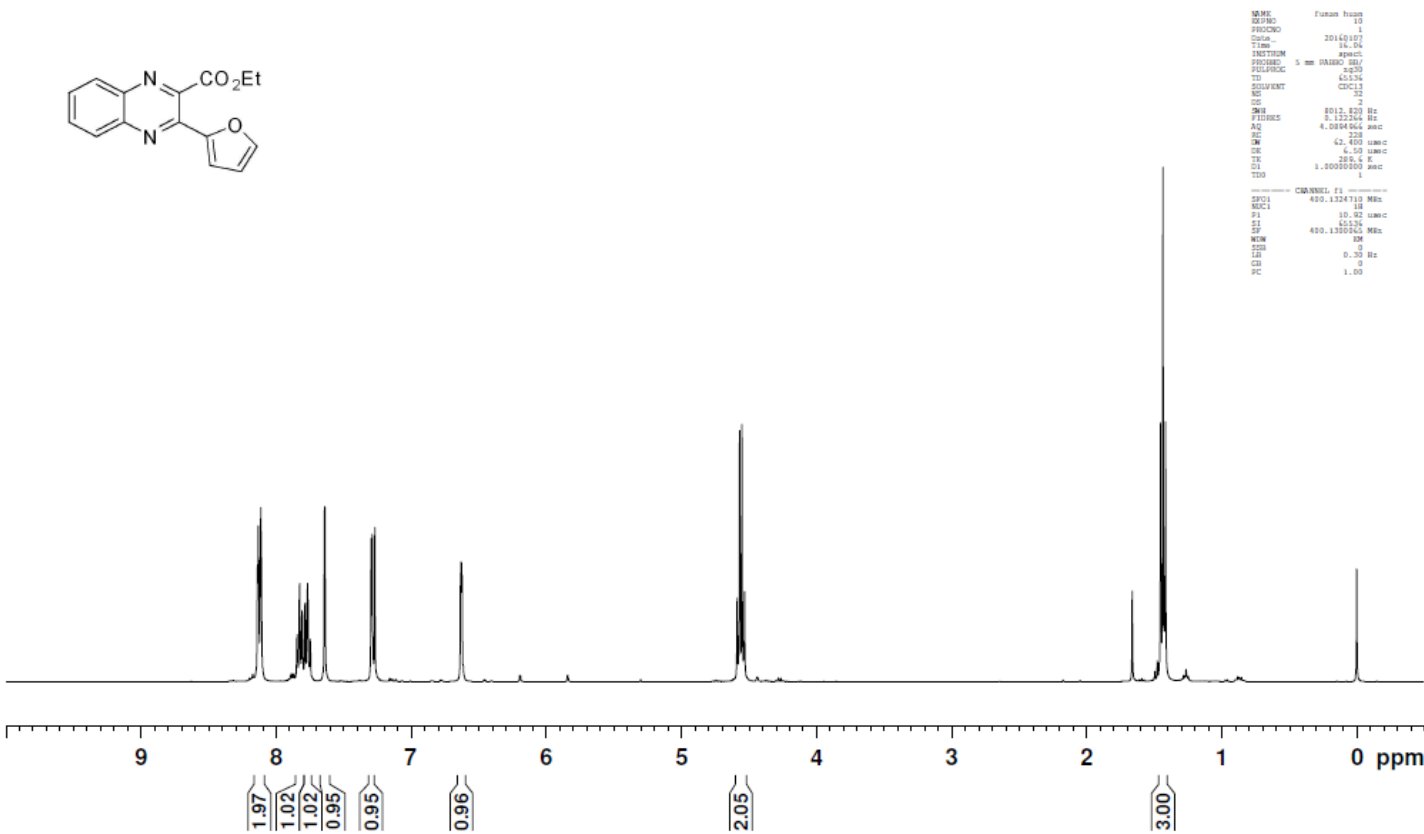

$\overbrace{\mathrm{N}}^{\mathrm{N}} \mathrm{II}_{\mathrm{C}}^{\mathrm{CO}_{2} \mathrm{Et}}$
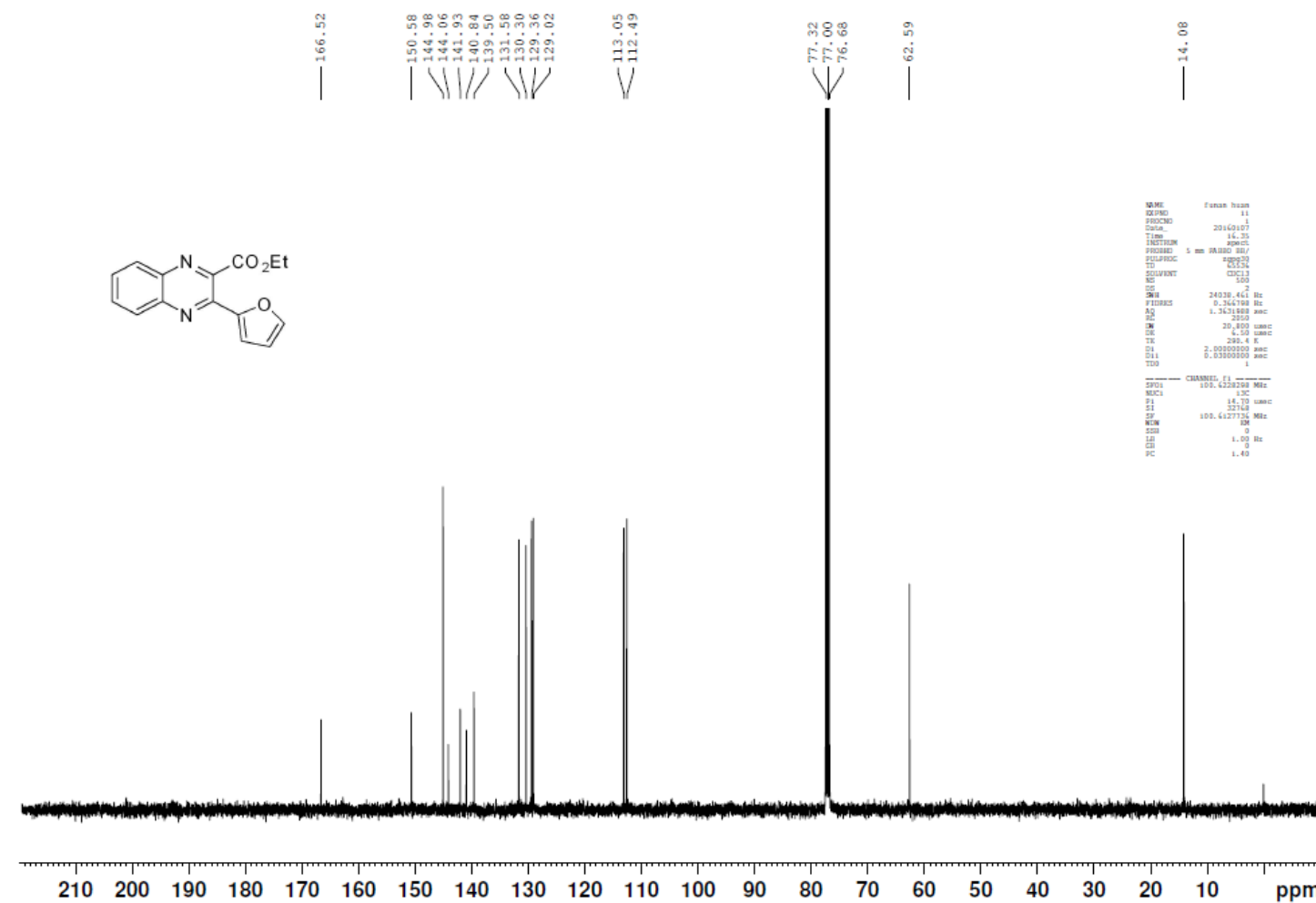
7

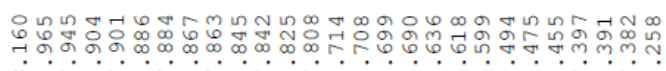

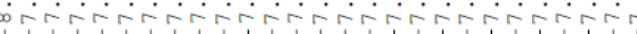
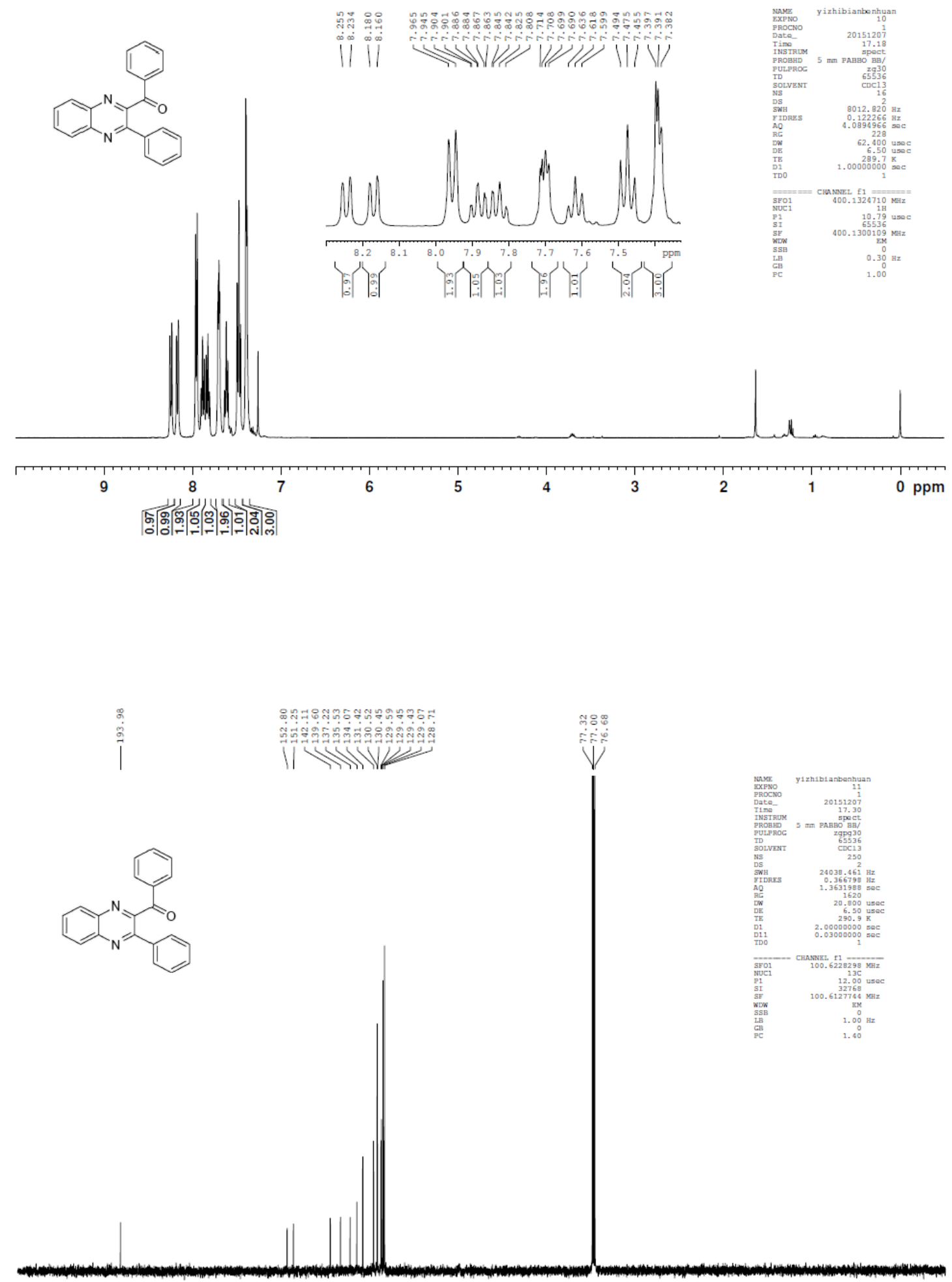

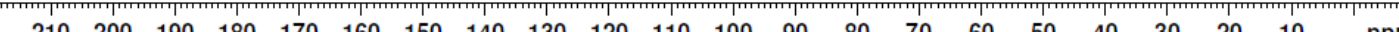


8

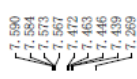

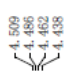

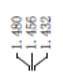

i
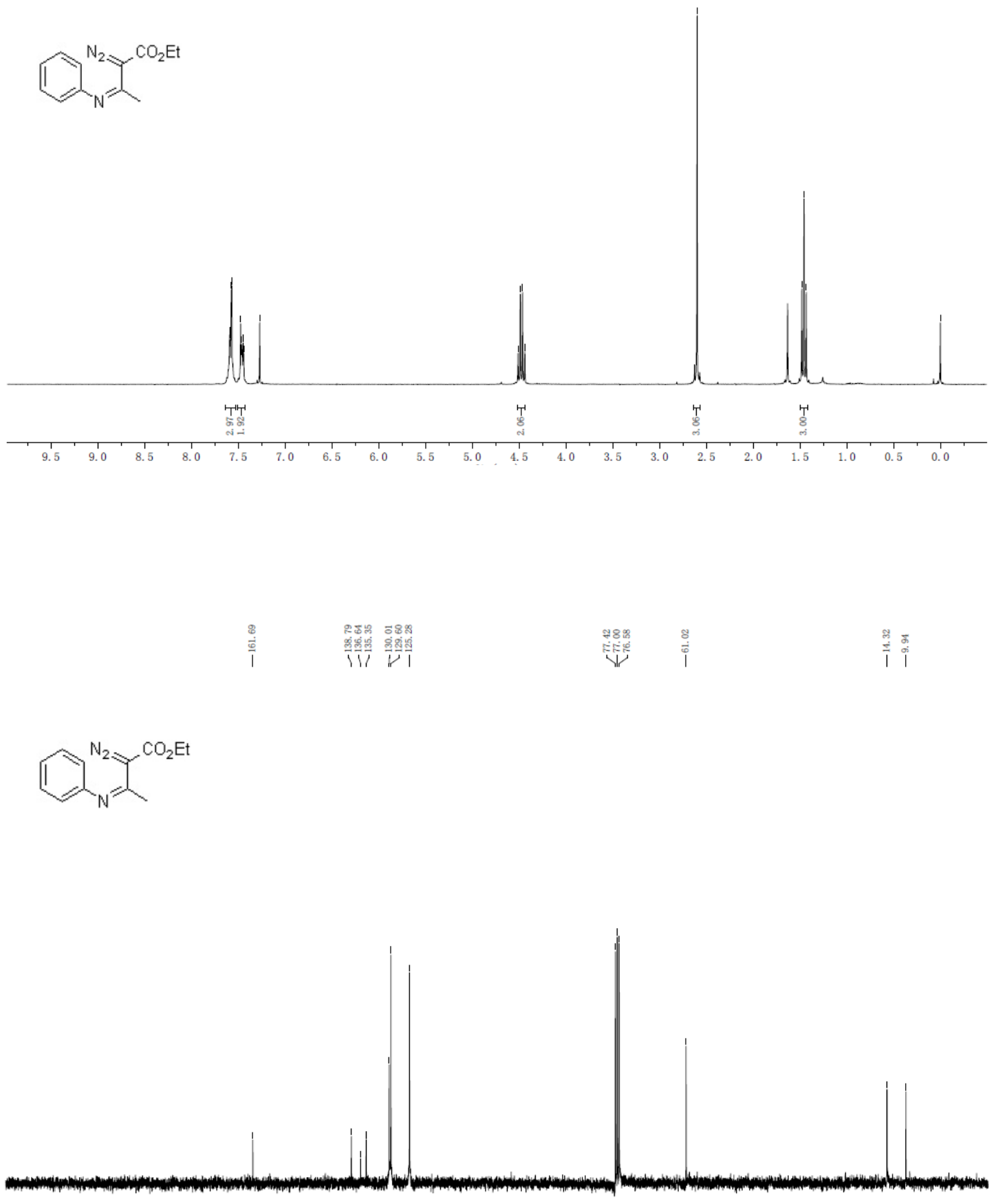

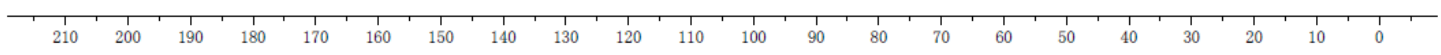

S71 
bobabat in
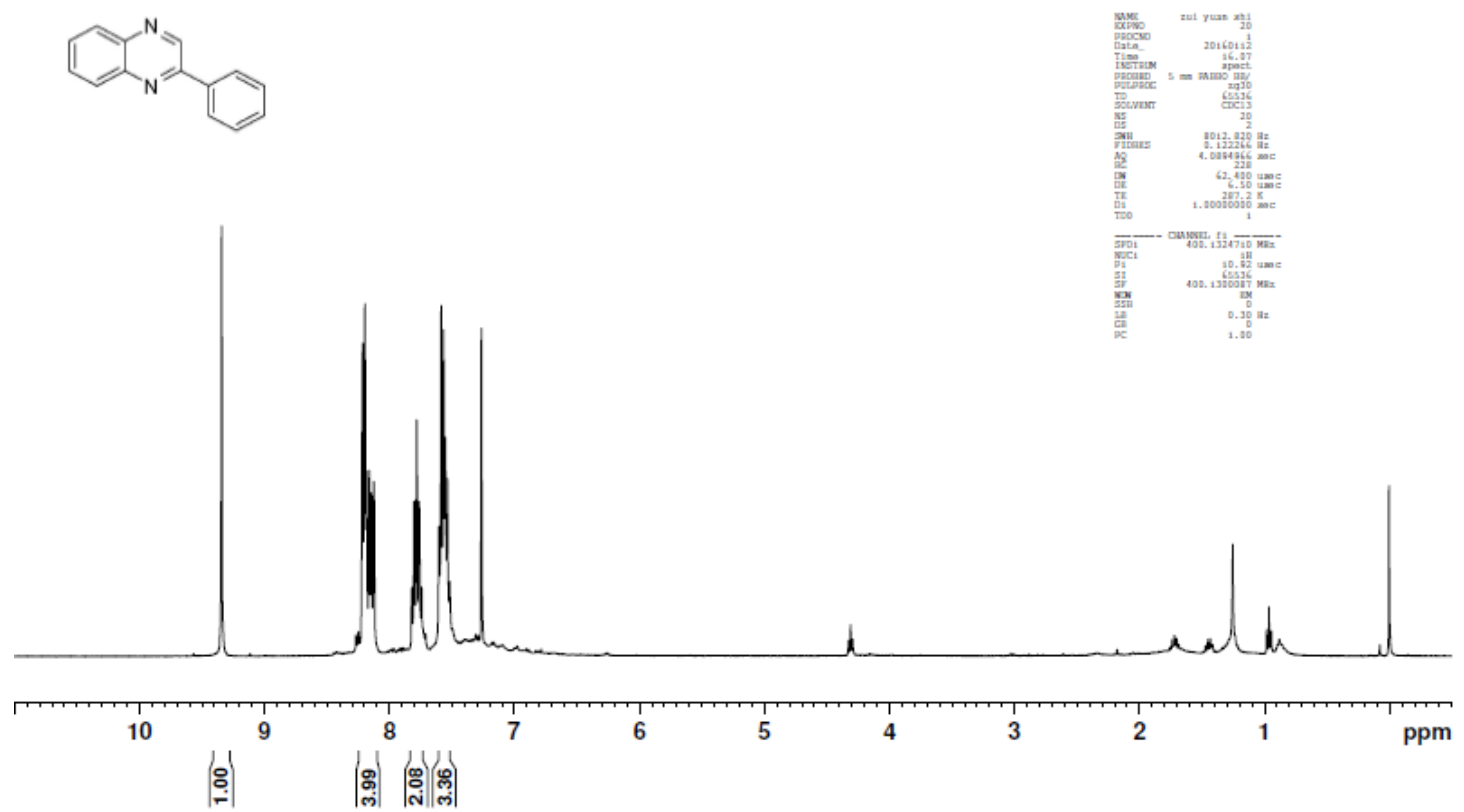

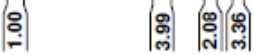

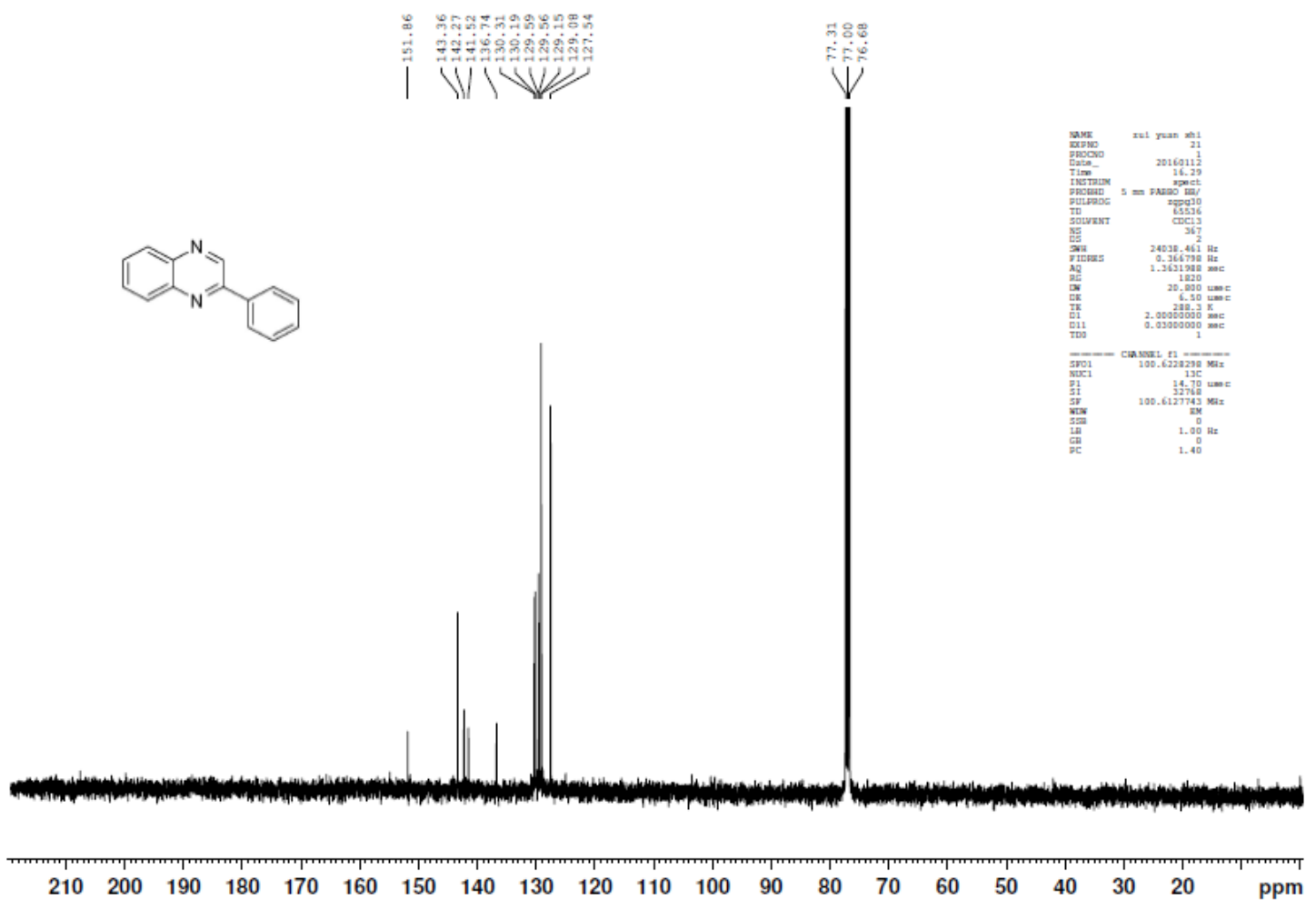



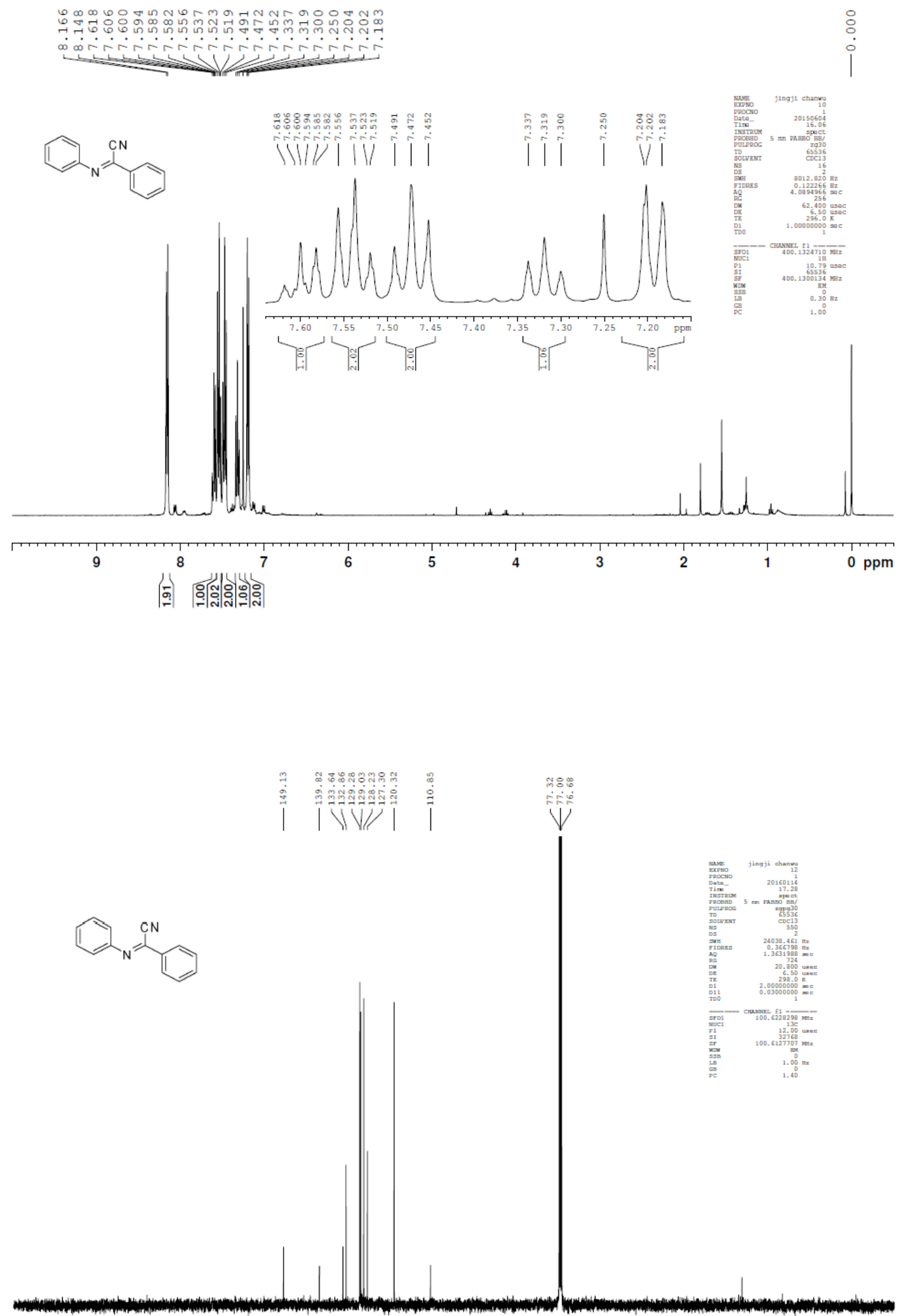

$\begin{array}{llllllllllllllllllllll}210 & 200 & 190 & 180 & 170 & 160 & 150 & 140 & 130 & 120 & 110 & 100 & 90 & 80 & 70 & 60 & 50 & 40 & 30 & 20 & 10 & \mathrm{ppm}\end{array}$ 


\section{Single crystal $X$-ray diffraction data of $2 f$}

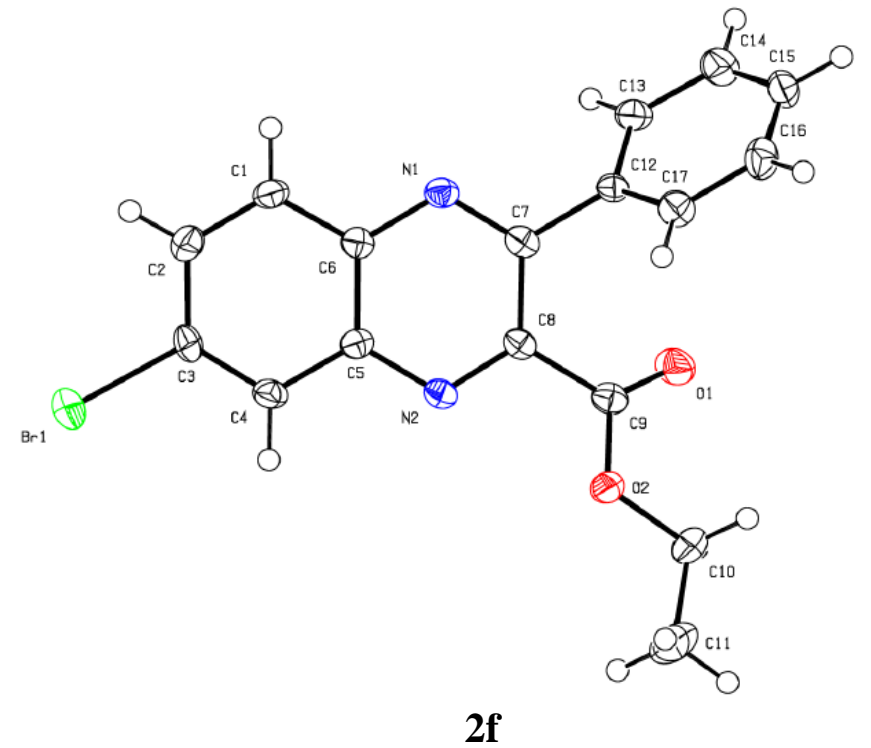

Table 1. Crystal data and structure refinement for $2 \mathbf{f}$ (CCDC 1441580).

Empirical formula

Formula_weight

Crystal system

Space group

Temperature/K

$\mathrm{a} / \AA \AA$

$\mathrm{b} / \AA$

$\mathrm{c} / \AA$

Volume $/ \AA^{3}$

$\beta /{ }^{\circ}$

$\mathrm{Z}$

$\rho \mathrm{mg} / \mathrm{mm}^{3}$

$\mu / \mathrm{mm}^{-1}$

$\mathrm{F}(000)$

Radiation

$2 \theta$ range for data collection

Index ranges

Reflections collected

Independent reflections

Data/restraints/parameters

Goodness-of-fit on $\mathrm{F}^{2}$

Final $\mathrm{R}$ indexes $[\mathrm{I}>=2 \sigma(\mathrm{I})]$

Final $\mathrm{R}$ indexes [all data]

Largest diff. peak/hole / e $\AA^{-3}$
$\mathrm{C}_{17} \mathrm{H}_{13} \mathrm{BrN}_{2} \mathrm{O}_{2}$

357.20

monoclinic

$\mathrm{P} 2{ }_{1} / \mathrm{c}$

294.06(10)

$4.4677(2)$

16.8234(8)

$20.1968(11)$

1516.53(13)

92.566(5)

4

1.565

2.719

720.0

$\operatorname{MoK} \alpha(\lambda=0.71073)$

7.54 to $52.04^{\circ}$

$-5 \leq \mathrm{h} \leq 5,-20 \leq \mathrm{k} \leq 9,-24 \leq 1 \leq 24$

5862

$2999\left[\mathrm{R}_{\text {int }}=0.0381, \mathrm{R}_{\text {sigma }}=0.0673\right]$

2999/0/200

1.045

$\mathrm{R}_{1}=0.0432, \mathrm{wR}_{2}=0.0741$

$\mathrm{R}_{1}=0.0680, \mathrm{wR}_{2}=0.0838$

$0.40 /-0.33$ 